\title{
Assessment and Improvement of the Capabilities of a Window Correlator to Model GPS Multipath Phase Errors
}

David F. Bétaille

Thesis submitted for the degree of

Doctor of Philosophy

of the University of London

Department of Geomatic Engineering

University College London

2004 
Theory guides. Experiment decides.

DonaldE. Simank (1936-) US physidst, educator, humorist. 


\begin{abstract}
This thesis is concerned with the modelling of Global Positioning System (GPS) phase multipath. GPS is increasingly used for very high precision (centimetre level) engineering surveying applications such as setting out on construction sites and the control of major civil engineering plant (e.g. bulldozers, graders and pavement layers). In such applications the phase of the carrier signal is the basic observable and the dominant error source is multipath (electromagnetic reflections of the carrier waves from surfaces in the surroundings of the antennas). The work contained in the thesis has been carried out in collaboration with the LCPC (Laboratoire Central des Ponts et Chaussées) in Nantes, France, which made available its test facility and Leica Geosystems, Heerbrugg, Switzerland, a GPS manufacturer, which funded the work and which made available modified equipment for testing.
\end{abstract}

The specific subject of the research is the assessment and improvement of the capabilities of a Phase Multipath Mitigation Window correlator (PMMW) to model GPS multipath phase errors. The phase window correlator is a new sampling technique dedicated to the estimation of multipath errors in phase measurements. The thesis contains background material on GPS multipath mitigation and on several existing patents related to the PMMW technique. The main contribution of the work relates to:

- The rigorous mathematical modelling of multipath, starting from the physics of the phenomenon, right through to the phase measurement process itself, particularly that based on the PMMW correlator.

- The design of a general testing methodology in a controlled environment to assess the efficacy of multipath mitigation techniques. 
- The carrying out of full-scale experiments at the LCPC in both static and kinematic modes and the assessment of the performances and limitations of the PMMW correlator.

- Initial investigations into the design of a new real-time correction strategy for phase multipath phase errors based on a combination of multipath observables including the signal-to-noise ratio, the output from the PMMW correlator and an estimation of the code multipath errors from dual frequency phase data. A key feature of the new strategy is its ability to overcome the major limitation of the PMMW technique, i.e. its insensitivity to multipath caused by very close reflectors. It enables the multipath corrupted phase measurements to be improved by $10 \%$ in average in this case, whereas the PMMW corrections on their own had almost no effect. 


\section{ACKNOWLEGMENTS}

This work was funded by Leica Geosystems and the LCPC.

I would like to thank my supervisor, Professor Paul Cross, for giving me the opportunity to undertake this program of research, and for his welcome in the Geomatic Engineering Department at UCL and his continuous support. Thanks also to François Peyret for supporting this project at the LCPC.

I would like to thank generally all the staff of the GE Department whom I met during my twenty-two 3-days round-trips to London, and particularly the GPS, geodesy and navigation group. I did appreciate their welcome.

Thanks also to Hans-Juergen Euler (Leica Geosystems, Heerbrugg) and Jon Maenpa (Leica Geosystems, Torrance) for sharing their extensive GPS knowledge and experience, and for their guidance in determining the directions in which this research has moved. Thanks are also due a number of their colleagues and particularly GPS senior consultants Thomas A. Stansell, Jerry E. Knight, Rich G. Keegan, Ron R. Hatch and Charles R. Cahn, some of them I met in Leica or in ION congresses, and whom I am indebted as the inventors of a few techniques that I used in this work.

I also would like to thanks for their technical support Charles Lemaire and Jean-Marie Prual and all the Robotics and Localisation group at LCPC. During the campaigns of tests, we were also helped by Nicolas de Moegen and Emmanuel Roy of Leica France, who provided the receivers, and whom I here thanks again.

Joel Barnes, Stéphane Durand, Kevin Sheridan and Marek Ziebart were of great help in solving problems in space geodesy, so thanks a lot! Thanks also to Mike Braasch and, last but not least, to Vincent Baltazart, both of very good advice, particularly in physics. 
To Sylvie (many many thanks to her!), and our children... 


\title{
CONTENTS
}

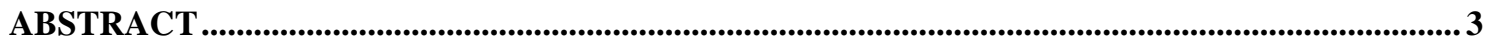

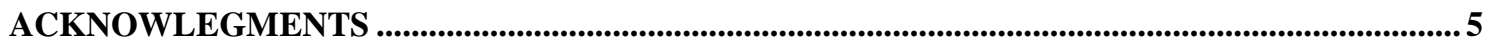

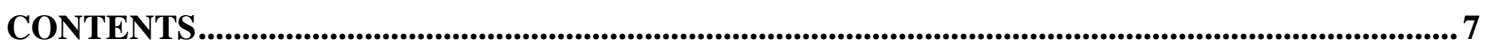

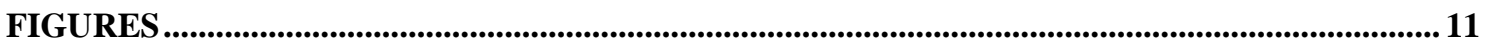

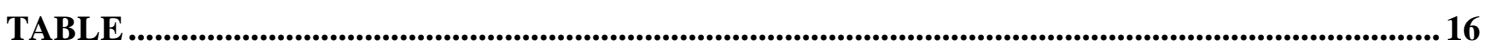

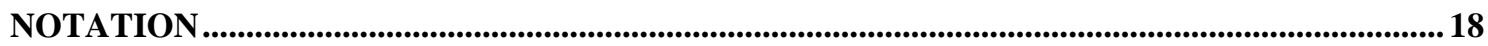

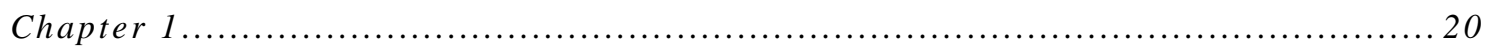

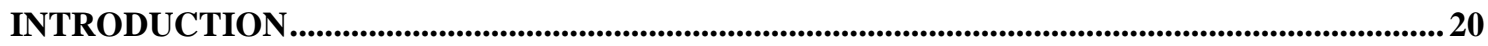

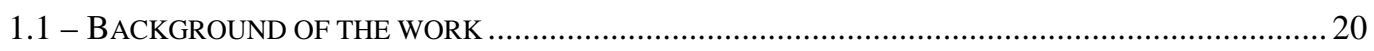

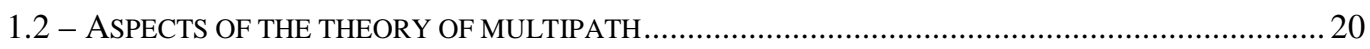

1.2.1 - Origin of multipath and local model of propagation................................................... 21

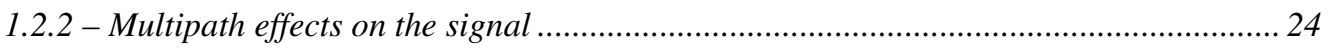

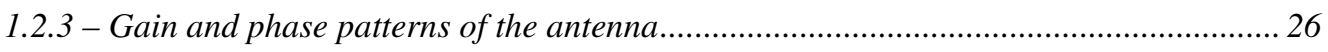

1.2.4 - Representation of the GPS received signal in the receiver ..........................................2 27

1.2.5 - Overall consequences of multipath in the range measurements................................. 28

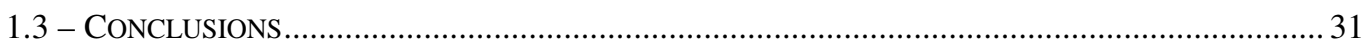

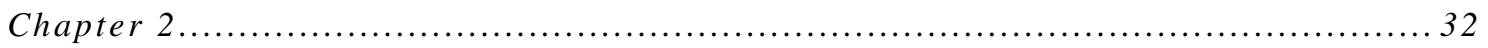

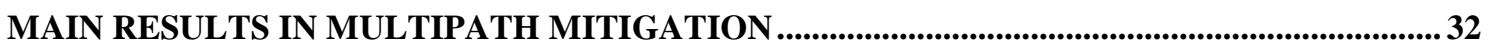

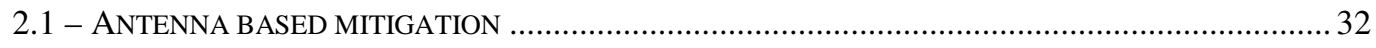

2.1.1 - Specific features of standard GPS antennas related to multipath ................................. 32

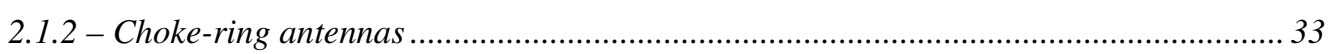

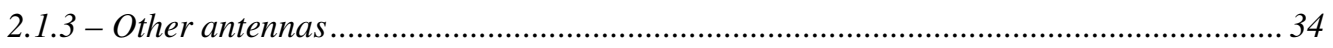

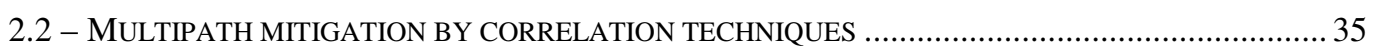

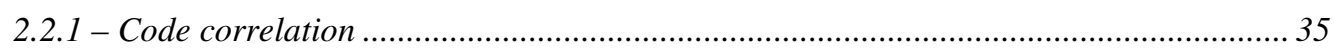

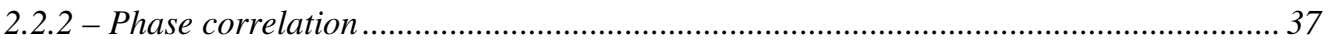

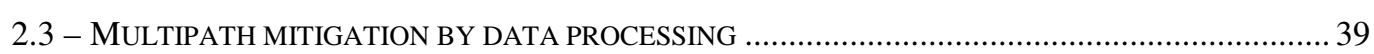


2.3.1 - Functional modelling

2.3.1.1 - L1 and L2 phase combination to estimate multipath on code data ....................................... 39

2.3.1.2 - L1 and L2 ionospheric differential delay to estimate multipath on phase data..................... 41

2.3.1.3 - Using the signal-to-noise ratio (SNR) as an observable of the carrier phase multipath ....... 44

2.3.1.4. - Using multiple antennas to remove multipath .................................................................... 46

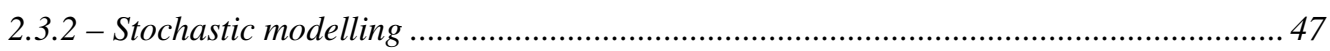

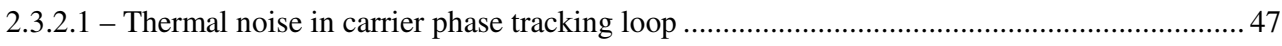

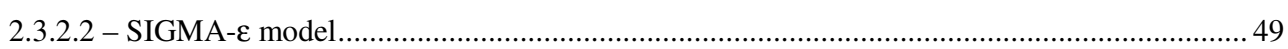

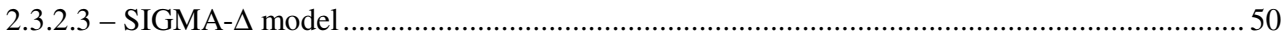

2.3.2.4 - Extended weight model for GPS phase observations ........................................................ 51

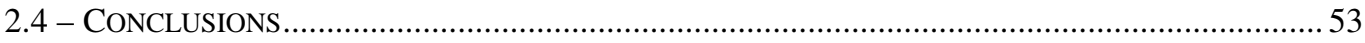

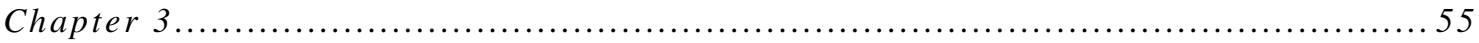

GPS SIGNAL PHASE AND CODE TRACKING IN A STANDARD RECEIVER ..........................55

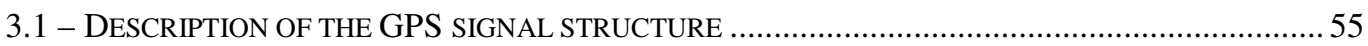

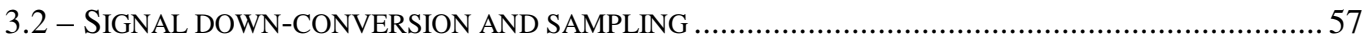

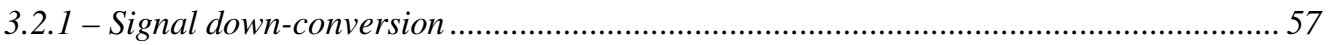

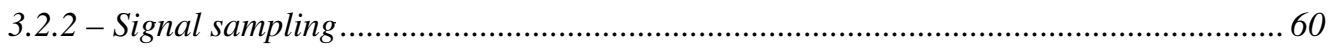

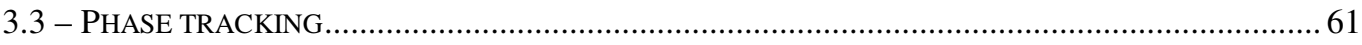

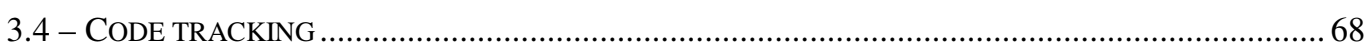

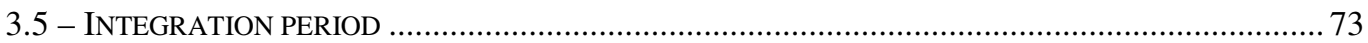

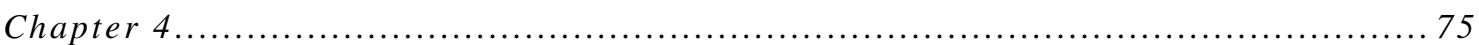

PHASE AND CODE TRACKING IN THE PRESENCE OF MULTIPATH ................................ 75

4.1 - PHASE TRACKING IN THE PRESENCE OF MULTIPATH ...................................................... 75

4.2 - CODE TRACKING IN THE PRESENCE OF MULTIPATH ........................................................ 80

4.2 .1 - Case of the "wide" correlator code tracking ........................................................... 80

4.2.2 - Case of the "narrow" correlator code tracking........................................................ 87

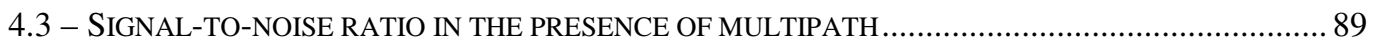

4.3.1 - Definition of the carrier-to-noise and signal-to-noise ratio ...................................... 89

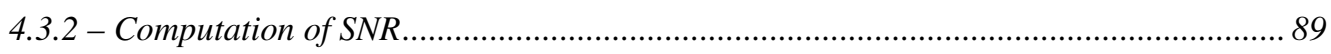

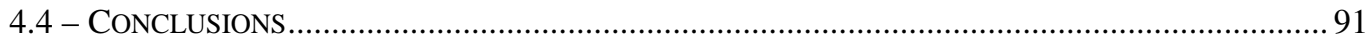

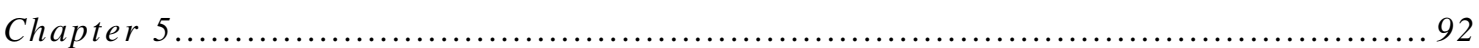

PHASE AND CODE MULTIPATH MITIGATION WINDOW TECHNIQUES .............................92

5.1 - MMW PROCESS APPLIED ON CODE TRACKING ........................................................... 93 
5.1.1 - Standard "wide" and "narrow" code correlators. .93

5.1 .2 - Multipath mitigation by "reference waveform" code correlators.............................. 94

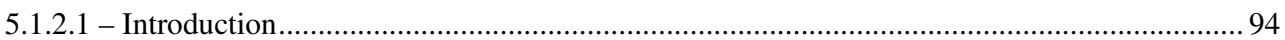

5.1.2.2 - Generation of the Multipath Mitigation Window .............................................................. 95

5.1.2.3 - Correlation of the signal with the Multipath Mitigation Window (type A) .......................... 95

5.1.2.4 - Correlation of the signal with the Multipath Mitigation Window (type B) .......................... 97

5.1.2.5 - Asymmetric code Multipath Mitigation Window ............................................................. 99

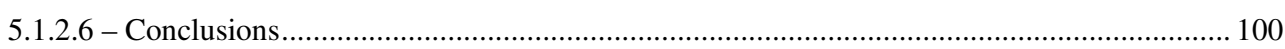

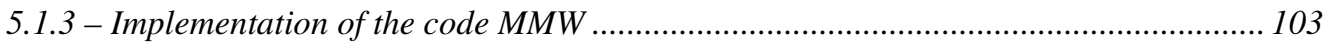

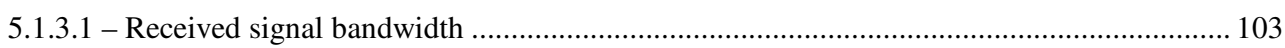

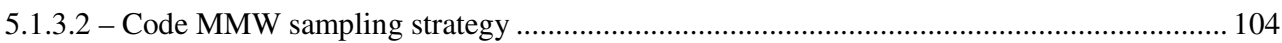

5.1.4 - Conclusions relating to the Code Multipath Mitigation Window process................... 105

5.2 - MMW PROCESS APPLIED TO PHASE TRACKING ............................................................. 106

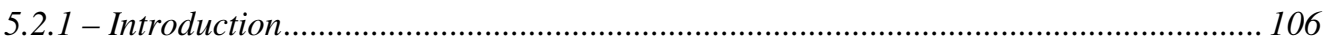

5.2 .2 - Recall of the meaning of the phasor diagram in the presence of multipath ............... 106

5.2.3 - The phase MMW correlator .................................................................................. 109

5.2 .4 - Implementation of the phase MMW..................................................................... 110

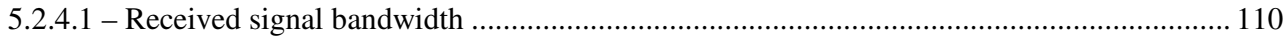

5.2.4.2 - Asymmetric phase Multipath Mitigation Window .......................................................... 111

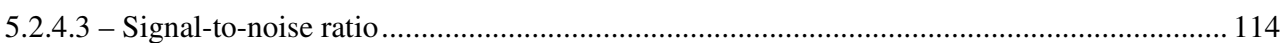

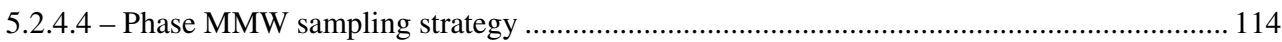

5.2.5 - Conclusions about Phase Multipath Mitigation Window process.............................. 115

5.3 - OVERALL CONCLUSIONS ABOUT MMW ......................................................................... 115

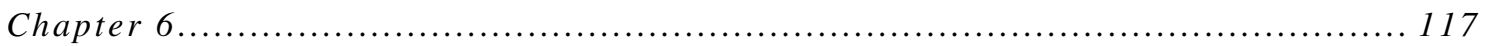

TESTING THE PHASE MULTIPATH MITIGATION WINDOW .................................................... 117

6.1 - THE GPS EQUIPMENT USED AND THE SESSYL FACILITY .............................................. 117

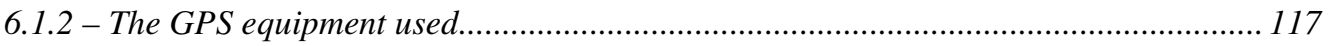

6.1 .2 - SESSYL and the multipath testing equipment ................................................... 118

6.2 - PREDICTION OF MULTIPATH FROM THE PANEL .............................................................. 122

6.2.1 - Definition of used reference frames .................................................................. 122

6.2.2 - Computation of the multipath window time zones................................................... 123

6.2.3 - Prediction of the multipath phase error ..................................................................... 129

6.2 .4 - Tuning the parameters $\alpha$ and $\Theta$ of the model .................................................... 131

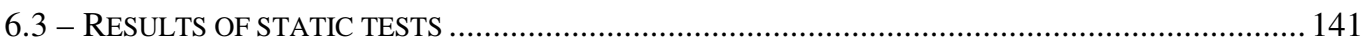

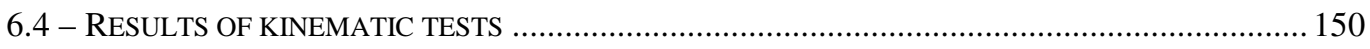

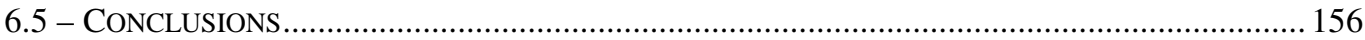


Chapter 7 .

IMPROVING THE PHASE MMW BY USING MULTIPATH FUNCTIONAL MODELING...... 157

7.1 - APPROXIMATION OF THE MULTIPATH MODELLING THEORY ……............................................ 157

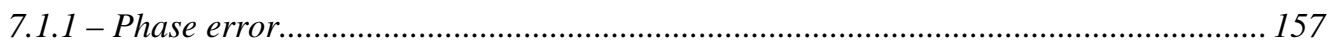

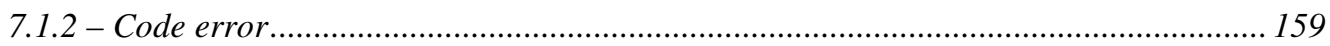

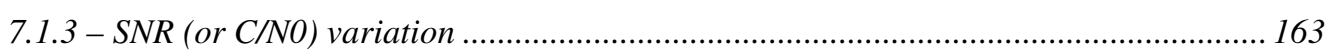

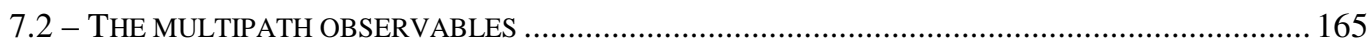

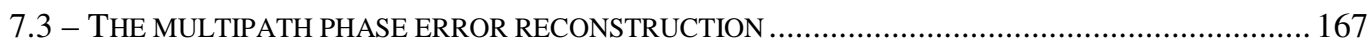

7.3.1 - SNR (or C/NO) based multipath phase error reconstruction....................................... 167

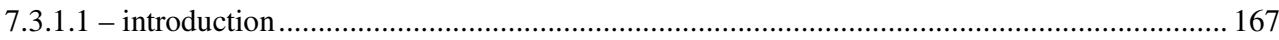

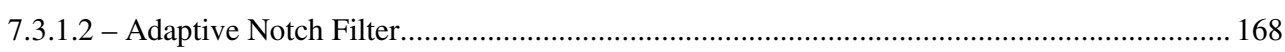

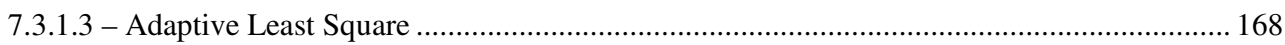

7.3.1.4 - Application of the combined ANF\&ALS process to SNR ................................................ 169

7.3.2 - SNR (or C/NO) and phase MMW based reconstruction ............................................. 171

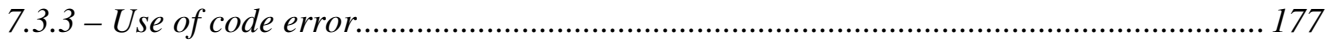

7.3.4 - Fusion of the reconstruction processes ................................................................ 180

7.4 - GENERALISATION OF THE APPLICATION OF THE ALGORITHM ........................................... 183

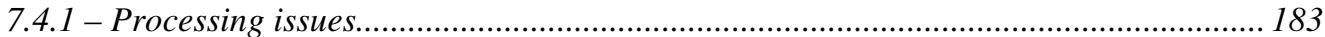

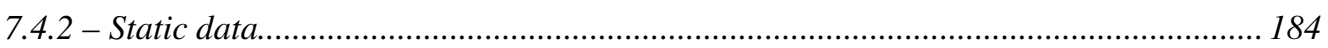

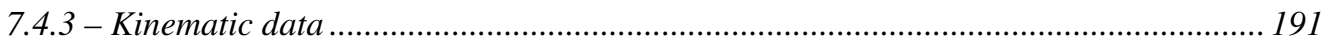

7.5 - CONCLUSIONS ABOUT THE RECONSTRUCTION PROCESSES ................................................ 197

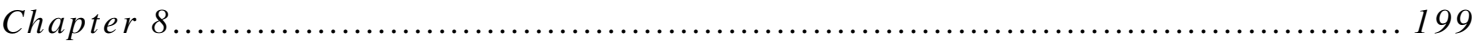

CONCLUSIONS AND SUGGESTIONS FOR FURTHER WORK........................................................199

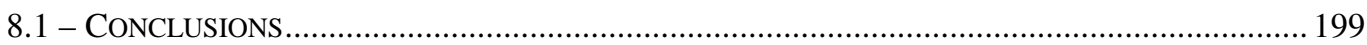

8.2 - SUGGESTIONS FOR FURTHER WORK ............................................................................... 202

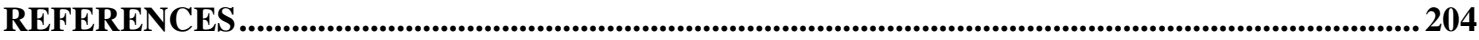

APPENDIX 1: OPTIMISATION OF THE SHAPE OF THE PHASE MMW ...............................210

APPENDIX 2: ELECTROMAGNETIC MODELLING ..................................................................... 212

APPENDIX 3: AERIAL PHOTO OF THE SESSYL SITE ................................................................. 221

APPENDIX 4: RESULTS OF THE STATIC TESTS WITH CHOKE-RING ANTENNAS .......... 222

APPENDIX 5: STUDY OF THE BACKGROUND MULTIPATH FROM SESSYL ...................... 226 


\section{FIGURES}

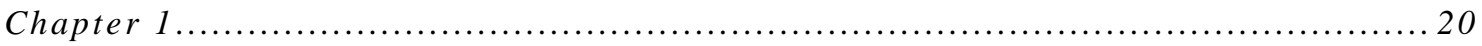

Figure 1.1: direct path and reflected paths ...................................................................... 21

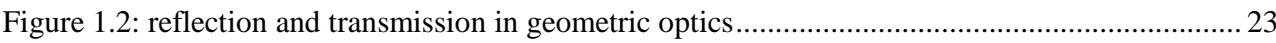

Figure 1.3: static survey showing both diffraction and reflection .................................................. 30

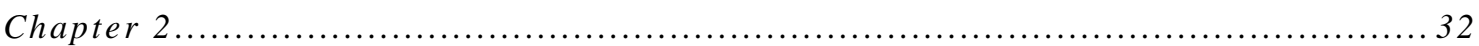

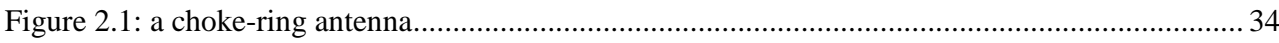

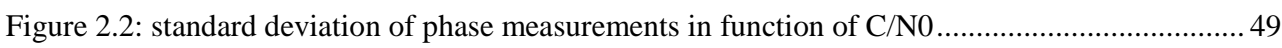

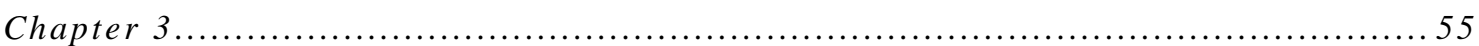

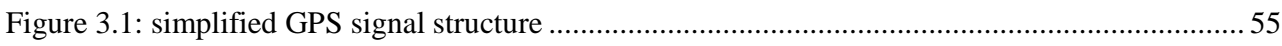

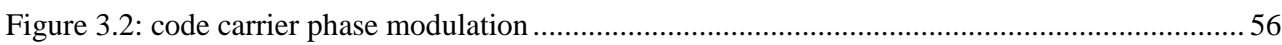

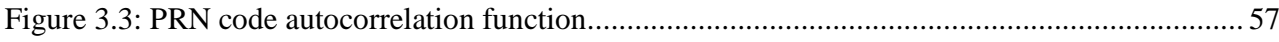

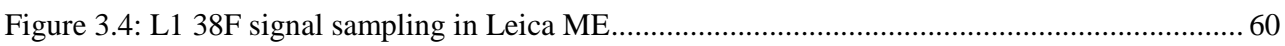

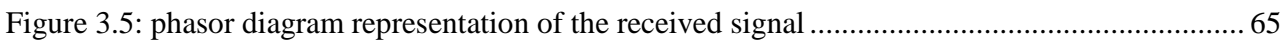

Figure 3.6: received Doppler frequency in a static application ........................................................ 66

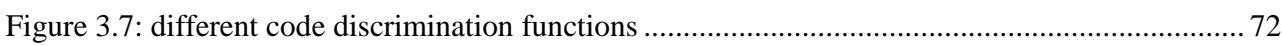

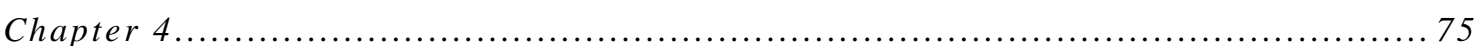

Figure 4.1: phasor diagram representation of the composed (direct + reflected) signal ..................... 79

Figure 4.2: maximum phase tracking errors in the presence of multipath.......................................... 80

Figure 4.3: deviation of the "non coherent dot-product power" DF in the presence of multipath....... 84

Figure 4.4: deviation of the "coherent early-late envelope" DF in the presence of multipath............. 85

Figure 4.5: maximum C/A and P codes tracking errors in the presence of multipath (source: Leica) 86

Figure 4.6: wide and narrow "early-late envelope" discrimination functions

Figure 4.7: maximum C/A-code tracking errors for wide and narrow "early-late envelop" discrimination function (source: Leica)

Chapter 5 .

Figure 5.1: maximum C/A and P codes tracking errors for wide and narrow "early-late envelop" discrimination function (source: Leica)

Figure 5.2: generation of the reference waveform in a MMW correlator ......................................... 95

Figure 5.3: MMW type A correlation process .............................................................................. 96 
Figure 5.4: MMW type A discrimination function

Figure 5.5: maximum C/A-code tracking errors for MMW type A discrimination function (Leica) .. 97

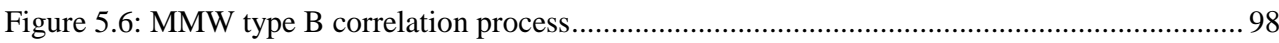

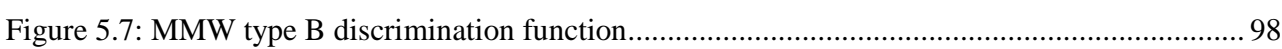

Figure 5.8: maximum C/A-code tracking errors for MMW type B discrimination function (Leica) .. 99

Figure 5.9: asymmetric MMW type B correlation process .......................................................... 100

Figure 5.10: asymmetric MMW type B discrimination function ................................................... 100

Figure 5.11: incorrect asymmetric MMW correlation process (the overall surface of the second half part of the MMW is not zero).

Figure 5.12: incorrect asymmetric MMW discrimination function (the overall surface of the second half part of the MMW is not zero)

Figure 5.13: maximum C/A-code tracking errors for the different correlators listed above ............. 103

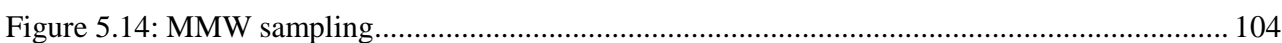

Figure 5.15: direct and multipath signals entering the standard phase loop ..................................... 107

Figure 5.16: phasor diagram of direct and multipath signals corresponding to the standard phase loop correlator

Figure 5.17: direct and multipath signals entering the MMW phase loop. 109

Figure 5.18: phasor diagram of direct and multipath signals corresponding to the MMW phase loop correlator

Figure 5.19: one chip delayed multipath signal

Figure 5.20: phasor diagram of direct and multipath signals corresponding to the MMW phase loop correlator, in case of a preceding chip with the same polarity

Figure 5.21: phasor diagram of direct and multipath signals corresponding to the MMW phase loop correlator, in case of a preceding chip with the opposite polarity 112

Figure 5.22: MMWs "symmetric at transitions only" and "asymmetric at every code clock".

Figure 6.1: SESSYL carriage on its track

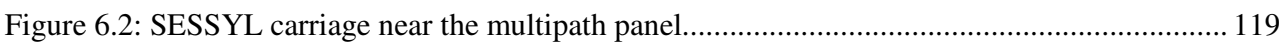

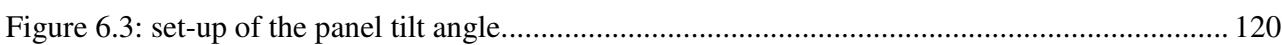

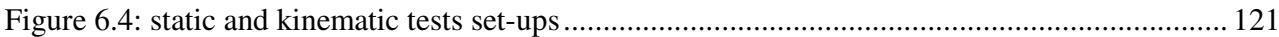

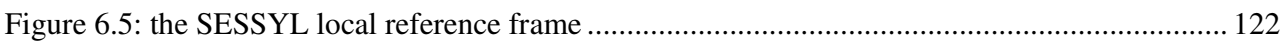

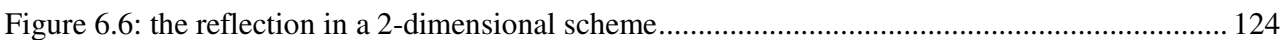

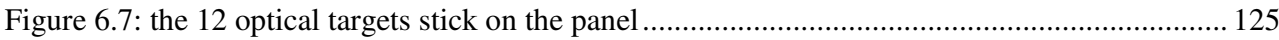

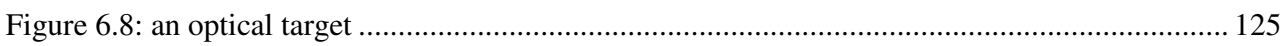

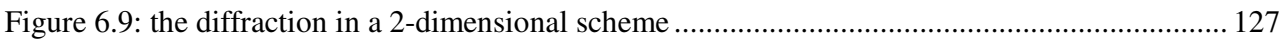

Figure 6.10: sky plot display of multipath zones, when the panel is placed 1, 4 and $7 \mathrm{~m}$ (the colour bar corresponds to the additional path length travelled by the reflected signal, in $\mathrm{m}$ ). 128

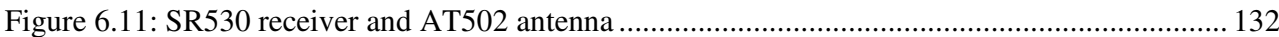

Figure 6.12a: day148 - standard deviation between the O-C DD and the model, versus $\alpha$ and $\Theta \ldots 134$ 
Figure 6.12b: standard deviation between the O-C DD and the model, versus $\alpha$, $\Theta$ fixed. 134

Figure 6.12c: modelled multipath phase error superimposed to the O-C DD. ................................. 134

Figure 6.13a: day210 - standard deviation between the O-C DD and the model, versus $\alpha$ and $\Theta \ldots 135$

Figure 6.13b: standard deviation between the O-C DD and the model, versus $\alpha, \Theta$ fixed. .............. 135

Figure 6.13c: modelled multipath phase error superimposed to the O-C DD. ................................ 135

Figure 6.14a: day211 - standard deviation between the O-C DD and the model, versus $\alpha$ and $\Theta \ldots 136$

Figure 6.14b: standard deviation between the O-C DD and the model, versus $\alpha, \Theta$ fixed. .............. 136

Figure 6.14c: modelled multipath phase error superimposed to the O-C DD. ................................. 136

Figure 6.15a: day212 - standard deviation between the O-C DD and the model, versus $\alpha$ and $\Theta \ldots 137$

Figure 6.15b: standard deviation between the O-C DD and the model, versus $\alpha, \Theta$ fixed. .............. 137

Figure 6.15c: modelled multipath phase error superimposed to the O-C DD. ................................. 137

Figure 6.16: AT502 gain patterns for L1 phase (AeroAntenna) .................................................... 139

Figure 6.17: reflection and diffraction geometrical computation ................................................... 141

Figure 6.18: static test with the panel at $4 \mathrm{~m}$ (8 SVs displayed only) - PMMW corrections disabled

Figure 6.19: static test with the reflector at $4 \mathrm{~m}$ (8 SVs displayed only) - PMMW corrections enabled

Figure 6.20a: effect of PMMW on phase DD in static mode (day210 - panel at $1 \mathrm{~m}$ ) ...................... 144

Figure 6.20b: effect of PMMW on phase DD in static mode (day211 - panel at $4 \mathrm{~m}$ ) ....................... 144

Figure 6.20c: effect of PMMW on phase DD in static mode (day212 - panel at $7 \mathrm{~m}$ ) ...................... 144

Figure 6.21: unability of PMMW to mitigate diffraction .............................................................. 149

Figure 6.22: the SESSYL platform, covered by the foam, and the panel at $8 \mathrm{~m} . . . \ldots \ldots \ldots \ldots \ldots \ldots \ldots \ldots . . . . . . . . . . . . . .150$

Figure 6.23: kinematic test with the panel at $1 \mathrm{~m}$ (8 SVs displayed only) - PMMW disabled ......... 152

Figure 6.24: kinematic test with the reflector at $1 \mathrm{~m}$ (8 SVs displayed only) - PMMW enabled ..... 153

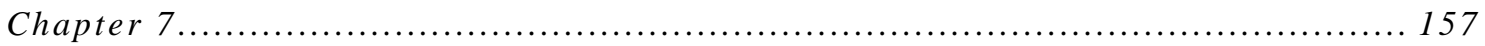

Figure 7.1: code MMW error envelopes for different ratio of amplitude.......................................... 158

Figure 7.2: the PRN code autocorrelation function in the interval $[ \pm 1$ chip $]$................................. 160

Figure 7.3: the discrimination function of the narrow correlator in the interval $[ \pm 1$ chip $]$.............. 161

Figure 7.4: multipath code error envelopes for different ratios of amplitude corresponding to the

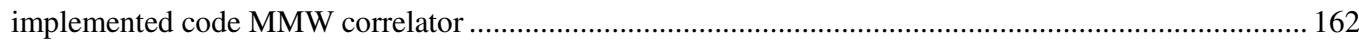

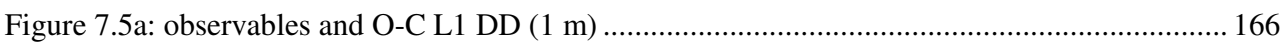

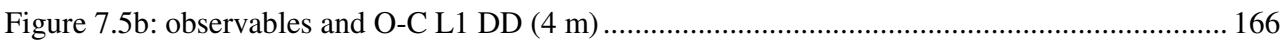

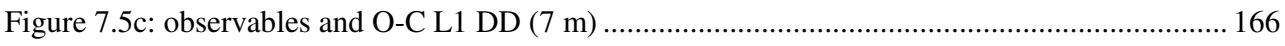

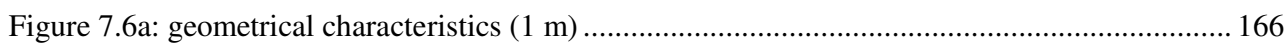

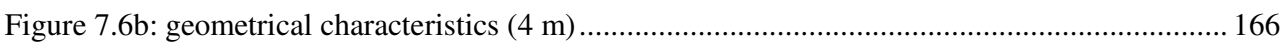

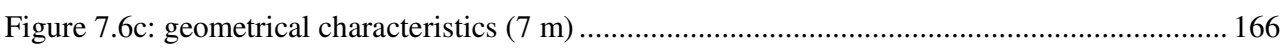

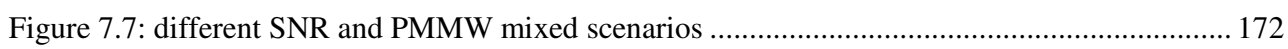

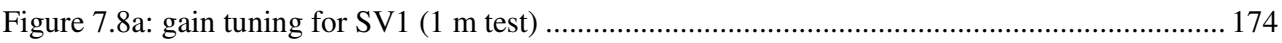


Figure 7.8b: gain tuning for SV1 (4 m test)

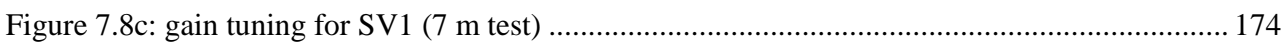

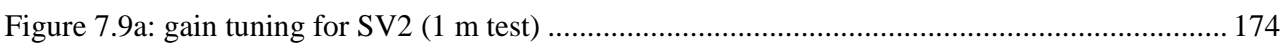

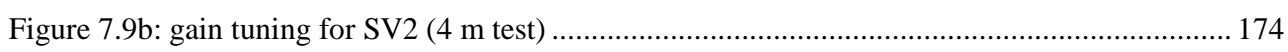

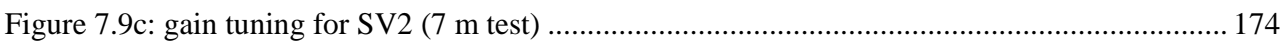

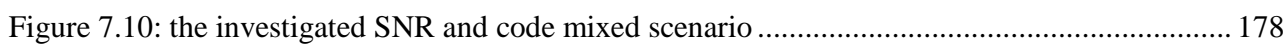

Figure 7.11a: SNR and code mixed scenario compared to SNR and PMMW/SNR mixed scenarios in the case of a very close reflector, i.e. $1 \mathrm{~m}$ distant (SV1 and SV2).

Figure 7.11b: SNR and code mixed scenario compared to SNR and PMMW/SNR mixed scenarios in the case of the $4 \mathrm{~m}$ distant reflector (SV1 and SV2)

Figure 7.11c: SNR and code mixed scenario compared to SNR and PMMW/SNR mixed scenarios in the case of the $7 \mathrm{~m}$ distant reflector (SV1 and SV2) .....

Figure 7.12a: SNR, code error and PMMW mixed scenario in the case of a very close reflector, i.e. 1 $\mathrm{m}$ distant (SV1 and SV2)

Figure 7.12b: SNR, code error and PMMW mixed scenario in the case of the $4 \mathrm{~m}$ distant reflector (SV1 and SV2)

Figure 7.12c: SNR, code error and PMMW mixed scenario in the case of the $7 \mathrm{~m}$ distant reflector (SV1 and SV2)

Figure 7.13a: reconstructed error (SV1 $1 \mathrm{~m}$ test) ….................................................................... 182

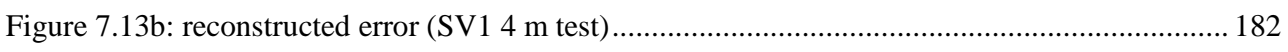

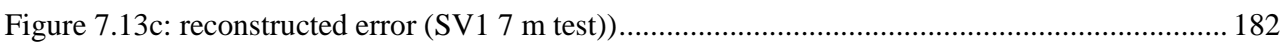

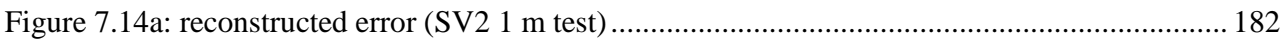

Figure 7.14b: reconstructed error (SV2 $4 \mathrm{~m}$ test) ........................................................................ 182

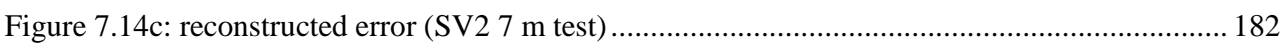

Figure 7.15a: reconstructed multipath phase errors for SV1 and differencing SV20 (left figure) and combination of both in a global correction of the O-C DD of phase (1 m test).....

Figure 7.15b: reconstructed multipath phase errors for SV1 and differencing SV20 (left figure) and combination of both in a global correction of the O-C DD of phase (4 m test).

Figure 7.15c: reconstructed multipath phase errors for SV1 and differencing SV20 (left figure) and combination of both in a global correction of the O-C DD of phase ( $7 \mathrm{~m}$ test).

Figure 7.16a: reconstructed multipath phase errors for SV2 and differencing SV3 (left figure)....... 188

and combination of both in a global correction of the O-C DD of phase $(1 \mathrm{~m}$ test $)$.......................... 188

Figure 7.16b: reconstructed multipath phase errors for SV2 and differencing SV3 (left figure) ...... 188

and combination of both in a global correction of the O-C DD of phase (4 m test)......................... 188

Figure 7.16c: reconstructed multipath phase errors for SV2 and differencing SV3 (left figure)....... 188

and combination of both in a global correction of the O-C DD of phase $(7 \mathrm{~m}$ test $)$.......................... 188

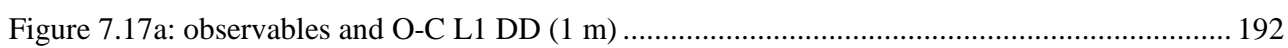

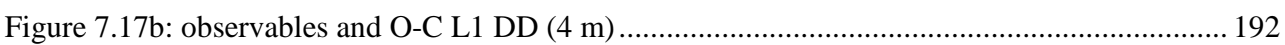

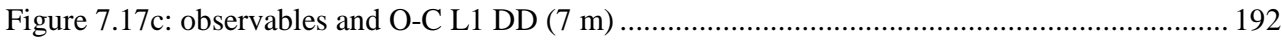


Figure 7.18a: reconstructed multipath error $(1 \mathrm{~m})$

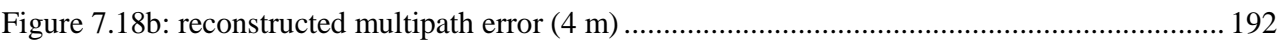

Figure 7.18c: reconstructed multipath error $(7 \mathrm{~m})$................................................................. 192

Figure 7.19: observables used in the reconstruction process for SV17 (4 m test) ............................ 197

Figure 7.20: reconstructed multipath phase error for SV17 (left figure) and combination with that for SV29 in a global correction of the O-C DD of phase $(4 \mathrm{~m}$ test) ............................................................ 197

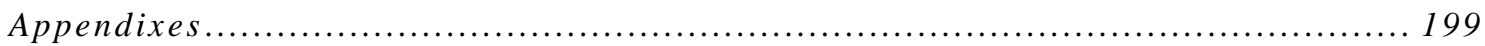

Figure A2.1: parallel and perpendicular reflection coefficients typical of water at hyperfrequencies.

Figure A2.2: parallel and perpendicular reflection coefficients typical of metal at hyperfrequencies.

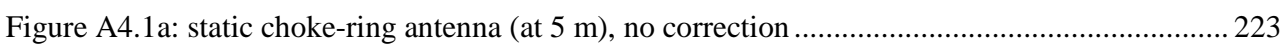

Figure A4.1b: static choke-ring antenna (at $5 \mathrm{~m}$ ), phase MMW correction applied .......................... 223

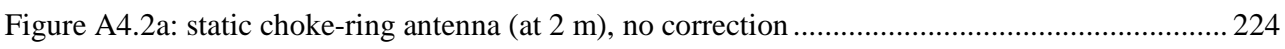

Figure A4.2b: static choke-ring antenna (at $2 \mathrm{~m}$ ), phase MMW correction applied .......................... 224

Figure A4.3a: static lightweight antenna (at $5 \mathrm{~m}$ ), no correction ................................................... 225

Figure A4.3b: static lightweight antenna (at $5 \mathrm{~m}$ ), phase MMW correction applied......................... 225

Figure A5.1: the lightweight antenna on the SESSYL platform ...................................................... 226

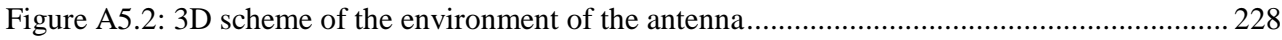

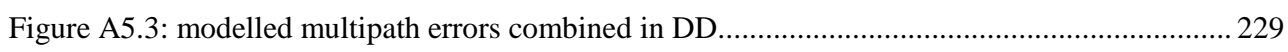

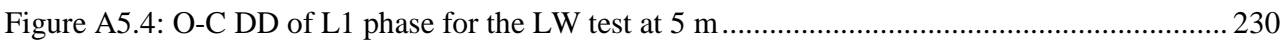

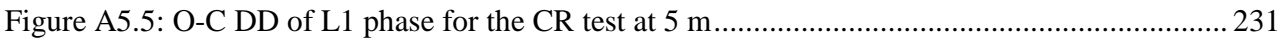

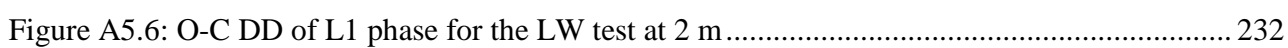




\section{TABLE}

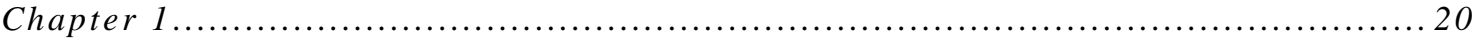

Table 1.1: attenuation and phase shift for reflection from a metallic material .................................... 25

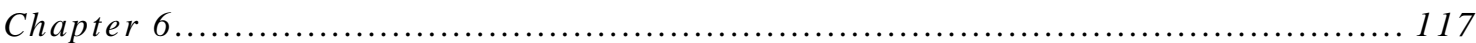

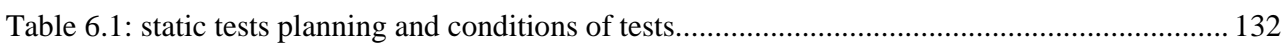

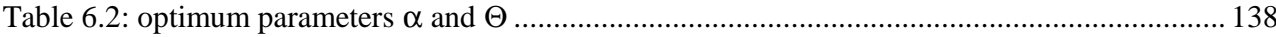

Table 6.3a: statistical results for day2 10 (panel at $1 \mathrm{~m}$ ) ............................................................ 146

Table 6.3b: statistical results for day211 (panel at $4 \mathrm{~m}$ )................................................................ 147

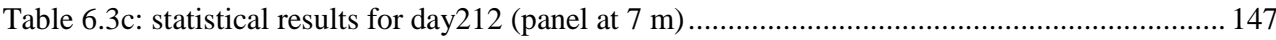

Table 6.4: L1 phase DD statistics in static mode .......................................................................... 148

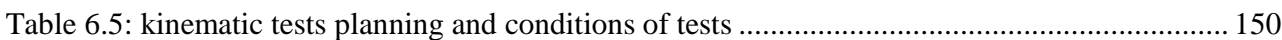

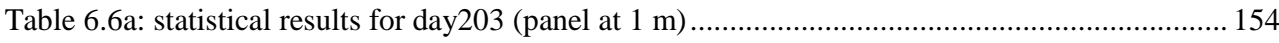

Table 6.6b: statistical results for day204 (panel at $4 \mathrm{~m}$ ).................................................................. 154

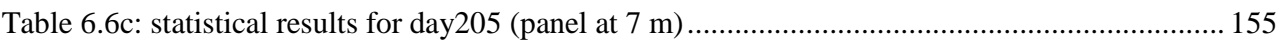

Table 6.7: L1 phase DD statistics in kinematic mode ............................................................... 155

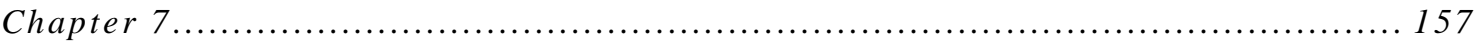

Table 7.1: optimal standard deviation and corresponding gain for different reconstruction scenarios

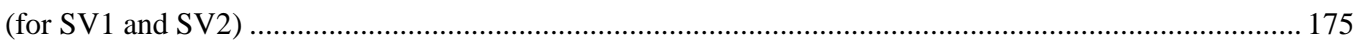

Table 7.2a: statistical results of the reconstruction for day210 (panel at $1 \mathrm{~m}$ ) ................................. 185

Table 7.2a*: same as table 7.2a, but SVS with elevation $>15^{\circ}$ only are included........................... 185

Table 7.2b: statistical results of the reconstruction for day211 (panel at $4 \mathrm{~m}$ ) ..................................... 186

Table 7.2c: statistical results of the reconstruction for day212 (panel at $7 \mathrm{~m}$ ) ................................. 186

Table 7.3: L1 phase DD statistics in static mode (all data in multipath zones) (for "standard", "PWWM corrected" and "reconstructed" phase data)

Table 7.4: L1 phase DD statistics in static mode (all data, all epochs) (for "standard", "PWWM corrected" and "reconstructed" phase data)

Table 7.5a: statistical results of the reconstruction for day203 (panel at $1 \mathrm{~m}$ ) 193

Table 7.5a*: same as table 7.5a, but SVS with elevation $>15^{\circ}$ only are included............................ 193

Table 7.5b: statistical results of the reconstruction for day204 (panel at $4 \mathrm{~m}$ ) ................................... 194

Table 7.5b*: same as table 7.5b, but svs with elevation $>15^{\circ}$ only are included............................... 194

Table 7.5c: statistical results of the reconstruction for day205 (panel at $7 \mathrm{~m}$ ) ................................. 194 
Table 7.6: L1 phase DD statistics in kinematic mode (for "standard", "PWWM corrected" and "reconstructed" phase data).

Table 7.7: L1 phase DD statistics in kinematic mode (all data, all epochs) (for "standard", "PWWM corrected" and "reconstructed" phase data) 


\section{NOTATION}

\begin{tabular}{|c|c|}
\hline $\mathrm{C} 1$ & $\mathrm{C} / \mathrm{A}$-code measurement on $\mathrm{L} 1$ \\
\hline $\mathrm{P}$ & P-code measurement \\
\hline$C(t)$ & received PRN code (either $\mathrm{C} / \mathrm{A}$ or $\mathrm{P}$ codes) time series \\
\hline $\mathrm{D}(\mathrm{t})$ & received GPS message data bit time series \\
\hline $\mathrm{R}$ & code autocorrelation function \\
\hline$\Phi$ & phase measurement \\
\hline$\lambda$ & carrier wavelength \\
\hline $\mathrm{dT}$ & clock offset of the receiver \\
\hline $\mathrm{dt}$ & clock offset of the satellite \\
\hline $\mathrm{Z}$ & tropospheric range delay \\
\hline $\mathrm{I}$ & ionospheric range delay \\
\hline M & multipath range error \\
\hline$\varepsilon$ & receiver noise \\
\hline c & speed of light \\
\hline $\mathrm{fn}$ & nominal frequency (L1 or L2) \\
\hline
\end{tabular}


fi intermediate frequency (i.e. nominal down-converted frequency)

f Doppler affected intermediate frequency

$\rho \quad$ geometric range between the satellite and receiver antennas

$\dot{\rho} \quad$ satellite-receiver antennas range rate

ASIC application specific integrated circuit

RF radio frequency

$\mathrm{N} \quad$ integer ambiguity

IF intermediate frequency (after down-conversion)

BW bandwidth

$\mathrm{T}$ duration of the C/A-code chip

L additional path length of the reflected signal

d code delay of the reflected signal (i.e. L/c)

$\alpha \quad$ ratio of amplitude of the direct and reflected signals

$\Theta \quad$ phase shift of the reflected signal

$\varphi_{\mathrm{m}} \quad$ multipath phase error

$\tau_{\mathrm{m}} \quad$ multipath code error 
Chapter 1

\section{INTRODUCTION}

This chapter introduces the background of the research carried out in the frame of this thesis. It also gives general statements about the physical description of the phenomenon of multipath.

\section{1- Background of the work}

Despite enormous developments in Real Time Kinematic GPS over the past decade its practical application is still often limited by the presence of errors due to multipath. These errors not only limit the precision that can be obtained but also put restrictions on ambiguity resolution, which can, amongst other things, have the effect of limiting operational range. Whilst much progress has been made in limiting the impact of multipath on pseudo-range measurements (both within the receiver and in data processing), there has been less success in limiting multipath effects on carrier phase measurements. These are the basis of RTK GPS, and many applications in civil engineering (like road pavement laying) still require a level of precision that is not attainable without further improvement in phase multipath mitigation.

\section{2 - Aspects of the theory of multipath}

Multipath encompasses different phenomena related to the propagation of the electromagnetic waves [BRAASCH, 1996]. These can be divided into two categories: reflection and diffraction. This chapter enlightens the physical characteristics of both. 
Contrary to atmospheric effects on GPS signal propagation (i.e. tropospheric and frequency dependent ionospheric refractions of L1 $1575.42 \mathrm{MHz}$ and L2 $1227.60 \mathrm{MHz}$ carrier waves) or orbit uncertainties, multipath is essentially station dependent and remains sensitive for short baselines. It is generally admitted that, because of their spatial correlation, errors caused by the atmosphere (except very localised ionospheric effects such as scintillations) and orbits cancel over the very short baselines that are common in engineering applications of GPS. This is not true for multipath errors, which are generally completely different at the base and at the rover station.

\subsection{1 - Origin of multipath and local model of propagation}

Reflections take place when the waves hit obstacles, like the surface of water or building structures, and, as a consequence, propagate indirectly from the emission point to the reception point. The reflection of electromagnetic waves is a phenomenon comparable to the echo of acoustic waves.

GPS signals are prone to multipath from the local environment of the antennas. As represented in Fig. 1.1, multipath can be due to reflecting surfaces in the environment of both the reception and the emission of the signals, since the satellite itself can create multipath.

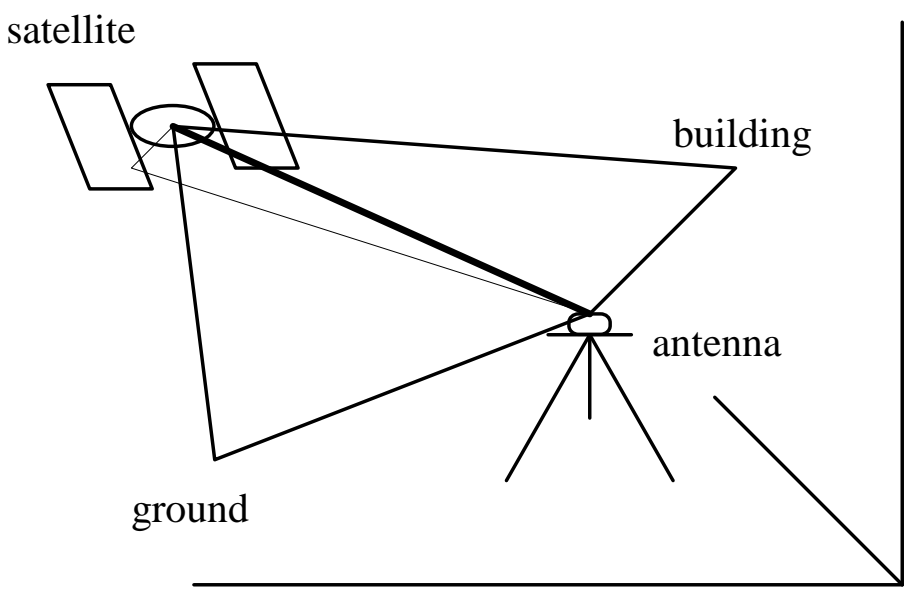

Figure 1.1: direct path and reflected paths 
Note that any reflection due to a satellite will have the same effect on a pair of receivers in a local network [YOUNG et al., 1985], and it has no consequence in differential positioning (it cancels between the receivers in the network). Thus multipath that is satellite originating will not be considered in this thesis.

Certain surfaces, plane and conductive, are particularly likely to create multipath in the environment of a GPS antenna. These surfaces, contrary to those without such geometrical and electrical characteristics, reflect the signal by preserving its waveform. In this case, the signal reflection is denoted "specular", as opposed to "diffuse".

The roughness and the size of the surface where a reflection may take place will determine whether this is specular or diffuse. A criterion, known as the Rayleigh criterion, describes how rough a surface is, with respect to the wavelength:

$\lambda / 4>\sigma h \sin \theta$

where

$\sigma \mathrm{h}$ is the mean height of the irregularities located on the surface of the reflector $\lambda$ is the wavelength (L1 or L2 for GPS), and

$\theta$ is the angle of elevation with respect to the surface, also called the grazing angle (equal to $90^{\circ}$ - the angle of incidence).

In the frame of this research and particularly as concerns the experiments carried out, it is always a priori supposed that the Rayleigh criterion is satisfied. Most surfaces in a typical environment in civil engineering (buildings, ground, engineering sites, metallic surfaces of machines...) are indeed smooth enough to be considered as specular reflectors.

If it is required to model the phenomenon of multipath by geometrical methods then another criterion, additional to that of Rayleigh, must also be considered. This concerns the size of the reflectors with respect to the wavelength of the carrier. In the case of GPS signal propagation, it is generally considered that the wavelengths (L1: $19 \mathrm{~cm}$ and L2: $24 \mathrm{~cm}$ ) are small in comparison with the dimensions of the obstacles in the environment. 
Note that ray-tracing techniques are based on the same two criteria, and these techniques provide models and tools classically accepted as valid for GPS multipath.

Assuming the two criteria to be satisfied, the reflection is deterministic, and the SnellDescartes law of propagation used in geometric optics can also be used to describe reflection of the GPS signal.

Hence, an incident wave (represented by vector I in Fig. 1.2) on the surface of a material with a complex dielectric constant $\varepsilon$, is reflected in the direction $\mathrm{R}$ such that vector $\mathrm{R}$ is contained in the plane formed by vector I and vector $\mathrm{N}$ normal to the surface:

$$
\text { angle }(-\mathrm{I}, \mathrm{N})=\text { angle }(\mathrm{N}, \mathrm{R})
$$

i.e. the angle of reflection is the same as the angle of incidence.

Note that in general with dielectric materials the incident wave is also transmitted in the direction $\mathrm{T}$ such that vector $\mathrm{T}$ is again contained in the same plane, with a certain refraction angle with respect to vector $\mathrm{N}$. The transmitted energy is much lower than that reflected with most current materials (water, ground, concrete, metal...). Note that refraction is the phenomenon that occurs when the GPS waves propagate through the different layers of the atmosphere. This phenomenon will not be addressed further in this thesis.

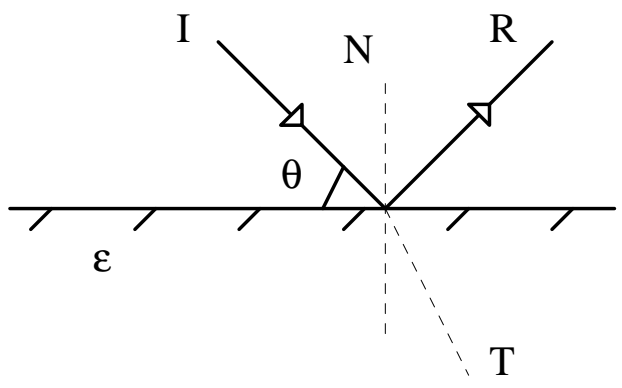

Figure 1.2: reflection and transmission in geometric optics

In a short baseline network, signal reflection is not the only cause of station dependent errors in measurements. Signal diffraction is another phenomenon that typically occurs 
when satellites are rising or setting, or when the line of sight hits obstacles surrounding the antenna (the horizon, the relief, a tree, the corner of a building, the edge of a structure...). Diffraction corresponds to a diffusion of the direction of propagation of the electromagnetic waves around the obstacles. This phenomenon causes measurements to be possibly made on satellites that are not in direct line of sight, but whose signals have diffracted or in other words apparently bent around the obstacles. Note that the "elevation mask" set-up in a receiver permits satellites that are below a certain elevation angle to be disabled. However, when the horizon is above this angle set-up, measurements from signals that may be diffracted are used in the positioning process.

\subsection{2 - Multipath effects on the signal}

Reflection modifies the spectral parameters of the signal, mainly in amplitude and phase, and also frequency if the antenna moves in the environment. Electromagnetic theory provides a comprehensive description of the phenomenon. The general concept to bear in mind is the fact that the coefficient of reflection (that links both amplitude and phase of the incident and reflected waves) depends on the material of which the reflector is made. Moreover, this coefficient is a complex quantity that depends on the grazing angle and the complex dielectric constant $\varepsilon$ of the material. Its magnitude and its argument respectively affect the amplitude and the phase of the reflected signal.

The main point is that reflection causes a "primary" attenuation of the amplitude of the signal, because the magnitude of the coefficient of reflection is always less than 1.

The coefficient of reflection has noticeable properties with respect to an incident linearly polarised wave tests (see Appendix 2). This is the case whether it is horizontal (i.e. if the electric field vector $\mathrm{E}$ is in the plane of the surface) or vertical (i.e. if vector $\mathrm{E}$ is in the plane formed by vector I and vector N). A transverse electromagnetic (TEM) wave (i.e. a wave whose electric field is perpendicular to the direction of propagation - a GPS wave is TEM) can always be split up into two components, vertically and horizontally polarised. The attenuation is generally not the same for both, neither the phase shift, which explains the change of polarity. The phase shift is approximately $180^{\circ}$ for the horizontal 
component, and 0 or $180^{\circ}$ for the vertical component, depending on whether the grazing angle $\theta$ is above or below the Brewster angle (an angle that also depends on the material).

In general, for GPS signals, the circular polarity $(\mathrm{CP})$ of the incident wave is lost after a reflection: it becomes elliptical (EP). This sign of the polarity (RHCP for Right Hand CP) may be inverted (LHEP for Left Hand EP) if the grazing angle is above the Brewster angle.

The next table summarises the main properties that relate to the attenuation and phase shift for reflection from a metallic material (the reflectors used for the experiments carried out in the frame of this research were made of metal). Other materials have different properties, and theses like [MALICORNE, 2001] or [HANNAH, 2001] provide quite comprehensive descriptions of these, for civil engineering environment.

\begin{tabular}{lll}
\hline & horizontal & vertical \\
\hline attenuation & $\sim 1$ & $\sim 1$ \\
phase shift & $180^{\circ}$ shifted & not shifted, $\theta>\theta b$ \\
& & $180^{\circ}$ shifted, $\theta<\theta b$
\end{tabular}

Table 1.1: attenuation and phase shift for reflection from a metallic material

$\theta \mathrm{b}$ denotes the Brewster angle. The value of this angle, in the case of a metallic reflector, is around 1 hundredth of a degree (see Appendix 2).

Furthermore, the sign of the polarity (i.e. the rotation of the electric field) is inverted if the grazing angle is above the so-called Brewster angle. As a consequence, the phase of the reflected signal shifts by $180^{\circ}$. In the experiments carried out and reported in this thesis, the grazing angle was always above the Brewster angle, which can be considered to be zero. So, one can consider that there is always a "reflection phase shift" of $180^{\circ}$.

Note that below the Brewster angle, both horizontal and vertical components are $180^{\circ}$ shifted in phase, which results in no phase shift for the reflected signal. 
Concerning the frequency, if the relative geometry of the receiver antenna and a reflector is changing, for instance because of a movement of the receiver antenna in kinematic applications, then the frequency of the reflected signal is affected by a Doppler shift, due to the relative movement. In the frame of most high-precision applications of RTK GPS in machines guidance and site robotics, the change of the frequency of the reflected wave can be neglected since the relative movement is generally at low speed (a few $\mathrm{km} / \mathrm{h}$ maximum).

\subsection{3 - Gain and phase patterns of the antenna}

In addition to the primary attenuation and the reflection phase shift, the gain and phase patterns of the antenna need to be taken into account (i.e. the amplitude and phase variations applied to the signal by the antenna itself). These are different for right and left polarisations, and also dependent on the elevation and azimuth of the signal on the plane of the antenna. Note that a well-known consequence in geodesy of the phase pattern is the so-called "phase centre variation".

This gain pattern causes a "secondary" attenuation of the amplitude of the signal because most GPS antennas are Right Hand Circularly Polarised by construction and if they do not reject, they at least attenuate Left Hand Elliptical Polarised waves.

Lastly, the phase pattern creates an "antenna phase shift" that further modifies again the phase of the signal, particularly if it is left-hand polarised.

In conclusion, one can assume that in the case of reflectors made of metal (such as might be found on an engineering site), the reflection is specular, and the grazing angles are always above the Brewster angle. Hence, the relative phase between the reflected signal and the direct signal is only dependent on the additional path length, plus the $180^{\circ}$ reflection phase shift, plus the antenna phase shift. The attenuation corresponds to that of a LHCP received signal. The ray-tracing modelling used further in this thesis is based on this assumption. 
1.2.4 - Representation of the GPS received signal in the receiver

The following representation of the received direct line of sight signal in the receiver is widely used in this dissertation and generally in the literature:

$\mathrm{S}=\mathrm{AD}(\mathrm{t}) * \mathrm{C}(\mathrm{t}) * \sin (\Phi(\mathrm{t}))$

where

A is the amplitude of the received signal

$\mathrm{D}$ is the received GPS message data bit time series

$C$ is the received PRN code time series, and

$\Phi$ is the phase of the received signal $\left(\Phi(t)=2 \pi^{*} f^{*} t\right.$ where $f$ is the carrier frequency).

A few comments are worth making with regard to this representation.

First, it is simplified (it does not show the two carrier frequencies neither the different codes C/A and P, see Chapter 3, §3.1). But it is generally adequate to demonstrate the principles of multipath mitigation techniques. Furthermore, it specifically addresses the component of the received signal that corresponds to a single satellite. Several components like this are actually mixed together. They are identified in the signal processing carried out by the receiver.

$D, C$ and $\Phi$ are the time series of the message, the pseudo-random code and the carrier phase, each of them affected by Doppler and propagation effects. These effects are not detailed in this representation. One can assert that, when received, the original synchronisation of these time series, i.e. when emitted, has changed somehow while the signal was travelling. This is detailed in Chapter 3 where the functioning of a standard receiver is introduced.

Lastly, the notion of polarity has disappeared in this representation of the received signal. Actually, this representation addresses the received signal in the receiver, i.e. after the antenna, and not before. So it corresponds to the measurement of the electric field by the antenna. And in fact the polarity of the signal appears indirectly in this representation: 
- in the amplitude A through the antenna gain pattern (see Chapter 2, § 2.1.1), and

- in the phase $\Phi$ since the measurement of the electric field by the antenna would instantaneously shift in phase by $180^{\circ}$ if the polarity of the electric field was inverted at this instant.

\subsection{5 - Overall consequences of multipath in the range measurements}

When a GPS receiver is tracking a signal emitted by a GPS satellite, it cannot discriminate whether this signal is coming directly from the satellite (direct path), or whether it is also composed of one (or several) reflection(s) of that signal (reflected path(s), also called indirect). The direct path and the reflected path(s) interfere together because their code modulation is the same (contrary to different satellites that cannot interfere, see Chapter 3, §3.1). This multipath resulting interference causes code and carrier phase tracking errors, which have a direct influence on the GPS raw measurements (i.e. range measurements), and consequently on the positioning results.

The reflected signal will definitely contaminate the direct signal in a specific way, which results in errors in measurements that are obviously different from an additive white noise (such as receiver noise). Multipath errors in measurements are coloured (they are correlated with themselves). Their periods are rather long: 1 minute to 1 hour, either in code or phase data, depending on how the distances to the surrounding reflectors vary. Their amplitudes are unknown a priori. As concerns the range errors that correspond to code measurements, a bound of a certain fraction of the chip length exists, and it depends on the correlator used to process the code measurements. With a wide correlator, these errors are within some ten metres (for $\mathrm{C} / \mathrm{A}$-code) and within some metres with a narrow correlator (see Chapter 4, Fig. 4.5). The range errors that correspond to phase measurements are limited to one quarter of the wavelength, i.e. a maximum of a few centimetres for GPS L1 and L2 wavelengths. These bounds and periods are explained in detail in Chapter 4.

From the point of view of the GPS user, multipath on code measurements may increase the positioning error by some metres. This is an order of magnitude, for receivers 
using a narrow correlator. Of course, it is highly dependent on the number and the geometry of the satellites combined in the least squares process of positioning.

Positioning based on phase data may show errors of a few centimetres with modern receivers. The number and the geometry of the satellites is also highly determinant in the level of the final positioning errors.

Note: for multipath, one generally assume that direct and reflected signals are mixed and fed together to the signal tracking process. This hypothesis may be wrong, when the direct signal is masked. Then, code and phase errors are theoretically unbounded (they may increase by the additional path length of the affected signal while the receiver tracks the signal).

In case of diffraction of the signal for a given satellite, the diffracted signal is generally the only one fed to the corresponding signal tracking process. This means that, in the case of diffraction, there is no interference as described in the case of reflection. In other words, the direct signal is a diffracted signal. This signal is delayed and phase shifted. Diffraction can cause much larger errors than reflection in terms of amplitude (the bias in range measurements is only bounded by the additional path length of the affected signal), with duration generally shorter. Actually, satellites causing diffracting waves are very specifically located with respect to the obstacles at the horizon, whereas there is never a totally multipath free environment and waves are reflecting more or less permanently.

The time series in Fig. 1.3 come from [WIESER and BRUNNER, 2000], whose mitigation theory is presented in Chapter 2. This figure displays the vertical component of a static survey during several hours. 
The top graph superimposes two time series, the original one ("dark") has no mitigation of the diffraction. The second and third time series are zooms on a severe diffraction error. Once mitigated, there only remain errors due to reflections.
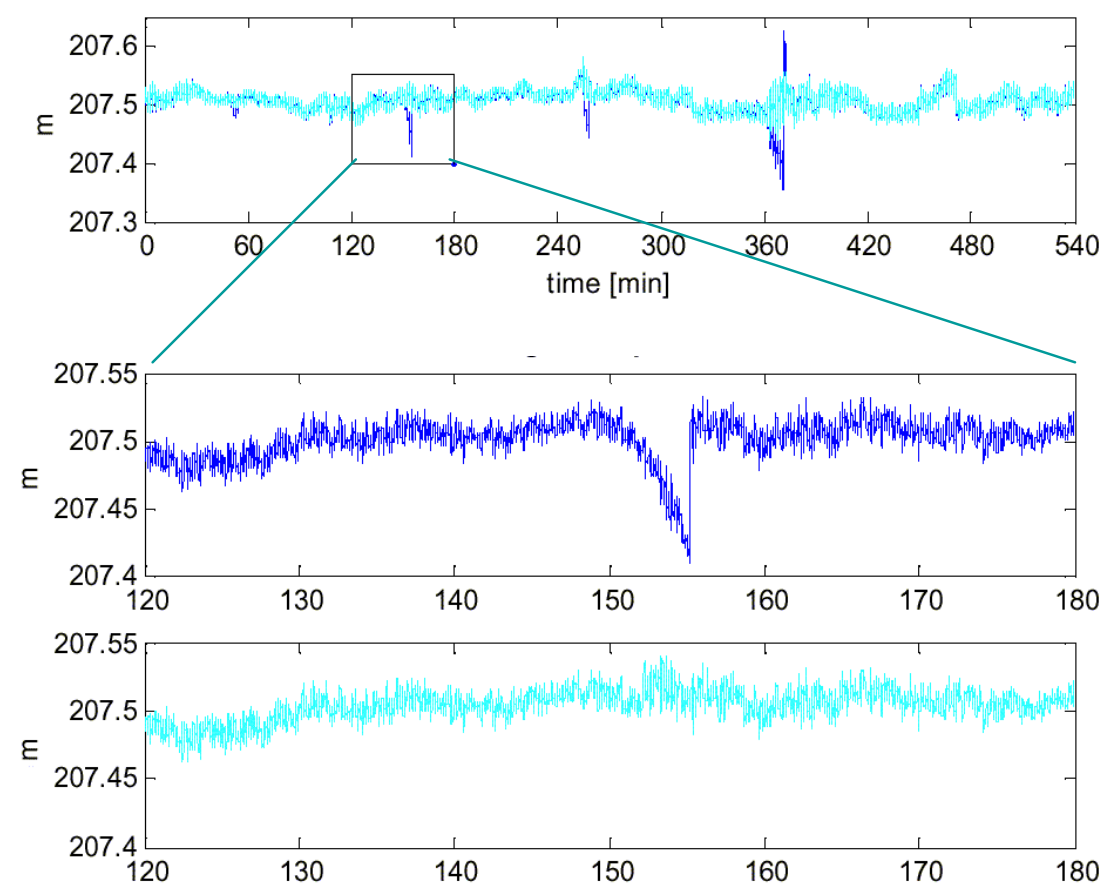

Figure 1.3: static survey showing both diffraction and reflection

Roughly, in short baseline static survey (when local ionospheric effects are neglected) one can say that long term deviations (bounded in amplitude) are symptomatic of reflection. On the contrary, short-term deviations with much larger amplitude may be due to diffraction from a satellite with low elevation generally.

In kinematic applications, the obstacles that are moving with the receiver antenna cause multipath very similar as that observed in static applications. The reflectors that are not static in the body-fixed frame of the antenna may cause multipath with shorter duration, this depending on the changing of the antenna-environment relative geometry. Maximum amplitudes of multipath errors are the same in static and kinematic modes. 


\section{3 - Conclusions}

The phenomenon of multipath is related to local obstacles from which the electromagnetic waves rebound. Typical reflectors are buildings, water planes, metallic panels on machineries... all showing basically various physical properties in relation with this phenomenon. Diffraction, like reflection, is another local electromagnetic phenomenon, with other characteristics.

The interference that results from multipath causes code and carrier phase tracking errors that depend on the design of both the antennas and the correlators implemented in the ASIC (application specific integrated circuit). This point is detailed further in this thesis.

Multipath results in errors in the measurement of ranges. In differential GPS, it is the main error source in the error budget for short baselines, i.e. baselines of a few kilometres, because it has totally specific and different effects at the base and at the rover (contrary to tropospheric and ionospheric delays, as well as orbit uncertainties, that are highly spatially correlated in this case). Note that local ionospheric effects are possible, particularly in case of ionospheric storms.

Positioning by satellites results from least squares processing of a number of individual distances along lines of sight between receivers and satellites antennas, generally combined as double differences (DD). Hence, it may not be trivial to identify clearly individual multipath ranging errors in positioning solutions (neither due to reflection nor diffraction), particularly when there are a large number of satellites. In this case, multipath is visible in the residuals of the least squares process of positioning and impact only moderately the positioning solution. Two situations are rather critical with respect to the positioning solutions: first, if multipath concerns satellites at high elevation, and/or second, in case of a poor constellation. Actually, if the highest elevated satellite, that is generally chosen for differencing, is affected by multipath, then every DD is contaminated. And lastly, multipath errors are more visible in positioning solutions if only a few satellites are combined in the least squares processing. 
Chapter 2

\section{MAIN RESULTS IN MULTIPATH MITIGATION}

This chapter gives a brief review of the literature that deals with GPS multipath mitigation. The antenna based mitigation is examined first, then the receiver based mitigation, which basically consists in improving the measurements by original correlation techniques, and lastly the multipath mitigation by data processing.

\section{1- Antenna based mitigation}

\subsection{1 - Specific features of standard GPS antennas related to multipath}

Modern GPS units first carry out signal amplifying/attenuating in the antenna, before any other filtering and digital signal processing in the receiver.

The characteristics of standard GPS antennas that specifically relate to multipath are reported here. These are designed to be selective with the polarisation of the received wave.

Multipath affects the polarisation of GPS waves. GPS electromagnetic signal is righthand circularly polarised (RHCP), and this property is modified by reflections. A reflected signal will be roughly elliptically polarised, and, depending on the incidence and the properties of the material, the right polarity can be inversed (LHEP). The sensitivity of GPS antennas to left-hand polarised waves has been of much interest in the past when much of the original design work was carried out.

In a GPS antenna, the signal is either amplified or attenuated as a function of the elevation of the satellite relative to the plane of the antenna, and also as a function of the 
azimuth for a non micro-centred antenna. The spatial variation of the sensitivity of an antenna is called the antenna gain pattern. There is a different gain pattern for each polarisation of the wave. A description of the antenna that includes not only the diagram for right-hand polarity but also for left-hand is necessary to model completely the direct signal amplification and the reflected signal attenuation in case of multipath.

Gain patterns are generally available from GPS manufacturers or laboratories equipped with an anechoic chamber [SCHUPLER, 2001]. Note that the diagrams change if a ground plane is added to the original antenna.

If the antenna gain pattern is not available, one can estimate it by fitting a polynomial to signal-to-noise ratio data as a function of the elevation of the satellite. To do so, it is recommended to set up the equipment in an area free of multipath. Such a calibration should be done over a complete 24 hours session of observation. Note that by doing so, only a right-hand diagram is obtained.

\subsection{2 - Choke-ring antennas}

Let us recall the principle of a choke-ring antenna (see Fig. 2.1). It is a ground-plane (metallic plate under the antenna itself), which quite effectively prevents the antenna from multipath due to reflections below the horizon of the antenna, such as reflections on the ground or on water. Despite its particular gain pattern, the ground-plane antenna will still be sensitive to multipath. The main reason for that is the propagation of the reflections along the top surface of the plate onto the centre of phase. To prevent this phenomenon, choke-ring antennas are also composed of metallic rings on top of the plate that are designed to attenuate that propagation. Rings are separated by one quarter of the wavelength (L1 or L2) [WEILL, 1997]. More recent development brought about with choke-ring antenna concept available for both L1 and L2 signals [FILIPPOV et al., 1999]. 


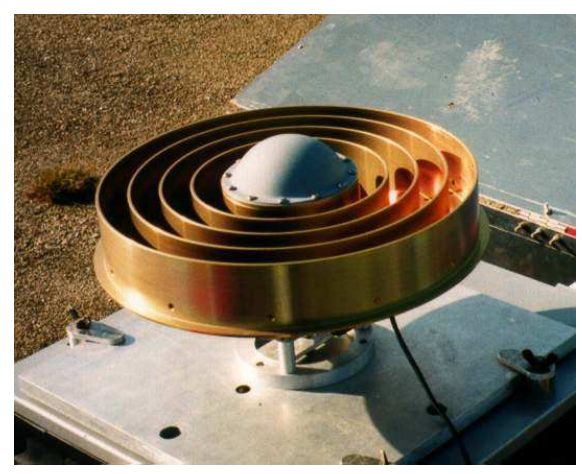

Figure 2.1: a choke-ring antenna

To conclude, a total efficiency in multipath mitigation by antennas with ground-planes and choke-ring antennas is not guaranteed. Furthermore, by their principle, they do not reduce the multipath due to reflections above the horizon of the antenna. Tests that were done in the frame of this thesis with Leica AT540 choke-ring antennas (see Appendix 4) showed multipath phase errors (on ranges) twice less than those with AT502 lightweight antennas. But their amplitude is still near $1 \mathrm{~cm}$.

\subsection{3 - Other antennas}

It is beyond the scope of this thesis to undertake a comprehensive investigation into the field of antenna technology. Further reading is referenced in proceedings or literature collections [BRODIN, 2001]. This section only mentions the innovations in antenna technology, particularly for high precision applications, that have taken place in several projects such as:

- Shorted Annular Patch (ESA project): this small antenna $(10 \mathrm{~cm})$ includes a cylindrical conducting wall the dimensions of which enable the gain pattern to be tuned. This pattern has a RHCP gain ranging from -10 to $-33 \mathrm{~dB}$ for elevations less than $20^{\circ}$ and a $\mathrm{LHCP}$ gain of -17 to $-25 \mathrm{~dB}$ in this region. The ratio of only $7 \mathrm{~dB}$ at $20^{\circ}$ between the two polarities is not better than that of lightweight antennas commonly used on civil engineering GPS applications (see the AT502 gain pattern in Chapter 6, Fig. 6.16, where this ratio is at least of $10 \mathrm{~dB})$. 
- Microstrip Array on a Resistivity Tapered Ground Plane (MTRE Corporation project): a microstrip patch, mounted on a ground plane, enables LHCP attenuation to be significantly improved. The ratio of RHCP gain to LHCP gain is of 12 to $20 \mathrm{~dB}$ for elevation angles less than $15^{\circ}$. But the design of this antenna only optimises a pre-selected elevation and azimuth, which is not suitable if there is no dominant reflector known a priori. The $50 \mathrm{~cm}$ size of the prototype was reduced to $10 \mathrm{~cm}$ in a new version.

- Phased Arrays and Beam Forming (NAVSYS Corporation project): this technique provides an adaptive tuning of the gain pattern of a $4 \times 4$ array of patches. This pattern can be dynamically attenuated by $40 \mathrm{~dB}$ in the elevation and azimuth of a multipath source. Only limited results have been reported so far, and the problem of the determination of the actual centre of phase of the array is not addressed. The size of this array is half a metre.

Most of these techniques based on antenna design are still under development. Moreover they do not solve completely the problem of multipath mitigation. What they all suggest is an optimisation of the gain selectivity. So, measurements that enter the receiver are less, but still, corrupted.

Moreover, for practical reasons, one of the constraints of the research carried out in the frame of this thesis is to use a single existing antenna, lightweight if possible, in order to be easily mounted over vehicles and machineries.

\section{2 - Multipath mitigation by correlation techniques}

\subsection{1 - Code correlation}

In standard GPS receivers, the code tracking is generally based on a technique that combines a "wide" correlator for initial tracking and an advanced multipath mitigation correlator to maintain lock on the signal. Historically, the first multipath mitigation correlator was the "narrow" correlator. Both wide and narrow correlators are presented in detail in Chapters 3 and 4. 
Wide correlators use Early-Late code replicas separated by 1 chip. Narrow correlators use the same replicas separated generally by 0.1 chip. The multipath code error is divided by 10 in amplitude by narrowing $(7.5 \mathrm{~m}$ instead of $75 \mathrm{~m}$ for a half voltage amplitude multipath signal) and the maximum multipath delay that can cause a code tracking error is reduced from 1.5 to 1.05 chip (see Chapter $5, \S 5.1 .1$ ).

Another family of code correlators, described in Chapter 5, § 5.1, has been developed based on of the narrow correlator. These correlators are known by their commercial trademarks: Leica A and B "MM" correlators [HATCH et al., 1997] or "ClearTrak" [STANSELL and MAENPA, 1999], Ashtech "Strobe" correlator [GARIN and ROUSSEAU, 1996], Trimble "Everest", 1999]. They are also named "reference waveform" or "gated" correlators [McGRAW and BRAASCH, 1999]. They enable both multipath code error and maximum multipath delay to be reduced, for instance by a factor of 10 (compared to the wide correlator), and ever much more: e.g. a factor of 40 with Leica MM correlator (see Chapter 5, § 5.1.3 and § 5.1.4). With this last correlator, the amplitude of the multipath C/A-code error is $2 \mathrm{~m}$, and for multipath delay of $10 \mathrm{~m}$ maximum.

Another extension of the narrow correlator was presented by [TOWNSEND and FENTON, 1994]. The Multipath Elimination Technique (MET) improves the narrow correlator by providing an estimation of the slope of the sides of the autocorrelation function. MET, and also its more recent version: PAC (Pulse Aperture Correlator) produces similar results to reference waveform correlators.

The main limitation of the performance of reference waveform correlators depends on the sampling rate used in the receiver, that itself is limited by the pre-correlation bandwidth of the signal. This is explained in Chapter 5, §5.1.3. Note lastly that the code correlation techniques have almost no effect of the phase tracking error.

To conclude the topic of code multipath mitigation by correlation techniques, one can say that close reflectors (causing additional path length of a few metres) still create multipath in code measurements. Hence, the actual error carried out by multipath on code measurements is of the order of a few metres with C/A-code (typically $2 \mathrm{~m}$ with the Leica 
MM correlator). The maximum multipath delay is around $10 \mathrm{~m}$. These values correspond to Leica MM correlator with a $40 \mathrm{MHz}$ clock rate.

The same technique applies to $\mathrm{P}(\mathrm{Y})$-code, but the level of improvement of reference waveform correlators (compared to the wide correlator) is not as important as with C/Acode, because of the noise caused by the decryption process.

\subsection{2 - Phase correlation}

A technique applicable to phase measurement was introduced by [VAN NEE, 1992]: Multipath Estimating Delay Lock Loop. MEDLL uses multiple correlators designed to track not only the direct signal but also the reflected signals. It was developed by Delft University and then implemented in Novatel receivers. A de-convolution is processed for each line of sight, which enables separation of the multiple signals interfering with the nominal one. MEDLL has been compared to standard Phase Lock Loop in [TOWNSEND et al, 1995] from theoretical and practical point of view. It reduces the maximum multipath delay that can cause a phase tracking error by a factor of 5 in practice, although this is 10 in theory. The maximum multipath phase error is unchanged.

Unfortunately, practical tests show severe difficulty in its ability to separate signals with short delays. Developments of MEDLL continue [CHAGGARA et al., 2002] and other recent techniques very similar as MEDLL exist, known under the names PEE (for Punctual Early Earliest - a Jet Propulsion Laboratory development) or Triple Carrier NCO (a Trimble patent). Refer to the literature collection [BRODIN, 2001]. The main problem of using multiple correlators occurs for short delay multipath, where algorithms fail to process the de-convolution of direct and reflected signals. Besides, the cost and technological problems raised in the GPS receivers architecture make the multipath estimation with multiple correlators not so competitive compared to the other phase mitigation technique presented hereafter.

Actually, an advanced phase correlation technique (inspired from the code MM correlators) is described in Leica's latest patent in the domain of multipath mitigation 
[STANSELL et al., 2000]. This was already introduced in another patent [STANSELL et al., 1996] and in [HATCH et al., 1997]. The Ashtech "Enhanced Strobe" correlator was developed in parallel. Both techniques are similar, and a detailed description is given in Chapter $5, \S 5.2$. They enable a significant step in phase multipath mitigation to be made, compared to the standard COSTAS phase correlator and even compared to MEDLL. They reduce the maximum multipath delay that can cause a phase tracking error by a factor of 10 to 40 (for the Leica Phase Multipath Mitigation Window), depending on the sampling rate of the signal. Only multipath with very small delays (those of a few metres) still cause large phase errors, and these errors have not been reduced in amplitude. The maximum multipath phase error is still unchanged.

To conclude the topic of phase multipath mitigation by correlation techniques, one can say that very close reflectors (causing additional path length of a few metres) still create multipath in phase measurements. The maximum multipath phase error is of the order of a few centimetres, with a maximum multipath delay that is around $7.5 \mathrm{~m}$. This last threshold corresponds to Leica PMMW technique with a $40 \mathrm{MHz}$ clock rate (see Chapter 5, § 5.2.4).

Lastly, [WEILL, 2003] demonstrates in simulation that an optimal combination of correlation processes applied on two signals, like GPS L2 and new L5, enable code and phase multipath errors to be reduced compared to their level if only one signal was used (despite each signal involves its own multipath parameters). [WEILL, 2003] offers one of the most advanced process, a priori designed to take advantage of any new GNSS signal. An improvement of almost an order of magnitude of code and phase errors is claimed. But this demonstration remains theoretical, since it is based on a new correlation process that is not standard in the industry, and still not implemented in real-time.

Note that the most advanced receiver correlation techniques, whether implemented or not, do not output measurements totally free of multipath errors, particularly for multipath with very short additional path lengths. As a consequence, multipath mitigation by data processing appears as a needed complement of receiver based mitigation. 


\section{3 - Multipath mitigation by data processing}

Many investigations have been - and are still - carried out in the field of multipath mitigation by data processing. Data processing means that the basic code and phase measurements have been taken (i.e. C/A and P(Y) codes, and L1 and L2 carrier phases observables), and, at the step of the calculation of the navigation solution, various strategies are used to mitigate or eliminate measurements that are affected by multipath. Two groups of methods can be identified, whether they use functional or stochastic modelling.

\subsection{1 - Functional modelling}

\subsubsection{1 - L1 and L2 phase combination to estimate multipath on code data}

Phase measurements on L1 and L2 enable code error to be estimated and removed, assuming that receiver noise and multipath error on phase are negligible compared to those on code (see [KEE and PARKINSON, 1994] and [EL-RABBANY, 1995]).

$\mathrm{C} 1=\rho+\mathrm{c}(\mathrm{dT}-\mathrm{dt})+\mathrm{Z}+\mathrm{I} 1+\mathrm{M}_{\mathrm{C} 1}+\varepsilon_{\mathrm{C} 1}$

$\lambda 1 \Phi 1=\rho+\mathrm{c}(\mathrm{dT}-\mathrm{dt})+\mathrm{Z}-\mathrm{I} 1+\mathrm{M}_{\Phi_{1}}+\varepsilon_{\Phi_{1}}+\lambda 1 \mathrm{~N}_{1}$

$\lambda 2 \Phi 2=\rho+\mathrm{c}(\mathrm{dT}-\mathrm{dt})+\mathrm{Z}-\mathrm{I} 2+\mathrm{M}_{\Phi_{2}}+\varepsilon_{\Phi_{2}}+\lambda 2 \mathrm{~N}_{2}$

where

$\mathrm{C} 1$ is the $\mathrm{C} / \mathrm{A}$-code measurement on $\mathrm{L} 1$

$\Phi 1$ and $\Phi 2$ are the phase measurements (in cycles) on L1 and L2 (with $\lambda 1$ and $\lambda 2$ wavelengths)

$\rho$ is the geometric distance between the receiver and satellite antennas

$\mathrm{dT}$ and $\mathrm{dt}$ are the clock offsets of the receiver and the satellite respectively

$\mathrm{N} 1$ and $\mathrm{N} 2$ are the ambiguities on $\Phi 1$ and $\Phi 2$ measurements

$\mathrm{Z}$ is the tropospheric range delay

I1 and I2 are the ionospheric range delays (depending on the frequency)

Mare the multipath range errors on $C 1, \Phi 1$ and $\Phi 2$ measurements, and $\varepsilon$ are the receiver noises on $C 1, \Phi 1$ and $\Phi 2$ measurements. 
By combining these equations, and by using the relation between ionospheric range delays and frequencies: $\mathrm{I} 2=(\mathrm{f} 1 / \mathrm{f} 2)^{2} \mathrm{I} 1$, the following expression for the multipath range error on $\mathrm{C} 1$ is obtained.

$\mathrm{C} 1-\left(1+2 /\left((\mathrm{f} 1 / \mathrm{f} 2)^{2}-1\right)\right) \Phi 1+\left(2 /\left((\mathrm{f} 1 / \mathrm{f} 2)^{2}-1\right)\right) \Phi 2=\mathrm{M}_{\mathrm{C} 1}+\varepsilon_{\mathrm{C} 1}+\mathrm{B}$

The range bias $\mathrm{B}$ is the ionospheric combination of the range bias due to the ambiguities:

$\mathrm{B}=\left(1+2 /\left((\mathrm{f} 1 / \mathrm{f} 2)^{2}-1\right)\right) \lambda 1 \mathrm{~N} 1-\left(2 /\left((\mathrm{f} 1 / \mathrm{f} 2)^{2}-1\right)\right) \lambda 2 \mathrm{~N} 2$

It can be estimated and removed from time series to produce the multipath range error on the code measurements. An estimation of $\mathrm{B}$ is the average value of the observables once combined in Eq. 2.4, since the average values of $\mathrm{M}_{\mathrm{Cl}}$ and $\varepsilon_{\mathrm{Cl}}$ are zero. The time series must be significantly longer in duration than the period of multipath.

The derivation of similar expressions for P-code measurements on L1 and L2 is also possible.

A practical use of this L1 and L2 phase combination can be found e.g. in [BISNATH et al., 1997] in the frame of GPS multipath assessment on an oil platform.

[BARNES, 2000] develops an analysis of this method on real data. Here are only recalled the conclusions:

- effects of multipath on code are effectively reduced (by $95 \%$ on the experimental $10 \mathrm{~m}$ baseline);

- the implementation in real-time requires a bias estimation and a careful attention to cycle slips;

- the fact that this combination of observables is applicable to an individual satellite and receiver combination makes it possible, in a calculation in double differences, to identify which pairs of satellite and receiver have the most significant multipath. 
2.3.1.2 - L1 and L2 ionospheric differential delay to estimate multipath on phase data

In [GEORGIADOU and KLEUSBERG, 1987], the combination of the direct signal and a single reflected signal is first computed. Then, the hypotheses that permit the consideration of the reflection in the frame of the geometric optics are made (see Chapter $1, \S 1.2 .1)$. This analysis shows cyclic variations in the multipath carrier phase error for both L1 and L2. These variations are characterised, and their frequencies are linked to that of the ionospheric delays experienced by L1 and L2. The method is summarised below.

The representation of the direct signal is that given in Chapter $1, \S 1.2 .4$. The environment is supposed to create a single multipath. So the direct and reflected signals are represented as follows:

$\mathrm{S}_{\text {direct }}=\mathrm{Sd}=\mathrm{A} \cos \Phi$

$\mathrm{S}_{\text {reflected }}=\mathrm{Sr}=\alpha \mathrm{A} \cos (\Phi+\Theta)$

where

A is the amplitude of the direct signal

$\Phi$ is the phase of the direct signal

$\alpha$ is the ratio of amplitude of the direct and reflected signals, and

$\Theta$ is the phase shift of the reflected signal.

Note that the code modulation is not represented there, and only the carrier phase appears. One can suppose that the code has been perfectly demodulated.

In Chapter $4, \S 4.1$ one gives a more comprehensive description of the parameters that relate to multipath in this representation $(\alpha$ and $\Theta)$.

These signals are combined in case of multipath, which means that they add together.

$S_{\text {combined }}=S c=S d+S r=\beta A \cos (\Phi+\Psi)$ 
where

$\beta=\left(1+2 \alpha \cos \Theta+\alpha^{2}\right)^{1 / 2}$, and

$\Psi=\arctan (\alpha \sin \Theta /(1+\alpha \cos \Theta))$.

Note: if $\alpha<<1$, which is generally true with selective antennas,

$\beta \sim 1+\alpha \cos \Theta$, and

$\Psi \sim \alpha \sin \Theta$.

So, for a given environment, the multipath carrier phase error $\Psi$ varies cyclically with $\Theta$ that itself varies with the satellite position through the additional path length $L$ travelled by the reflected wave (among other causes of phase variation, like reflection phase shift or antenna phase shift, see Chapter 1). Assuming that the reflection angle is the same as the incidence angle, it can be geometrically shown that:

$\Theta=2 \pi \mathrm{L} / \lambda=(4 \pi \mathrm{h} / \lambda) \sin (\theta)$

where

$\mathrm{L}$ is the additional path length

$h$ is the normal distance from the antenna phase centre to the reflective surface

$\theta$ is the angle of elevation of the satellite with respect to the surface, or grazing angle, and

$\lambda$ is the wavelength. 
Deriving Eq. 2.8 with time exhibits the frequency of the cyclic variation of $\Psi$ :

$\mathrm{f}_{\Psi}=(2 \mathrm{~h} / \lambda) \dot{\theta} \cos \theta$

where $\dot{\theta}$ is the rate of change in time of the elevation of the satellite with respect to the surface.

This frequency is:

-inversely proportional to the carrier wavelength $(\lambda)$;

- proportional to the distance between the antenna and the reflective surface (h);

- proportional to the cosine of the grazing angle $(\theta)$;

- proportional to the rate of change of this elevation $(\dot{\theta})$.

[GEORGIADOU and KLEUSBERG, 1987] suggests to use the first of the aforementioned properties. Hence, both the frequency of the multipath phase error and the carrier phase ionospheric delay are inversely proportional to the carrier wavelength. One can compute (by differencing Eq. 2.2 and 2.3) the difference $\Delta$ between the ionospheric delays experienced by L1 and L2, as follows:

$\Delta=\mathrm{I} 1-\mathrm{I} 2=-(\lambda 1 \Phi 1-\lambda 2 \Phi 2)+\left(\lambda 1 \mathrm{~N}_{1}-\lambda 2 \mathrm{~N}_{2}\right)+\left(\mathrm{M}_{\Phi_{1}}-\mathrm{M}_{\Phi_{2}}\right)$

The range, the clock terms and the tropospheric delay cancel. For these reasons, this combination is also called geometry free combination.

Eq. 2.11 can be differenced between a pair of L1/L2 receivers close to each other: the "differential ionospheric delay", denoted $\nabla \Delta$, is formed. In this particular case, the difference $\Delta$ between the ionospheric delays experienced by L1 and L2 is the same at both receivers. So the differential ionospheric delay, theoretically null for a short baseline, will be an indicator of multipath carrier phase errors at either or both receivers. This differential ionospheric delay is used to detect the multipath effects, satellite per satellite [GEORGIADOU and KLEUSBERG, 1987]. 
A way to check the performance of the proposed method is to compare its results with those of the function that depicts the theoretical multipath effects with the elevation of the satellite with respect to the surface and the rate of change of this elevation in a given environment. The comparison can be quite illuminating, despite the fact that multiple reflections are not considered in the model. It is mentioned that for this the L1 and L2 carrier phase centre variations need to be very well known.

The main drawback of the differential ionospheric delay is that it cannot separate the multipath effects on each carrier frequency. So, it is impossible to achieve a correction of the L1 (or L2) measurements only in this way.

\subsubsection{3 - Using the signal-to-noise ratio (SNR) as an observable of the carrier phase} multipath

Signal-to-noise ratio is measured and computed by receivers for each signal tracked in a channel (see Chapter 3, § 3.4). It can be considered as an observable.

[COMP and AXELRAD, 1996] presents an original method to identify multipath amplitude $(\alpha)$ and phase $(\Theta)$ characteristics relative to the direct signal, from which the error in the carrier phase can be reconstructed by the relation $\Psi \sim \alpha \sin \Theta$. The identification is based on the analysis of the variation of the signal-to-noise ratio (SNR) in the presence of multipath. SRN, like $\Psi$, varies cyclically with $\Theta$, but in quadrature. Actually, SNR directly depends on the amplitude of the combined signals: $\beta \sim 1+\alpha \cos \Theta$.

The identification is based on a combination of an adaptive notch filter in a first step and an optimisation least squares process in a second step, both running together. A 20 seconds interval decimation of phase and SNR data is used in static mode. This method of identification is capable to estimate relative amplitudes and phases for several reflected signals mixed in the received signal. A more detailed description of the method is given in Chapter 7, §7.3. 
The real multipath phase errors and those obtained by the identification and reconstruction processes are equal in magnitude, but their signs are ambiguous (the sign is not observable using SRN).

In differential positioning, differences of phases for a specific satellite will be contaminated by multipath phase errors at both base and rover antennas. For each satellite and for both base and rover, one can compute (by means of the identification and reconstruction processes) the magnitude of the error in the carrier phase due to multipath. All combinations in adding these errors or their opposites lead to a complete set of phase corrections to be tested.

As signs are unknown, the method implies that the total number of combinations that needs to be generated is $2^{n}$, where $n$ is the total number of modelled multipath errors. To solve the ambiguities (i.e. the signs of the errors), the method determines the combination that best fits the residuals of single differences of phases (for a satellite and a pair of antennas) in a navigation solution. Note that the single differences are relevant only if a common clock drives both receivers. In a more general case in a local network, double differences of phases need to be computed, and then the number $\mathrm{n}$ of combinations includes not only a given satellite but also the differencing one.

The complete processing is causal. Hence, it can be done in real-time. But a certain latency is necessary to make the resolution of the sign ambiguity, for instance in a window that moves along time.

The SRN based mitigation method has proved its efficiency in static positioning. The most serious limitations appear with data sets whose duration is less than 1 hour because of the time needed to obtain convergence of the sign identification.

Chapter 6 of [BARNES, 2000] is based on this mitigation technique. Improvement by $30 \%$ of the standard deviation of multipath corrupted phase measurements is claimed, in static mode, for additional path lengths over $10 \mathrm{~m}$. This technique is addressed further in the present document (in Chapter 7), where it is adapted and applied in kinematic mode. 


\subsubsection{4. - Using multiple antennas to remove multipath}

The idea is to measure phases in an array of antennas sufficiently closely spaced to exhibit the spatial correlation of multipath. The antennas are mounted in the same plane. Different universities and companies are currently involved in this research and this appears to be one of the most interesting and cost-effective solutions to date. [RAY, 1999] presents this technique. To simplify the notation, one will only write the equations for a pair of closely spaced antennas, using subscript 0 and 1 .

The relation $\Psi \sim \alpha \sin \Theta$ enables the computation of the carrier phase error when $\alpha$ and $\Theta$ have been estimated. $\alpha$ is supposed to be the same in both antennas (identical reflecting material and identical gain patterns), but $\Theta(\Theta 0$ and $\Theta 1)$, reflected signal phase delay, differs between antenna 0 and antenna 1 by the following relation:

$\Theta 1=\Theta 0+(2 \pi / \lambda) \mathrm{d}_{01} \cos \left(\mathrm{a}-\mathrm{a}_{01}\right) \operatorname{cose}$

where

$\lambda$ is the wavelength

$d_{01}$ is the distance between the antennas

$\mathrm{a}_{01}$ is the angle of azimuth of the vector from antenna 0 to antenna 1 , and

eand a are respectively the angles of elevation and azimuth of the reflected wave relatively to the plane of the antennas.

So $\alpha, \Theta 0$, e and a are the parameters to be estimated. The observable is the single difference of phase between antennas and a given satellite: $\Delta \Phi 01$, where integer ambiguities and differential range due to spatial separation of the antennas have been determined a priori, and the receivers clocks have been synchronised. The noise on the phase measurements is neglected. 
Only the differential multipath carrier phase error remains. Hence, the observable is: $\Delta \Phi 01=\Psi 1-\Psi 0$, which is a function, denoted $\mathrm{f}(\alpha, \Theta 0, \mathrm{e}, \mathrm{a})$, of $\alpha, \Theta 0$, eand a parameters.

The unknown parameters are estimated using an EKF (Extended Kalman Filter) and the sequence of observables. $\delta \mathrm{f} / \delta \alpha, \delta \mathrm{f} / \delta \Theta 0, \delta \mathrm{f} / \delta \mathrm{e}, \delta \mathrm{f} / \delta \mathrm{a}$ are computed to form the design matrix of the filter, and so linearise the problem.

Such a method can be extended to other observables, including for instance code measurements and signal-to-noise ratio [RAY et al., 1999]. Amongst other advantages, this method is generic, since it uses the formalism of Kalman. As a consequence, it is also applicable to systems with other observables and other unknown parameters. Code and SNR data output by the receivers, in addition to phase data, can be used together.

Hence, an additional observable is the single difference of code: $\Delta \mathrm{P} 01$. It is shown in [RAY et al., 1999] that $\Delta \mathrm{P} 01=\tau 1-\tau 0$ is a function of $\alpha, \alpha^{\prime}, \Theta 0$, eand a parameters, where $\tau$ denotes the code multipath error and $\alpha^{\prime}$ - an additional unknown parameter - the correlation ratio in the code loop (see Chapter 3). Furthermore, the ratio of the variation of the signal-to-noise ratio in the antennas is again a function of the same parameters.

The last developments [RAY, 2000] achieved $15 \%$ average reduction of the multipath carrier phase error (and up to $50 \%$ for certain satellites), with additional path lengths of the order of $10 \mathrm{~m}$. More recently [FARRET and SANTOS, 2001] tried a simplification of the method with only two antennas, and obtained quite similar results. Note that very small additional path lengths are not particularly addressed in these works.

\subsection{2 - Stochastic modelling}

\subsubsection{1 - Thermal noise in carrier phase tracking loop}

Stochastic modelling is based on the formula that links the standard deviation $\sigma_{\mathrm{PLL}}$ of carrier phase measurements to several parameters of the channels: 
$\sigma_{\mathrm{PLL}}=\sqrt{\frac{\mathrm{BW}}{\mathrm{c} / \mathrm{n} 0}\left[1+\frac{1}{2 \mathrm{Tc} / \mathrm{n} 0}\right]} \lambda / 2 \pi$

where

BW is the phase loop bandwidth (in $\mathrm{Hz}$ )

$\mathrm{c} / \mathrm{n} 0$ is the ratio of GPS signal amplitude and noise amplitude in a $1 \mathrm{~Hz}$ bandwidth; if $\mathrm{C} / \mathrm{NO}$ is given in $\mathrm{dB}-\mathrm{Hz}$, it must be transformed into a ratio by applying the relation:

$\mathrm{c} / \mathrm{n} 0$ (ratio) $=10^{\mathrm{C} / \mathrm{N} 0(\text { in dB-Hz) } / 10}$

$\mathrm{T}$ is the phase loop integration period (in s).

This formula is available for a standard Phase Lock Loop (see Chapter 3, §3.3). It assumes that the thermal noise is the principal cause of the receiver noise $\varepsilon_{\Phi}$ that effects the phase measurements (see Eq. 2.2 and 2.3).

Many reference books about GPS explain in more detail the noise sources and their quantification [WARD, 1996]. The synthesis in [LANGLEY, 1997] is also of interest. But, in the frame of the research related to stochastic modelling, only the most important source of noise (thermal noise) has generally been considered.

In usual conditions of signal reception, Eq. 2.13 is well approximated by:

$\sigma_{\mathrm{PLL}}=\sqrt{\frac{\mathrm{BW}}{\mathrm{c} / \mathrm{n} 0}} \lambda / 2 \pi$

since typical values of integration period are of the order of a few milliseconds, and a few tenths of $\mathrm{dB}-\mathrm{Hz}$ for $\mathrm{C} / \mathrm{N} 0$.

In the GPS positioning process, least squares resolution needs variances of observables, which can be estimated from Eq. 2.15 and c/n0 measurements in receivers. Note that $\mathrm{c} / \mathrm{n} 0$ is another expression of signal-to-noise ratio that is output by each channel 
for each satellite signal tracked. A relation of proportionality between c/n0 and SNR (ratio) is a commonly used approximation, with a coefficient equal to the tracking bandwidth:

$\mathrm{SNR}($ ratio $)=(1 / \mathrm{BW}) \mathrm{c} / \mathrm{n} 0$ (ratio)

Eq. 2.16 means that $\mathrm{c} / \mathrm{n} 0$ quantifies the amplitude of the GPS signal input to the receiver, whereas SNR quantifies the amplitude of the signal effectively used in the tracking process, thus involving its bandwidth (both amplitudes being related to that of the noise).

\subsubsection{2 - SIGMA-E model}

The idea is to use Eq. 2.14 between variance of phase measurements and signal-tonoise ratio in order to weight the phase measurements that are combined in least squares processing. For instance, supposing a $2 \mathrm{~Hz}$ bandwidth tracking loop, and a direct GPS signal with $\mathrm{C} / \mathrm{N} 0$ of $45 \mathrm{~dB}-\mathrm{Hz}$, Eq. 2.14 gives $\sigma_{\mathrm{PLL}}=0.2 \mathrm{~mm}$ (see Fig. 2.2). If the signal is attenuated by phenomena like reflection or diffraction, $\mathrm{C} / \mathrm{N} 0$ may decrease in this case to around $30 \mathrm{~dB}-\mathrm{Hz}$ (under which most receivers would not track anymore), and then Eq. 2.14 gives $\sigma_{\mathrm{PLL}}=1.3 \mathrm{~mm}$.

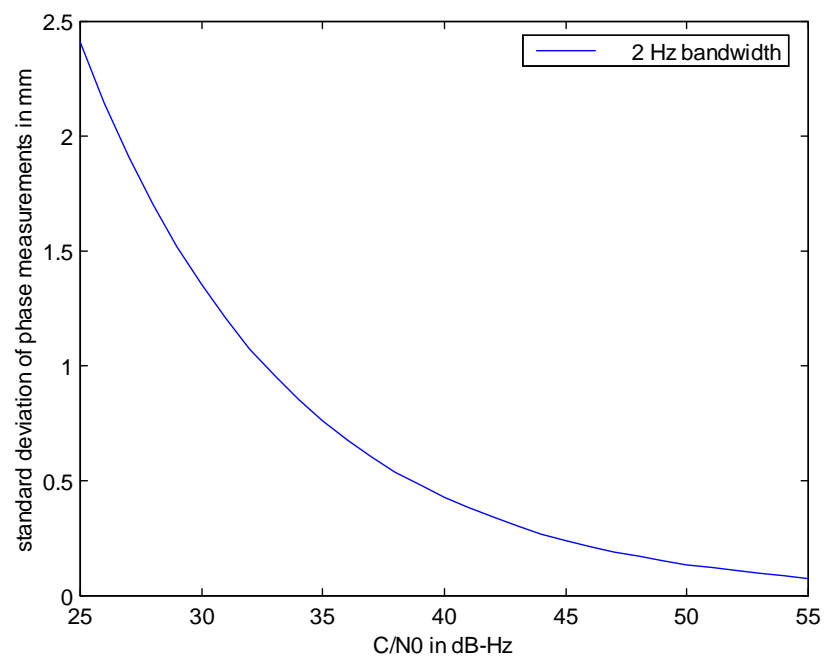

Figure 2.2: standard deviation of phase measurements in function of $\mathrm{C} / \mathrm{N} 0$ 
The main objective of SIGMA- $\varepsilon$ model is to enable the use of observations affected by diffraction at low elevation angles above the horizon, and to a certain extent any observations perturbed by multipath. A cut-off angle at e.g. $10^{\circ}$ would suppress observations however useful, and would make the satellite geometry poorer and the attainable precision decreased. This model classically enables the cut-off angle to be set up at about $5^{\circ}$.

SIGMA- $\varepsilon$ model is the original stochastic model produced by Technical University of GRAZ [HARTINGER and BRUNNER, 1999]. It assigns epoch by epoch weights (based on signal-to-noise ratio) to the double differenced data, by using propagation law of variances on individual receiver and satellite data.

$\mathrm{Q}_{\mathrm{PLL}}=\sigma_{\mathrm{PLL}}^{2}=\left(\mathrm{BW}^{*} \lambda^{2} / 4 \pi^{2}\right) * 10^{-\mathrm{C} / \mathrm{NO}(\text { in } \mathrm{dB}-\mathrm{Hz}) / 10}$

The model does not downweight DD phase data like an elevation angle model would do (e.g. $\left.1 / \sin ^{2}(\mathrm{el})\right)$, and therefore seems to achieve a good balance in the tradeoff between the actual level of noise in data and the satellite geometry. It is better than a simple elevation function because it is based on the effective signal quality measured by each channel.

\subsubsection{3 - SIGMA- $\Delta$ model}

SIGMA- $\Delta$ model contains an additional parameter to SIGMA- $\varepsilon$ model [BRUNNER et al., 1999]. This parameter is the absolute value of the deviation $\Delta$ to the expected SNR value. It is actually possible to know a priori by calibration what value of signal-to-noise ratio is expected from a satellite at a given elevation (see $§ 2.2 .1$ ).

In an environment where signals are perturbed, the deviation $\Delta$ to the expected SNR is computed and introduced into the weighting model using the following formula:

$\mathrm{Q}_{\mathrm{PLL}}=\sigma_{\mathrm{PLL}}^{2}=\left(\mathrm{BW}^{*} \lambda^{2} / 4 \pi^{2}\right) * 10^{-(\mathrm{C} / \mathrm{N} 0 / 10-\alpha|\Delta|)}$ 
where $\alpha$ is empirical and equals 2 .

The efficiency of SIGMA- $\Delta$ model to cope with signals contamination by multipath is

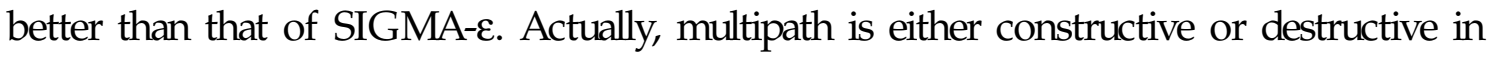
terms of combined signals. SIGMA- $\varepsilon$ downweights essentially a destructive combination and the contrary for a constructive one, whereas both cases of interference deviate from the expected SNR value and should be treated similarly. This is done in SIGMA- $\Delta$.

Lastly, the implementation of this model in real-time needs a self-calibration: that is to say the estimation of a "template function" of signal-to-noise ratio versus elevation of satellites [RICHTER and EULER, 2001].

\subsubsection{4 - Extended weight model for GPS phase observations}

This section reports complementary developments published by Technical University of GRAZ [WIESER and BRUNNER, 2000] to SIGMA- $\varepsilon$ and SIGMA- $\Delta$ modelling.

Experimental results of both models conclude that weighting exclusively on signal-tonoise ratio and its deviation to a "template function" of elevation does not fit with the actual quadrature between the multipath phase error and the SNR (when $|\Delta|$ is minimum, the phase error is generally maximum, and reciprocally). Furthermore, diffraction is not optimally modelled with SIGMA- $\varepsilon$ and SIGMA- $\Delta$.

A robust estimation is proposed. The residuals on DD of phase measurements in an epoch by epoch positioning process are used as an input of an extended weight model, whose main features are described below.

The standard Gauss-Markov system of equations relating the unknown positioning vector $(x)$ and the vector of observations $(y)$ - that are here the DD of phase measurements at a given epoch - is formulated:

$y=A^{*} x+r$ 
where

$A$ is the Jacobian matrix, and

$r$ is the vector of residuals on DD of phase measurements.

$r$ are supposed to be normally distributed, with a zero mean value, and an initial variance matrix Qyy computed by the SIGMA- $\varepsilon$ model and a known a priori variance factor $\sigma_{0}^{2}$ :

$\mathrm{r} \sim \mathrm{N}\left(0, \sigma_{0}^{2} \mathrm{Qyy}\right)$

The inversion of the system gives an estimation of the residuals:

$r=\left(A\left(A^{T} Q y y^{-1} A\right)^{-1} A^{T} Q y y^{-1}-I\right) y$

and their variance matrix:

Qrr $=$ Qyy- A $\left(A^{\mathrm{T}} \mathrm{Qyy}^{-1} \mathrm{~A}\right)^{-1} \mathrm{~A}^{\mathrm{T}}$

For each observation (each pair of receivers and pair of satellites), denoted with index " $i$ ", the individual residual $r_{i}$ is compared to the corresponding $3 \sigma_{i}$ envelope, with:

$\sigma_{\mathrm{i}}^{2}=\sigma_{0}^{2} \mathrm{Qrr}(\mathrm{i}, \mathrm{i})$

The weight matrix $\mathrm{Qyy}^{-1}$ used in the next epoch system is a function of the comparison made before, between the individual residuals $r_{i}$ and their corresponding $3 \sigma_{i}$ envelope.

For residuals out of this envelope, the corresponding term in the next weight matrix is multiplied by a down-weighting factor $\mathrm{f}_{\mathrm{i}}$ :

$$
\mathrm{f}_{\mathrm{i}}=\exp \left(-|\mathrm{ri}| / 3 \sigma_{\mathrm{i}}\right)
$$

For residuals within this envelope, the corresponding term in the next weight matrix is unchanged from the current epoch to the next $\left(f_{i}=1\right)$. 
[WIESER and BRUNNER, 2000] shows improvement of positioning by up to a few centimetres particularly in cases of diffraction and multipath errors, that SIGMA- $\varepsilon$ and SIGMA- $\Delta$ failed to mitigate properly. This efficiency is due to the weight strategy, which is based on the actual residuals. Note only limited data sets are given to illustrate the method.

Last but not least the method works epoch by epoch, which makes it equally suitable for kinematic and static applications.

\section{$2.4-$ Conclusions}

Contrary to antenna or receiver mitigation design, and also contrary to functional modelling, the stochastic methods do not directly improve the individual ranges. The comparison between these methods and those modifying the ranges (i.e. the "raw" measurements) is only possible on the final positioning solution, which tends to smooth the differences between the compared techniques. Furthermore, the different technologies listed in this chapter have never been compared on a common basis of experimental data. So, no objective conclusion can be drawn in terms of comparison of performances.

Despite the fact that very significant improvements have been made in multipath mitigation by the use of specifically designed antennas and correlators in receivers, multipath effects still remain in both code and phase measurements.

\section{Code measurements:}

The most advanced code correlators still produce errors in measurements in the presence of multipath. These errors are bounded, as shown in Chapters 3 and 4, but remain significant: for C/A-code, their level is a few metres; for P-code, a few decimetres. An effective method (presented in § 2.3.1.1) combining code observations and dual frequency phase data enables code multipath to be significantly reduced.

Concerning the research carried out in the frame of this thesis that focuses on phase measurements, the examination of code data can be useful since it provides us with information of the occurrence of reflections that are likely to affect also the phase data. 
Furthermore, as already used by [RAY et al., 1999], the relation between code error and phase error in case of multipath is also to be considered in functional modelling approach of multipath mitigation.

Phase measurements:

The research domain in which this work takes place concerns the mitigation of phase measurements in a real-time kinematic GPS processing. No a priori knowledge of multipath occurrence is available. Hence, a first level of the problem of multipath mitigation is the detection of the presence of multipath in the observables.

The ionospheric differential delay (also called geometry free combination) is an observable of multipath on short baselines presented in [GEORGIADOU and KLEUSBERG, 1987]. It enables the presence of multipath to be identified. However, the inverse problem that consists in separating the multipath phase errors on L1 and L2 carrier phases has no solution. A suggestion is to use the ionospheric differential delay as a final test to assess the effectiveness of corrections applied to phase measurements obtained for instance from a method based on the signal-to-noise ratio (SNR).

The use of SNR is of great interest [COMP and AXELRAD, 1996]; [BARNES, 2000]. The issue of assigning the sign of the corrections to be applied on phase measurements remains crucial. This problem will be addressed in this thesis, and investigations will be carried out on the possibility of improving this method by means of phase measurements output by the new Multipath Mitigation Windows correlator presented in Chapter 4.

Concerning the multiple mobile antennas combined to remove multipath, the concepts used are in the domain of this research, but one still seeks a solution with only one rover antenna, as this is more likely to be practically useful.

Lastly, this research will focus on the functional modelling approach for phase multipath mitigation. A wide variety of new processes is possible to be developed with the new observables provided by the MMW correlator. This work also aims at filling a gap in the current capabilities of multipath mitigation techniques in case of very close reflectors. 
Chapter 3

\section{GPS SIGNAL PHASE AND CODE TRACKING IN A STANDARD RECEIVER}

This chapter gives a brief description of the GPS signal structure. Afterward, the main steps of the signal processing carried out in a standard receiver are described: signal downconversion, signal sampling, and phase and code tracking.

The main references for writing this chapter are [WARD, 1996], [BRAASCH, 1996], [VAN NEE, 1995] and [RAY et al., 1999].

\section{1 - Description of the GPS signal structure}

A simplified description of the GPS signal structure is displayed in Fig. 3.1.

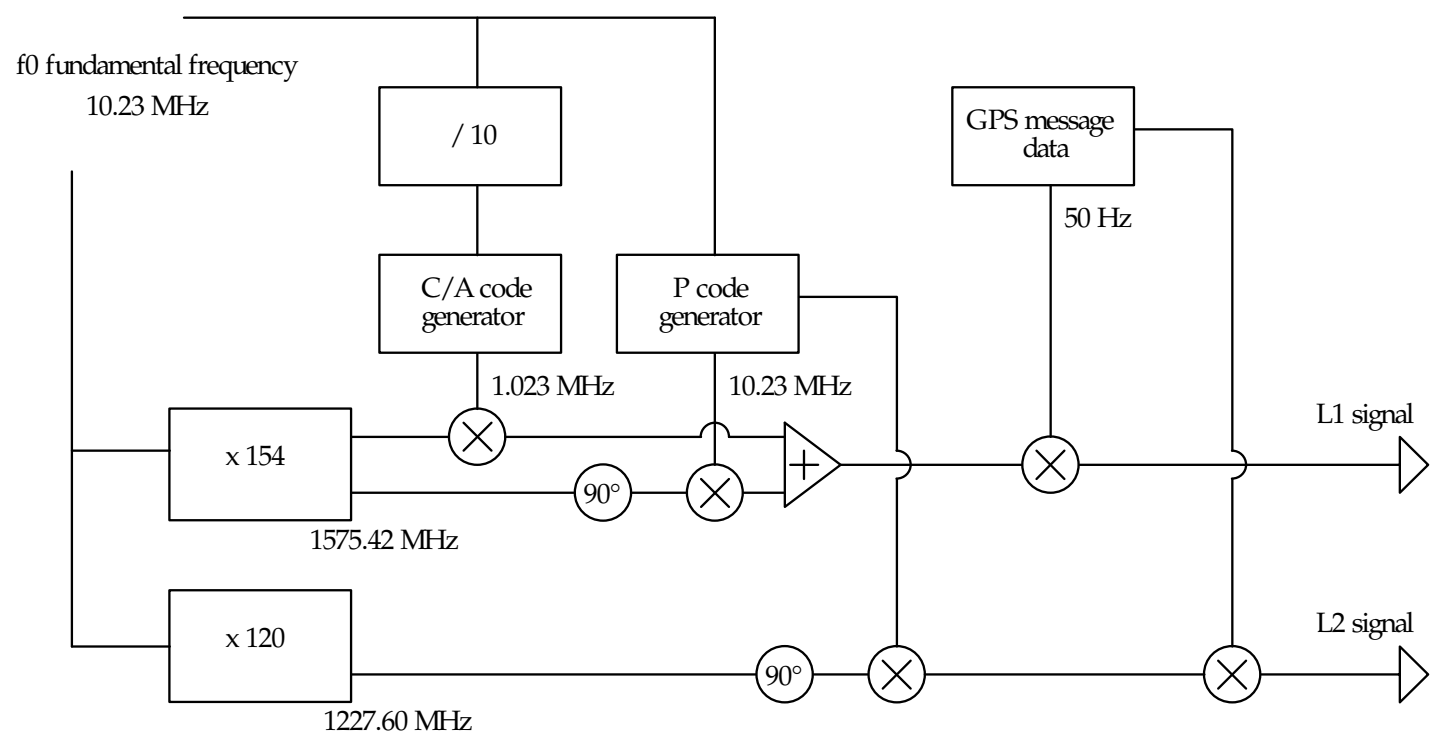

Figure 3.1: simplified GPS signal structure 
Two carrier waves (at the approximate frequencies L1: $1575.42 \mathrm{MHz}$ and L2: 1227.60 MHz) are modulated by pseudo-random noise codes (C/A-Gold-code and Pcode). For defence purpose, the P-code is encrypted into the Y-code, and its access is limited to authorised users. Besides, the carrier waves are modulated by the GPS message data.

The GPS signal use a spread-spectrum technique by modulating the two carrier waves. The GPS PRN coding consists in a phase modulation as illustrated in Fig. 3.2: it works by changing the phase by $180^{\circ}$ at every code alternation.

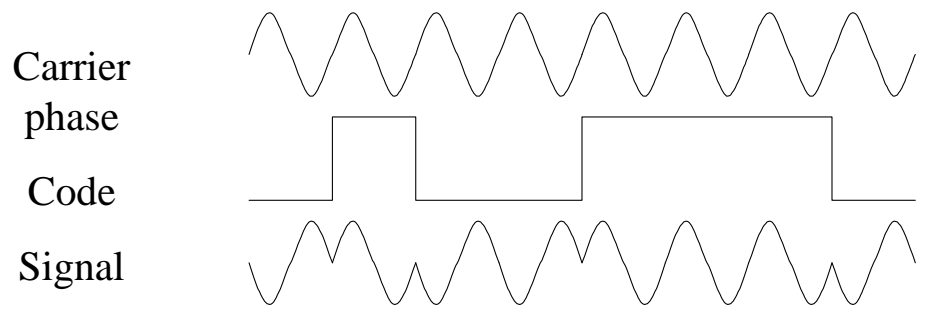

Figure 3.2: code carrier phase modulation

All techniques of code acquisition and tracking in GPS receivers basically use the main properties of the Gold codes. They are pseudo-random noise codes, which means that their autocorrelation function is an approximation of a Dirac (see Fig. 3.3, where a 15 bit PRN binary sequence is displayed, as an example, along with its autocorrelation function). And the cross-correlation between different PRN codes (corresponding to different satellites) is "near zero". These properties base the technique of spread-spectrum Code Division Multiple Access (CDMA): an individual satellite can be identified by its own PRN code and different satellites do not interfere since PRN codes cross-correlation is null. 


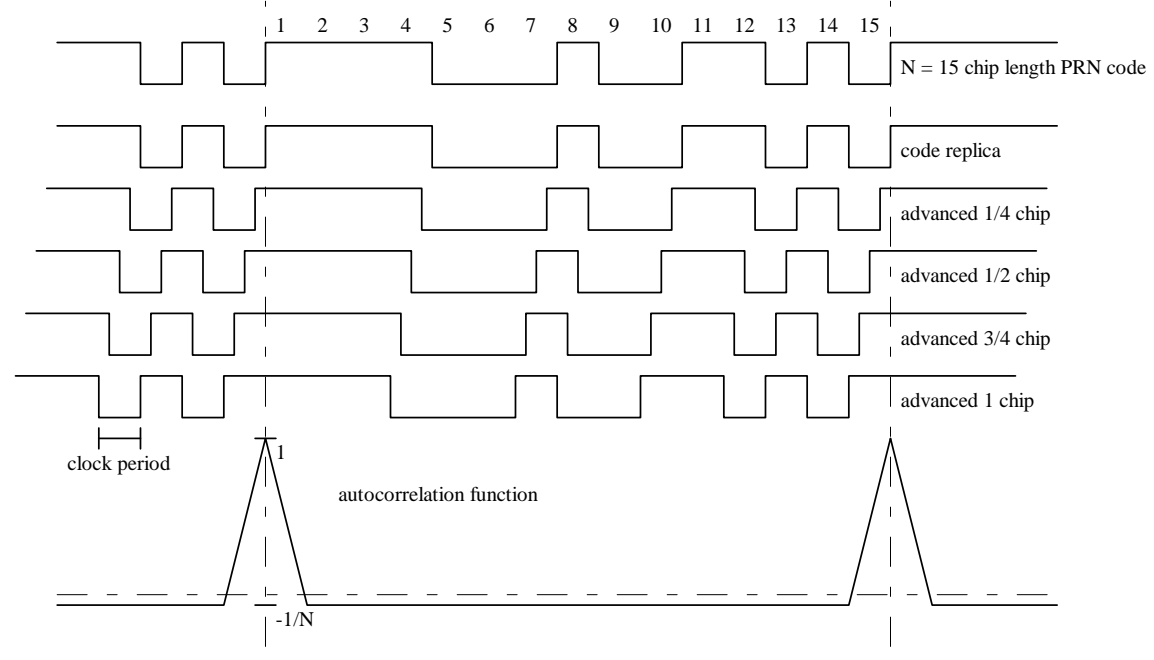

Figure 3.3: PRN code autocorrelation function

Lastly, it is important to mention that the GPS signal is right-hand circularly polarised (RHCP). The GPS antennas are designed to amplify the RHCP input signal, and attenuate the opposite LHCP.

In this dissertation, a complete description of the GPS signal is not necessary. The analyses given further are based on a single frequency. L1 (except if L2 is explicitly mentioned). A single code (in addition to the message data) is considered. The concepts that are presented can be generalised to both codes and both frequencies.

\section{2 - Signal down-conversion and sampling}

\subsection{1 - Signal down-conversion}

Modern receivers use digital signal processing, after the antenna filtering and the signal amplifying. The last analogical step in the receivers is a down-conversion of the GPS signal, from the "radio frequency" (or RF) original signal, down to the so-called "intermediate frequency" (or IF) signal.

Amongst several possible techniques, the IF signal can be derived from the RF signal by the help of mixing with a generated signal. The frequency of the generated signal is 
chosen with respect of the frequency of the original signal, and that required as IF. The receiver local oscillator pilots the generation of the signal to be mixed.

It is important to notice that the code, phase and Doppler of the original signal is preserved by the down-conversion. The down-conversion only reduces the frequency $f$ of the original signal, but it does not affect the code, neither the phase nor the Doppler variation of this frequency around its nominal value $\mathrm{fn}$.

The Doppler affected frequency fd is:

$\mathrm{fd}=\mathrm{fn}\left(1-\frac{\dot{\rho}}{\mathrm{C}}\right)$

where

$\mathrm{fn}$ is the nominal frequency of the carrier wave

$c$ is the speed of light, and

$\dot{\rho}$ is the geometric range rate between the satellite and receiver antennas.

Suppose that original received signal is:

$\mathrm{S}=\mathrm{C}(\mathrm{t}) * \sin \left(2 \pi^{*} \mathrm{fd} * \mathrm{t}+\varphi_{0}\right)=\mathrm{C}(\mathrm{t}) * \sin \left(2 \pi^{*} \mathrm{fn} * \mathrm{t}-2 \pi^{*} \mathrm{fn}{ }^{*} \frac{\dot{\rho}}{\mathrm{C}} * \mathrm{t}+\varphi_{0}\right)$

where

$\mathrm{fd}$ is the Doppler affected frequency for a given satellite

$\mathrm{C}$ is the received PRN code time series of this satellite, and

$\varphi_{0}$ is the phase of the received signal at $t=0$ that can be set to zero conventionally.

The term: $2 \pi^{*} \mathrm{fn}^{*} \frac{\dot{\rho}}{\mathrm{c}} * \mathrm{t}$ is the phase shift due to the Doppler.

The signal for down-conversion is supposed to be generated at a constant specified frequency: 
$S_{\text {generated }}=\sin \left(2 \pi^{*} f s^{*} t\right)$

where

fs is the specified frequency used for down-conversion.

The down-conversion is a product of the signals $S$ and $S_{\text {generated: }}$

$$
\begin{aligned}
& S_{\text {down-converted }}=S^{*} S_{\text {generated }}=C(t) * \sin \left(2 \pi^{*} \mathrm{fd}^{*} \mathrm{t}+\varphi_{0}\right)^{*} \sin \left(2 \pi^{*} \mathrm{fs}^{*} \mathrm{t}\right) \\
& S_{\text {down-converted }}=C(\mathrm{t}) *\left[\cos \left(2 \pi^{*}(\mathrm{fd}-\mathrm{fs})^{*} \mathrm{t}+\varphi_{0}\right)-\cos \left(2 \pi^{*}(\mathrm{fd}+\mathrm{fs})^{*} \mathrm{t}+\varphi_{0}\right)\right] / 2
\end{aligned}
$$

In Eq. 3.5, the ( $\mathrm{fd}+\mathrm{fs})$ high frequency term can be eliminated in a low-pass filter and, as a consequence, only the (fd-fs) low frequency term remains.

Hence, the received signal after down-conversion and filtering is:

$$
\begin{aligned}
& S_{\text {down-converted and filtered }}=1 / 2 * C(t) * \cos \left(2 \pi^{*}(\mathrm{fd}-\mathrm{fs}) * t+\varphi_{0}\right) \\
& S_{\text {down-converted and filtered }}=1 / 2 * C(t) * \cos \left(2 \pi^{*}(\mathrm{fn}-\mathrm{fs})^{*} \mathrm{t}-2 \pi^{*} \mathrm{fn}^{*} \frac{\dot{\rho}}{\mathrm{c}} * \mathrm{t}+\varphi_{0}\right)
\end{aligned}
$$

The phase term due to the Doppler $\left(2 \pi^{*} \mathrm{fn}^{*} \frac{\dot{\rho}}{\mathrm{c}} * \mathrm{t}\right)$ is preserved, as well as the phase and the code. The intermediate frequency (fi) is the difference (fn-fs) between the nominal frequency and that of the generated signal.

To conclude, the signal at IF - like the RF original signal - is modulated by the code (and the message data), and keeps its phase and Doppler properties.

In receiver technology and design, it is common to display the frequency in F-units, where $\mathrm{F}$ is $5.115 \mathrm{MHz}$. Hence, L1: $1575.42 \mathrm{MHz}$ is $308 \mathrm{~F}$ (L1 frequency is $154 * 10.23 \mathrm{MHz}$ ) and L2: $1227.60 \mathrm{MHz}$ is $240 \mathrm{~F}$ (L2 frequency is $120 * 10.23 \mathrm{MHz}$ ). The Leica System 500 Measurement Engine (ME) uses the same down-conversion for L1 and L2, with a generated signal at 270F, leaving (as IF) 38F for L1 and 30F for L2. 


\subsection{2 - Signal sampling}

After the down-conversion, the signal at IF is sampled.

In the Leica ME, the sampling rate is $8 \mathrm{~F}$ (approximately $40 \mathrm{MHz}$ ). The L1 $38 \mathrm{~F}$ signal sampled at $8 \mathrm{~F}$ leaves $-2 \mathrm{~F}$, as shown in Fig. 3.4, as well as the $\mathrm{L} 230 \mathrm{~F}$ signal. The frequency of the apparent carrier is $2 \mathrm{~F}$.

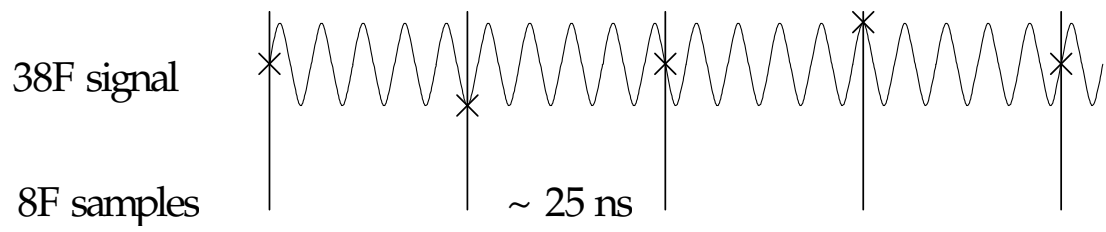

Figure 3.4: L138F signal sampling in Leica ME

Note: certain receivers do not make signal mixing for down-conversion, but directly sample the RF original signal at a frequency well chosen. Typically, other Leica receivers sample the signal at a frequency of $8.5 \mathrm{~F}$. The frequency of the apparent carrier of the sampled signals will be $2 \mathrm{~F}$ for both L1 and L2.

At this stage of the process, the received signal has been down-converted, filtered and sampled in order to be processed further. It is a mixture of the different GPS signals emitted by the visible satellites. Each has its own Doppler and specific identifying PRN code. In the receiver, multiple channels will carry out the acquisition and tracking of the different signals that are mixed. Each channel provides signal code and phase tracking, and the corresponding measurements in dedicated loops.

Note: in the following sections that deal with phase and code tracking, the received signal is implicitly down-converted, filtered and sampled. 


\section{3 - Phase tracking}

This section presents the main features of a standard phase lock loop (PLL), also called COSTAS loop, which achieves phase tracking and phase measurements in standard receivers. The functioning of a PLL is detailed step by step.

Suppose signal tracking in steady state. This means that the initialisation of the acquisition process is supposed to be done, and that keep tracking is the only problem.

In accordance with this hypothesis, both $\tau$, which denotes the instantaneous code deviation (i.e. the code error of the code tracking loop), and $\varphi$, which denotes the instantaneous phase deviation (i.e. the phase error of the phase tracking loop), are close to zero.

The term "deviation" refers to the signal either in terms of code synchronisation between the local receiver code generator and the code effectively received, or in terms of phase synchronisation, between the local receiver phase generator and the phase effectively received.

The time when the steady state is achieved is denoted $\mathrm{t}_{0}$.

The process of phase measurements is based on ASIC hardware counting of the phase adjustments that are measured and applied iteration by iteration in a controlled lock loop. In each channel, a given PRN replica is kept in both phase and code synchronisation (see §3.4) with its corresponding component in the received signal mixture. There are as many replicas as PRN codes to be demodulated in the received signal, within the technological limitations of the GPS unit (i.e. the maximum number of channels).

The replica is generated by means of a Numerically Controlled Oscillator (NCO), where the carrier frequency is controlled in order to fit as well as possible that of the received signal (converted to the intermediate frequency). The control of the frequency is possible through a loop filter in which the phase deviation is input and corresponds to the incremental phase adjustment to be applied until the next iteration of the loop. This phase 
deviation is processed in a correlator that is detailed further in this section. It enables the phase loop to be locked, by adjusting the carrier frequency of the replica.

A mathematical explanation of the functioning of the phase correlator is given below.

The received signal is denoted as follows:

$S(t)=A D(t) * C(t) * \sin \left(2 \pi^{*} f^{*}\left(t-t_{0}\right)+\varphi_{0}\right)$

where:

$A$ is the amplitude of the received signal

$\mathrm{D}$ is the data bit corresponding to the GPS message

$\mathrm{f}$ is the Doppler affected intermediate frequency, and

$\varphi_{0}$ is the phase of the received signal at $t_{0}$.

Compared to Eq. 3.7, the $1 / 2$ factor has been included in the amplitude factor A. The cosine has been replaced by a sine to keep in accordance with the most usual notations. This is equivalent to changing the phase $\varphi_{0}$ of the received signal at the instant $t_{0}$. In the representation of the received signal in Eq. 3.8, $\varphi_{0}$ can be set to zero conventionally.

Note that $\mathrm{f}$ should be denoted $\mathrm{f}(\mathrm{t})$ as it is not constant but time varying through $\dot{\rho}$ :

$\mathrm{f}(\mathrm{t})=(\mathrm{fn}-\mathrm{fs})-\mathrm{fn}^{*} \frac{\dot{\rho}}{\mathrm{c}}=\mathrm{fi}-\mathrm{fn}^{*} \frac{\dot{\rho}}{\mathrm{c}}$

The aim of the phase tracking loop is to keep in phase with the received signal, despite the Doppler variation in time mainly, and also despite the possible phase shift of the NCO before the receiver gets stable in temperature. To a lesser extent, the propagation effects (that cause the "phase velocity" to change) have a certain variation in time because the wave makes its way through the atmosphere differently from an instant to another. 
Concerning the Doppler, suppose that the range rate $\dot{\rho}$ equals $\dot{\rho}_{0}$ at the instant $\mathrm{t}_{0}$ when the steady state is achieved. Hence, at the instant $t_{0}$ the replica is generated at the frequency:

$$
\mathrm{f}_{0}=\mathrm{fi}-\mathrm{fn}^{*} \frac{\dot{\rho}_{0}}{\mathrm{C}}
$$

The phase of the replica is $\left(2 \pi^{*} f_{0}^{*}\left(t-t_{0}\right)\right)$, while that of the received signal is $\left(2 \pi^{*} f(t)^{*}(t-\right.$ $\left.\mathrm{t}_{0}\right)$ ). So, the phase deviation between the received signal and the replica equals:

$$
\varphi=2 \pi^{*}\left(\mathrm{f}(\mathrm{t})-\mathrm{f}_{0}\right)^{*}\left(\mathrm{t}-\mathrm{t}_{0}\right)
$$

This phase deviation can be obtained electronically in a correlation process. The received signal is correlated with two generated replicas for every channel, each channel being dedicated to a specific satellite (i.e. a specific PRN code). The replicas are:

- the punctual code in phase: $\operatorname{IP}(\mathrm{t})=\mathrm{C}(\mathrm{t}+\tau) * \sin \left(2 \pi^{*} \mathrm{f}_{0}^{*}\left(\mathrm{t}-\mathrm{t}_{0}\right)\right)$

- the punctual code in quadrature: $\mathrm{QP}(\mathrm{t})=\mathrm{C}(\mathrm{t}+\tau) * \cos \left(2 \pi^{*} \mathrm{f}_{0}^{*}\left(\mathrm{t}-\mathrm{t}_{0}\right)\right)$

where

$\tau$ is the code error of the code tracking loop.

The operations of correlation are:

$$
\begin{aligned}
& \mathrm{S}^{*} \mathrm{IP}(\mathrm{t})=\mathrm{AD}(\mathrm{t})^{*} \sin \left(2 \pi^{*} \mathrm{f}(\mathrm{t})^{*}\left(\mathrm{t}-\mathrm{t}_{0}\right)\right)^{*} \sin \left(2 \pi^{*} \mathrm{f}_{0}^{*}\left(\mathrm{t}-\mathrm{t}_{0}\right)\right) * \mathrm{C}(\mathrm{t})^{*} \mathrm{C}(\mathrm{t}+\tau) \\
& \quad=\mathrm{AD}(\mathrm{t}) *\left(\cos \left(2 \pi^{*}\left(\mathrm{f}(\mathrm{t})-\mathrm{f}_{0}\right)^{*}\left(\mathrm{t}-\mathrm{t}_{0}\right)\right)-\cos \left(2 \pi^{*}\left(\mathrm{f}(\mathrm{t})+\mathrm{f}_{0}\right)^{*}\left(\mathrm{t}-\mathrm{t}_{0}\right)\right)\right) / 2 * \mathrm{R}(\tau) \\
& \mathrm{S}^{*} \mathrm{QP}(\mathrm{t})=\mathrm{AD}(\mathrm{t})^{*} \sin \left(2 \pi^{*} \mathrm{f}(\mathrm{t})^{*}\left(\mathrm{t}-\mathrm{t}_{0}\right)\right)^{*} \cos \left(2 \pi^{*} \mathrm{f}_{0}^{*}\left(\mathrm{t}-\mathrm{t}_{0}\right)\right)^{*} \mathrm{C}(\mathrm{t})^{*} \mathrm{C}(\mathrm{t}+\tau) \\
& \quad=\mathrm{AD}(\mathrm{t})^{*}\left(\sin \left(2 \pi^{*}\left(\mathrm{f}(\mathrm{t})+\mathrm{f}_{0}\right)^{*}\left(\mathrm{t}-\mathrm{t}_{0}\right)\right)-\sin \left(2 \pi^{*}\left(\mathrm{f}(\mathrm{t})-\mathrm{f}_{0}\right)^{*}\left(\mathrm{t}-\mathrm{t}_{0}\right)\right)\right) / 2 * \mathrm{R}(\tau)
\end{aligned}
$$

where 
$\mathrm{R}$ is the code autocorrelation function. Since $\tau$ equals zero when the code loop is locked, $R(\tau)$ equals 1 .

In the ASIC, the sum frequency term $\left(f(t)+f_{0}\right)$ that is approximately twice the intermediate frequency can be low-pass filtered. The difference frequency term $\left(\mathrm{f}(\mathrm{t})-\mathrm{f}_{0}\right)$ corresponds to the Doppler variation between $t$ and $t_{0}$, and leads to the phase error $\varphi$ of the phase tracking loop.

The low-pass filter is mathematically an integration and averaging operation. The integrated components corresponding to $\left(f(t)+f_{0}\right)$ are null. Those corresponding to $\left(f(t)-f_{0}\right)$ are given in the following two equations:

$$
\begin{aligned}
& I={ }_{\text {PIT }} \int S^{*} \mathrm{IP}(\mathrm{t}) \mathrm{dt} / \mathrm{T}=\mathrm{A} / 2 * \mathrm{D}{ }^{*}{ }_{{ }_{0}} \int{ }^{\mathrm{t}+\mathrm{T}} \cos (\varphi) \mathrm{dt} / \mathrm{T} \\
& \mathrm{Q}={ }_{\mathrm{PIT}} \int \mathrm{S}^{*} \mathrm{QP}(\mathrm{t}) \mathrm{dt} / \mathrm{T}=\mathrm{A} / 2 * \mathrm{D}^{*}{ }_{\mathrm{t} 0} \int{ }^{\mathrm{t}+\mathrm{T}} \sin (\varphi) \mathrm{dt} / \mathrm{T}
\end{aligned}
$$

where

$\mathrm{T}$ is the integration period or Predetection Integration Time (PIT), and

$\mathrm{D}$ is the GPS message data bit between $\mathrm{t}_{0}$ and $\mathrm{t}_{0}+\mathrm{T}$, assumed not to alternate during the integration period (see § 3.5).

Assuming that the phase deviation remains constant (i.e. changes slowly) within the integration period, the correlators outputs I and $\mathrm{Q}$ are directly proportional to the sine and cosine of the phase deviation:

$$
\begin{aligned}
& \mathrm{I}=\mathrm{A} / 2 * \mathrm{D} * \cos (\varphi) \\
& \mathrm{Q}=\mathrm{A} / 2 * \mathrm{D}^{*} \sin (\varphi)
\end{aligned}
$$

From $I$ and $Q$, the phase deviation $\varphi$ is estimated by means of a "discrimination function" (DF). Various DF can achieve the measurement of the phase deviation. An example of discrimination function is: 
$\mathrm{DF}=\operatorname{sign}(\mathrm{I}) *(\mathrm{Q})$

This DF outputs the sine of the phase deviation (i.e. directly the phase deviation itself, since $\varphi<<1$ ), proportionally scaled by the amplitude of the signal that can be estimated from I.

Using both I and Q in the DF enables phase tracking despite data bit alternations that are not known a priori. $\mathrm{Q}$ is kept to zero by the tracking loop, while I is maximum in magnitude. Both correlators outputs are inverted in case of a data bit alternation. This occurrence on $\mathrm{I}$ is detectable since $\mathrm{I}>>0$ in magnitude. Thus the data bit is determined (and the GPS message decoded), which enables the loop to be locked by driving $Q$ to zero in the right way in the NCO.

It is usual to represent graphically the correlators outputs I and Q. A phasor diagram, as in Fig. 3.5, is used. It is a 2-dimensional diagram in which the vertical axis corresponds to the value of the punctual in-phase product (I) and the horizontal axis corresponds to the value of the punctual in-quadrature product $(Q)$. I and $Q$ are here assumed to be integrated over the PIT.

The misalignment of the phasor diagram displays the phase deviation $\varphi$ to the perfectly tracked signal, for which $\varphi$ equals 0 . Note that the arrow flips to its opposite with data bit alternations.

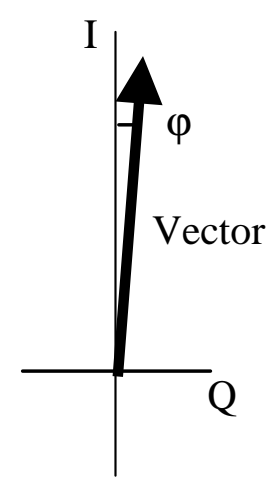

Figure 3.5: phasor diagram representation of the received signal 
It is important to bear in mind the physical reasons of the phase de-synchronisation. They are principally two: the Doppler variation and the NCO stability.

In a static application, the Doppler affected frequency is approximately represented in Fig. 3.6 [KAPLAN et al, 1996]. One can notice that the maximum Doppler variation is when the Doppler itself equals zero. The corresponding Doppler variation is around $1 \mathrm{~Hz} / \mathrm{s}$ (i.e. $0.2 \mathrm{~m} / \mathrm{s}^{2}$ with $\lambda \sim 0.2 \mathrm{~m}$ as an order of magnitude of the wavelength). In a kinematic application, severe dynamics of the GPS unit can cause vertical acceleration of 3 g (i.e. $\left.30 \mathrm{~m} / \mathrm{s}^{2}\right)$.

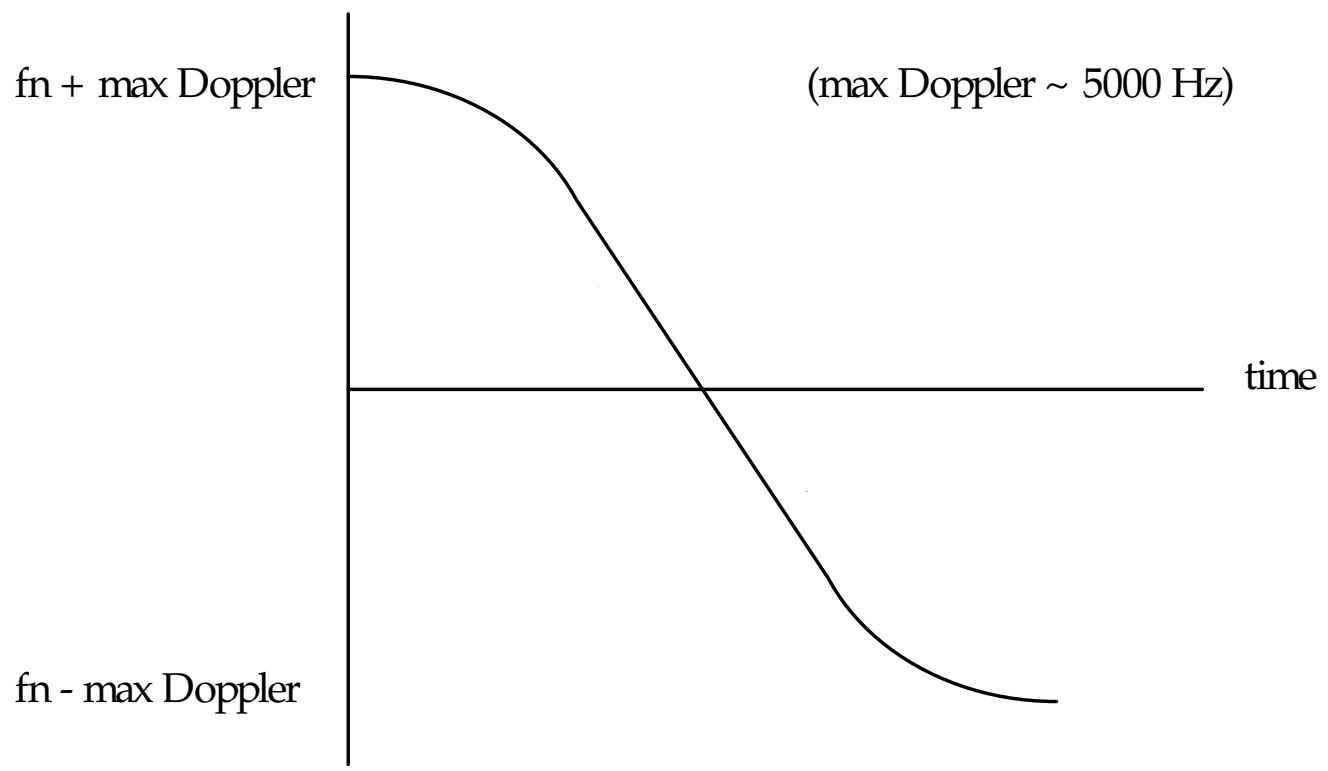

Figure 3.6: received Doppler frequency in a static application

The phase deviation due to a Doppler variation linear in time can be computed as follows. The Doppler variation is approximated by the following first-order Taylor's expansion:

$\dot{\rho}(\mathrm{t})=\dot{\rho}_{0}+\ddot{\rho}_{0} *\left(\mathrm{t}-\mathrm{t}_{0}\right)$

where

$\ddot{\rho}_{0}$ is the variation of the range-rate at $\mathrm{t}_{0}$. 
Hence, the Doppler affected intermediate frequency is given by the following function of time t:

$$
\mathrm{f}(\mathrm{t})=\mathrm{fi}-\mathrm{fn}^{*} \frac{\dot{\rho}_{0}}{\mathrm{c}}-\mathrm{fn}^{*} \frac{\ddot{\rho}_{0}}{\mathrm{c}} *\left(\mathrm{t}-\mathrm{t}_{0}\right)=\mathrm{f}_{0}-\mathrm{fn}^{*} \frac{\ddot{\rho}_{0}}{\mathrm{c}} *\left(\mathrm{t}-\mathrm{t}_{0}\right)
$$

As already given in Eq. 3.10, the phase deviation between the received signal and the replica (generated at the carrier frequency $\mathrm{f}_{0}$ ) equals:

$$
\varphi=-2 \pi^{*} \mathrm{fn} * \frac{\ddot{\rho}_{0}}{\mathrm{c}} *\left(\mathrm{t}-\mathrm{t}_{0}\right)^{2}=-2 \pi^{*} \mathrm{fn} * \frac{\ddot{\rho}_{0}}{\mathrm{c}} * \mathrm{~T}^{2}
$$

The integration period $\mathrm{T}$ in a receiver classically equals $5 \mathrm{~ms}$. So, even in kinematic applications with rover dynamics of the order of $3 \mathrm{~g}$, the term $2 \pi \mathrm{fn}^{*} \frac{\ddot{\rho}_{0}}{\mathrm{c}} * \mathrm{~T}^{2}$ equals around one degree $\left(\mathrm{fn} \sim 1.5^{\mathrm{e}} 9 \mathrm{~Hz} ; \ddot{\rho}_{0} \sim 30 \mathrm{~m} / \mathrm{s}^{2} ; \mathrm{c} \sim 3^{\mathrm{e}} 8 \mathrm{~m} / \mathrm{s}\right)$.

Hence, the approximation $\sin (\varphi) \sim \varphi$ is valid and the phase lock loop returns incremental phase adjustments that are effectively $<<1$.

As far as the NCO stability is concerned, one generally assumes that within half an hour after starting the receiver, its internal temperature is changing, which may cause the $\mathrm{NCO}$ to vary by a few thousand Hz. Afterward, both the internal temperature and the $\mathrm{NCO}$ are stable. A reasonable value for the rate of change of the $\mathrm{NCO}$ during this cold to warm transition is around $2 \mathrm{~Hz} / \mathrm{s}$. It has the same order of magnitude as the Doppler variation for static applications. On the contrary, the Doppler variation predominates for kinematic applications.

Note also that the NCO stability affects all channels, which leads to the same effects on phase measurements as the unknown receiver clock offset with GPS time.

Lastly, it is important to bear in mind that the complete process is in discrete and not continuous time. Hence, in the actual digital implementation, the low-pass filter consists in 
a summation of the correlators outputs (and averaging operation), i.e. the sum of the corresponding (8F for Leica ME) samples during the so-called "integration period".

\section{4 - Code tracking}

This section presents the main features of a standard Delay Lock Loop (DLL), which achieves code tracking and code measurements. The problem of demodulating the encrypted Y-code is not addressed, and the presentation concerns only the case of C/Acode. The functioning of a DLL is detailed step by step, for the particular "non-coherent dot-product power" type of correlator first, and also for a few other standard ones. A similar analysis can be done for any other type of correlator.

Again, suppose that signal tracking is in steady state at $t_{0} . \tau$ is the instantaneous code deviation (code error of the DLL) and $\varphi$ is the instantaneous phase deviation (phase error of the PLL) between the received signal and the replica. Each channel has fixed its own PRN code during the acquisition.

The process of code measurements is again based on ASIC hardware counting of the code adjustments that are measured and applied iteration by iteration in a controlled lock loop.

The aim of the code loop is to detect the transitions of the received code. To do so, a replica is generated in a PRN code generator and must be kept synchronised with the code carried by the received signal. Once the PRN code is synchronised, the Time Of Arrival (TOA) of the signal is determined in the receiver time reference, from which the travel time is processed and the positioning problem can be solved.

The physical reasons of the code de-synchronisation are basically the same as in the case of the phase: Doppler variation, NCO stability and - to a lesser extent - the propagation effects (that cause the "group velocity" to change).

It is interesting to notice that the transitions of the received code are predictable in time because their occurrences are due to Doppler effect on the signal, which has already 
been estimated by the phase loop. Some GPS receivers use this possibility ("carrier rate aided" mode [GREWAL et al., 2001]) particularly in order to enhance the tracking performance of the code loop in case of severe dynamics. Then, the code loop only returns incremental code adjustments due to the variation of propagation effects.

Nevertheless, both code and phase loops are clearly necessary because code and phase do not experience the same propagation effects when travelling from the satellite to the receiver antennas. In many receivers, the "carrier rate aided mode" is not implemented, and the Doppler effect is estimated twice.

The code deviation is again obtained in a correlation process. The received signal (see Eq. 3.8) is correlated with several replicas combined in an appropriate way. These replicas are:

- the punctual code in phase $(\operatorname{IP}(\mathrm{t}))$

- the $1 / 2$ chip early code in phase (IE $(\mathrm{t}))$

- the $1 / 2$ chip late code in phase (IL(t))

- the punctual code in quadrature $(\mathrm{QP}(\mathrm{t}))$

- the $1 / 2$ chip early code in quadrature $(\mathrm{QE}(\mathrm{t}))$

- the $1 / 2$ chip late code in quadrature $(\mathrm{QL}(\mathrm{t}))$

$1 / 2$ chip spacing (i.e. half the duration of the chip: $\sim 1 \mu$ s for C/A-code and $\sim 0.1 \mu \mathrm{s}$ for P-code) is the typical value of a standard "wide" correlator (see Chapter 2, § 2.2).

In a DLL, IP, IE, IL and QP, QE, QL are generated jointly by the receiver NCO (Numerically Controlled Oscillator) as far as the carrier phase is concerned, and also by the PRN code generator. The NCO is in phase with the received signal, within the phase tracking error $\varphi$ of the PLL due to the frequency difference between the carrier frequencies $\mathrm{f}$ (received) and $\mathrm{f}_{0}$ (generated at $\mathrm{t}_{0}$ ). The PRN code generator has an instantaneous code error $\tau$. 
Hence, IP, IE, IL and QP, QE, QL are generated by the receiver as follows:

$$
\begin{aligned}
& \mathrm{IP}(\mathrm{t})=\mathrm{C}(\mathrm{t}+\tau)^{*} \sin \left(2 \pi^{*} \mathrm{f}_{0}{ }^{*} \mathrm{t}\right) \\
& \operatorname{IE}(t)=C(t+\tau+1 / 2 \text { chip }) * \sin \left(2 \pi^{*} f_{0}^{*} t\right) \\
& \operatorname{IL}(\mathrm{t})=\mathrm{C}\left(\mathrm{t}+\tau-1 / 2_{\text {chip }}\right) * \sin \left(2 \pi^{*} \mathrm{f}_{0}{ }^{*} \mathrm{t}\right) \\
& \mathrm{QP}(\mathrm{t})=\mathrm{C}(\mathrm{t}+\tau)^{*} \cos \left(2 \pi^{*} \mathrm{f}_{0}^{*} \mathrm{t}\right) \\
& \mathrm{QE}(\mathrm{t})=\mathrm{C}\left(\mathrm{t}+\tau+\frac{1 / 2 \text { chip }}{}\right) * \cos \left(2 \pi^{*} \mathrm{f}_{0}{ }^{*} \mathrm{t}\right) \\
& \mathrm{QL}(\mathrm{t})=\mathrm{C}\left(\mathrm{t}+\tau-1 / 2_{\text {chip }}\right) * \cos \left(2 \pi^{*} \mathrm{f}_{0}{ }^{*} \mathrm{t}\right)
\end{aligned}
$$

Thus:

$$
\begin{array}{r}
S^{*} \operatorname{IP}(t)=A D(t)^{*} \sin \left(2 \pi^{*} f^{*} t\right)^{*} \sin \left(2 \pi^{*} f_{0}^{*} t\right)^{*} C(t)^{*} C(t+\tau) \\
=A D(t)^{*}\left(\cos \left(2 \pi^{*}\left(f-f_{0}\right)^{*} t\right)-\cos \left(2 \pi^{*}\left(f+f_{0}\right)^{*} t\right)\right) / 2 * R(\tau)
\end{array}
$$

$$
\begin{array}{r}
S^{*} \operatorname{IE}(t)=A D(t) * \sin \left(2 \pi^{*} f^{*} t\right)^{*} \sin \left(2 \pi^{*} f_{0}^{*} t\right)^{*} C(t)^{*} C(t+\tau+1 / 2 \text { chip }) \\
\quad=A D(t)^{*}\left(\cos \left(2 \pi^{*}\left(f-f_{0}\right)^{*} t\right)-\cos \left(2 \pi^{*}\left(f+f_{0}\right)^{*} t\right)\right) / 2 * R\left(\tau+\frac{1}{2} \text { chip }\right)
\end{array}
$$

$$
\begin{aligned}
& S^{*} \operatorname{IL}(t)=A D(t) * \sin \left(2 \pi^{*} f^{*} t\right)^{*} \sin \left(2 \pi^{*} f_{0}^{*} t\right)^{*} C(t)^{*} C(t+\tau-1 / 2 \text { chip }) \\
& \left.\quad=\operatorname{AD}(t) *\left(\cos \left(2 \pi^{*}\left(f-f_{0}\right)\right)^{*} t\right)-\cos \left(2 \pi^{*}\left(f+f_{0}\right)^{*} t\right)\right) / 2 * R(\tau-1 / 2 \text { chip })
\end{aligned}
$$

$$
\begin{gathered}
S^{*} \mathrm{QP}(\mathrm{t})=\mathrm{AD}(\mathrm{t})^{*} \sin \left(2 \pi^{*} \mathrm{f}^{*} \mathrm{t}\right)^{*} \cos \left(2 \pi^{*} \mathrm{f}_{0}^{*} \mathrm{t}\right){ }^{*} \mathrm{C}(\mathrm{t})^{*} \mathrm{C}(\mathrm{t}+\tau) \\
\quad=\mathrm{AD}(\mathrm{t})^{*}\left(\sin \left(2 \pi^{*}\left(\mathrm{f}+\mathrm{f}_{0}\right)^{*} \mathrm{t}\right)-\sin \left(2 \pi^{*}\left(\mathrm{f}-\mathrm{f}_{0}\right)^{*} \mathrm{t}\right)\right) / 2 * \mathrm{R}(\tau)
\end{gathered}
$$

$$
\begin{gathered}
S^{*} Q E(t)=A D(t)^{*} \sin \left(2 \pi^{*} f^{*} t\right)^{*} \cos \left(2 \pi^{*} f_{0}^{*} t\right) * C(t)^{*} C(t+\tau+1 / 2 \text { chip }) \\
\quad=A D(t)^{*}\left(\sin \left(2 \pi^{*}\left(f+f_{0}\right)^{*} t\right)-\sin \left(2 \pi^{*}\left(f-f_{0}\right)^{*} t\right)\right) / 2 * R(\tau+1 / 2 \text { chip })
\end{gathered}
$$

$S^{*} Q L(t)=A D(t)^{*} \sin \left(2 \pi^{*} f^{*} t\right)^{*} \cos \left(2 \pi^{*} f_{0}^{*} t\right) * C(t)^{*} C(t+\tau-1 / 2$ chip $)$

$$
=A D(t) *\left(\sin \left(2 \pi^{*}\left(f+f_{0}\right)^{*} t\right)-\sin \left(2 \pi^{*}\left(f-f_{0}\right)^{*} t\right)\right) / 2 * R(\tau-1 / 2 \text { chip })
$$


The instantaneous phase error $\varphi$ results from the frequency difference between $\mathrm{f}$ and $\mathrm{f}_{0}$ and it is controlled by the PLL. The high-frequency term $\left(\mathrm{f}+\mathrm{f}_{0}\right)$ is filtered.

$$
\begin{aligned}
& I={ }_{\text {PIT }} \int S^{*} \mathrm{IP}(\mathrm{t}) \mathrm{dt} / \mathrm{T}=\mathrm{A} / 2{ }^{*} \mathrm{D}^{*} \cos (\varphi) * \mathrm{R}(\tau) \\
& \mathrm{IE}={ }_{\text {PIT }} \int \mathrm{S}^{*} \mathrm{IE}(\mathrm{t}) \mathrm{dt} / \mathrm{T}=\mathrm{A} / 2{ }^{*} \mathrm{D}^{*} \cos (\varphi) * \mathrm{R}\left(\tau+1 / 2_{\text {chip }}\right) \\
& \mathrm{IL}={ }_{\text {PIT }} \int \mathrm{S}^{*} \mathrm{IL}(\mathrm{t}) \mathrm{dt} / \mathrm{T}=\mathrm{A} / 2 * \mathrm{D}^{*} \cos (\varphi) * \mathrm{R}\left(\tau-1 / 2_{\text {chip }}\right) \\
& \mathrm{Q}={ }_{\text {PIT }} \int \mathrm{S}^{*} \mathrm{QP}(\mathrm{t}) \mathrm{dt} / \mathrm{T}=\mathrm{A} / 2{ }^{*} \mathrm{D}^{*} \sin (\varphi) * \mathrm{R}(\tau) \\
& \mathrm{QE}={ }_{\text {PIT }} \int \mathrm{S}^{*} \mathrm{QE}(\mathrm{t}) \mathrm{dt} / \mathrm{T}=\mathrm{A} / 2{ }^{*} \mathrm{D}^{*} \sin (\varphi) * \mathrm{R}\left(\tau+1 / 2_{\text {chip }}\right) \\
& \mathrm{QL}={ }_{\text {PIT }} \int \mathrm{S}^{*} \mathrm{QL}(\mathrm{t}) \mathrm{dt} / \mathrm{T}=\mathrm{A} / 2 * \mathrm{D}^{*} \sin (\varphi) * \mathrm{R}(\tau-1 / 2 \text { chip })
\end{aligned}
$$

In order to assess to the code tracking error, the following discrimination function (called "non-coherent dot-product power") is formed, after the correlator outputs have been filtered. This is developed in Eq. 3.21:

$$
\begin{aligned}
\mathrm{DF} & =\mathrm{I}(\mathrm{IE}-\mathrm{IL})+\mathrm{Q}(\mathrm{QE}-\mathrm{QL}) \\
& =\mathrm{A}^{2} / 4 * \mathrm{D}^{2} * \mathrm{R}(\tau) *\left[\mathrm{R}\left(\tau+\frac{1}{2_{\text {chip }}}\right)-\mathrm{R}\left(\tau-1 / 2_{\text {chip }}\right)\right] *\left(\cos ^{2} \varphi+\sin ^{2} \varphi\right) \\
& =\mathrm{A}^{2} / 4 * \mathrm{R}(\tau) *\left[\mathrm{R}\left(\tau+1 / 2_{\text {chip }}\right)-\mathrm{R}\left(\tau-1 / 2_{\text {chip }}\right)\right]
\end{aligned}
$$

Note that $\varphi$ disappears in Eq. 3.21, which means that this DF is independent of any possible phase tracking error. For this reason, this correlator is described as "noncoherent". Note also that $\mathrm{D}^{2}=1$. Consequently, the data bit alternation does not affect a code loop using this DF.

Finally, $\mathrm{DF}=\mathrm{A}^{2} / 4 * \mathrm{R}(\tau) *[\mathrm{R}(\tau+1 / 2)-\mathrm{R}(\tau-1 / 2)]$ enables the code loop to be controlled. The discrimination function is represented in Fig. 3.7, as a function of code deviation to the perfectly tracked signal. Note that the zero point of $\mathrm{DF}(\mathrm{DF}=0)$ actually corresponds to perfect tracking $(\tau=0)$. 
Another common discrimination function is:

$$
\begin{aligned}
\mathrm{DF} & =\sqrt{ }\left((\mathrm{IE})^{2}+(\mathrm{QE})^{2}\right)-\sqrt{ }\left((\mathrm{IL})^{2}+(\mathrm{QL})^{2}\right) \\
& =\mathrm{A} / 2 *[\mathrm{R}(\tau+1 / 2)-\mathrm{R}(\tau-1 / 2)]
\end{aligned}
$$

This DF is called "non-coherent early-late envelope". Except for the term dependent on the amplitude of the signal (that can be normalised), this second DF differs from the "non-coherent dot-product power" DF by the product term $\mathrm{R}(\tau)$. Hence, there is a difference in the discriminator output. Both are represented in the next figure, once normalised by respectively $\mathrm{A}^{2} / 4$ power and $\mathrm{A} / 2$ amplitude.

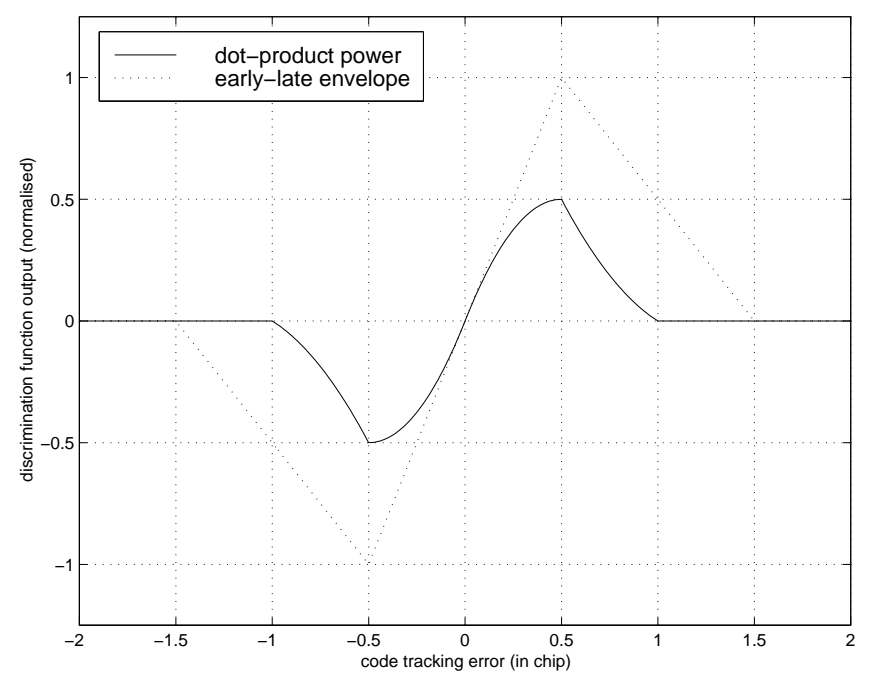

Figure 3.7: different code discrimination functions

Note that when the generated signal is perfectly in phase with the received signal (i.e. when the phase loop is locked), the outputs of the correlators in quadrature (QP, QE and QL) equal 0. Then the preceding discrimination functions can be simplified to their equivalents in a coherent form. In the case of the "coherent early-late envelope", the discrimination function has the most elementary form:

$$
\mathrm{DF}=\mathrm{IE}-\mathrm{IL}
$$


which has the advantage of the lowest through-put.

In most receivers, the non-coherent form of the discrimination function is used only before the phase loop is locked, afterward the coherent form is switched on.

In this later case, the data bit alternations must be input from the PLL (where they are determined) into the DLL. Thus, the sign of the discrimination function is determined, which enables the loop to be locked by driving IE-IL to zero in the right way in the PRN code generator.

Lastly, in the actual $40 \mathrm{MHz}$ digital implementation, which gives 40 samples per chip, the difference between the received and generated codes can only be corrected to the closest physical clock edge. So in practice the code adjustments in the controlled lock loop are made by the minimum step of $1 / 40^{\text {th }}$ chip only. As soon as the code adjustments get over half of this elementary chip interval, a sample is suppressed or inserted in the generated code. Hence, the code transitions are never synchronised to the actual samples, but slide between the receiver clock edges. Note that, whenever the code adjustments are made, the code measurements are available at the code loop integration period.

\section{5 - Integration period}

The integration period is the duration of the summation and averaging operation carried out in the code and phase lock-loops in order to suppress the high-frequency term by low-pass filtering. It is also called the Predetection Integration Time (PIT).

It also corresponds to the feed-back period at which the incremental adjustments are returned to enable the control of the lock-loops.

In the phase loop, the integration period is limited by the duration of the data bit: the message frequency is $50 \mathrm{~Hz}$, i.e. $20 \mathrm{~ms}$. The integration period maximum value is the data bit duration, $20 \mathrm{~ms}$.

In the code loop: 
- if it is coherent, the discrimination function eliminates the data bit, so there is no limit in the integration period due to the data bit;

- if it is non-coherent, the limit of $20 \mathrm{~ms}$ can be overcome, because the phase loop is locked in this case and the data bit is determined at any time. If the data bit is copied from the phase loop to the code loop, then the integration period can exceed the data bit duration.

The choice of a value for the PIT is a problem to be solved in receiver design. It must be fixed with respect to the balance between:

- on the one hand, the interest in increasing the integration period, to make the measurements less sensitive to noise, and on the other hand,

- the interest in decreasing the integration period, to permit tracking when the rover receiver is subjected to high dynamics. The expected capability of a loop with respect to dynamics has direct consequence on its design, i.e. on its order and bandwidth, and on the integration period as a consequence.

A predetection integration time of $5 \mathrm{~ms}$ is adequate to feed the phase loop as this usually has a bandwidth of around $20 \mathrm{~Hz}(26 \mathrm{~Hz}$ in the Leica ME). This is because raw measurements in the correlator are in practice made at a frequency at least 10 times the bandwidth of the corresponding loop. Note that a $20 \mathrm{~Hz}$ bandwidth phase loop permits an output of $20 \mathrm{~Hz}$ independent phase data.

Moreover, within the $5 \mathrm{~ms}$ duration of the integration period, the phase difference between the received signal and its replica does not vary by more than about 1 degree. This has been demonstrated by computing the variation of the Doppler in time for a rover with an acceleration of $3 \mathrm{~g}$, which is the maximum acceptable acceleration of the rover with the $26 \mathrm{~Hz}$ bandwidth $2^{\text {nd }}$ order phase loop of the Leica ME.

As far as the code loop is concerned, Leica ME characteristics are $0.31 \mathrm{~Hz}$ bandwidth $1^{\text {st }}$ order. The code loop could be fed at only $5 \mathrm{~Hz}$, but the design choice was $50 \mathrm{~Hz}$, to keep to the limit due to the $20 \mathrm{~ms}$ data bit duration. 
Chapter 4

PHASE AND CODE TRACKING IN THE PRESENCE OF MULTIPATH

Both GPS code and carrier phase measurements are contaminated by multipath, but the characteristics of multipath errors in measurements are different. Thereafter, these errors are presented in detail, considering firstly phase multipath and secondly code multipath.

\section{1 - Phase tracking in the presence of multipath}

As already introduced in Chapter $1, \S 1.2 .4$, it is common to characterise the received signal by the addition (or combination) of the direct and reflected signals:

$S_{\text {direct }}=S d=A D(t) * C(t) * \sin \left(2 \pi^{*} f^{*} t\right)$

$\mathrm{S}_{\text {reflected }}=\mathrm{Sr}=\Sigma_{\mathrm{i}} \alpha_{\mathrm{i}} \mathrm{A} * \mathrm{D}\left(\mathrm{t}-\mathrm{d}_{\mathrm{i}}\right) * \mathrm{C}\left(\mathrm{t}-\mathrm{d}_{\mathrm{i}}\right) * \sin \left(2 \pi^{*} \mathrm{f}^{*} \mathrm{t}-\Theta_{\mathrm{i}}\right)$

where
A is the amplitude of the direct signal
$\mathrm{D}$ is the received GPS message data bit time series
$C$ is the received PRN code time series
$\mathrm{f}$ is the carrier frequency
$\alpha_{i}$ is the ratio of amplitude of the direct and the $i^{\text {th }}$ reflected signals
$\mathrm{d}_{\mathrm{i}}$ is the delay of the $\mathrm{i}^{\text {th }}$ reflected signal (i.e. the additional path length $\mathrm{L}_{\mathrm{i}} / \mathrm{c}$ ), and
$\Theta_{i}$ is the phase shift of $i^{\text {th }}$ reflected signal. 
A varies in time with the elevation of the satellite relatively to the plane of the antenna, in accordance with the antenna gain pattern in right-hand polarisation.

The phase of the direct signal $\left(\Phi=2 \pi^{*} f^{*} t\right)$ is directly linked to the frequency (f1 or $\mathrm{f} 2$ ) of the carrier wave, and its Doppler variation in time.

The ratio of amplitude $\alpha$ depends on the coefficient of reflection of the reflective surface (so the material of which it is made), as quoted in Chapter 1, §1.2.2, and also fundamentally on the antenna gain pattern $(\S 1.2 .3)$ in both right-hand and left-hand polarisations. Therefore, $\alpha$ in general will change with the reflecting surface and the type of antenna. For a given reflecting surface and antenna, it will also vary with the elevation (and the azimuth for a non micro-centred antenna) of both the direct and the reflected signals on the plane of the antenna. Hence, it is necessary to bear in mind that $\alpha$ is not a constant. When this ratio is considered as a constant, its value is linked to a certain angular domain within the antenna gain patterns in right-hand and left-hand polarisations. Then this value cannot be generalised to all possible relative geometries of the antenna and the reflecting surface.

The delay $\mathrm{d}$ is positive in this model. It can be deduced from the additional path length $\mathrm{L}$ by a geometrical computation $(\mathrm{d}=\mathrm{L} / \mathrm{c})$. The phase shift $\Theta$ is obviously related to $\mathrm{L}$ and the wavelength $(\Theta=2 \pi \mathrm{L} / \lambda)$. But it also depends on the physical electromagnetic properties of the reflecting surface that affect the carrier phase itself independently of the delay. So one must consider also the reflection phase shift $\left(180^{\circ}\right.$ or 0 depending on whether the grazing angle is respectively above or below the Brewster angle, see Chapter 1 , $\S 1.2 .2)$. Lastly, it also depends on the antenna phase pattern (§ 1.2.3).

These signals are combined in case of multipath, which means that they add together in the signal processing carried out in the receiver.

$\mathrm{S}_{\text {combined }}=\mathrm{Sc}=\mathrm{Sd}+\mathrm{Sr}$ 
Chapter 3 has shown that the PLL is continuously tracking the received signal, which is now composed of mixed direct and reflected signals. The discrimination function DF of the phase loop is computed with mixed signals, and Eq. 4.7 shows that multipath entails a certain bias in the phase error $\varphi$ of the PLL derived from the equation DF $=0$.

The received signal is:

$$
S=S c=A\left\{D(t) * C(t) * \sin \left(2 \pi^{*} f^{*} t\right)+\Sigma_{i} \alpha_{i}^{*} D\left(t-d_{i}\right) * C\left(t-d_{i}\right) * \sin \left(2 \pi^{*} f^{*} t-\Theta_{i}\right)\right\}
$$

Suppose signal tracking in steady state. The received signal is correlated with:

-the punctual code in phase $\left(\operatorname{IP}(t)=C(t+\tau)^{*} \sin \left(2 \pi^{*} f_{0}^{*} t\right)\right)$, and

- the punctual code in quadrature $\left(\mathrm{QP}(\mathrm{t})=\mathrm{C}(\mathrm{t}+\tau) * \cos \left(2 \pi^{*} \mathrm{f}_{0}^{*} \mathrm{t}\right)\right)$

as already explained in Eq. 3.11. Hence,

$$
\begin{aligned}
& \mathrm{S}^{*} \mathrm{IP}(\mathrm{t})=\mathrm{A} / 2\left\{\mathrm{D}(\mathrm{t})^{*}\left(\cos \left(2 \pi^{*}\left(\mathrm{f}-\mathrm{f}_{0}\right)^{*} \mathrm{t}\right)-\cos \left(2 \pi^{*}\left(\mathrm{f}+\mathrm{f}_{0}\right)^{*} \mathrm{t}\right)\right)^{*} \mathrm{R}(\tau)\right. \\
& \left.\quad+\Sigma_{\mathrm{i}} \alpha_{\mathrm{i}}^{*} \mathrm{D}\left(\mathrm{t}-\mathrm{d}_{\mathrm{i}}\right) *\left(\cos \left(2 \pi^{*}\left(\mathrm{f}-\mathrm{f}_{0}\right)^{*} \mathrm{t}-\Theta_{\mathrm{i}}\right)-\cos \left(2 \pi^{*}\left(\mathrm{f}+\mathrm{f}_{0}\right)^{*} \mathrm{t}-\Theta_{\mathrm{i}}\right)\right) * \mathrm{R}\left(\tau+\mathrm{d}_{\mathrm{i}}\right)\right\} \\
& \mathrm{S}^{*} \mathrm{QP}(\mathrm{t})=\mathrm{A} / 2\left\{\mathrm{D}(\mathrm{t})^{*}\left(\sin \left(2 \pi^{*}\left(\mathrm{f}+\mathrm{f}_{0}\right)^{*} \mathrm{t}\right)-\sin \left(2 \pi^{*}\left(\mathrm{f}-\mathrm{f}_{0}\right)^{*} \mathrm{t}\right)\right)^{*} \mathrm{R}(\tau)\right. \\
& \left.\quad+\Sigma_{\mathrm{i}} \alpha_{\mathrm{i}}^{*} \mathrm{D}\left(\mathrm{t}-\mathrm{d}_{\mathrm{i}}\right)^{*}\left(\sin \left(2 \pi^{*}\left(\mathrm{f}+\mathrm{f}_{0}\right)^{*} \mathrm{t}-\Theta_{\mathrm{i}}\right)-\sin \left(2 \pi^{*}\left(\mathrm{f}-\mathrm{f}_{0}\right)^{*} \mathrm{t}-\Theta_{\mathrm{i}}\right)\right)^{*} \mathrm{R}\left(\tau+\mathrm{d}_{\mathrm{i}}\right)\right\}
\end{aligned}
$$

In the ASIC (see Eq. 3.13), the sum frequency term $\left(f+f_{0}\right)$ can be low-pass filtered. The difference frequency term $\left(\mathrm{f}-\mathrm{f}_{0}\right)$ corresponds to the Doppler variation in time, and leads to the phase error $\varphi$ of the phase tracking loop. To do so, the preceding equations are averaged over the integration period $\mathrm{T}$, which gives:

$$
\begin{aligned}
& \mathrm{I}={ }_{\text {PIT }} \int \mathrm{S}^{*} \mathrm{IP}(\mathrm{t}) \mathrm{dt} / \mathrm{T}=\mathrm{A} / 2 * \mathrm{D} *\left\{\cos (\varphi) * \mathrm{R}(\tau)+\Sigma_{\mathrm{i}} \alpha_{\mathrm{i}}^{*} \cos \left(\varphi-\Theta_{\mathrm{i}}\right) * \mathrm{R}\left(\tau+\mathrm{d}_{\mathrm{i}}\right)\right\} \\
& \mathrm{Q}={ }_{\mathrm{PIT}} \int \mathrm{S}^{*} \mathrm{QP}(\mathrm{t}) \mathrm{dt} / \mathrm{T}=\mathrm{A} / 2 * \mathrm{D} *\left\{\sin (\varphi) * \mathrm{R}(\tau)+\Sigma_{\mathrm{i}} \alpha_{\mathrm{i}}{ }^{*} \sin \left(\varphi-\Theta_{\mathrm{i}}\right) * \mathrm{R}\left(\tau+\mathrm{d}_{\mathrm{i}}\right)\right\}
\end{aligned}
$$

The discrimination function $\mathrm{DF}=\operatorname{sign}(\mathrm{I}) *(\mathrm{Q})$ is computed. The PLL is controlled so that the DF is kept to zero. Rearranging DF $=0$ gives successively: 


$$
\begin{aligned}
& \sin (\varphi) * R(\tau)+\sum_{i} \alpha_{i}^{*} \sin \left(\varphi-\Theta_{i}\right) * R\left(\tau+d_{i}\right)=0 \\
& \sin (\varphi) * R(\tau)+\sum_{i} \alpha_{i}^{*}\left(\sin (\varphi) \cos \left(\Theta_{i}\right)-\cos (\varphi) \sin \left(\Theta_{i}\right)\right) * R\left(\tau+d_{i}\right)=0 \\
& \sin (\varphi) *\left[R(\tau)+\Sigma_{i} \alpha_{i}^{*} \cos \left(\Theta_{i}\right) * R\left(\tau+d_{i}\right)\right]-\cos (\varphi) *\left[\Sigma_{i} \alpha_{i}^{*} \sin \left(\Theta_{i}\right) * R\left(\tau+d_{i}\right)\right]=0 \\
& \text { and finally. }
\end{aligned}
$$

$$
\tan (\varphi)=\left[\Sigma_{\mathrm{i}} \alpha_{\mathrm{i}}^{*} \sin \left(\Theta_{\mathrm{i}}\right) * \mathrm{R}\left(\tau+\mathrm{d}_{\mathrm{i}}\right)\right] /\left[\mathrm{R}(\tau)+\Sigma_{\mathrm{i}} \alpha_{\mathrm{i}}{ }^{*} \cos \left(\Theta_{\mathrm{i}}\right) * \mathrm{R}\left(\tau+\mathrm{d}_{\mathrm{i}}\right)\right]
$$

Let us define the so-called modified ratio of amplitude:

$$
\alpha_{i}^{\prime}=\alpha_{i}^{*} R\left(\tau+d_{i}\right) / R(\tau)
$$

Hence, the phase loop error (or phase adjustment) is:

$$
\varphi=\arctan \left(\left[\Sigma_{\mathrm{i}} \alpha_{\mathrm{i}}^{\prime *} \sin \left(\Theta_{\mathrm{i}}\right)\right] /\left[1+\Sigma_{\mathrm{i}} \alpha_{\mathrm{i}}^{\prime *} \cos \left(\Theta_{\mathrm{i}}\right)\right]\right)=\varphi_{\mathrm{m}} \neq 0
$$

So it appears that, in the presence of multipath (and contrary to the ideal situation where the phase loop error would result from the direct signal only), the equation $\mathrm{DF}=0$ does not lead anymore to $\varphi=0$, but to $\varphi=\varphi_{\mathrm{m}} \neq 0$. The discrimination function has been shifted by multipath and its zero point does not correspond to perfect tracking (where the phase loop error $\varphi$ should be zero). The phase loop error becomes biased $\left(\varphi=\varphi_{m} \neq 0\right)$, as well as the phase measurements that result from hardware counting of the phase adjustments.

Moreover, the dependency between phase and code loops (and consequently the dependency between phase and code multipath errors) is shown in Eq. 4.9 through the modified ratio of amplitude defined in Eq. 4.8, where $\tau$ appears and is also biased $\left(\tau=\tau_{\mathrm{m}} \neq 0\right)$. This will be demonstrated in $\S 4$.2.

A phasor representation (where the correlator outputs I and $Q$ are displayed along the vertical and horizontal axes) is worthwhile to complete the mathematical derivation of the multipath phase error. This representation is here given in the case of a single reflected signal. The alignment of the phasor diagram generally entails a phase error $\varphi_{m}$ (except if the 
phase shift of the reflected signal $\Theta$ equals 0 or $180^{\circ}$ ). Let us recall that $\Theta$ (and $\varphi_{m}$ as a matter of fact) varies with the satellite position through the additional path length $\mathrm{L}$ travelled by the reflected wave. So the shape of the phasor is continuously changing.

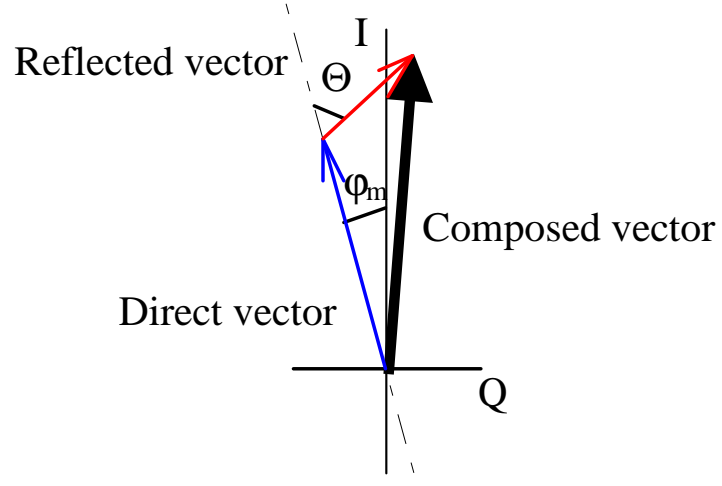

Figure 4.1: phasor diagram representation of the composed (direct + reflected) signal

Maximum phase errors occur when the vector representing the reflected signal is perpendicular to the one representing the received signal (see Fig. 4.1).

Neglecting the fact that $\tau$ is affected by multipath $\left(\tau=\tau_{\mathrm{m}} \neq 0\right)$ and depends on $\Theta$, the derivation of Eq. 4.9 with $\Theta$ gives:

$\Theta= \pm \arccos \left(-\alpha^{\prime}\right)$

as the two occurrences of the maximum phase errors, which are:

$\varphi_{\mathrm{m}}= \pm \arctan \left(\alpha^{\prime} / \sqrt{ }\left(\left(1-\left(\alpha^{\prime}\right)^{2}\right)\right)= \pm \arcsin \left(\alpha^{\prime}\right)\right.$

where

$\alpha^{\prime}$ is the modified ratio of amplitude. 
It should be noted that for reflection with small code error $\left(\tau=\tau_{\mathrm{m}} \sim 0\right)$ like when using a narrow code correlator (see $§ 4.2 .2$ ):

$\alpha^{\prime}=\alpha^{*} \mathrm{R}(\tau+\mathrm{d}) / \mathrm{R}(\tau)=\alpha^{*}(1-|\tau+\mathrm{d}| / \mathrm{T}) /(1-|\tau| / \mathrm{T}) \sim \alpha^{*}(1-\mathrm{d} / \mathrm{T})$

where $\mathrm{d}$ and $\tau$ are normalised by the duration T of the chip ( $\mathrm{d}$ is positive). Moreover, the assumption ( $\tau$ independent of $\Theta$ ) is valid with a small code error.

So, the maximum phase error $\varphi_{\mathrm{m}}$ is proportional to $(1-\mathrm{d} / \mathrm{T})$, where $\mathrm{d}$ is the code delay (normalised by the duration $\mathrm{T}$ of the chip) of the reflected signal. The less the reflected signal is delayed, the more is the phase error. Fig. 4.2 displays the phase error envelope, in the case $\alpha=0.5$.

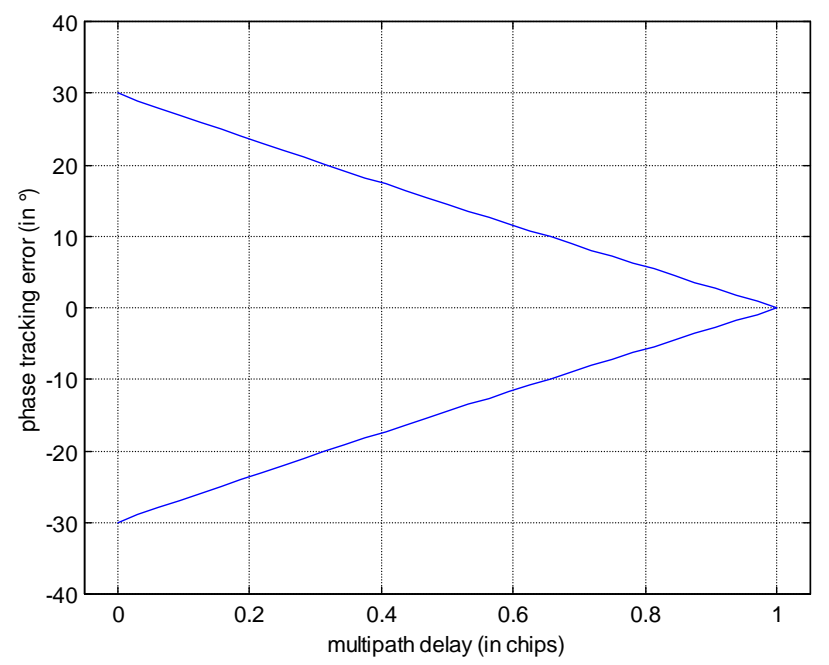

Figure 4.2: maximum phase tracking errors in the presence of multipath

\section{2 - Code tracking in the presence of multipath}

\subsection{1 - Case of the "wide" correlator code tracking}

The DLL is continuously tracking the received signal, whose representation is given in Eq. 4.4. The discrimination function DF of the code loop is computed with mixed signals, 
and Eq. 4.19 shows that multipath entails a certain bias in the code error $\tau$ of the DLL derived from the equation $\mathrm{DF}=0$. Suppose signal tracking in steady state. The received signal (composed of direct and reflected signals) is correlated with:

- the punctual code in phase (IP(t))

- the $1 / 2$ chip early code in phase (IE(t))

- the $1 / 2$ chip late code in phase (IL(t))

- the punctual code in quadrature $(\mathrm{QP}(\mathrm{t}))$

- the $1 / 2$ chip early code in quadrature $(\mathrm{QE}(\mathrm{t}))$

- the $1 / 2$ chip late code in quadrature $(\mathrm{QL}(\mathrm{t}))$

as already explained in Eq. 3.18. The term "wide" correlator stands for the $1 / 2$ chip spacing used in the generated replicas. Hence,

$$
\begin{aligned}
& S^{*} I P(t)=A / 2\left\{D(t) *\left(\cos \left(2 \pi^{*}\left(f-f_{0}\right)^{*} t\right)-\cos \left(2 \pi^{*}\left(f+f_{0}\right)^{*} t\right)\right)^{*} R(\tau)\right. \\
& \left.+\Sigma_{\mathrm{i}} \alpha_{\mathrm{i}}^{*} \mathrm{D}\left(\mathrm{t}-\mathrm{d}_{\mathrm{i}}\right) *\left(\cos \left(2 \pi^{*}\left(\mathrm{f}-\mathrm{f}_{0}\right)^{*} \mathrm{t}-\Theta_{\mathrm{i}}\right)-\cos \left(2 \pi^{*}\left(\mathrm{f}+\mathrm{f}_{0}\right)^{*} \mathrm{t}-\Theta_{\mathrm{i}}\right)\right) * \mathrm{R}\left(\tau+\mathrm{d}_{\mathrm{i}}\right)\right\} \\
& S^{*} \operatorname{IE}(t)=A / 2\left\{D(t) *\left(\cos \left(2 \pi^{*}\left(f-f_{0}\right)^{*} t\right)-\cos \left(2 \pi^{*}\left(f+f_{0}\right)^{*} t\right)\right) * R(\tau+1 / 2 \text { chip })\right. \\
& \left.+\Sigma_{\mathrm{i}} \alpha_{\mathrm{i}}^{*} \mathrm{D}\left(\mathrm{t}-\mathrm{d}_{\mathrm{i}}\right)^{*}\left(\cos \left(2 \pi^{*}\left(\mathrm{f}-\mathrm{f}_{0}\right)^{*} \mathrm{t}-\Theta_{\mathrm{i}}\right)-\cos \left(2 \pi^{*}\left(\mathrm{f}+\mathrm{f}_{0}\right)^{*} \mathrm{t}-\Theta_{\mathrm{i}}\right)\right) * \mathrm{R}\left(\tau+\mathrm{d}_{\mathrm{i}}+1 / 2 \text { chip }\right)\right\} \\
& S^{*} \operatorname{IL}(\mathrm{t})=\mathrm{A} / 2\left\{\mathrm{D}(\mathrm{t}) *\left(\cos \left(2 \pi^{*}\left(\mathrm{f}-\mathrm{f}_{0}\right)^{*} \mathrm{t}\right)-\cos \left(2 \pi^{*}\left(\mathrm{f}+\mathrm{f}_{0}\right)^{*} \mathrm{t}\right)\right) * \mathrm{R}\left(\tau-1 / 2_{\text {chip }}\right)\right. \\
& \left.+\Sigma_{\mathrm{i}} \alpha_{\mathrm{i}}^{*} \mathrm{D}\left(\mathrm{t}-\mathrm{d}_{\mathrm{i}}\right)^{*}\left(\cos \left(2 \pi^{*}\left(\mathrm{f}-\mathrm{f}_{0}\right)^{*} \mathrm{t}-\Theta_{\mathrm{i}}\right)-\cos \left(2 \pi^{*}\left(\mathrm{f}+\mathrm{f}_{0}\right)^{*} \mathrm{t}-\Theta_{\mathrm{i}}\right)\right)^{*} \mathrm{R}\left(\tau+\mathrm{d}_{\mathrm{i}}-1 / 2 \text { chip }\right)\right\} \\
& \mathrm{S}^{*} \mathrm{QP}(\mathrm{t})=\mathrm{A} / 2\left\{\mathrm{D}(\mathrm{t}) *\left(\sin \left(2 \pi^{*}\left(\mathrm{f}+\mathrm{f}_{0}\right)^{*} \mathrm{t}\right)-\sin \left(2 \pi^{*}\left(\mathrm{f}-\mathrm{f}_{0}\right)^{*} \mathrm{t}\right)\right)^{*} \mathrm{R}(\tau)\right. \\
& \left.+\Sigma_{\mathrm{i}} \alpha_{\mathrm{i}}^{*} \mathrm{D}\left(\mathrm{t}-\mathrm{d}_{\mathrm{i}}\right) *\left(\sin \left(2 \pi^{*}\left(\mathrm{f}+\mathrm{f}_{0}\right)^{*} \mathrm{t}-\Theta_{\mathrm{i}}\right)-\sin \left(2 \pi^{*}\left(\mathrm{f}-\mathrm{f}_{0}\right)^{*} \mathrm{t}-\Theta_{\mathrm{i}}\right)\right) * \mathrm{R}\left(\tau+\mathrm{d}_{\mathrm{i}}\right)\right\} \\
& S^{*} \mathrm{QE}(\mathrm{t})=\mathrm{A} / 2\left\{\mathrm{D}(\mathrm{t}) *\left(\sin \left(2 \pi^{*}\left(\mathrm{f}+\mathrm{f}_{0}\right)^{*} \mathrm{t}\right)-\sin \left(2 \pi^{*}\left(\mathrm{f}-\mathrm{f}_{0}\right)^{*} \mathrm{t}\right)\right) * \mathrm{R}\left(\tau+1 / 2_{\text {chip }}\right)\right. \\
& \left.+\Sigma_{\mathrm{i}} \alpha_{\mathrm{i}}^{*} \mathrm{D}\left(\mathrm{t}-\mathrm{d}_{\mathrm{i}}\right) *\left(\sin \left(2 \pi^{*}\left(\mathrm{f}+\mathrm{f}_{0}\right)^{*} \mathrm{t}-\Theta_{\mathrm{i}}\right)-\sin \left(2 \pi^{*}\left(\mathrm{f}-\mathrm{f}_{0}\right)^{*} \mathrm{t}-\Theta_{\mathrm{i}}\right)\right)^{*} \mathrm{R}\left(\tau+\mathrm{d}_{\mathrm{i}}+1 / 2 \text { chip }\right)\right\} \\
& S^{*} \mathrm{QL}(\mathrm{t})=\mathrm{A} / 2\left\{\mathrm{D}(\mathrm{t}) *\left(\sin \left(2 \pi^{*}\left(\mathrm{f}+\mathrm{f}_{0}\right) * t\right)-\sin \left(2 \pi^{*}\left(\mathrm{f}-\mathrm{f}_{0}\right)^{*} \mathrm{t}\right)\right) * \mathrm{R}\left(\tau-1 / 2_{\text {chip }}\right)\right. \\
& \left.+\Sigma_{\mathrm{i}} \alpha_{\mathrm{i}}{ }^{*} \mathrm{D}\left(\mathrm{t}-\mathrm{d}_{\mathrm{i}}\right) *\left(\sin \left(2 \pi^{*}\left(\mathrm{f}+\mathrm{f}_{0}\right) * \mathrm{t}-\Theta_{\mathrm{i}}\right)-\sin \left(2 \pi^{*}\left(\mathrm{f}-\mathrm{f}_{0}\right)^{*} \mathrm{t}-\Theta_{\mathrm{i}}\right)\right) * \mathrm{R}\left(\tau+\mathrm{d}_{\mathrm{i}}-1 / 2 \text { chip }\right)\right\}
\end{aligned}
$$


The preceding equations are averaged over the integration period $\mathrm{T}$, which gives:

$$
\begin{aligned}
& \mathrm{IP}=\mathrm{A} / 2 * \mathrm{D}^{*}\left\{\cos (\varphi) * \mathrm{R}(\tau)+\Sigma_{\mathrm{i}} \alpha_{\mathrm{i}}{ }^{*} \cos \left(\varphi-\Theta_{\mathrm{i}}\right) * \mathrm{R}\left(\tau+\mathrm{d}_{\mathrm{i}}\right)\right\} \\
& \mathrm{IE}=\mathrm{A} / 2 * \mathrm{D}^{*}\left\{\cos (\varphi) * \mathrm{R}\left(\tau+\frac{1 / 2_{\text {chip }}}{}\right)+\Sigma_{\mathrm{i}} \alpha_{\mathrm{i}} * \cos \left(\varphi-\Theta_{\mathrm{i}}\right) * \mathrm{R}\left(\tau+\mathrm{d}_{\mathrm{i}}+1 / 2_{\text {chip }}\right)\right\} \\
& \mathrm{IL}=\mathrm{A} / 2 * \mathrm{D} *\left\{\cos (\varphi) * \mathrm{R}\left(\tau-1 / 2_{\text {chip }}\right)+\Sigma_{\mathrm{i}} \alpha_{\mathrm{i}} * \cos \left(\varphi-\Theta_{\mathrm{i}}\right) * \mathrm{R}\left(\tau+\mathrm{d}_{\mathrm{i}}-1 / 2_{\text {chip }}\right)\right\} \\
& \mathrm{Q}=\mathrm{A} / 2{ }^{*} \mathrm{D} *\left\{\sin (\varphi) * \mathrm{R}(\tau)+\Sigma_{\mathrm{i}} \alpha_{\mathrm{i}}{ }^{*} \sin \left(\varphi-\Theta_{\mathrm{i}}\right) * \mathrm{R}\left(\tau+\mathrm{d}_{\mathrm{i}}\right)\right\} \\
& \mathrm{QE}=\mathrm{A} / 2 * \mathrm{D} *\left\{\sin (\varphi) * \mathrm{R}\left(\tau+\frac{1}{2} \mathrm{2}_{\text {chip }}\right)+\Sigma_{\mathrm{i}} \alpha_{\mathrm{i}}^{*} \sin \left(\varphi-\Theta_{\mathrm{i}}\right) * \mathrm{R}\left(\tau+\mathrm{d}_{\mathrm{i}}+1 / 2_{\text {chip }}\right)\right\} \\
& \mathrm{QL}=\mathrm{A} / 2 * \mathrm{D}^{*}\left\{\sin (\varphi) * \mathrm{R}\left(\tau-1 / 2_{\text {chip }}\right)+\Sigma_{\mathrm{i}} \alpha_{\mathrm{i}} * \sin \left(\varphi-\Theta_{\mathrm{i}}\right) * \mathrm{R}\left(\tau+\mathrm{d}_{\mathrm{i}}-1 / 2_{\text {chip }}\right)\right\}
\end{aligned}
$$

The "non-coherent dot-product power" (see Eq. 3.21) is computed:

$$
\mathrm{DF}=\mathrm{I}(\mathrm{IE}-\mathrm{IL})+\mathrm{Q}(\mathrm{QE}-\mathrm{QL})=
$$

$$
\begin{aligned}
& \mathrm{A}^{2} / 4 * \mathrm{D}^{2} *\left\{\cos (\varphi) * \mathrm{R}(\tau)+\Sigma_{\mathrm{i}} \alpha_{\mathrm{i}} \cos \left(\varphi-\Theta_{\mathrm{i}}\right) * \mathrm{R}\left(\tau+\mathrm{d}_{\mathrm{i}}\right)\right\} * \\
& \left\{\cos (\varphi) *\left[R\left(\tau+1 / 2_{\text {chip }}\right)-R\left(\tau-1 / 2_{\text {chip }}\right)\right]+\Sigma_{i} \alpha_{i} \cos \left(\varphi-\Theta_{\mathrm{i}}\right) *\left[R\left(\tau+\mathrm{d}_{\mathrm{i}}+1 / 2_{\text {chip }}\right)-\mathrm{R}\left(\tau+\mathrm{d}_{\mathrm{i}}-1 / 2_{\text {chip }}\right)\right]\right\} \\
& + \\
& \mathrm{A}^{2} / 4 * \mathrm{D}^{2} *\left\{\sin (\varphi) * \mathrm{R}(\tau)+\Sigma_{\mathrm{i}} \alpha_{\mathrm{i}} \sin \left(\varphi-\Theta_{\mathrm{i}}\right) * \mathrm{R}\left(\tau+\mathrm{d}_{\mathrm{i}}\right)\right\} * \\
& \left\{\sin (\varphi) *\left[R\left(\tau+1 / 2_{\text {chip }}\right)-R\left(\tau-1 / 2_{\text {chip }}\right)\right]+\sum_{\mathrm{i}} \alpha_{\mathrm{i}} \sin \left(\varphi-\Theta_{\mathrm{i}}\right) *\left[\mathrm{R}\left(\tau+\mathrm{d}_{\mathrm{i}}+1 / 2_{\text {chip }}\right)-\mathrm{R}\left(\tau+\mathrm{d}_{\mathrm{i}}-1 / 2_{\text {chip }}\right)\right]\right\}
\end{aligned}
$$

The DLL is controlled so that the discriminator output is kept to zero.

Again, the dependency between code and phase loops (and consequently the dependency between code and phase multipath errors) is shown in Eq. 4.15, where $\varphi$ appears and - as demonstrated in $\S 4.1-$ is also biased $\left(\varphi=\varphi_{\mathrm{m}} \neq 0\right)$.

No further simplified expression of the "dot-product power" discrimination function can be derived in the general case of multiple reflected signals. 
In the case of a single reflected signal, Eq. 4.15 becomes:

$\mathrm{DF}=$

$\mathrm{A}^{2} / 4 *\{\cos (\varphi) * \mathrm{R}(\tau)+\alpha \cos (\varphi-\Theta) * \mathrm{R}(\tau+\mathrm{d})\}$ *

$\left\{\cos (\varphi) *\left[R\left(\tau+\frac{1 / 2}{\text { chip }}\right)-\mathrm{R}\left(\tau-1 / 2_{\text {chip }}\right)\right]+\alpha \cos (\varphi-\Theta) *\left[R\left(\tau+\mathrm{d}+1 / 2_{\text {chip }}\right)-\mathrm{R}\left(\tau+\mathrm{d}-1 / 2_{\text {chip }}\right)\right]\right\}$

$+$

$\mathrm{A}^{2} / 4 *\{\sin (\varphi) * \mathrm{R}(\tau)+\alpha \sin (\varphi-\Theta) * \mathrm{R}(\tau+\mathrm{d})\}$ *

$\left\{\sin (\varphi) *\left[R\left(\tau+1 / 2_{\text {chip }}\right)-R\left(\tau-1 / 2_{\text {chip }}\right)\right]+\alpha \sin (\varphi-\Theta) *\left[R\left(\tau+d+1 / 2_{\text {chip }}\right)-R\left(\tau+\mathrm{d}-1 / 2_{\text {chip }}\right)\right]\right\}$

Eq. 4.16 can be developed further and it contains two terms that are similar as the discriminator output given in Eq. 3.21, plus an additional cross-term:

$\mathrm{DF}=$

$\mathrm{A}^{2} / 4 * \mathrm{R}(\tau) *\left[\mathrm{R}\left(\tau+\frac{1 / 2}{2_{\text {chip }}}\right)-\mathrm{R}\left(\tau-1 / 2_{\text {chip }}\right)\right] *\left(\cos ^{2} \varphi+\sin ^{2} \varphi\right)$

$+\alpha^{2 *} \mathrm{~A}^{2} / 4 * \mathrm{R}(\tau+\mathrm{d}) *\left[\mathrm{R}\left(\tau+\mathrm{d}+1 / 2_{\text {chip }}\right)-\mathrm{R}\left(\tau+\mathrm{d}-1 / 2_{\text {chip }}\right)\right]^{*}\left(\cos ^{2}(\varphi-\Theta)+\sin ^{2}(\varphi-\Theta)\right.$

$+\alpha \cos \Theta * A^{2} / 4 *\left\{R(\tau) *\left[R\left(\tau+d+1 / 2_{\text {chip }}\right)-R\left(\tau+d-1 / 2_{\text {chip }}\right)\right]+\left[R\left(\tau+\frac{1 / 2}{\text { chip }}\right)-R\left(\tau-1 / 2_{\text {chip }}\right)\right] * R(\tau+d)\right\}$

Of course, the analytical solution $\tau_{\mathrm{m}}$ of the equation $\mathrm{DF}=0$ is not obvious. But it is possible to show graphically that in the presence of multipath, the zero point of the DF (i.e. the solution of the equation $\mathrm{DF}=0$ ) is shifted and does not correspond to perfect tracking (where the code loop error $\tau$ should be zero).

Fig. 4.3 displays the discriminator output given in Eq. 4.17. Several parameters must be fixed. First the ratio of amplitude (here $\alpha=0.5$ ). Then the delay of the reflected signal ( $d=0.5$ chip). These values were chosen arbitrarily for illustrative purpose. The extrema of phase shift of the reflected signal are chosen $\left(\Theta=0\right.$ or $\left.180^{\circ}\right)$. Note that this discriminator output in the presence of multipath is superimposed to that given in Eq. 3.21 and already displayed in Fig. 3.6. 
$\Theta=0$;

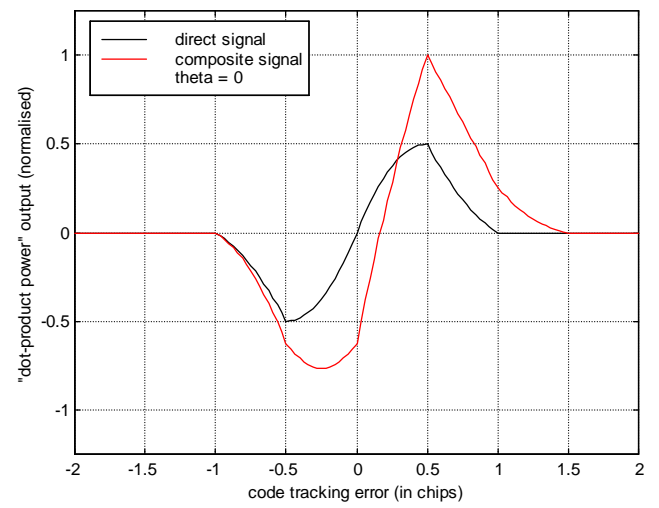

$\Theta=180^{\circ}$

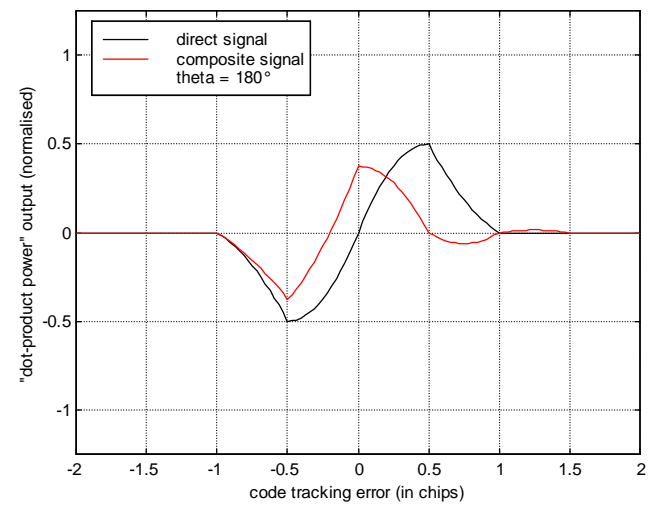

Figure 4.3: deviation of the "non coherent dot-product power" DF in the presence of multipath

Eq. 4.18 introduces the code error envelope corresponding to the usual "coherent early-late envelope" discrimination function. This DF is computed in the presence of multipath, similarly as the "non-coherent dot product power":

$\mathrm{DF}=\mathrm{IE}-\mathrm{IL}=$

$\mathrm{A} / 2 * \mathrm{D} *\left\{\cos (\varphi) * \mathrm{R}\left(\tau+1 / 2_{\text {chip }}\right)+\Sigma_{\mathrm{i}} \alpha_{\mathrm{i}} * \cos \left(\varphi-\Theta_{\mathrm{i}}\right) * \mathrm{R}\left(\tau+\mathrm{d}_{\mathrm{i}}+1 / 2_{\text {chip }}\right)\right\}$

$\mathrm{A} / 2 * \mathrm{D}^{*}\left\{\cos (\varphi) * \mathrm{R}\left(\tau-1 / 2\right.\right.$ chip $\left._{\text {ch }}\right)+\Sigma_{\mathrm{i}} \alpha_{\mathrm{i}}{ }^{*} \cos \left(\varphi-\Theta_{\mathrm{i}}\right) * \mathrm{R}\left(\tau+\mathrm{d}_{\mathrm{i}}-1 / 2\right.$ chip $\left.)\right\}$

The discriminator output in the presence of multipath can be considered as the addition of the individual discriminator outputs corresponding respectively to the direct and reflected signals, each of these discriminator outputs being multiplied by the phase shift cosine term $\cos \left(\varphi-\Theta_{\mathrm{i}}\right)$ :

$\mathrm{DF}=\mathrm{IE}-\mathrm{IL}=$

$\mathrm{A} / 2 * \mathrm{D}^{*}\left\{\cos (\varphi) *[\mathrm{R}(\tau+1 / 2\right.$ chip $)-\mathrm{R}(\tau-1 / 2$ chip $)]+\sum_{\mathrm{i}} \alpha_{\mathrm{i}} * \cos \left(\varphi-\Theta_{\mathrm{i}}\right) *\left[\mathrm{R}\left(\tau+\mathrm{d}_{\mathrm{i}}+1 / 2\right.\right.$ chip $)-\mathrm{R}\left(\tau+\mathrm{d}_{\mathrm{i}}-1 / 2\right.$ chip $\left.\left.)\right]\right\}$

Hence, $\mathrm{DF}=0$ can be solved graphically by a simple addition of the different discriminator outputs. In the presence of multipath, the zero point of the DF (i.e. the 
solution of the equation $\mathrm{DF}=0$ ) is all the more shifted that the phase shift cosine terms will be around 1 or -1 . Considering its envelope (see Fig. 4.2), the multipath phase error $\varphi$ can be approximated to zero, which leads to the maximum positive or negative multipath code errors when $\Theta=0$ or $180^{\circ}$. Note that for these values of the phase shift of the reflected signal, the multipath phase error is effectively theoretically null (see Fig. 4.1), which is coherent with the hypothesis $\varphi \sim 0$. Hence, one must bear in mind that code and phase errors in the presence of multipath always change in quadrature.

Fig. 4.4 assumes a single reflected signal. The corresponding discriminator output is added to that corresponding to the direct signal. The ratio of amplitude and the delay of the reflected signal are again fixed on $(\alpha=0.5)$ and ( $d=0.5$ chip). The two cases $(\Theta=0$ and $180^{\circ}$ ) are displayed. The maximum positive and negative code errors are visible horizontally on this figure. Their magnitude depends on both $\alpha$ and $\mathrm{d}$ (and also the discrimination function).

These multipath code errors are reported vertically in Fig. 4.5, for every delay $\mathrm{d}$ of the reflected signal comprised between 0 and 1.5 chip. This gives the diagram representing the multipath code error envelope (normalised by T), for a fixed ratio of amplitude $\alpha$.

$\Theta=0 ;$

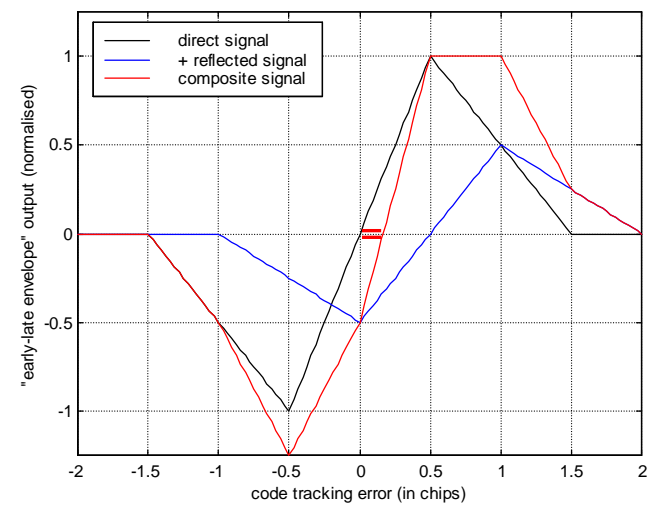

$\Theta=180^{\circ}$

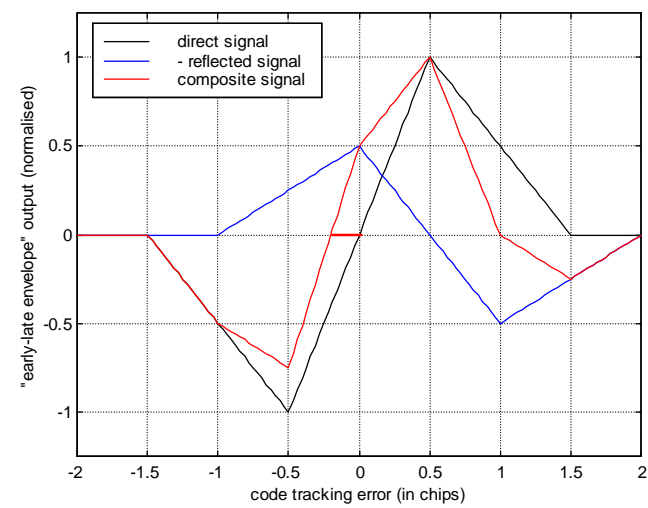

Figure 4.4: deviation of the "coherent early-late envelope" DF in the presence of multipath 
The maximum values of the code error $\tau$ are represented in this diagram, in case of a single reflected signal, with $\alpha=0.5$. Maximum values of $\tau$ vary with the delay $\mathrm{d}$ of the reflected signal. The upper border corresponds to $\Theta=0$ (direct and reflected signals in phase - "constructive" multipath) while the lower border corresponds to $\Theta=180^{\circ}$ (direct and reflected signals in quadrature - "destructive" multipath).

Finally, the next diagram (Fig. 4.5) represents the envelope of code tracking errors $\tau$ for the "coherent early-late envelope" discrimination function, for both C/A and P codes. With this DF, the signals used in the correlator are $1 / 2$ chip early and $1 / 2$ chip late.

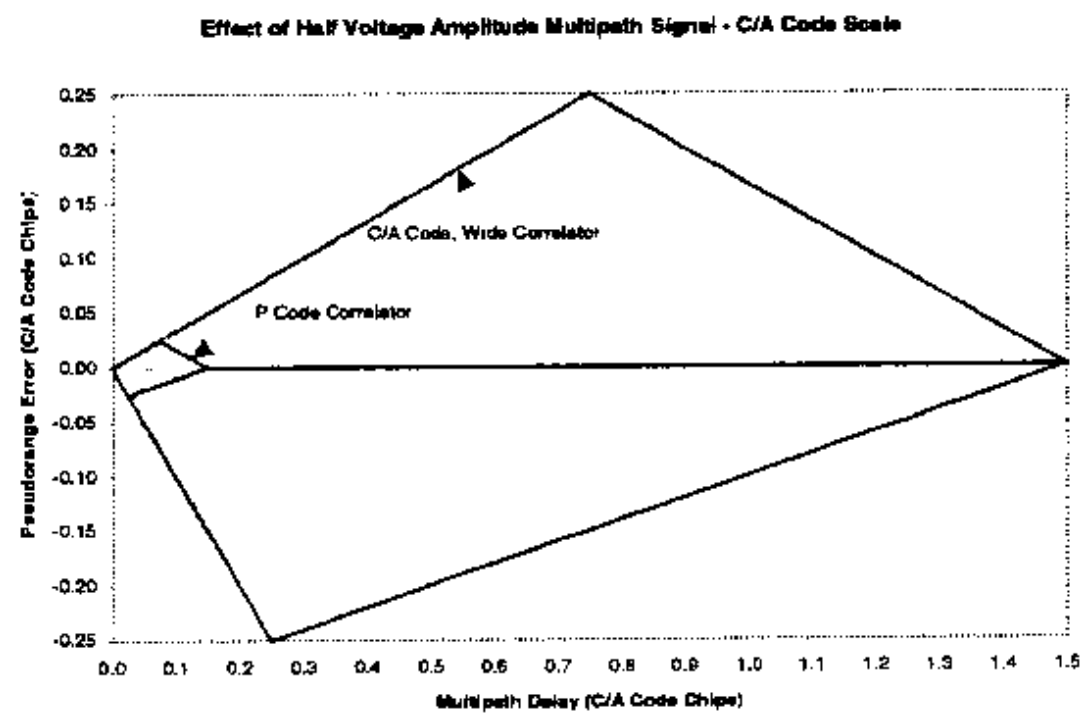

Figure 4.5: maximum C/A and P codes tracking errors in the presence of multipath (source: Leica)

In the presence of multipath, the code error gets biased $\left(\tau=\tau_{\mathrm{m}} \neq 0\right)$, as well as the code measurements that result from hardware counting of the code adjustments.

In addition to maximum values and for further reading, the average and standard deviation values of multipath code errors have been particularly studied by [VAN NEE, 1995]. This thesis showed that the maximum values do not depend on whether the DLL is coherent or non-coherent. On the contrary, the average and standard deviation are different between coherent and non-coherent DLL. It was shown that the average value of 
code errors in time is generally not zero, except in case of rapidly varying multipath and coherent DLL.

\subsection{2 - Case of the "narrow" correlator code tracking}

The first technique that was pointed out in the history of multipath mitigation technique (see Chapter 2, § 2.2) is based on the automatic switch from a "wide" correlator for initial tracking (usually $1 / 2$ chip spacing) to a "narrow" correlator to maintain lock on the signal, whilst reducing the multipath code error. The outline of this process is the following.

- The receiver generates a copy of the GPS code that is moved early by $1 / 2$ chip and late by $1 / 2$ chip: the difference between the early code and the late code (i.e. the wide correlator) is used to correlate the received signal during the initial tracking, while the code tracking error in the loop is "large".

- The early minus late code can then be narrowed (to $10 \%$ e.g.): the narrow correlator is so obtained. This is as efficient as the wide correlator to maintain lock on the signal because the slope ("restoring force") of the discrimination function around zero stays the same (see Fig. 4.6).

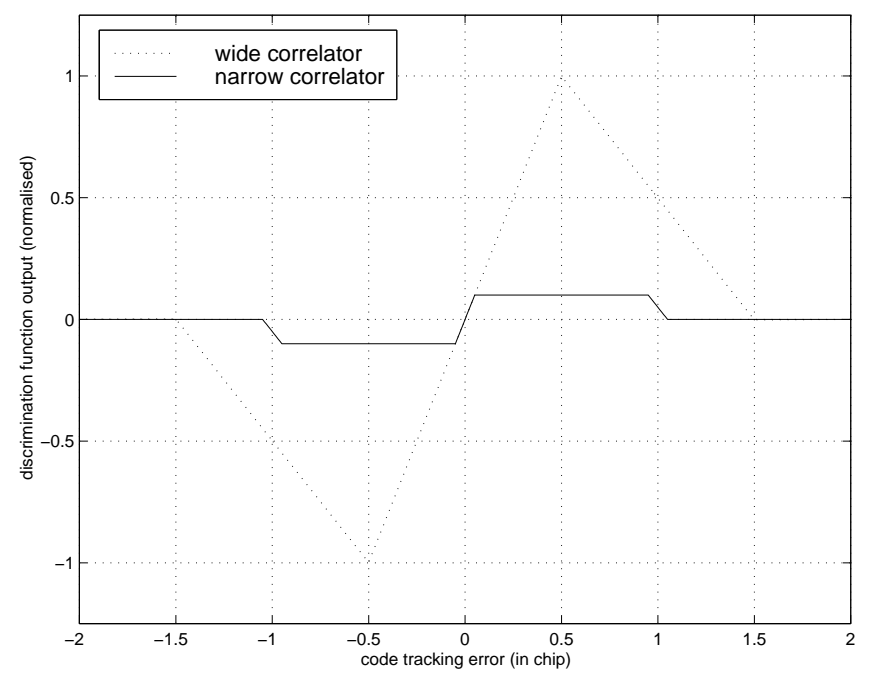

Figure 4.6: wide and narrow "early-late envelope" discrimination functions 
- However, if a "large" code tracking error in the loop was to occur (due to the dynamics of the antenna), it would cause loss of lock and would require initial tracking again. Switching from narrow to wide correlators and vice-versa is automatic in receivers.

- Lastly, in the presence of multipath, the narrow correlator has two advantages with respect to the wide correlator. Not only does it reduce the tracking error, but also makes the tracking non-sensitive to multipath delayed by more than $1+0.5 * 10 \%$ chip (for the $10 \%$ narrow correlator), i.e. 1.05 chip, instead of 1.5 chip (for a wide correlator). A demonstration is not given in this thesis but it can be obtained easily by drawing the same graphical addition as that of Fig. 4.4.

Wide correlators remain sensitive to a certain range of multipath: that causing reflection delays under a 1.05 fraction of the chip length $(\sim 1.05 * 293 \mathrm{~m}$, with respect to $\mathrm{C} / \mathrm{A}$-code, so $\sim 308 \mathrm{~m}$ ). Then possible errors of a maximum 0.025 fraction of the chip length $(\sim 7.5 \mathrm{~m})$ can be generated. Fig. 4.7 represents the envelope of code tracking errors for the "coherent early-late envelope" discrimination function, for C/A-code (signals used in the correlator are ${ }^{1} /{ }_{20}$ chip early and $1 /{ }_{20}$ chip late).

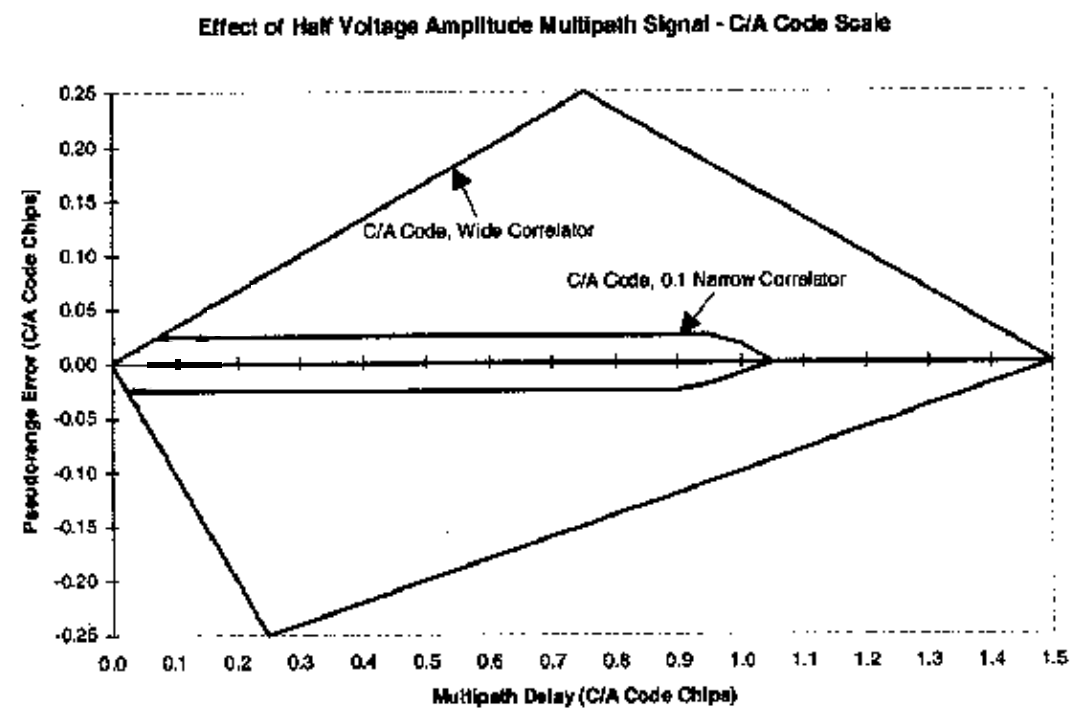

Figure 4.7: maximum C/A-code tracking errors for wide and narrow "early-late envelop" discrimination function (source: Leica) 


\section{3 - Signal-to-noise ratio in the presence of multipath}

\subsection{1 - Definition of the carrier-to-noise and signal-to-noise ratio}

The carrier-to-noise ratio (C/N0) usually characterises the power level of the received signal with respect to the power level of the antenna and receiver ambient noise, in a nominal $1 \mathrm{~Hz}$ bandwidth (it is also expressed in $\mathrm{dB}-\mathrm{Hz}$, instead of $\mathrm{dB}$, which refers to the nominal $1 \mathrm{~Hz}$ bandwidth). $\mathrm{C} / \mathrm{N} 0$ is commonly used at radio and intermediate frequencies.

$\mathrm{c} / \mathrm{n} 0$ expressed as a ratio is usually transformed into $\mathrm{dB}$, by using the relation:

$$
\mathrm{C} / \mathrm{N} 0(\text { in } \mathrm{dB})=10 \log _{10}(\mathrm{c} / \mathrm{n} 0 \text { (ratio) })
$$

The signal-to-noise ratio (SNR) corresponds to the power level of the signal at "base band" in the channel (i.e. at the band it occupies after demodulation of the code) and the power level of the noise in the same band.

SNR is computed from measurements carried out in every channel. Hence it corresponds to a certain satellite, whose signal is processed, and it also depends on the tracking loop used to carry out the measurements. SNR can be derived e.g. from the "I" and “Q" correlator outputs, integrated in the PLL (carrier phase lock-loop).

snr expressed as a ratio is usually transformed into $\mathrm{dB}$, by using the relation:

$\mathrm{SNR}($ in $\mathrm{dB})=10 \log _{10}(\mathrm{snr}$ (ratio) $)$

The relation between SNR and C/N0 depends on the bandwidth in Hz (denoted BW) of the tracking loop (e.g. the bandwidth of the carrier phase lock-loop).

snr $($ ratio $)=(1 / \mathrm{BW}) \mathrm{c} / \mathrm{n} 0$ (ratio)

\subsection{2 - Computation of SNR}

In Leica receivers, the SNR is computed as follows: 
$\mathrm{SNR}=20 \log _{10}\left(\sqrt{ }\left(\mathrm{I}^{2}+\mathrm{Q}^{2}\right) / 2 \mathrm{Q}\right)$

As one can notice, this equation contains a ratio of amplitudes, and this can be rewritten with a ratio of power level as follows: $\mathrm{SNR}=10 \log _{10}\left(\left(\left(\mathrm{I}^{2}+\mathrm{Q}^{2}\right) / 2\right) / 2 \mathrm{Q}^{2}\right)$

$\left(\mathrm{I}^{2}+\mathrm{Q}^{2}\right) / 2$ is the power level of the signal at base band in the channel (this signal being normalised by $\sqrt{2}$ ), whereas $2 \mathrm{Q}^{2}$ is an estimate of the power level of the noise (also normalised by $\sqrt{ } 2$ ). Note that any scale factor normalises both signal and noise and can be ignored in the SNR equation.

Assuming that multipath has essentially an effect on the signal (because of the direct and reflected signals combination) rather than an effect on the noise, the following derivation focuses on the sum of the squared I and Q correlator outputs.

I and $Q$ in steady state are given by Eq. 4.6. They are squared and added in the computation of the signal power $\left(\mathrm{P}=\mathrm{I}^{2}+\mathrm{Q}^{2}\right)$ :

$$
\begin{aligned}
& \mathrm{I}^{2}=\mathrm{P} 0 *\left\{\cos (\varphi) * \mathrm{R}(\tau)+\Sigma_{\mathrm{i}} \alpha_{\mathrm{i}}{ }^{*} \cos \left(\varphi-\Theta_{\mathrm{i}}\right) * \mathrm{R}\left(\tau+\mathrm{d}_{\mathrm{i}}\right)\right\}^{2} \\
& \mathrm{Q}^{2}=\mathrm{P} 0 *\left\{\sin (\varphi) * \mathrm{R}(\tau)+\Sigma_{\mathrm{i}} \alpha_{\mathrm{i}}{ }^{*} \sin \left(\varphi-\Theta_{\mathrm{i}}\right) * \mathrm{R}\left(\tau+\mathrm{d}_{\mathrm{i}}\right)\right\}^{2}
\end{aligned}
$$

$\mathrm{D}^{2}=1$ and disappears. P0 denotes the signal power when there is no multipath.

Although no further simplified expression of the signal power can be derived in the general case of multiple reflected signals, and approximated formula is:

$$
\begin{aligned}
& P=P 0 *\left\{\cos ^{2}(\varphi) * R^{2}(\tau)+2 \cos (\varphi) * R(\tau) * \Sigma_{i} \alpha_{i} \cos \left(\varphi-\Theta_{i}\right) * R\left(\tau+d_{i}\right)\right\} \\
& +P 0 *\left\{\sin ^{2}(\varphi) * R^{2}(\tau)+2 \sin (\varphi) * R(\tau) * \Sigma_{i} \alpha_{i} \sin \left(\varphi-\Theta_{i}\right) * R\left(\tau+d_{i}\right)\right\}
\end{aligned}
$$

where the terms in $\alpha_{i}^{2}$ or $\alpha_{i} \alpha_{j}$ have been neglected, which suppose small values of multipath ratio. 
$P=P 0 *\left\{R^{2}(\tau)+2 R(\tau) * \Sigma_{i} \alpha_{i}\left(\cos (\varphi) \cos \left(\varphi-\Theta_{i}\right)+\sin (\varphi) \sin \left(\varphi-\Theta_{i}\right)\right) * R\left(\tau+d_{i}\right)\right\}$

$\mathrm{P}=\mathrm{P} 0 * \mathrm{R}^{2}(\tau) *\left(1+2 \Sigma_{\mathrm{i}} \alpha_{\mathrm{i}}^{\prime} \cos \left(\Theta_{\mathrm{i}}\right)\right)$

where

$\alpha_{i}^{\prime}$ is the modified ratio of amplitude $\left(\alpha_{i}^{\prime}=\alpha_{i}^{*} R\left(\tau+d_{i}\right) / R(\tau)\right)$ introduced in Eq. 4.8.

Lastly, signal-to-noise ratio and phase error in the presence of multipath always change in quadrature (refer to Eq. 4.9 that links the multipath phase error to $\alpha_{i}^{\prime}$ and $\Theta_{\mathrm{i}}$ ). This is also confirmed graphically in the phasor diagram. When the phase shift of the reflected signal $\Theta=0$ or $180^{\circ}$, the multipath phase error is theoretically null, the code error reaches its envelope (see $\S 4.2 .1$ ), and the composed signal is either maximum (constructive multipath) or minimum (destructive multipath).

\section{4 - Conclusions}

Phase and code multipath error modelling, as well as modelling of the variation of the signal-to-noise ratio in case of multipath, have been presented in this chapter. Their derivation starts from the basic discrimination functions that pilot the loops in ASIC (see Chapter 3). This is a rigorous way to exhibit that these errors and also the SNR variation are tightly linked and dependent.

The modelling of the phase and code errors, as well as that of the signal-to-noise ratio enables several filtering processes that are presented in Chapter 7.

Furthermore, if the environment in the vicinity of the antenna is well known, for instance if it is mounted on the roof of a machinery, the additional path length $L$ travelled by the reflected wave can be determined geometrically, and such modelling (based on the multipath code delay $d$ that equals $\mathrm{L} / \mathrm{c}$ ) can be used to directly correct the code and phase observables. This has been done in the frame of kinematic tests (see Appendix 5). 
Chapter 5

\section{PHASE AND CODE MULTIPATH MITIGATION WINDOW TECHNIQUES}

This chapter presents a comprehensive description of the code and phase multipath mitigation techniques patented by Leica Geosystems ([STANSELL et al., 1996] [HATCH et al., 1997], [HATCH, 2000], [STANSELL et al., 2000]). These techniques are based on the use of a specifically designed "window" of correlation that is further refered to as the “Multipath Mitigation Window” or MMW.

The code multipath mitigation window has already been implemented in the System 500 series of receivers. As far as the phase is concerned, the process presented in the quoted patents is still under development. Different tests were carried out in the frame of this thesis (see Chapter 6).

The various documents that were analysed to write this chapter are listed amongst the references. Most of them are Leica's patents, and contain redundant information. Their claims concern a technique based on MMWs for reducing or eliminating the incidence of multipath signals in the GPS signal code and phase tracking process. Code and phase tracking loops are addressed by the invention (as shown in chapter 4 , multipath has effects on both).

The first and second sections of this chapter examine respectively the MMW process applied on code and phase tracking. The presentation is less formal than in the two preceding chapters: actually, the mathematical derivation given before and the obtained error envelopes remain the same whatever the correlator is, provided that the multipath code delay remains small with respect to the chip duration. 


\section{1- MMW process applied on code tracking}

\subsection{1 - Standard "wide" and "narrow" code correlators}

Chapters 3 and 4 have detailed the functioning of the standard "wide" and "narrow" code correlators. These correlators remain sensitive to a certain range of multipath, depending on the chip spacing in the discrimination function.

With respect to a $1 / 2$ chip early and $1 / 2$ chip late correlator, the maximum multipath delay (that can cause a code tracking error) is reduced from 1.5 to 1.05 chip with the $10 \%$ narrow correlator (i.e. a $1 / 20$ chip early and $1 / 20$ chip late correlator). Moreover, the maximum code tracking error is divided by 10 . Hence, the order of magnitude of the multipath C/A-code error with a narrow correlator is the same as the one used with $\mathrm{P}$-code and a wide correlator (see Fig. 5.1), i.e. a few metres.

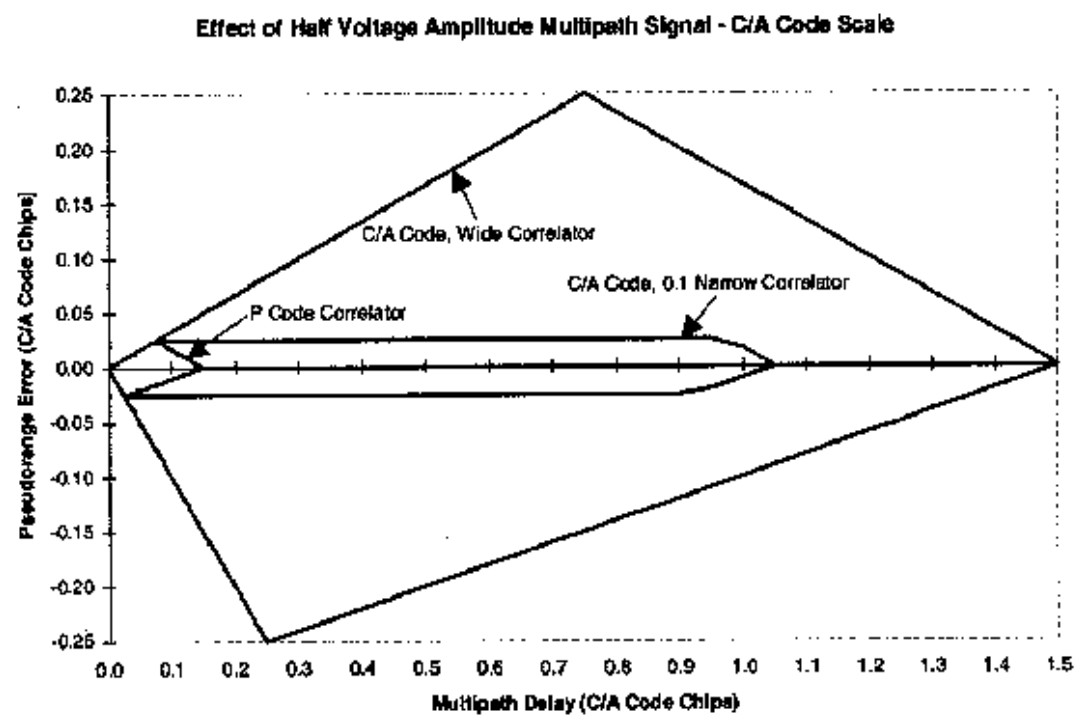

Figure 5.1: maximum $\mathrm{C} / \mathrm{A}$ and $\mathrm{P}$ codes tracking errors for wide and narrow "early-late envelop" discrimination function (source: Leica) 
5.1.2 - Multipath mitigation by "reference waveform" code correlators

\subsubsection{1 - Introduction}

Other signal processing techniques based on the shape of the code correlator have been developed and generally implemented in GPS receivers (see Chapter 2, § 2.2). Leica A and B "Multipath Mitigation Window" correlators [HATCH et al., 1997] are one of these techniques, and they are described further in this chapter.

These correlators are based on the following principle.

- The receiver generates a copy of the GPS code that is moved early by $1 / 2$ chip and late by $1 / 2$ chip: the difference between the early code and the late code (i.e. the wide correlator) is used to correlate the received signal for the initial tracking while the code tracking error in the loop is "large";

- The early minus late code is then replaced by another "reference waveform" (or "Multipath Mitigation Window" in Leica receivers), instead of being only narrowed (see Chapter 4, § 4.2.2). This is as efficient as the wide or the narrow correlator to maintain lock on the signal because the slope of the discrimination function around zero remains the same (see Fig. 5.3 and 5.6).

- Like in the case of a narrow correlator, a "large" code tracking error in the loop would require initial tracking to be repeated. Switching from MMW to wide correlators and viceversa is automatic in receivers.

- In the presence of multipath, the MMW correlator has the same advantage as the equivalent narrow correlator with respect to the tracking error reduction. But it additionally makes the tracking non-sensitive to multipath delayed by more than a certain fraction of the window size (for example, 0.15 chip for the MMW equivalent to the $10 \%$ narrow correlator, instead of 1.05 chip). This is due to the shape of the reference waveform, which entails a particular feature with respect to the code correlation, as explained graphically on the following figures. 


\subsubsection{2 - Generation of the Multipath Mitigation Window}

Further in Fig. 5.3 and 5.6, the Multipath Mitigation Window has been obtained by the following combination of three different modulations (named "first", "central" and "last" modulations) of a $1 / 8^{\text {th }}$ chip early shifted GPS signal replica, as shown in Fig. 5.2:

- the $1^{\text {st }}$ earliest $1 / 16^{\text {th }}$ of a $1 / 8^{\text {th }}$ chip early shifted code replica

+ the $2^{\text {nd }}$ and $3^{\text {rd }}$ earliest $1 / 16^{\text {th }}$ of a $1 / 8^{\text {th }}$ chip early shifted code replica

- the $4^{\text {th }}$ earliest $1 / 16^{\text {th }}$ of a $1 / 8^{\text {th }}$ chip early shifted code replica

$$
\text { = the MMW correlating signal (reference waveform) }
$$

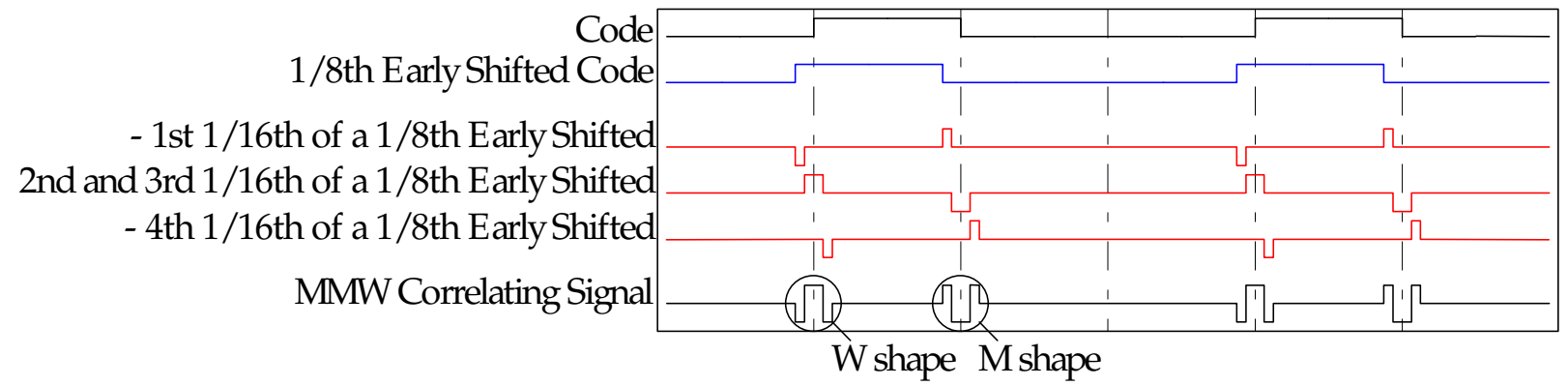

Figure 5.2: generation of the reference waveform in a MMW correlator

Note that a "W shape" MMW window corresponds to 0-to-1 code transitions and an inverse polarity (i.e. inverse sign) "M shape" $\mathrm{MMW}$ window corresponds to 1-to-0 code transitions.

\subsubsection{3 - Correlation of the signal with the Multipath Mitigation Window (type A)}

In Fig. 5.3, the $1^{\text {st }}$ and $2^{\text {nd }}$ lines (black) show the code replica and the MMW type A correlating signal generated by the receiver. Fig. 5.4 shows the MMW type A discrimination function. 


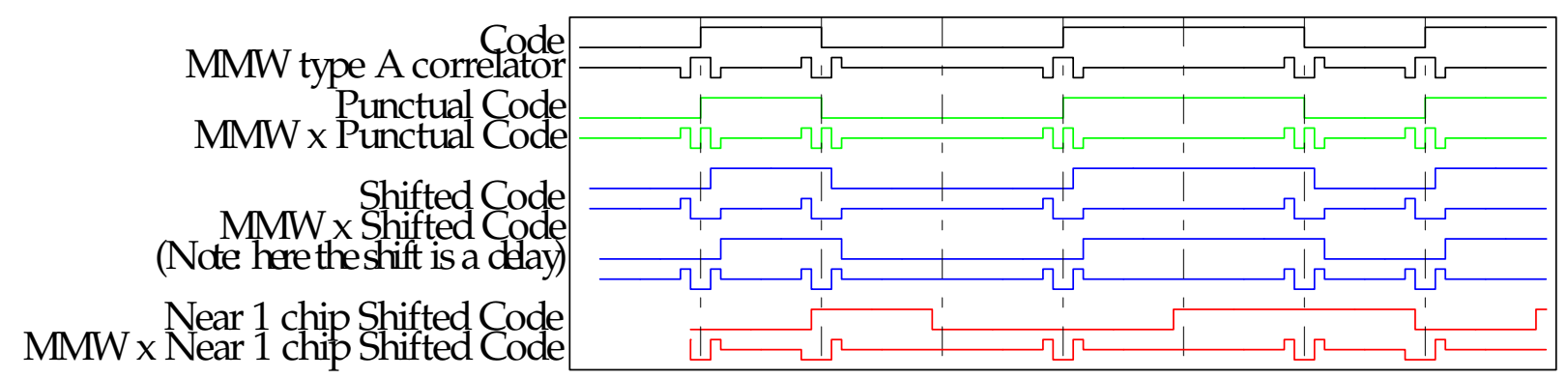

Figure 5.3: MMW type A correlation process

If the received signal is "code-punctual", then the average value of this code multiplied by the MMW correlating signal is zero ( $3^{\text {rd }}$ and $4^{\text {th }}$ lines (green)). Next lines ( $5^{\text {th }}$ and $6^{\text {th }}$ (blue)) show a received signal "code-shifted" (a delay or an advance would be equivalent since the MMW is symmetric), with the corresponding correlation, which is NOT zero: this enables code tracking. When the code shift for lines $7^{\text {th }}$ and $8^{\text {th }}$ is equal to half the MMW size, then the correlation is back to zero, because the overall "surface" of the MMW is zero (the window contains the same number of 1 and -1 occurrences: it has an average value of zero). This is what happens to multipath signals, delayed by half the MMW size or more. However, the $9^{\text {th }}$ and $10^{\text {th }}$ (red) last lines show a quasi-one chip shifted code and its correlation by MMW. A non-zero average value is output, so multipath errors around one chip delayed reflected signals are possible! This issue of type A is addressed in §5.1.2.4.

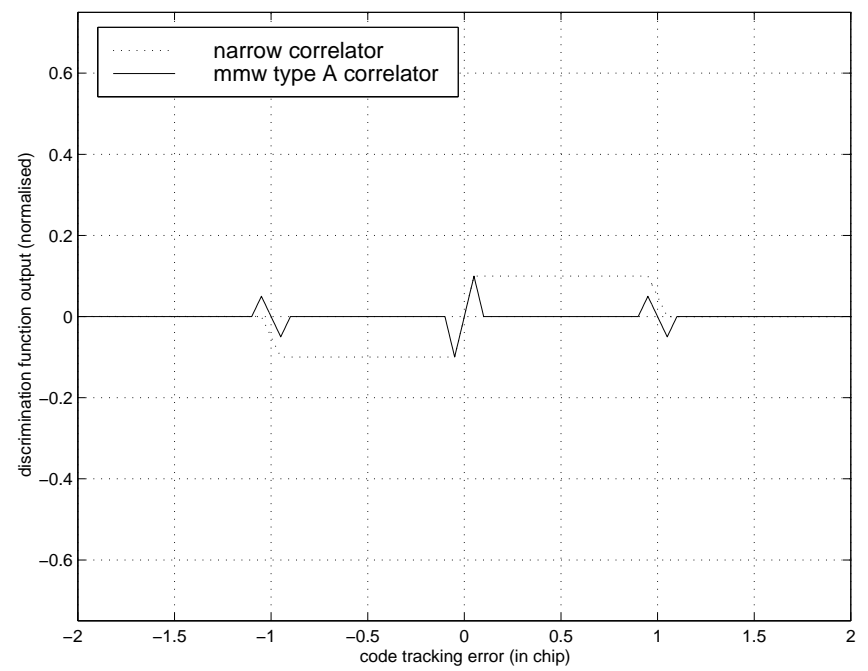

Figure 5.4: MMW type A discrimination function 
The next diagram (Fig. 5.5) represents the envelope of code tracking errors for the Leica MMW type A discrimination function, for C/A-code, with the assumption that the multipath ratio of amplitude equals 0.5 . This envelope has three parts. The last two parts correspond to the multipath errors caused by around one chip delayed reflected signals.

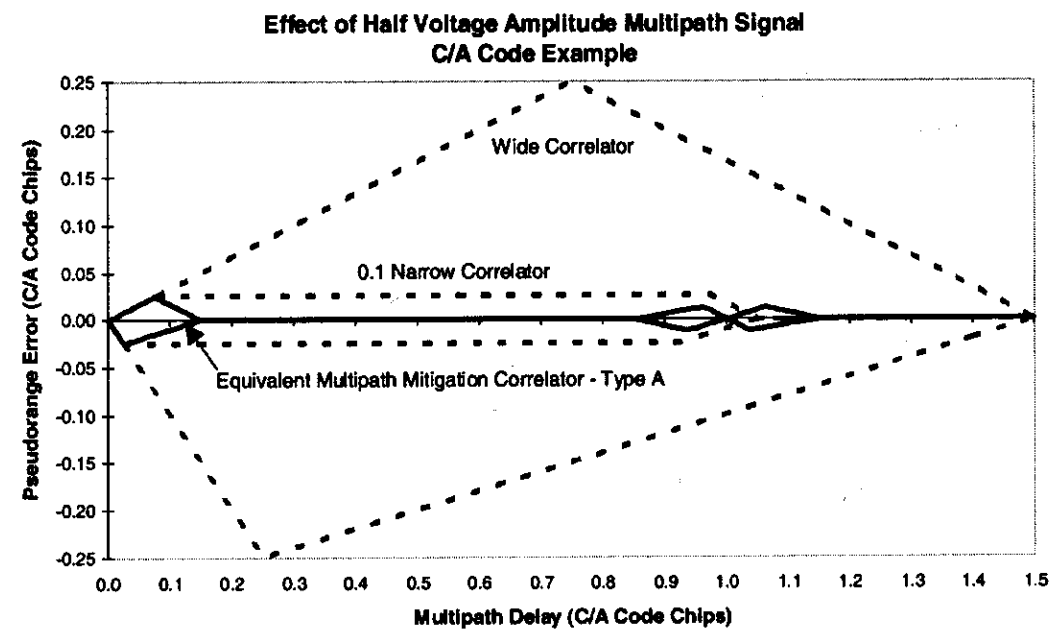

Figure 5.5: maximum C/A-code tracking errors for MMW type A discrimination function (Leica)

\subsubsection{4 - Correlation of the signal with the Multipath Mitigation Window (type B)}

In Fig. 5.6, the $1^{\text {st }}$ and $2^{\text {nd }}$ lines (black) show the code replica and the MMW type B correlating signal generated by the receiver. Contrary to type A, the MMW window is repeated in a certain way at every code clock in type B, and not only at every code transition. Because the surface of the MMW is zero, it does not contribute to the correlation output unless a code transition occurs within its boundaries. The progress is that, if the received signal is around one chip delayed, a zero average value is now output with such a correlator. This is due to the equal distribution of transitions and nontransitions in a PRN code. For example, 0-to-1 transitions in the one chip delayed code are correlated:

- either with a "M shape" window (if there is a 1-to-0 transition following);

- either with a "W shape" window (if there is no transition following). 
Both cases have the same occurrence and cancel in the correlation output. This keeps true for 1-to-0 transitions in the one chip delayed code.

However, if the received signal is around one chip advanced, the above demonstration fails. So, type B solves the type A problem when the code is one chip delayed.

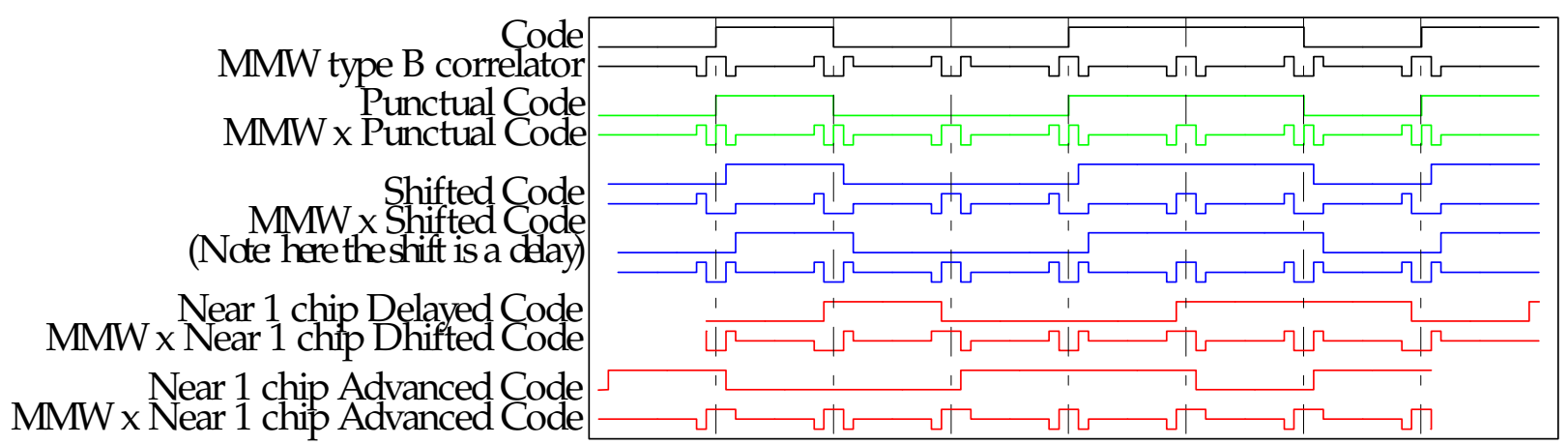

Figure 5.6: MMW type B correlation process

Fig. 5.7 shows the MMW type B discrimination function.

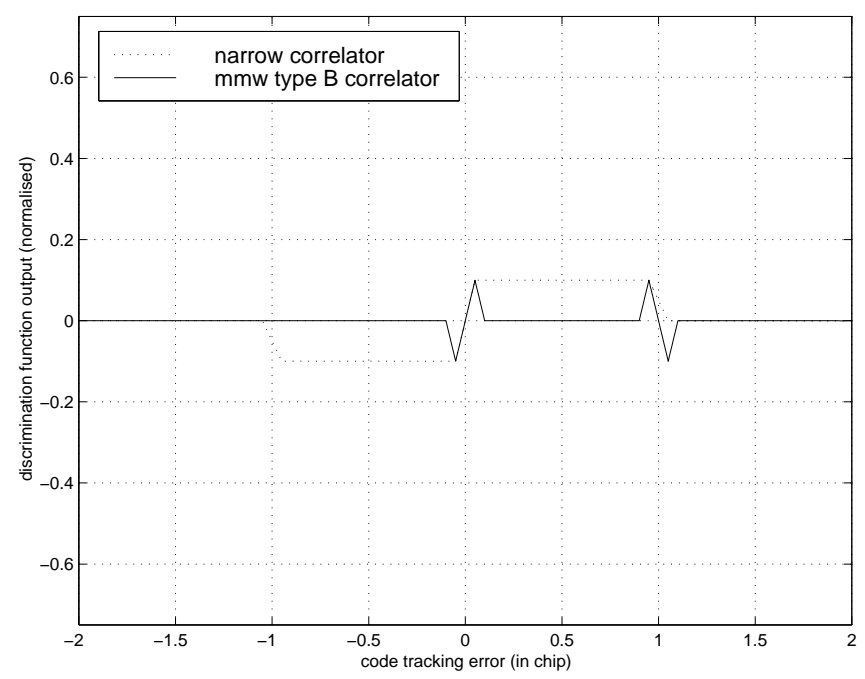

Figure 5.7: MMW type B discrimination function

The next diagram (Fig. 5.8) represents the envelope of code tracking errors for the Leica MMW type B discrimination function, for C/A-code, with the assumption that the 
multipath ratio of amplitude equals 0.5. The first part of the envelope is the same as type A, and multipath errors are no longer caused by around one chip delayed reflected signals.

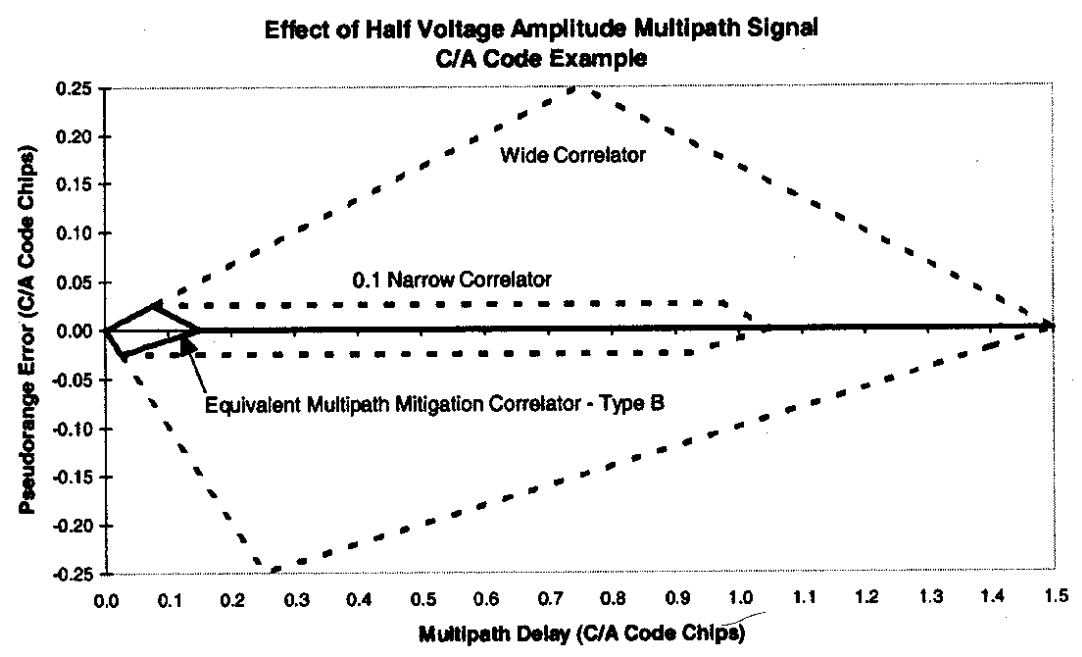

Figure 5.8: maximum C/A-code tracking errors for MMW type B discrimination function (Leica)

\subsubsection{5 - Asymmetric code Multipath Mitigation Window}

Because of the additional windows at code clock with no transition, the noise in type B correlator is twice that in type A, whereas the signal content is the same. Windows that correlate non-transitions of the received signal cause the signal-to-noise ratio to decrease by $3 \mathrm{~dB}$ (i.e. $\left.10 * \log _{10}(1 / 2)\right)$.

Since multipath signals are always delayed relatively to direct signal, the first modulation of the MMW can be suppressed with no consequence on the code error envelope. Only the discrimination function is modified, see Fig. 5.9, in its half side that concerns advanced signals. The overall surface of this asymmetric MMW window is reduced by $25 \%$, as well as the noise induced.

Note: the average value of the punctual code multiplied by the asymmetric MMW correlating signal is zero because transitions and non-transitions compensate, since they are the same number in PRN codes. The asymmetric MMW needs to be repeated at every code 
clock, otherwise the average value when correlating the punctual code would not be zero, but biased. So, the asymmetric MMW is always type B and never type A.

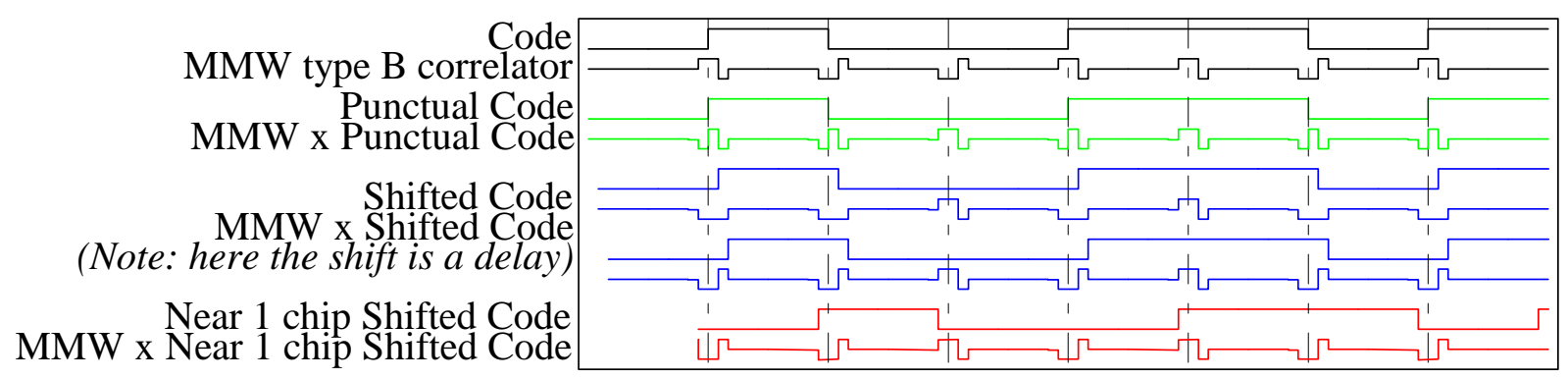

Figure 5.9: asymmetric MMW type B correlation process

Fig. 5.10 shows the asymmetric MMW type B discrimination function.

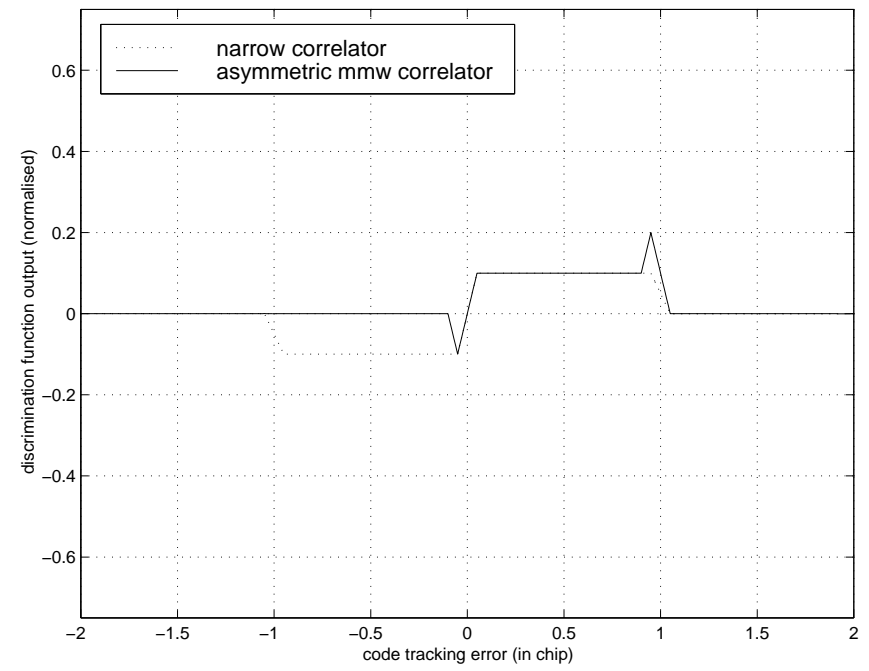

Figure 5.10: asymmetric MMW type B discrimination function

The envelope of code tracking errors for the Leica asymmetric MMW discrimination function is the same as type B (see Fig. 5.8).

\subsubsection{6- Conclusions}

To conclude, the technique shows that an "infinite" variety of MMWs is possible, by changing both parameters: duration in time and amplitude of the different modulations of 
the MMWs. The interest of suppressing the first modulation has also been demonstrated. Finally, the basic requirement that needs to be met concerns the half surface of the MMWs (i.e. the overall surface after the code clock): it must be zero. If not, as shown for example in Fig. 5.11 where the amplitude of the central modulation has been doubled, one can notice that the average value when correlating the punctual code is not zero.

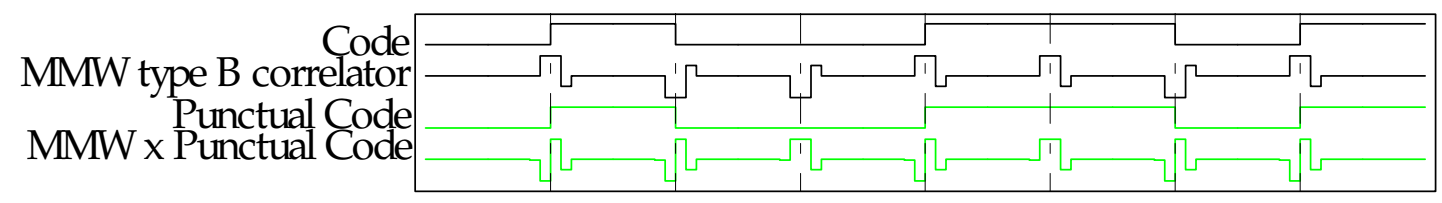

Figure 5.11: incorrect asymmetric MMW correlation process (the overall surface of the second half part of the MMW is not zero)

Fig. 5.12 shows the discrimination function corresponding to the example of an incorrect asymmetric MMW depicted in Fig. 5.11. One can notice it is biased around zero!

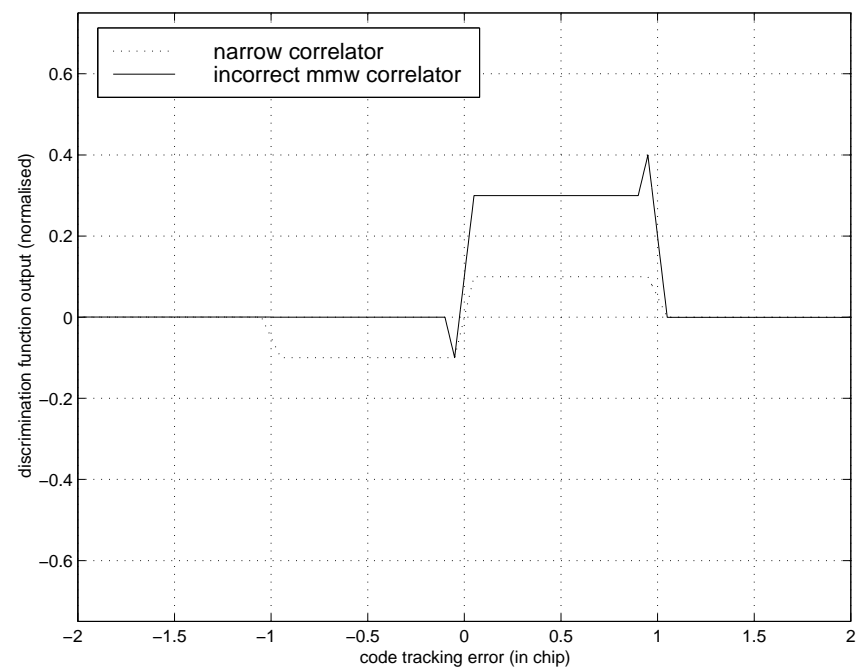

Figure 5.12: incorrect asymmetric MMW discrimination function (the overall surface of the second half part of the MMW is not zero)

The different discrimination functions (Fig. 5.4, 5.7 and 5.10) and diagrams of code error envelope (Fig. 5.5 and 5.8) correspond to MMWs equivalent to the $10 \%$ narrow correlator. This means that the duration in time of the central modulation is two times $1 / 20^{\text {th }}$ chip (i.e. $1 / 20^{\text {th }}$ chip or approximately $50 \mathrm{~ns}$ at both sides of the code clock). 
The following schemes represent different multipath mitigation windows. Their corresponding multipath error envelopes are given in Fig. 5.13 (for a multipath ratio of amplitude $\alpha=0.5$ ) with the following colours:

-blue: the wide correlator (the chip spacing is $\pm 1 / 2$ chip)

- red: the $10 \%$ narrow correlator (the chip spacing is $\pm 1 / 20$ chip)

- black: the $10 \%$ equivalent asymmetric MMW at every code clock with the last modulation half large (in duration)

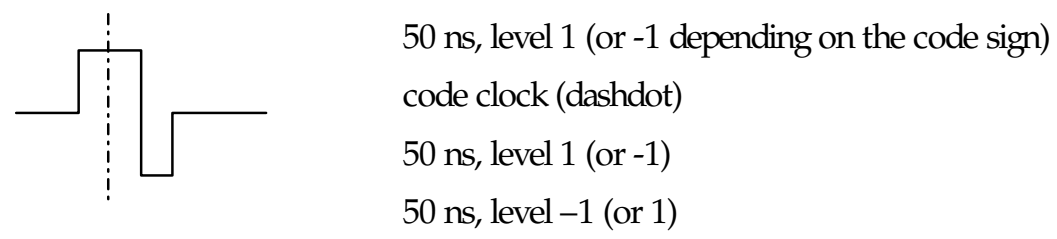

- pink: the $10 \%$ equivalent asymmetric MMW at every code clock with the last modulation half high (in amplitude)

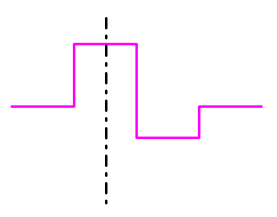

$50 \mathrm{~ns}$, level 1 (or -1 depending on the code sign)

code clock (dashdot)

50 ns, level 1 (or -1 )

$100 \mathrm{~ns}$, level $-1 / 2$ (or $1 / 2$ )

The size of the modulations can be reduced again. In the following scheme, it is twice as small. As shown on Fig. 5.13, the corresponding code error envelope is also twice as small.

- green: the $5 \%$ equivalent asymmetric MMW at every code clock with the last modulation half high (in amplitude)
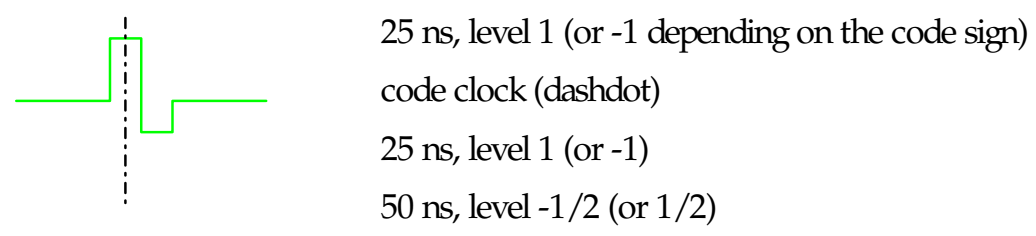

Note that the error envelopes are the same around zero (i.e. for small multipath code delays), which corresponds to the same slope (or "restoring force") of the different DFs.

Lastly, a practical limitation exists in reducing the size of the modulations of the MMWs, due to the bandwidth of the signal, as explained in the next section (§ 5.1.2.7). 


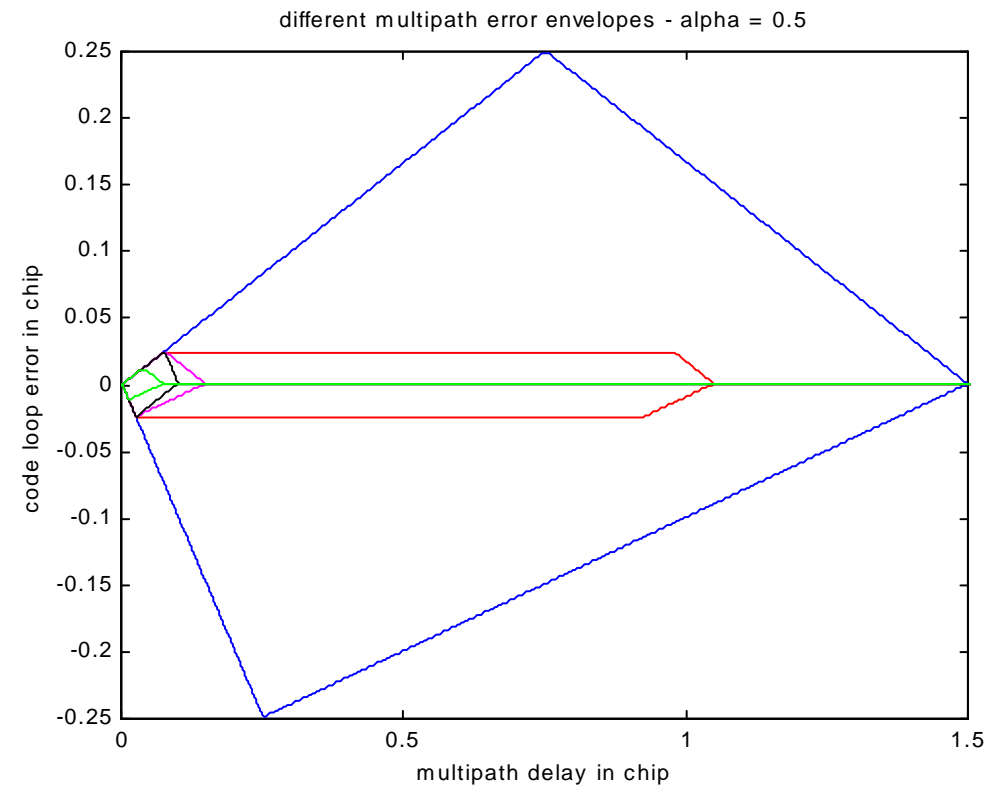

Figure 5.13: maximum C/A-code tracking errors for the different correlators listed above

\subsection{3 - Implementation of the code MMW}

\subsubsection{1 - Received signal bandwidth}

As far as the implementation of the code Multipath Mitigation Window is concerned, the duration in time of the different modulations is constrained by the bandwidth of the signal that enters the code loop. This bandwidth results from both the satellite signal emitting bandwidth and the bandwidth of the signal conditioning in the antenna and the receiver before entering the ASIC. So the signal that is processed in the correlators is not infinite bandwidth. In other words, the code transitions are not instantaneous. In Leica receivers, one can consider that they last for about $40 \mathrm{~ns}$.

There is a trade-off between the duration of the code transition and the capability of the MMW to mitigate multipath. In other words, with a smaller size of MMW modulations, the code error envelope is theoretically reduced, but because of the time needed for the code transition to take place, the signal-to-noise ratio decreases dramatically in the correlation process, and in the output code measurements. 


\subsubsection{2 - Code MMW sampling strategy}

It is important to bear in mind that all the process described before is in discrete and not continuous time. Thus, its digital implementation consists in sampling the received signal at certain instants and with certain amplitudes, in accordance with the designed MMW. Practically, the actual implementation is based on the Leica receivers clock rate of approximately $40 \mathrm{MHz}$ (exactly 4.25 * $10.23 \mathrm{MHz}$, see Chapter 3, § 3.2.2): one sample can be taken at every $25 \mathrm{~ns}$.

The code MMW that is implemented in Leica receivers uses only two samples, one at each side of every code clock: one sample at level 1 (or -1 depending on the code sign) followed by one sample at level $-1 / 2$ (or $1 / 2$ ). This strategy effectively works because the positions of the MMW samples relative to the received code clocks (either transitions or not) are constantly changing in time (if not, that would assume zero Doppler and zero oscillator frequency error, but both of these latter assumptions are not the norm). Because of Doppler and also a certain instability of the oscillator, the received and generated codes shift respectively to each other. And the capability of the code generator to keep synchronised with the received code is limited by the resolution of the receiver clock (1 sample every $25 \mathrm{~ns}$ ). In fact, the actual MMW code loop controls the code transitions to occur exactly between the two clocks of the two MMW samples on the average, as shown on Fig. 5.14. The transitions are never synchronised to the actual samples, but slide between the clock edges (refer to the end of Chapter 3, § 3.4, about the sampling strategy).

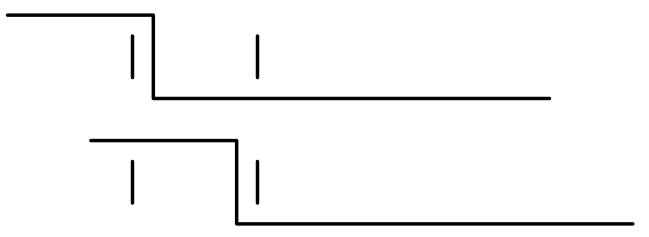

Figure 5.14: MMW sampling 
The smallest phase error envelope in green on Fig. 5.13 has been obtained in a numerical simulation by making two hypotheses: an infinite signal bandwidth and a signal sampling at a frequency high enough to enable at least 10 samples to be taken within the 25 ns duration of each MMW modulation.

This envelope corresponds to a 100 ns duration MMW. Its dimensions (for a multipath ratio of amplitude $\alpha=0.5$ ) are:

0.075 chip large (multipath delay $<22 \mathrm{~m}$ );

0.0125 chip high (code loop error $<3.75 \mathrm{~m}$ ), for $\mathrm{C} / \mathrm{A}$-code.

These are correct but the time scale is twice as long as it should be in reality, with the actual code MMW implementation, which is actually equivalent to the $2.5 \%$ narrow correlator.

Finally, for a multipath amplitude ratio $\alpha=0.5$, the dimensions of the smallest envelope that corresponds to Leica code MMW implementation are:

0.0375 chip large (multipath delay $<11 \mathrm{~m}$ );

0.00625 chip high (code loop error $<2 \mathrm{~m}$ ), for C/A-code.

\subsection{4 - Conclusions relating to the Code Multipath Mitigation Window process}

Both the $10 \%$ narrow correlator and its equivalent Multipath Mitigation Window correlator may generate possible errors of maximum 0.025 fraction of the chip length $(\sim 0.025 * 293 \mathrm{~m}$, with regard to C/A-code, so $\sim 7.5 \mathrm{~m})$ for a multipath ratio of amplitude $\alpha=0.5$. But these errors are completely mitigated for multipath delays above 0.15 fraction of the chip length $(\sim 44 \mathrm{~m})$, with the code MMW correlator, instead of 1.05 fraction of the chip length $(\sim 300 \mathrm{~m})$ with the $10 \%$ narrow correlator.

The actual implementation of the code MMW in Leica receivers enables reducing the multipath code error envelope to approximately $10 \mathrm{~m}$ in terms of multipath delay and $2 \mathrm{~m}$ in terms of error amplitude. 


\section{2 - MMW process applied to phase tracking}

\subsection{1 - Introduction}

The multipath mitigation technique based on the use of reference waveform code correlators has proved its efficiency on code measurements, both theoretically as demonstrated in $\S 5.1$ and practically. Most manufacturers have now implemented this technique in their last receivers. But it does not address directly the effect of multipath on phase measurements.

[STANSELL et al., 2000] contains an extension of this technique to phase tracking that is based again on Multipath Mitigation Windows (MMWs). A description of this technique is given below.

The phase MMW will be introduced by means of phasor diagrams.

\subsection{2 - Recall of the meaning of the phasor diagram in the presence of multipath}

Firstly, let us recall the way in which a standard phase loop would work in a situation where a reflected signal is superimposed on the direct one.

Fig. 5.15 shows a portion of a direct signal with a single code transition and the same code transition, delayed by $\mathrm{d}=\mathrm{L} / \mathrm{c}$ for a reflected signal ( $\mathrm{L}$ is the additional path length). These portions are 2 chips long, and the data bit is supposed to keep unchanged in the meanwhile. Note that in the figure the phase shift $\Theta$ between the direct and the reflected signals exactly corresponds to the additional path length travelled by the reflected signal divided by the wavelength +180 degrees. In practice, a change of the additional distance of a few centimetres, which is negligible with respect to the chip length, causes a significant change of the phase shift of the reflected signal. This cannot be shown in the figure, where - for illustrative purpose - the frequency of the represented carrier is only 10 times the chip rate. In Fig. 5.15, the 180 degrees phase shift is supposed to result from the addition of the reflection phase shift and the antenna phase shift that respectively depends on the material of the reflector and the antenna phase pattern, as already mentioned in Chapter 1. 
clock

$\mathrm{C}(\mathrm{t})$

$\operatorname{direct}=\mathrm{AC}(\mathrm{t}) \sin (\mathrm{wt})$

$\mathrm{C}(\mathrm{t}-\mathrm{d})$

reflected $=\& A C(t-d) \sin (w t-\varnothing)$

$\operatorname{IP}(\mathrm{t})=\mathrm{C}(\mathrm{t}) \sin (\mathrm{wt})$

$\mathrm{QP}(\mathrm{t})=\mathrm{C}(\mathrm{t}) \cos (\mathrm{wt})$

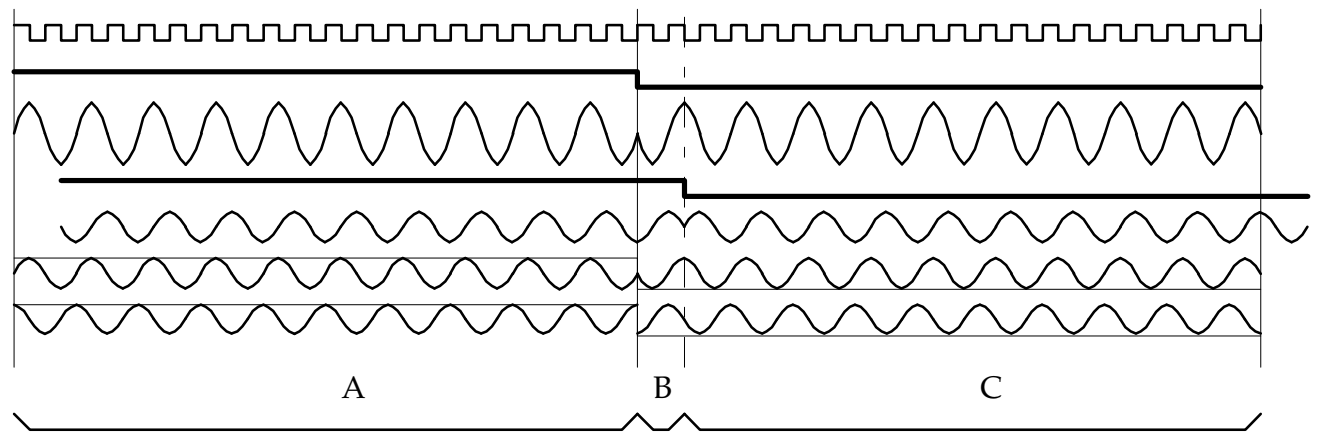

Figure 5.15: direct and multipath signals entering the standard phase loop

The phasor diagram representation of the signals (see Eq. 3.13) uses the vertical axis for the "I" product (product of the signal with a code prompt replica in phase: IP) and the horizontal axis for the " $\mathrm{Q}$ " product (product of the signal with a code prompt replica in quadrature: QP).

The tracking loop is driven by the average of the I and $Q$ values in time. The different components corresponding to the different signals that are present (direct and reflected) are added. The resulting vector is that entering the loop. The loop is closed so that the $\mathrm{Q}$ component of the resulting vector equals zero.

Fig. 5.16 shows the vectors $\mathrm{A}, \mathrm{B}$ and $\mathrm{C}$ corresponding to the time interval $\mathrm{A}, \mathrm{B}$ and $\mathrm{C}$ in Fig. 5.15:

- A is an interval before a direct code transition;

$\mathrm{A}=\mathrm{D}+\mathrm{M}$, with $\mathrm{D}$ for direct component and $\mathrm{M}$ for multipath component.

- $\mathrm{B}$ in just after $\mathrm{A}$ and before the multipath code transition;

$\mathrm{B}=\mathrm{D}-\mathrm{M}$. Compared to the first interval, $\mathrm{M}$ has changed to $-\mathrm{M}$, since the polarity of the replica has changed (along with that of the direct signal), while the polarity of the reflected signal remains unchanged.

- $C$ is just after $B$ until the next direct code transition.

$\mathrm{C}=\mathrm{D}+\mathrm{M}=\mathrm{A}$. Compared to the second interval, $-\mathrm{M}$ has changed to $\mathrm{M}$. The polarity of the reflected signal has changed, and it is now the same as that of the replica. 


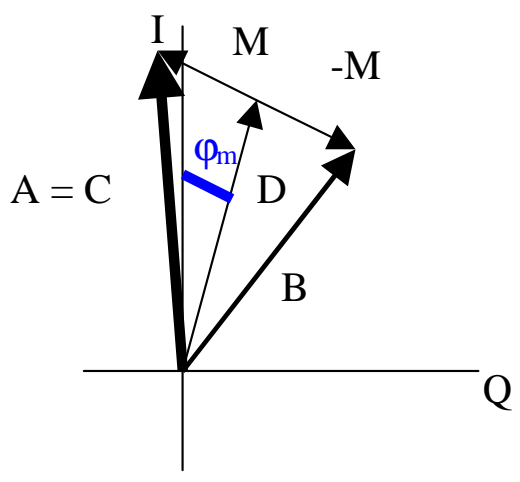

Figure 5.16: phasor diagram of direct and multipath signals corresponding to the standard phase loop correlator

The standard phase loop averages continuously in time, which corresponds here (on the 2 chips duration of the example) to the summation of:

-A (during the first 40 samples);

- then B (during the next 3 samples);

- and finally $\mathrm{A}$ again, since $\mathrm{C}$ is equal to $\mathrm{A}$ (during the last 37 samples).

The ratio $3 /(40+37)$ on the example given in the preceding figures stays the same when integrating over the PIT, and it explains why the vector A and the vertical I axis corresponding to the tracking loop closure are not aligned. The I axis is slightly biased away from vector A toward vector $\mathrm{B}$. The misalignment has the same proportion as the ratio of intervals A and B when integrating over time.

Moreover, the I axis is quite far from the vector $\mathrm{D}$, which corresponds to the direct signal only. The alignment of the tracking loop onto the vector $\mathrm{D}$ would in fact produce the desired phase measurement, multipath free.

To conclude, the standard phase loop shows a bias ( $\varphi_{\mathrm{m}}$ or the multipath phase error) in case of multipath. It can also be shown graphically that the more the code is delayed, the less the tracking loop will be biased. It is completely unbiased if the code is delayed by one chip or more (see Chapter 4, Fig. 4.2). 


\subsection{3 - The phase MMW correlator}

As has been mentioned in the introduction of this chapter, the MMW correlator has been introduced through a number of patents, e.g. [STANSELL et al., 2000]. It is essentially a phase MMW sampler that has a short polarised component before the direct signal code transition (interval A on Fig. 5.17) and followed by another short opposite component (interval B on Fig. 5.17). This second component immediately follows the direct signal code transition, but (so long as the reflected signal is sufficiently delayed) ends before the reflected signal code transition (dashdot line on Fig. 5.17). The polarity of these components is determined by the polarity of the code at the same time, exactly in the same way as in a standard phase correlator.

Fig. 5.15 is here modified as suggested by the patents. In Fig. 5.17, the polarity of the different components is positive and then negative due to the fact that the code transition is - for example - locally positive to negative.

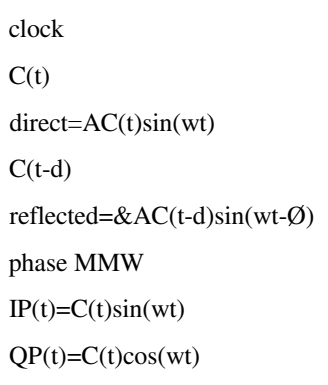

$\operatorname{direct}=\mathrm{AC}(\mathrm{t}) \sin (\mathrm{wt})$

$\mathrm{C}(\mathrm{t}-\mathrm{d})$

phase MMW

$\mathrm{QP}(\mathrm{t})=\mathrm{C}(\mathrm{t}) \cos (\mathrm{wt})$

Figure 5.17: direct and multipath signals entering the MMW phase loop

Vectors A and B are displayed using a phasor diagram representation. A and B are respectively the composite vectors corresponding to the first and second intervals of the phase MMW sampler. $\mathrm{A}=\mathrm{D}+\mathrm{M}$, and $\mathrm{B}=\mathrm{D}-\mathrm{M}$. There is no interval C. 

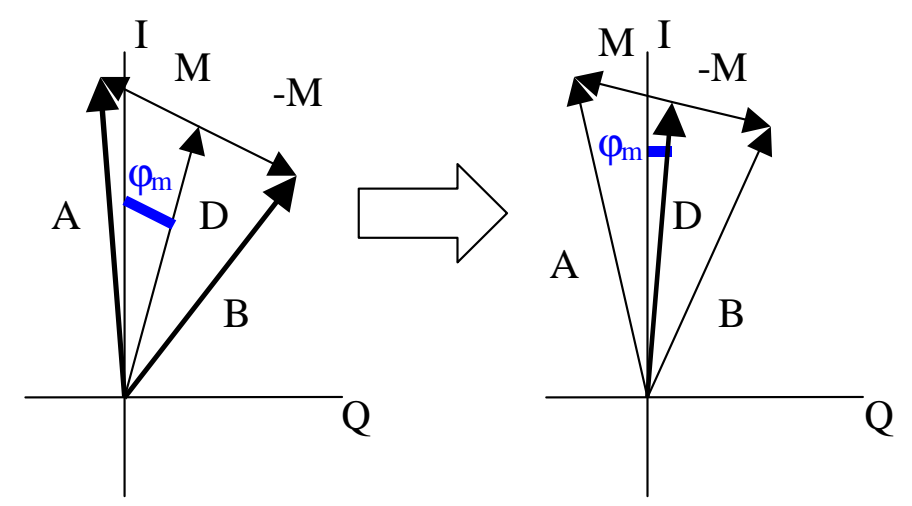

Figure 5.18: phasor diagram of direct and multipath signals corresponding to the MMW phase loop correlator

The invention relies on the key observation from Fig. 5.18 that the vector average of vectors $A$ and $B$ is vector $D$, which corresponds to the direct signal without any multipath distortion. If vectors A and B have the same "weight" in the integration carried out by the tracking loop, then the tracking loop will be aligned on vector $\mathrm{D}\left(\varphi_{\mathrm{m}}\right.$ becomes zero).

Therefore, instead of integrating continuously as in a standard phase loop, the MMW technique integrates only during the MMW samples. The technique is neither limited by the number of reflected signals nor by their amplitude.

\subsection{4 - Implementation of the phase MMW}

\subsubsection{1 - Received signal bandwidth}

The MMW correlator works provided that the occurrence of the multipath signal transitions are sufficiently delayed with respect to the direct code transitions, for the measurement samples to be taken. In other words, the sooner a sample is taken in the received signal after a code transition, the better.

Hence, the bandwidth of the received signal that enters the phase loop is of great importance. This bandwidth is around $25 \mathrm{MHz}$, which means that a code transition will last for some $40 \mathrm{~ns}$. Consequently, an equivalent delay must occur before taking a sample in the received signal. With a clock rate of $40 \mathrm{MHz}$, the first sample comes after a delay of $25 \mathrm{~ns}$ 
by which time the code transition is not quite complete. It is, however, considered to be just sufficient with regard to the bandwidth of the signal.

$40 \mathrm{MHz}$ (which corresponds to an additional path length of approximately $7.5 \mathrm{~m}$ ) is therefore a good trade-off between the duration of the code transition and the capability of the MMW to mitigate multipath. Increasing this rate would enable multipath with shorter delays (i.e. from closer reflectors) to be mitigated but would lead to an increase in noise due to use of a less complete code transition.

Consequently, only reflected signals with an additional path length over $7.5 \mathrm{~m}$ will be theoretically eliminated in total, while reflected signals with shorter path lengths will be mitigated to a lesser extent.

\subsubsection{2 - Asymmetric phase Multipath Mitigation Window}

A second implementation issue to be mentioned concerns where the samples should be taken. What the technique suggests is to take the samples before and immediately after every code transitions. The case of a multipath with a delay of around one chip must be considered (see dashdot line in Fig. 5.19). Two possible cases shown in this figure need to be examined: either the preceding chip has the same polarity (blue) as the current chip, either the opposite (red).

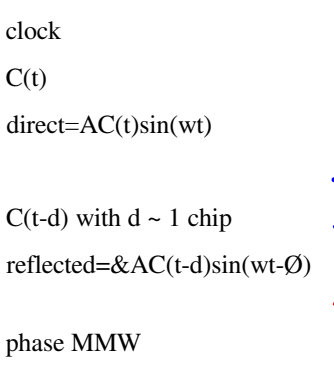

phase MMW

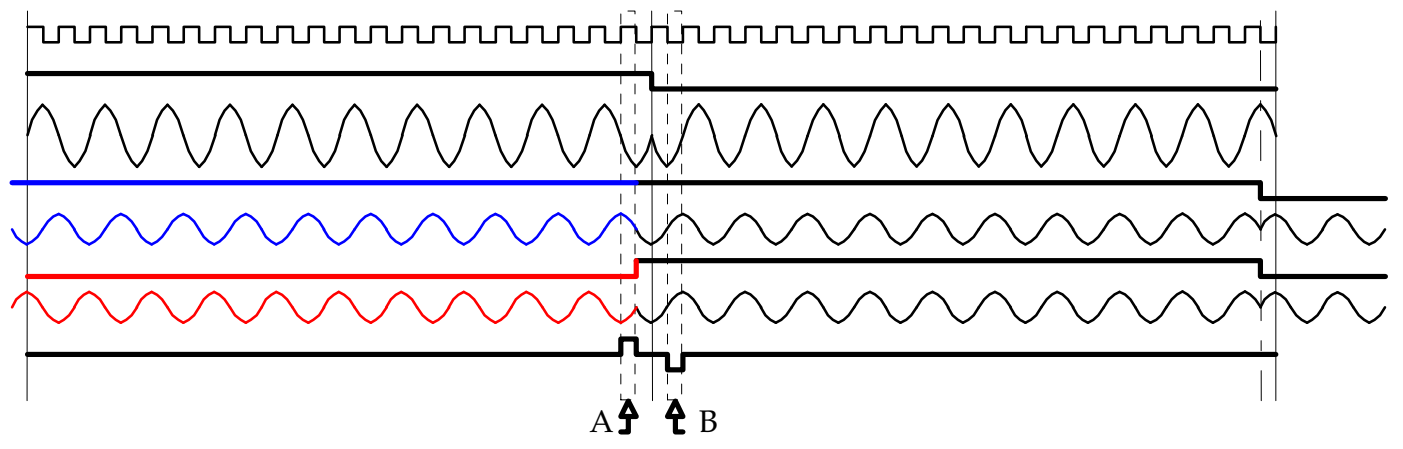

Figure 5.19: one chip delayed multipath signal 
Both cases (same and opposite polarities) have an equal probability of occurrence, since PRN codes have equally distributed transitions and non-transitions. Fig. 5.20 and 5.21 show the phasor diagrams corresponding to both cases. Note that in the case of an opposite polarity of the preceding chip, the vectors corresponding to the two modulations of the MMW do not cancel, but on the contrary these vectors are the same, and cumulate.

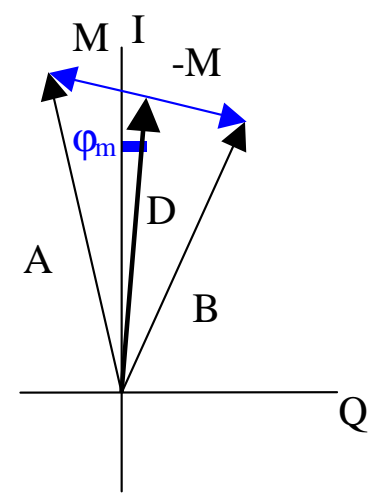

Figure 5.20: phasor diagram of direct and multipath signals corresponding to the MMW phase loop correlator, in case of a preceding chip with the same polarity

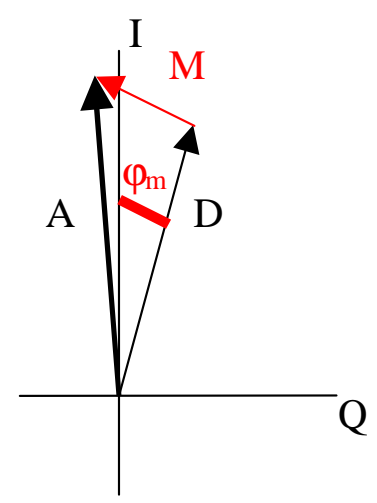

Figure 5.21: phasor diagram of direct and multipath signals corresponding to the MMW phase loop correlator, in case of a preceding chip with the opposite polarity 
So, for one case out of two (see circles in Fig. 5.22), the tracking loop will be entered a biased composite vector, which entails multipath effect on phase MMW measurements for a delay around one chip.

In the light of the different types of code MMW (see $\S 5.1 .2 .3, \S 5.1 .2 .4$ and $\S 5.1 .2 .5$ ), it appears that a solution to this issue around one chip delayed multipath consists in repeating the MMW at every code clock, which, similarly to code MMW, leads to a "type B" phase MMW. To improve again the signal-to-noise ratio (see $\S 5.1 .2 .5$ ), only the second component is used (the first is suppressed), as shown on Fig. 5.22 on a 15 chips long PRN code. This solution, an "asymmetric" phase MMW, keeps the correlator non-sensitive to multipath (provided the delay is enough), and this even if the reflected code is 1 chip delayed (note that 1 chip advanced would not work).

This solution is again based on the fact that transitions and non-transitions are equally distributed in PRN codes (see $\S 5.1 .2 .5$ ).

Symmetric MMW at code transitions
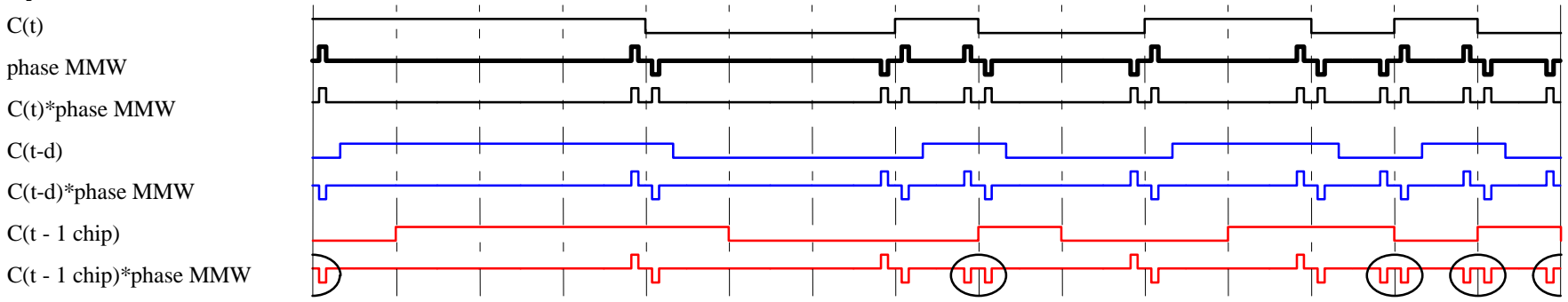

Asymmetric MMW at every code clock
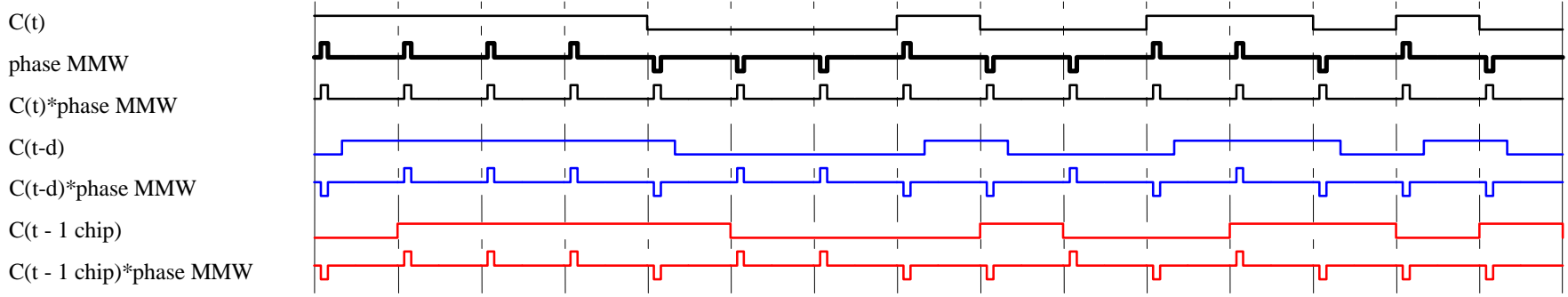

Figure 5.22: MMWs "symmetric at transitions only" and "asymmetric at every code clock" 
Note: a complete set of phase MMWs is given in Appendix 1 ("symmetric at transitions only", "symmetric at every code clock", "asymmetric at transitions only" and "asymmetric at every code clock") with their correlation with advanced/delayed code.

\subsubsection{3 - Signal-to-noise ratio}

Compared to a standard phase loop, the signal content is divided by 40 (1 sample instead of 40 per code chip). As a consequence, the signal-to-noise ratio (SNR) is reduced by $10 * \log _{10}(1 / 40)$ (i.e. $\left.16 \mathrm{~dB}\right)$.

In practice, it is impossible to drive the phase loop with I and Q outputs from the MMW correlator because of its high sensitivity to noise. The solution adopted in Leica's implementation is to keep the phase tracking process unchanged by integrating I and Q in the standard loop at the usual periods of respectively $20 \mathrm{~ms}$ for I (in order to determine the data bit) and $5 \mathrm{~ms}$ for $\mathrm{Q}$ (see Chapter 3, § 3.5). The MMW correlator operates in parallel and outputs $I_{M M W}$ and $Q_{M M W}$ with its original sampling of 1 per 40 clock samples, but with a much larger integration period, in order to improve the SNR. A one second integration period is generally employed. Of course this leads to time correlation in the output values of $\mathrm{I}_{\mathrm{MMW}}$ and $\mathrm{Q}_{\mathrm{MMW}}$, which might be noticeable in high kinematic applications.

Thus, the phase measurements remain biased in the presence of multipath, but the additional observables $I_{M M W}$ and $Q_{M M N}$ output by the MMW correlator enable the correction of this bias. The phase multipath error $\varphi_{\mathrm{m}}$ is simply given by.

$\varphi_{\mathrm{m}}=\arctan \left(\mathrm{Q}_{\mathrm{MMN}} / \mathrm{I}_{\mathrm{MMN}}\right)$

which can be directly applied to the measured phase.

\subsubsection{4 - Phase MMW sampling strategy}

§ 5.1.3.2 already mentioned that the code loop controls the code transitions to occur exactly between the two clocks of the two code MMW samples on the average. 
In the actual Leica phase MMW implementation, the samples that are taken are those following immediately the received code clocks (either transitions or not). So, their distance to the received code transition is comprised between 0 and 1 sample clock. The phase MMW, over time, is the average of the signal that is from 0 to 1 sample clock following the code transitions. So this average is partially contaminated by multipath having an additional path length less than the theoretical $7.5 \mathrm{~m}$ (see $\S 5.2 .4 .1$ ).

\subsection{5 - Conclusions about Phase Multipath Mitigation Window process}

The phase MMW technique enables the measurement of the phase of the direct signal by vector summing (integrating) the composite vector before every code transition with the composite vector immediately after such transitions (but before the arrival of the transitions on any reflected signals). A condition of efficacy that applies to this process is that the additional path length of the reflected signal be at least $7.5 \mathrm{~m}$ in theory, and only half this value in practice, thanks to the optimal choice for the phase MMW digital implementation.

In fact, the phase loop is still driven by a standard correlator, whose multipath error is measured by the MMW correlator provided that the additional path length is enough.

\section{3 - Overall conclusions about MMW}

By using a code multipath mitigation window instead of a classical early minus late, either wide or narrow, the effect of any reflected signal that is delayed beyond half the MMW size is completely eliminated (refer to the original symmetric size, if the MMW is asymmetric).

The efficiency of the receiver to reduce code multipath is almost guaranteed for medium and long reflection delays. However, short delays (below a few tens of metres), caused by reflectors in the nearby environment, still cause multipath error (of a few metres maximum) in code data. 
Thanks to an optimal implementation, the value of the critical delay is in fact around $10 \mathrm{~m}$, with a maximum multipath code error of $2 \mathrm{~m}$.

As far as the phase is concerned, a standard phase loop is still used in Leica receivers but another, in parallel, provides a measurement of the phase multipath error.

Phase multipath error remains for short delays too. The theoretical critical delay is $7.5 \mathrm{~m}$. A practical value to consider is rather half $(3.75 \mathrm{~m})$ thanks again to the implementation. 
Chapter 6

\section{TESTING THE PHASE MULTIPATH MITIGATION WINDOW}

Chapters 6 and 7 focus on the phase MMW process (also called PMMW), and they contain the original contribution of this research. Chapter 6 summarises results of tests performed in both static and kinematic modes (with the SESSYL tests bench in the Laboratoire Central des Ponts et Chaussées, Nantes, France) that permitted an assessment of the performances of the phase MMW process. In order to assess rigorously the effectiveness of this technology, it has been necessary to establish a well-defined testing methodology that is presented first. The methodology that is proposed can be generalised to other phase multipath mitigation techniques [BÉTAILLE, 2003].

A preliminary campaign of tests was done in 2002, and a more complete one in 2003.

\section{1 - The GPS equipment used and the SESSYL facility}

\subsection{2 - The GPS equipment used}

Leica developed and implemented (with C/A-code and L1 carrier) both the code and the phase MMW processes (see Chapter 5) a few years ago, and the ASIC used in the Leica System 500 series already included this technology. For the customer release of this series however, the I and Q provided by the phase MMW correlator were never used in the phase data output, although they were delivered by the ASIC. Only the code data output was effectively upgraded to take advantage of the code MMW process, since its efficacy had already been demonstrated. 
A rigorous assessment of the performances of the phase MMW process was included as part of the research investigations reported in this thesis.

Leica provided a new firmware where the acquisition of $I_{P M M N}$ and $Q_{P M M N}$ is specifically implemented for tests purpose. This chapter addresses the issue of testing the phase MMW correlator and reports on a couple of experiments that took place at the LCPC with two modified System 500 units (SR530) with lightweight antennas (AT502).

Note: in 2002, several preliminary tests were also done with choke-ring antennas (AT504). Investigations mainly concentrated on lightweight antennas in the second campaign of tests in 2003, in order to emphasize the prevalence of the receiver design (i.e. the phase MMW technique) over to the antenna design.

\subsection{2 - SESSYL and the multipath testing equipment}

The SESSYL tests bench belongs to the Site Robotics and Positioning subdivision of the LCPC (Laboratoire Central des Ponts et Chaussées - the French public works research institute). In the frame of its research activity in the domain of site robotics, the LCPC has been equipped since 1995 with a test and research facility devoted to real-time and fullscale positioning systems evaluation. The facility is called SESSYL and is situated in Nantes. Fig. 6.1 shows a picture of SESSYL.

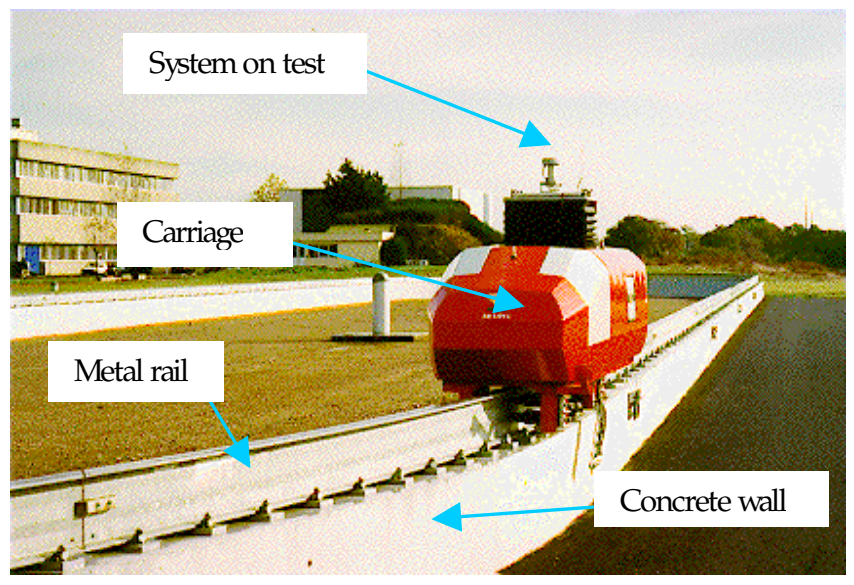

Figure 6.1: SESSYL carriage on its track 
It consists of a closed track (overall dimensions $81 \mathrm{~m} \times 16 \mathrm{~m}$ ), composed of a metal rail fixed upon a concrete wall, with a mobile carriage running on it. The upper part of the carriage is a platform, which can be varied in height, roll and pitch. Thus, any system to be tested is installed on the platform and can describe a reference trajectory in terms of position and attitude angles, accurately known and perfectly repeatable. Two ranges of speed are available: a range corresponding to the speeds of profiling equipment, from 0 to $1 \mathrm{~km} / \mathrm{h}$ and a range corresponding to the speeds of earth-moving and surfacing equipment, from 1 to $15 \mathrm{~km} / \mathrm{h}$. The deviations between the reference trajectory, obtained from internal measurements and static surveys [BÉTAILLE et al., 2000], and the trajectory delivered by the tested system allows the determination of the performances of the system, such as accuracy, but also other interesting features, such as re-initialisation time.

For the purpose of generating multipath in a controlled environment, an experimental set-up additional to SESSYL has been designed that includes a large metallic reflector (a $5 \mathrm{~m}$ by $2.5 \mathrm{~m}$ steel panel, visible in Fig. 6.2). This can be used to generate multipath with different characteristics depending on the distance that it is placed from an antenna.

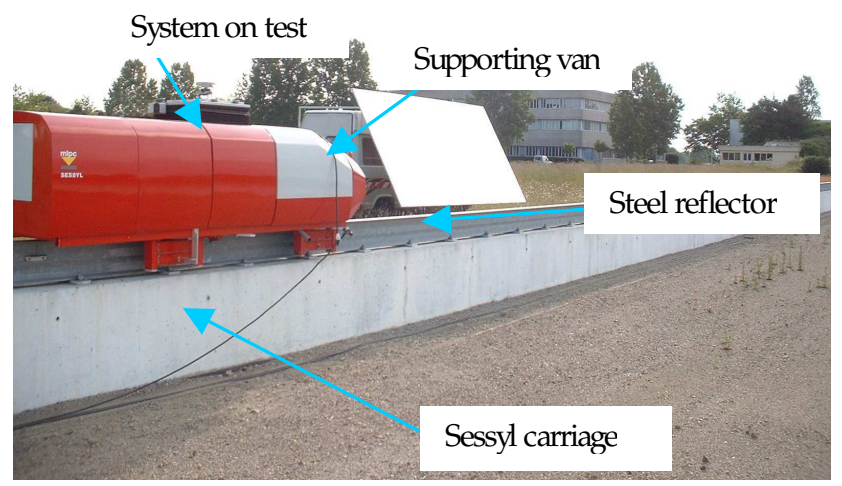

Figure 6.2: SESSYL carriage near the multipath panel

The metallic reflector specially constructed to support the tests was fixed to the side of a van parked in the vicinity of the rover station for static tests or along the SESSYL track for kinematic tests (see Fig. 6.2 and 6.4). The reflector was always placed north of the antenna to face a maximum number of satellites. In practice, its orientation was driven by 
the SESSYL track, which deviates from the geographic North by $12.344^{\circ}$. It was tilted as much as possible from the vertical in order to avoid creation of multipath from low elevation satellites (as actually the signals from these satellites may have already been perturbed by diffraction at the horizon), whilst avoiding the creation of multipath from satellites with an elevation greater than a certain angle (these are used further as differencing satellites in a double differencing process necessary for the multipath mitigation technique assessment). These two specifications are incompatible, and finally the tilt angle $t$ was computed so that the maximum elevation of a satellite affected by multipath is $70^{\circ}$. The geometric formula given in Eq. 6.1 is obtained by applying trigonometric relation in the triangle $\mathrm{OCU}$, where $\mathrm{OC}=\mathrm{d}$ (distance between the antenna and the centre of the panel) and $\mathrm{CU}=1 / 2=1.25 \mathrm{~m}$. Eq. 6.1 considers the 3D problem in the median vertical plane, as shown in Fig. 6.3, which corresponds to the "worst case" (i.e. the case that maximizes the elevation of an affected satellite).

$$
\begin{aligned}
\mathrm{el}=\mathrm{t}+90^{\circ}-\arcsin \left(\mathrm{d} \cdot \cos (\mathrm{t}) / \sqrt{\mathrm{d}^{2}+\mathrm{l}^{2} / 4+\mathrm{d} \cdot \mathrm{l} \cdot \sin (\mathrm{t})}\right)<70^{\circ} \\
\mathrm{l}=1 \mathrm{~m}=\mathrm{t}=13^{\circ} \\
\mathrm{d}=4 \mathrm{~m}=>\mathrm{t}=28^{\circ} \\
\mathrm{d}=7 \mathrm{~m}=>\mathrm{t}=30^{\circ}
\end{aligned}
$$

$2 \mathrm{D}$ profile view panel $2.5 \times 5.0 \mathrm{~m}(1=2.5 \mathrm{~m})$ facing $12.344^{\circ}$ north

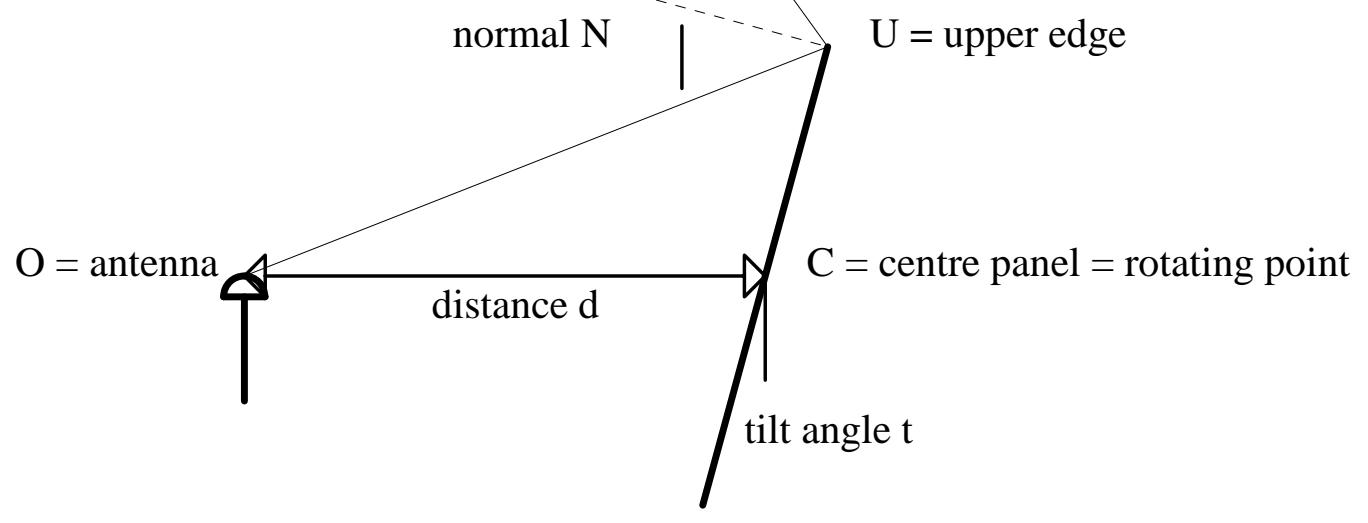

Figure 6.3: set-up of the panel tilt angle. 
Note: the base station was installed in the same grass field where SESSYL is built (see Fig. 6.4), and the baseline length was less than $100 \mathrm{~m}$. Both antennas were oriented parallel. An aerial photo is attached in Appendix 3.

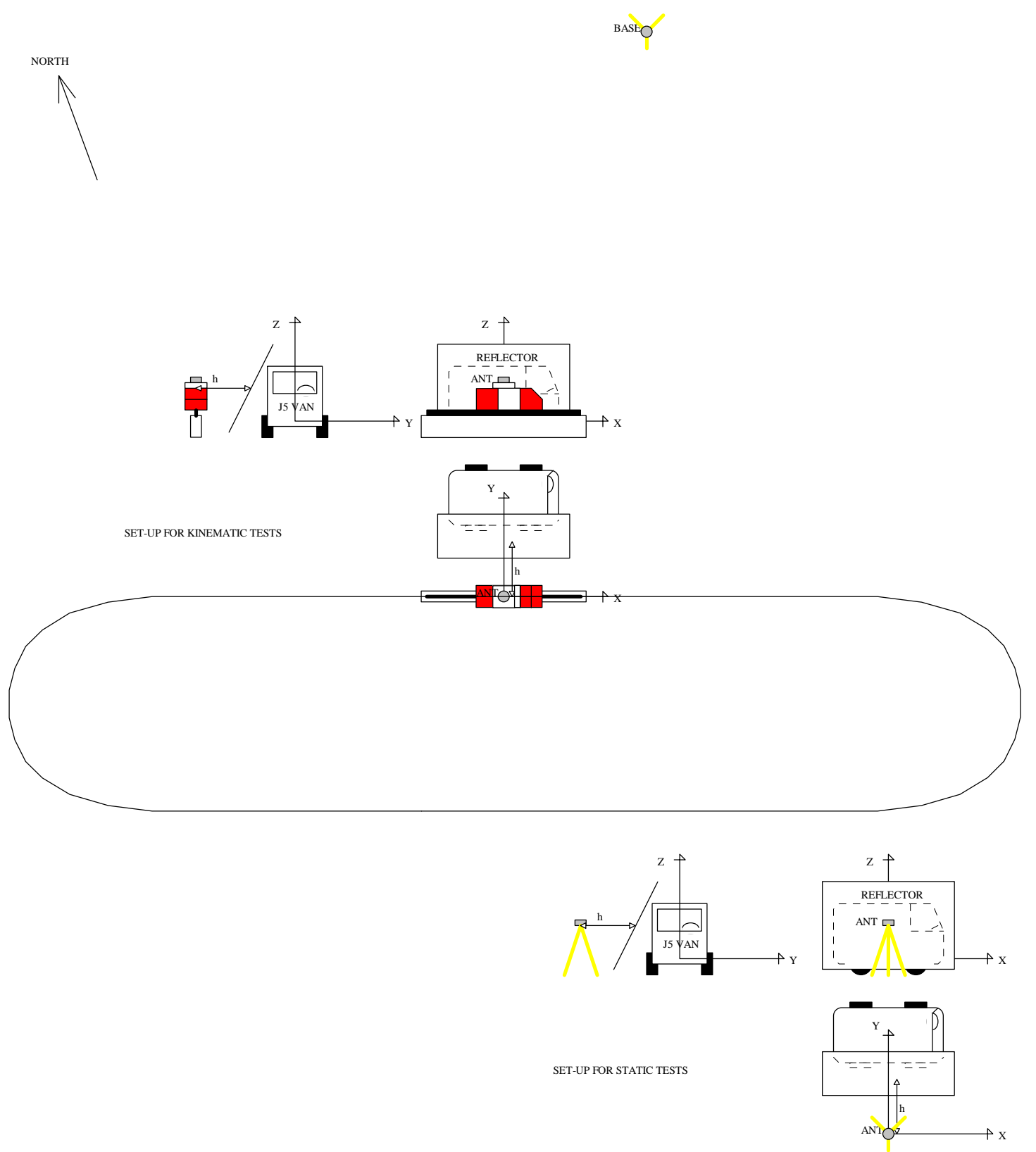

Figure 6.4: static and kinematic tests set-ups 


\section{2 - Prediction of multipath from the panel}

\subsection{1 - Definition of used reference frames}

Different local and global frames are used.

-RL: the SESSYL local reference frame, vertical and orthonormal, determined by four concrete monuments placed on the SESSYL tests site.

The origin of RL is in the vicinity of a hammer mark on the SESSYL track, the $X$-axis is near the first straight section, the $\mathrm{Z}$-axis is vertical and the $\mathrm{Y}$-axis is normal to the $\mathrm{X}$ and Z plane.

Note: the four monuments M1, M2, M3, M4 (Fig. 6.5) have been built with deep foundations and are permanent and fixed.

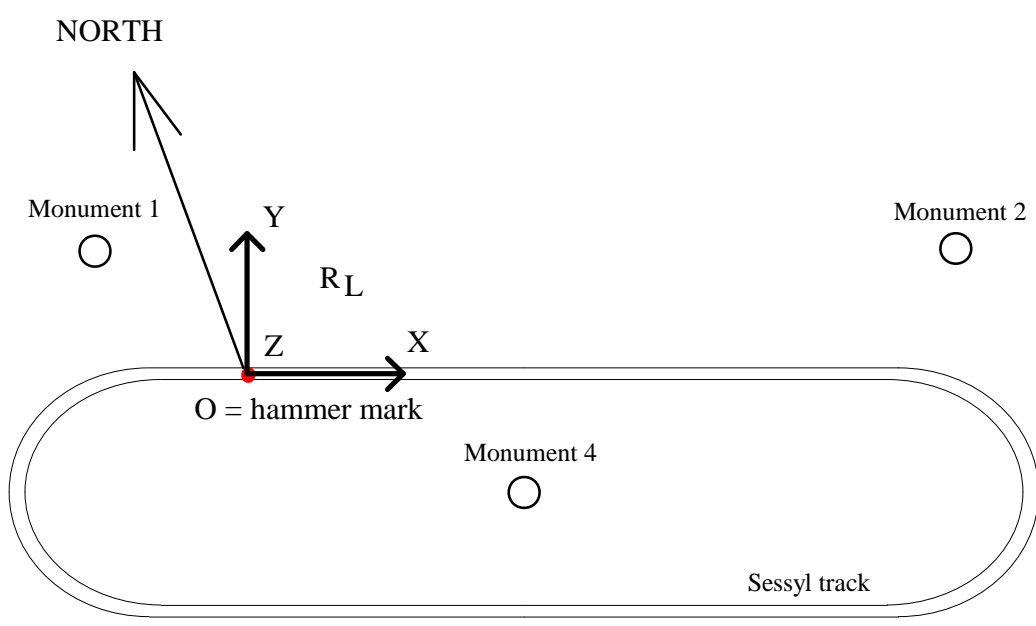

Monument 3

$\bigcirc$

Figure 6.5: the SESSYL local reference frame

- RO: the translation of RL onto the L1 phase centre of the rover antenna (denoted O), near which the reflector is placed. 
- RGF: the geodetic reference system in France.

RGF (Réseau Géodésique Français) is a reference network of the European Terrestrial Reference Frame in France, with an accuracy of the order of a single centimetre. It was set up by the Institut Géographique National (IGN).

The seven-parameter transformation between the SESSYL local reference frame and the RGF was established soon after the construction of SESSYL, in a geodetic campaign during which the monuments were surveyed by GPS along with several local RGF points.

Hence, the GPS co-ordinates given by a GPS unit that is being tested on SESSYL can be converted into RL in order to be compared to the reference SESSYL trajectory. Or inversely, points in this trajectory can be converted into RGF to compute, for example, observed-computed double differences of phase (O-C DD). This is what has been done in all of the tests reported in this chapter. Note that the seven-parameter geodetic transformation is valid only for points situated in the SESSYL area.

\subsection{2 - Computation of the multipath window time zones}

This section describes the computation of the multipath window time zones that the different satellites enter during the tests. These zones can be computed before the experiment (as far as an approximated position of the panel is given). Afterward, the precise position is surveyed with a total station during the tests, which enables the multipath window time zones to be confirmed and statistics to be computed for these zones only.

Firstly, let us suppose a two-dimensional scheme. Fig. 6.6 displays an example (solid line) of direct and reflected paths, as well as the two limits (dotted lines) corresponding to direct and reflected paths near the edge of the reflector. By applying the basic law of geometric optics, one can state that any source (i.e. any satellite in the three-dimensional scheme) situated in the area determined by these limits and the reflector itself will be received both directly and after a reflection. 


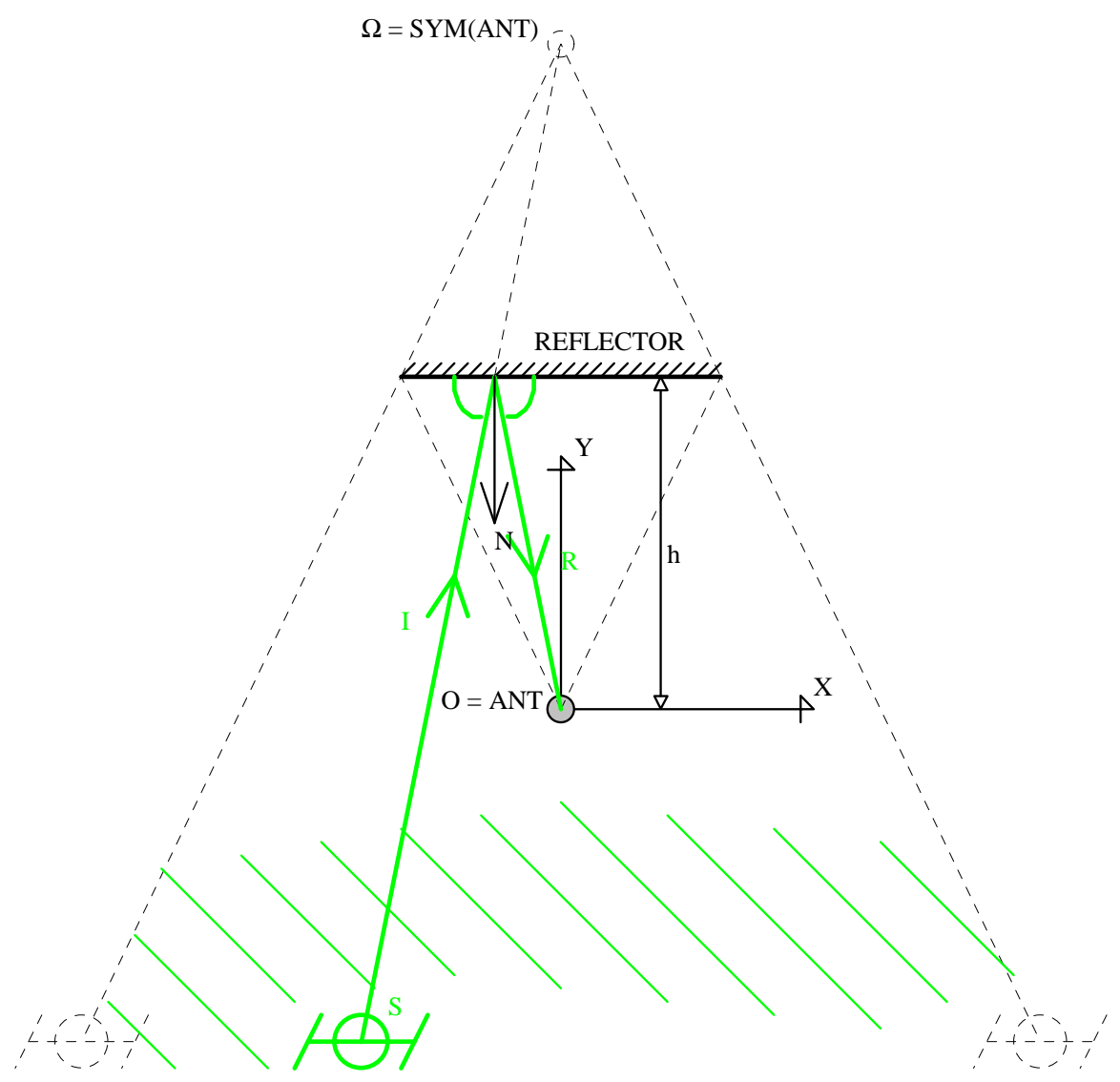

Figure 6.6: the reflection in a 2-dimensional scheme

Secondly, the computation is extended to a three-dimensional scheme.

To compute the positions of the satellites in $\mathrm{RO}$ (the local reference frame centred at the L1 phase centre of the rover antenna), the seven-parameter geodetic transformation obviously cannot be used (it is valid only locally). The approximate position of a satellite (denoted S) in RO can be derived from its elevation and azimuth, denoted respectively "el" and "az". These angles are themselves derived from the position of the satellite obtained from the broadcast ephemeris and raw binary data logged, these being converted into the RINEX format and processed in a single epoch code based GPS solution developed at the LCPC under Matlab [DURAND, 2003]. This computation is very classical and many other software packages exist that can deliver similar results. 
So, given el and az, the co-ordinates of satellite $\mathrm{S}$ in $\mathrm{RO}$ is:

$$
\begin{array}{ll} 
& \left\{\mathrm{R} \cos \left(90^{\circ}+\mathrm{az} 0-\mathrm{az}\right) \cos (\mathrm{el})\right. \\
& \left\{\mathrm{R} \sin \left(90^{\circ}+\mathrm{az} 0-\mathrm{az}\right) \cos (\mathrm{el})\right. \\
& (\mathrm{R} \sin (\mathrm{el})
\end{array}
$$

where $\mathrm{R}$ is the geometric distance from the L1 phase centre of the rover antenna to the satellite $S$, and $\mathrm{azO}$ is the azimuth of the local reference frame compared to the geographic North (around $12.344^{\circ}$, with an error less than $1 \mathrm{~cm} / 100 \mathrm{~m}$, i.e. $0.005^{\circ}$ ). This level of error was estimated on the basis of a GPS static survey of the SESSYL monuments [BÉTAILLE et al., 2000]).

A total station, set-up in the local reference frame RL, was used to determine the position of the reflector with an accuracy of $1 \mathrm{~mm}(1 \sigma)$. The accuracy of the co-ordinates of the panel is directly linked to the adaptation of the instrument into RL, which is done by surveying 3 of the 4 local monuments, and also the skill of the operator. Twelve optical targets (Fig. 6.7) were stuck to the surface of the panel (ten at the edges and two at the centre), and surveyed by the total station. Afterwards the best plane was computed, in the least squares sense, and all the twelve surveyed points projected onto this plane. The plane was then delimited in space by the intersection of the surveyed edges (i.e. the corners), these being offset by a few centimetres corresponding to the distance between the optical targets (see Fig. 6.8) and the real edge of the structure.

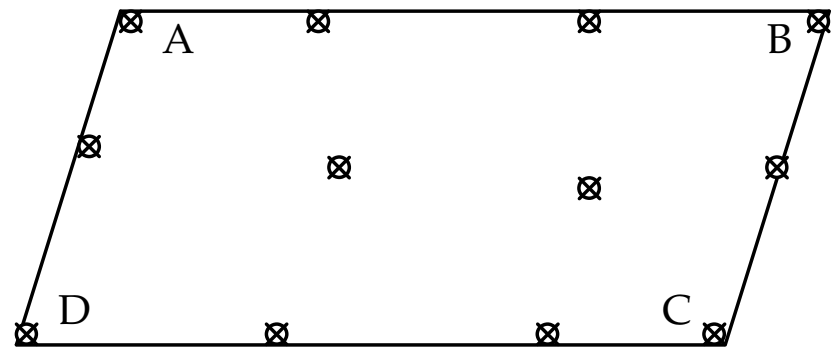

Figure 6.7: the 12 optical targets stick on the panel

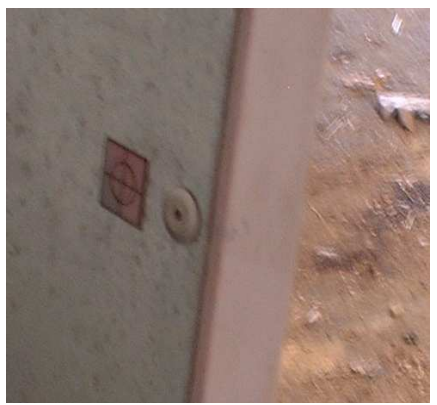

Figure 6.8: an optical target 
The co-ordinates of the rover can be determined by GPS from one of the local monuments by a static survey of a few hours, or directly with the total station, like for the reflector, the antenna being removed and replaced by a target. The estimated error of the survey of the rover antenna is less than $5 \mathrm{~mm}$.

Finally, in the following computation, the position of the reflector is determined by the co-ordinates of its four corners (denoted A, B, C and D) in the local frame.

The co-ordinates of the image point (denoted $\Omega$ ) of the L1 phase centre of the rover antenna are computed.

$\Omega=\operatorname{sym}_{/ \mathrm{ABCD}}(\mathrm{O})$

The intersection (denoted I) of the line between $\Omega S$ and the plane $\mathrm{ABCD}$ is computed. If $\mathrm{I}$ is contained in the rectangle $\mathrm{ABCD}$ delimiting the panel, then there is a specular reflection. The corresponding is represented with "green stripes" in Fig. 6.5.

The track of the point I on the plane $\mathrm{ABCD}$ can be represented graphically at any time. When it reaches the edge of the panel, the specular reflection stops and becomes diffuse. At this time, diffraction occurs, and part of the signal (in terms of energy) that was reflected before is diffused then, which entails less interference with the direct signal, and less multipath errors in the measurements. Then, errors are back to their nominal level (i.e. a level when no specific and strong multipath is created, which corresponds to ambient and relatively weak multipath always present in the environment).

Another situation of diffraction needs to be considered in the context of the experiment: this relates to satellites that are hidden behind the panel but still visible in terms of signal strength. A geometrical computation, similar as that for multipath, enables the zones when such diffraction occurs to be defined (Fig. 6.9). Diffraction zones are represented with "red stripes" in this figure. Note also that diffraction at the horizon always exist when the elevation is under $15^{\circ}$. Its effect, as well as that of diffraction due to the panel, causes the errors in measurements to increase unboundedly. 


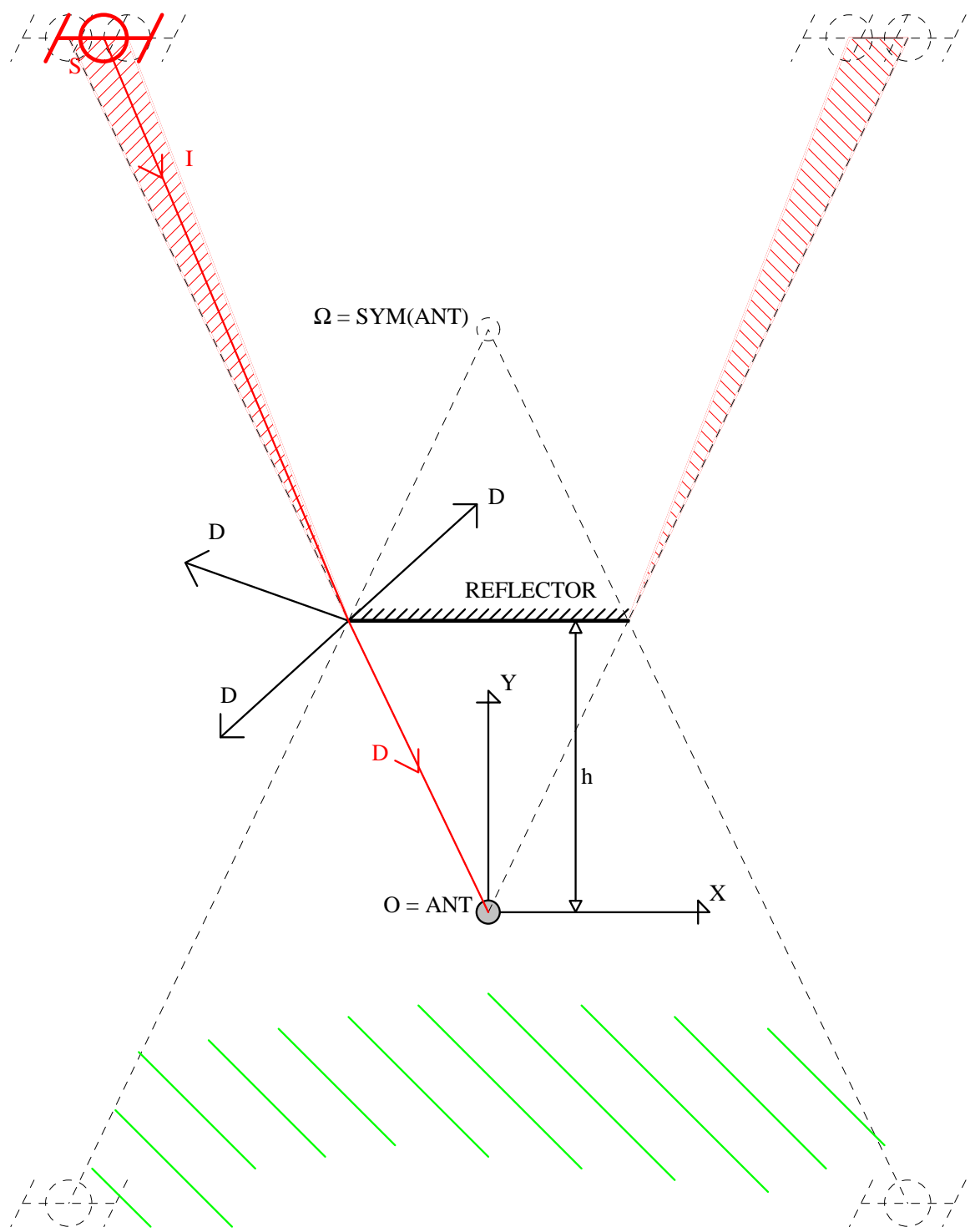

Figure 6.9: the diffraction in a 2-dimensional scheme

Another interesting representation of the multipath zones is given in Fig. 6.10, which corresponds to the campaign of tests carried out in 2003 with distances of 1,4 and $7 \mathrm{~m}$ between the antenna and the panel. These zones are displayed in an azimuth and elevation polar representation, like in a sky plot.

Note: an error was made in the Matlab programming of Eq. 6.1 (" $\cos ^{\prime \prime}$ was written instead of "sin"!), and the tilt angles were set-up at $6^{\circ}, 24^{\circ}$ and $28^{\circ}$ (instead of $13^{\circ}, 28^{\circ}$ and $30^{\circ}$ ), which gives maximum elevations of $60^{\circ}, 62^{\circ}$ and $64^{\circ}$ (and not $70^{\circ}$ as initially required). This is visible in Fig. 6.10. There is no significant impact of this error on the validity of the experiment and on the results. 


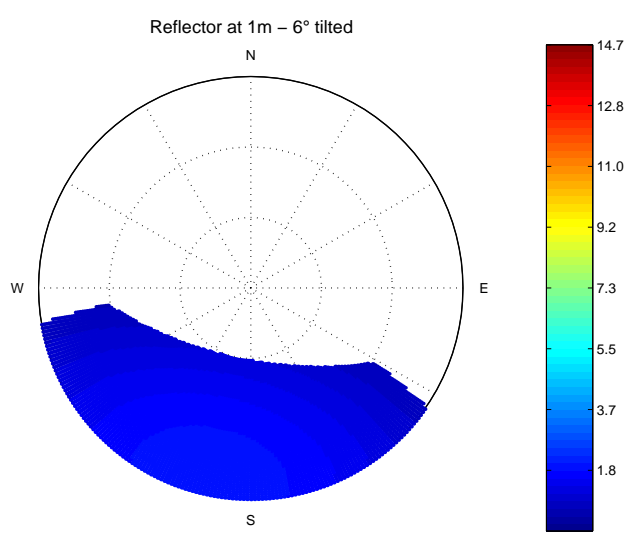

$0.7 \mathrm{~m}<$ addpath $<2.0 \mathrm{~m}$
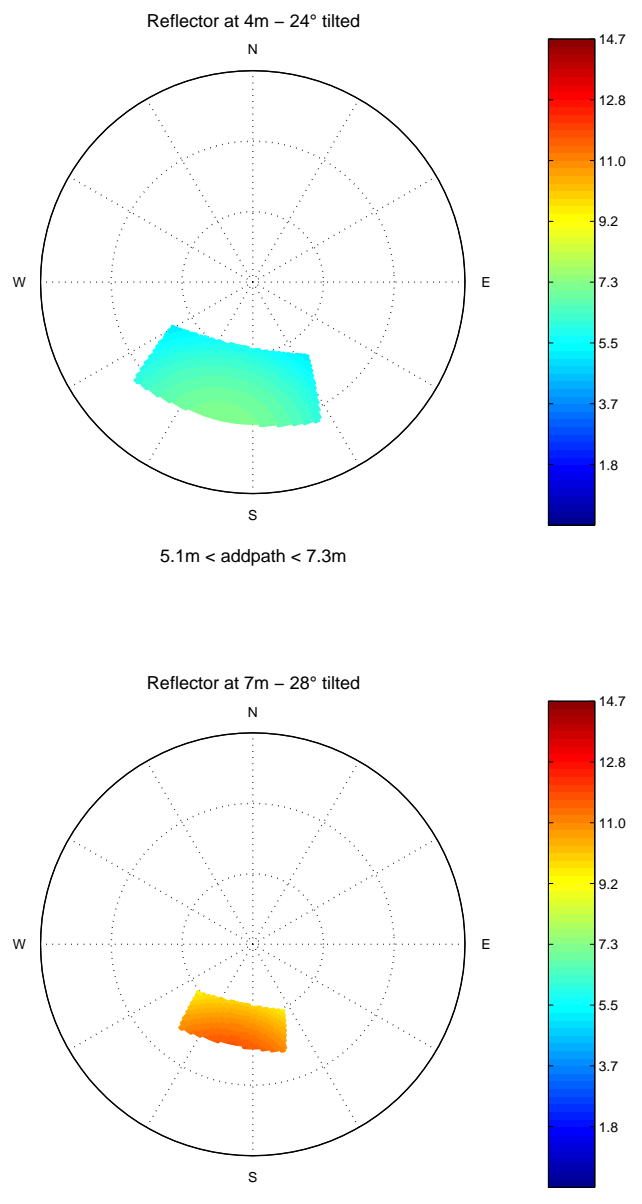

$9.5 \mathrm{~m}<$ addpath $<11.7 \mathrm{~m}$

Figure 6.10: sky plot display of multipath zones, when the panel is placed 1, 4 and $7 \mathrm{~m}$ (the colour bar corresponds to the additional path length travelled by the reflected signal, in $\mathrm{m}$ ). 
One can notice that when the reflector is close to the antenna, it is actually impossible to tilt the panel in order to eliminate both low elevation satellites and differencing satellites from the multipath zone. In this case, the priority has been given to the differencing ones, which entails possible mixed influence of multipath and diffraction in measurements corresponding to low elevation satellites in view.

In Fig. 6.10, the colour bar corresponds to the additional path length travelled by the reflected wave, denoted $\mathrm{L}$. The lower and upper limits of $\mathrm{L}$ are written under the figures. $\mathrm{L}$ (as well as other characteristics of the reflected ray like its elevation and azimuth) can be easily deduced from the geometrical modelling presented before: this is done in $\S$ 6.2.3.

The survey performed with the total station enabled the theoretical multipath window time zones to be computed again according to the actual set-up, close to that designed.

\subsection{3 - Prediction of the multipath phase error}

The direct and reflected signals have been characterised in Chapter 1, and their combination computed in Chapter 4:

$\varphi=\arctan \left(\left[\Sigma_{\mathrm{i}} \alpha_{\mathrm{i}}^{\prime *} \sin \left(\Theta_{\mathrm{i}}\right)\right] /\left[1+\Sigma_{\mathrm{i}} \alpha_{\mathrm{i}}^{\prime *} \cos \left(\Theta_{\mathrm{i}}\right)\right]\right)$

The panel placed near the antenna is considered as the only source of multipath. So $\mathrm{i}=1$ and the multipath phase error $\varphi$ is given by the following formula:

$\varphi=\arctan \left(\sin (\Theta) /\left[1 / \alpha^{\prime}+\cos (\Theta)\right]\right)$

$\Theta$ results from the additional path length $\mathrm{L}$ travelled by the reflected wave, plus the reflection phase shift $\left(180^{\circ}\right)$, plus the antenna phase shift (see Chapter 1$)$. When the source of the signal is a satellite, the distance from the ground antenna to the satellite can be considered as infinite with respect to the distance from the antenna to the reflector. Consequently, all the incident waves in the near environment of the antenna are considered parallel (OS// $\Omega S$ in Fig. 6.6). 
It can be geometrically shown that:

$\Theta=2 \pi \mathrm{L} / \lambda=(4 \pi \mathrm{h} / \lambda) \sin (\theta)$

where

$\mathrm{L}$ is the additional path length $(\mathrm{L}=2 \mathrm{~h} \sin (\theta))$

$\mathrm{h}$ is the normal distance from the antenna phase centre to the reflective surface

$\theta$ is the angle of elevation of the satellite with respect to the surface, or grazing angle, and $\lambda$ is the wavelength.

$h$ results from the computation of the normal distance from the point $\mathrm{O}$ (see Fig. 6.6) to the panel. $\theta$ can be deduced from the scalar product between the normal vector $\mathrm{N}$ (given by surveying the panel) and the unit vector $U$ of the line of sight $O S$.

$\mathrm{U}=\mathrm{OS} /|| \mathrm{OS}||$

$\left\lceil\mathrm{Ux}=\cos \left(90^{\circ}+\mathrm{az} 0-\mathrm{az}\right) \cos (\mathrm{el})\right.$

$\mathrm{U}: \quad\left\{\mathrm{Uy}=\sin \left(90^{\circ}+\mathrm{az} 0-\mathrm{az}\right) \cos (\mathrm{el})\right.$

( $\mathrm{Uz}=\sin (\mathrm{el})$

$\int \mathrm{Nx}$

$\mathrm{N}: \quad\{\mathrm{Ny}$

l $\mathrm{Nz}$

$\theta=90^{\circ}-\arccos ($ N.U $)=\arcsin ($ N.U $)$

$\sin (\theta)=$ N.U

To obtain a complete prediction of the multipath phase error with Eq. 6.5, the modified ratio of amplitude $\alpha^{\prime}$ still remains unknown a priori. An approximation can be made with $\alpha^{\prime}$ : 
$\alpha^{\prime}=\alpha^{*} \mathrm{R}(\tau+\mathrm{d}) / \mathrm{R}(\tau) \sim \alpha^{*}(1-\mathrm{d} / \mathrm{T}) \sim \alpha$

because the multipath code error $\tau$ is considered to be small (see Chapter 4, Eq. 4.12) and the code delay $\mathrm{d}$ is small with respect to the duration of the chip (the panel is placed in the vicinity of the antenna, causing an additional path length of a few metres maximum).

So, the ratio of amplitude only depends on the material and the antenna. In the presence of multipath from a metallic material and under the condition that the grazing angle is above the Brewster angle, The RHCP incident wave becomes LHCP after a reflection. So the computation of $\alpha$ requires the knowledge of both the antenna gain pattern in Left Hand Circular Polarisation (for the attenuation of the reflected signal) and in Right Hand Circular Polarisation (for the amplification of the direct signal).

The ideal solution is to obtain the antenna gain patters in both RHCP and LHCP from a dedicated calibration experiment in an anechoic chamber. Failing that, it is suggested that $\alpha$ is estimated by fitting the amplitude of the predicted multipath phase error with that observed in reality.

Note also that, because it has not yet been calibrated by the manufacturers, the antenna phase pattern (contrary to the antenna gain pattern) is never available to compute the unknown phase shift of the reflected signal $\Theta$. Like the gain, the phase varies with the azimuth and the elevation of the wave relatively to the plane of the antenna.

Finally, to obtain a complete prediction of the multipath phase error with Eq. 6.5, the parameters $\alpha$ and $\Theta$ need to be estimated.

\subsection{4 - Tuning the parameters $\alpha$ and $\Theta$ of the model}

This section presents the tuning of $\alpha$ and $\Theta$ on two data sets (corresponding to SV1 and SV2), collected during the two campaigns of static tests. Table 6.1 shows the planning of the static tests. 


\begin{tabular}{lcccc}
\hline Campaign in 2002 & day147 & day148 & \\
Reflector & no & at $5 \mathrm{~m}, 30^{\circ}$ tilt & & \\
\hline Campaign in 2003 & day209 & day210 & day 211 & day 212 \\
Reflector & no & at $1 \mathrm{~m}, 6^{\circ}$ tilt & at $4 \mathrm{~m}, 24^{\circ}$ tilt & at $7 \mathrm{~m}, 28^{\circ}$ tilt \\
\hline
\end{tabular}

Table 6.1: static tests planning and conditions of tests

As already mentioned in $\S 6.1 .2$, a pair of SR530 receivers and AT502 antennas was used for both campaigns. Note that from the first campaign to the second, the equipment was not the same serial numbers. This last point is particularly important as far as the gain and phase patterns of the antennas are concerned, those being not strictly repeatable with lightweight antennas. The impact on the modelling of $\alpha$ and $\Theta$ is summarised in table 6.2.

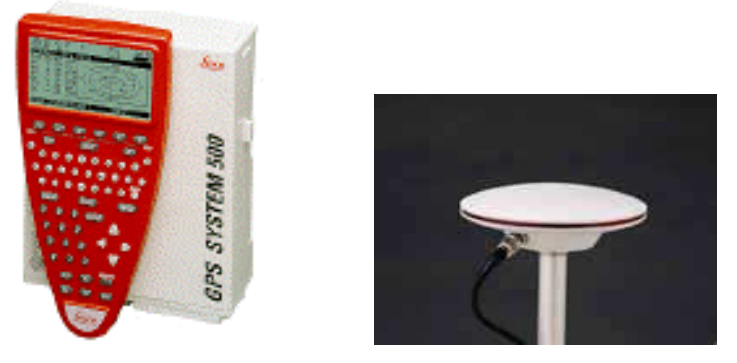

Figure 6.11: SR530 receiver and AT502 antenna

Both base and rover antennas were set on tripods in the grass field surrounding the SESSYL test bed, and oriented parallel. The baseline length was less than $100 \mathrm{~m}$. The $5 \mathrm{mx}$ $2.5 \mathrm{~m}$ metallic reflector was placed near the rover station. During the preliminary static test, the tilt angle was set approximately near $30^{\circ}$. For the tests done during the second campaign, the tilt angle was computed with Eq. 6.1.

The individual multipath phase error, i.e. in a given GPS unit and for a given satellite, is impossible to obtain independently of the receiver and satellite clock offsets. Therefore double differences (DD) of L1 phase, which combined four multipath phase errors, have 
been computed. Also, for short baseline kinematic GPS, the multipath source of error predominates in the DD of phase data. The error in the double differences of phase data is an addition/subtraction of the phase errors corresponding to a pair of satellites and a pair of GPS units. Here, the hypothesis is made that there is no significant multipath at the base station. This has been audited on a specific day for each campaign, without the panel. The conclusion is that the most important multipath is actually due to the metallic reflector.

As a practical consequence, the multipath window time zones for both satellites in the DD must be computed. When these zones intersect, the phase error predicted for the differencing satellite must be subtracted from that predicted for the other satellite. More commonly, when only one of the satellites is affected, its phase error is the only one that contributes to the error in the double differences.

Fig. 6.12a to 6.12c correspond to the preliminary test, and respectively display in two columns (for the two considered satellites):

- the standard deviation between the O-C DD of L1 phase and that predicted by the model, computed within the multipath window time zone (delimited by the "green window'), versus $\alpha$ and $\Theta$;

- the same standard deviation, versus $\alpha$, once $\Theta$ is fixed to its optimum;

- the resulting modelled multipath phase error (the "red line"), superimposed to the time series of the O-C DD of L1 phase: it was computed assuming an infinite panel, which is why it extends beyond the actual multipath window time zone.

The equivalent figures for the tests at 1,4 and $7 \mathrm{~m}$ in the second campaign are given in Fig. 6.13 to 6.14 .

In this tuning, the parameters are supposed to keep constant. Values of $\alpha$ from 0.01 to 0.35 in steps of 0.01 , and values of $\Theta$ from $0^{\circ}$ to $330^{\circ}$ in steps of $30^{\circ}$ have been tried. It is not sensible to divide further since the accuracy of the relative positioning of the antenna and the reflector is a few $\mathrm{mm}$ for a wavelength of $19 \mathrm{~mm}$. 

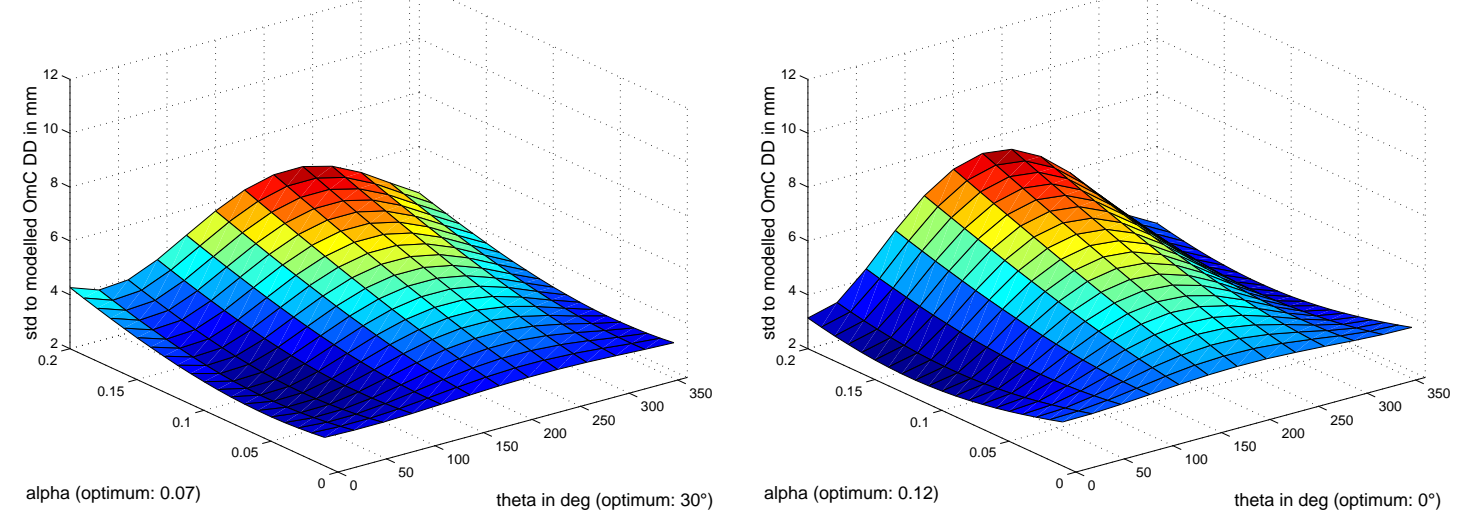

Figure 6.12a: day148 - standard deviation between the O-C DD and the model, versus $\alpha$ and $\Theta$.
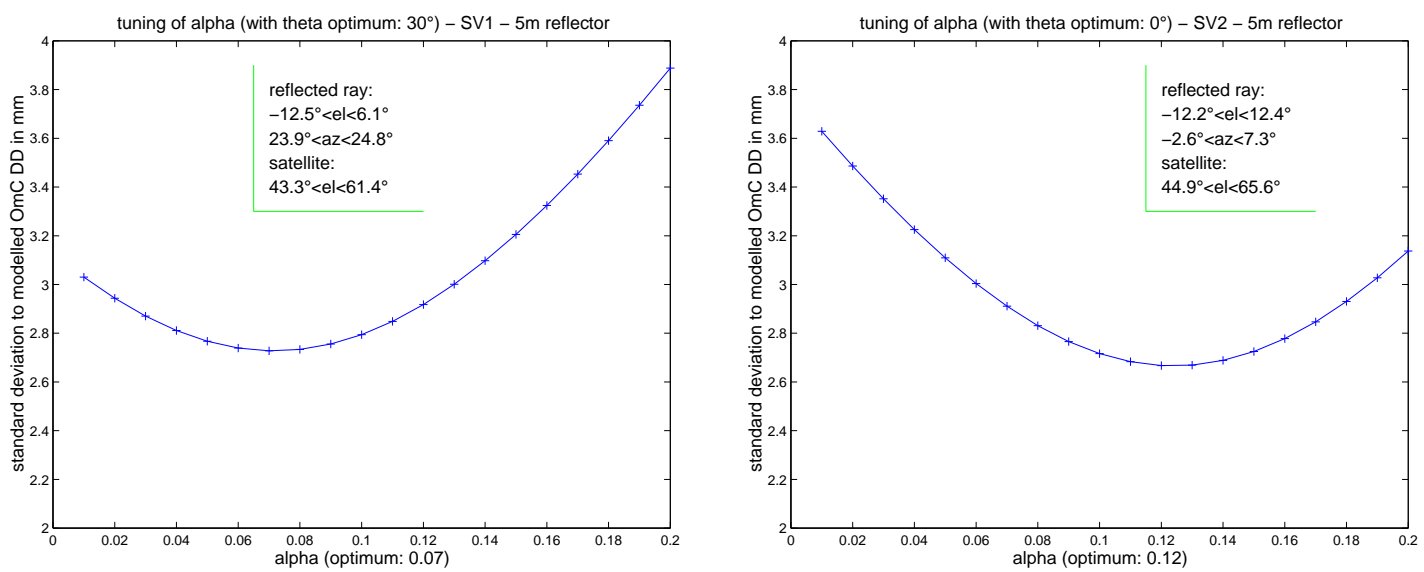

Figure 6.12b: standard deviation between the O-C DD and the model, versus $\alpha, \Theta$ fixed.
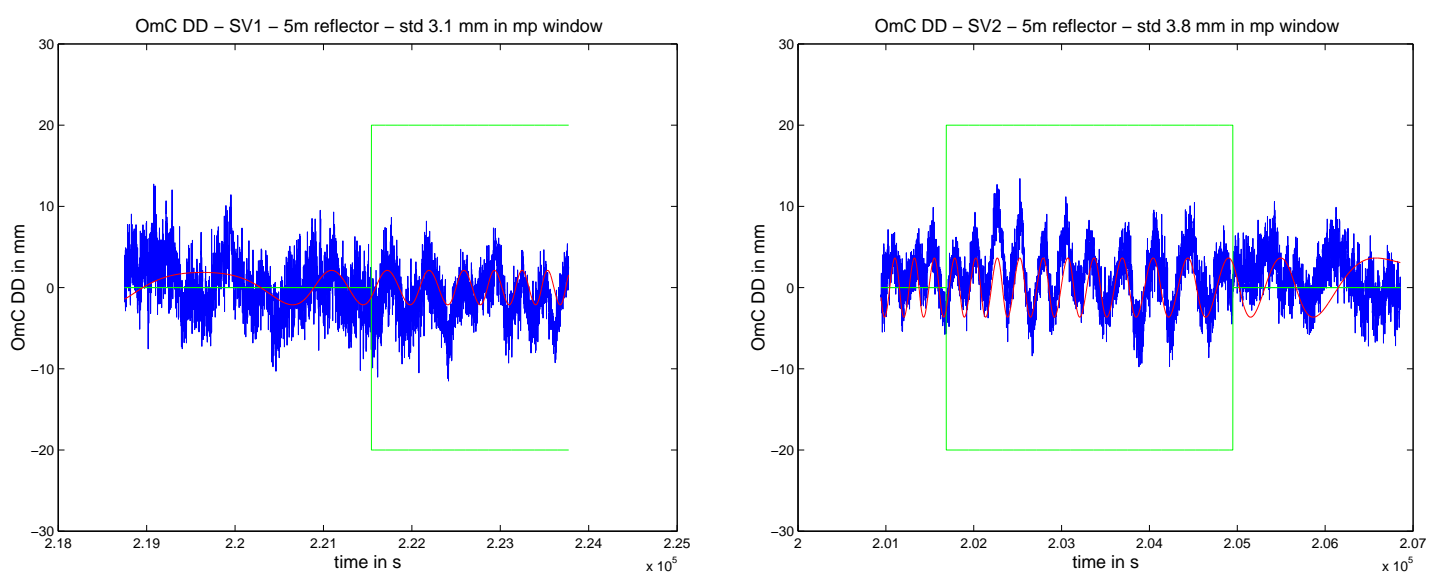

Figure 6.12c: modelled multipath phase error superimposed to the O-C DD. 

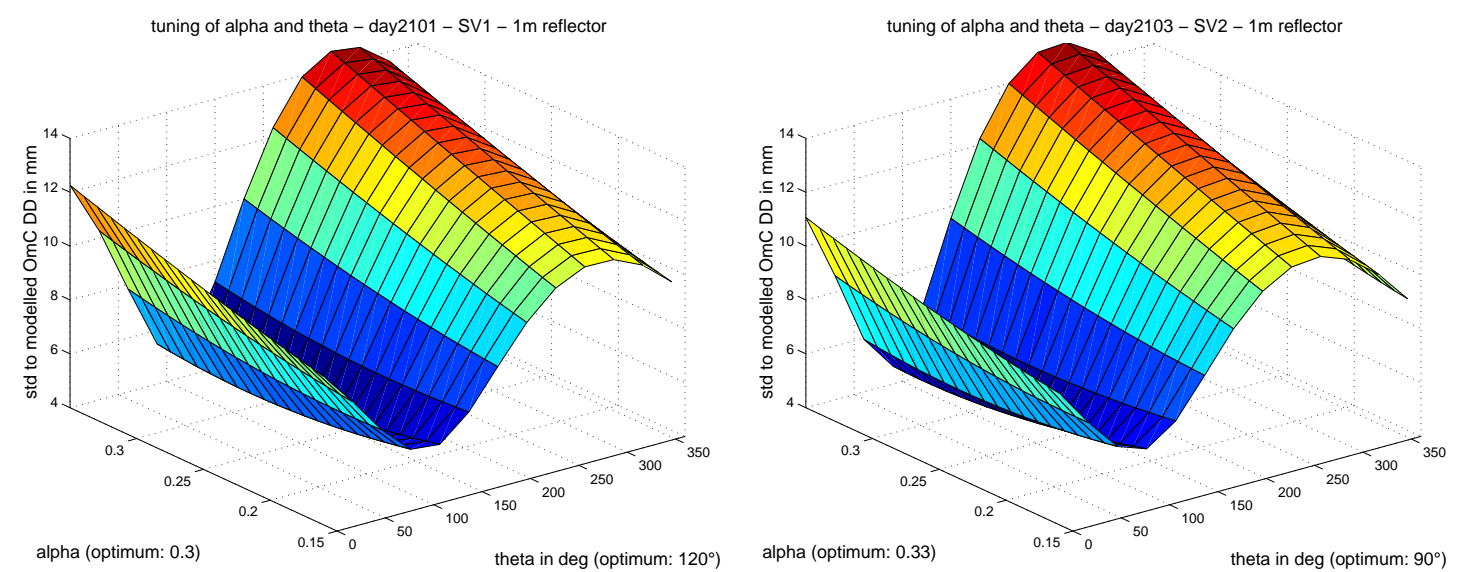

Figure 6.13a: day210 - standard deviation between the O-C DD and the model, versus $\alpha$ and $\Theta$.
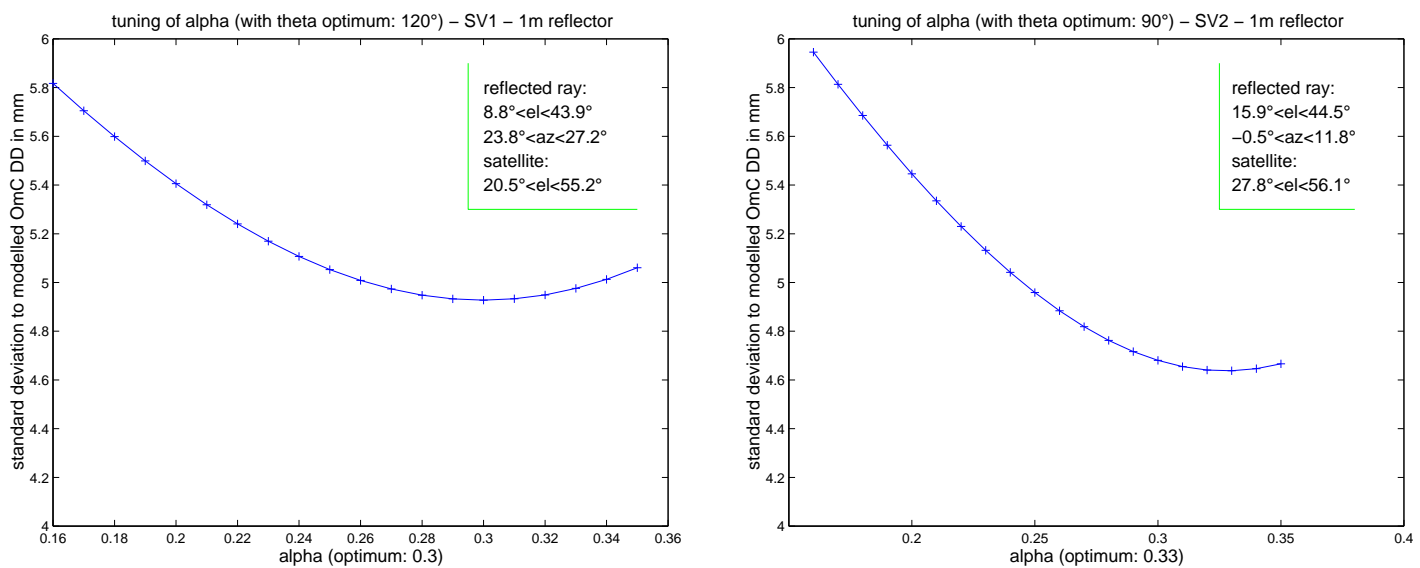

Figure 6.13b: standard deviation between the O-C DD and the model, versus $\alpha, \Theta$ fixed.
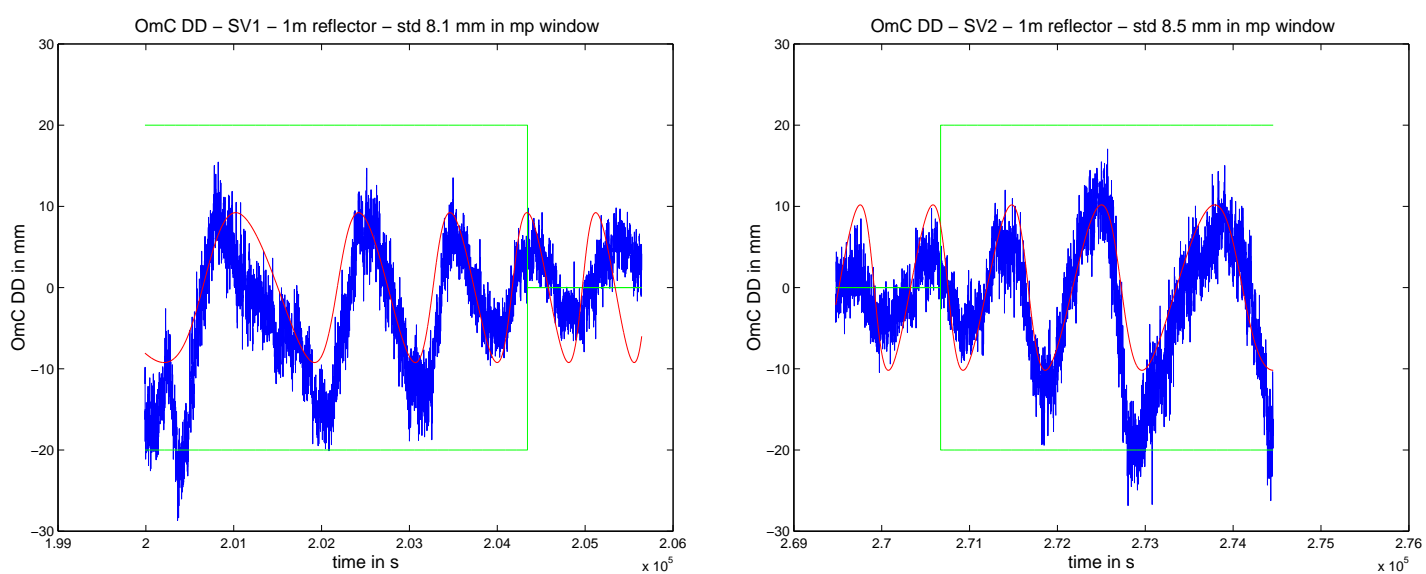

Figure 6.13c: modelled multipath phase error superimposed to the O-C DD. 

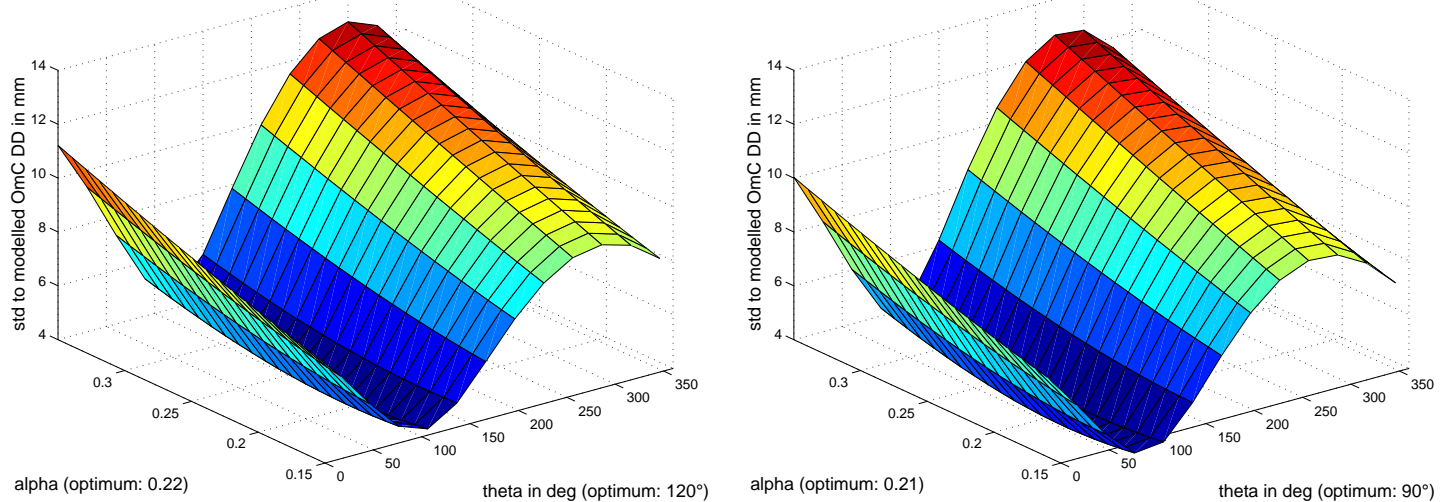

Figure 6.14a: day211 - standard deviation between the O-C DD and the model, versus $\alpha$ and $\Theta$.
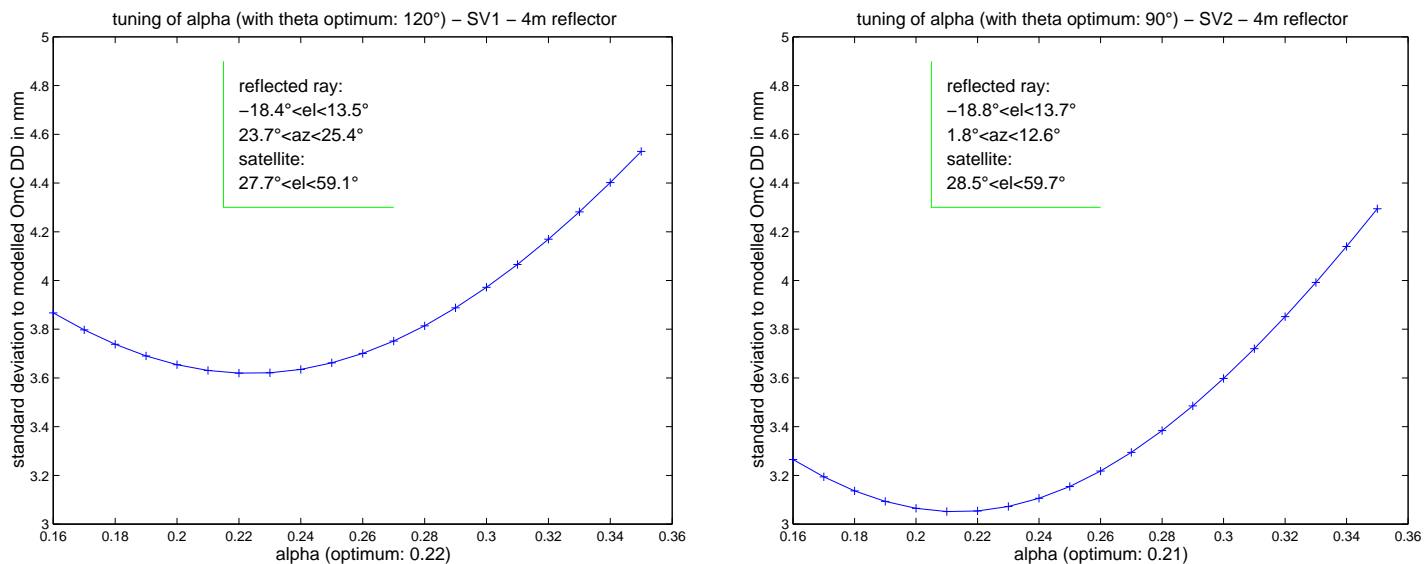

Figure 6.14b: standard deviation between the O-C DD and the model, versus $\alpha, \Theta$ fixed.
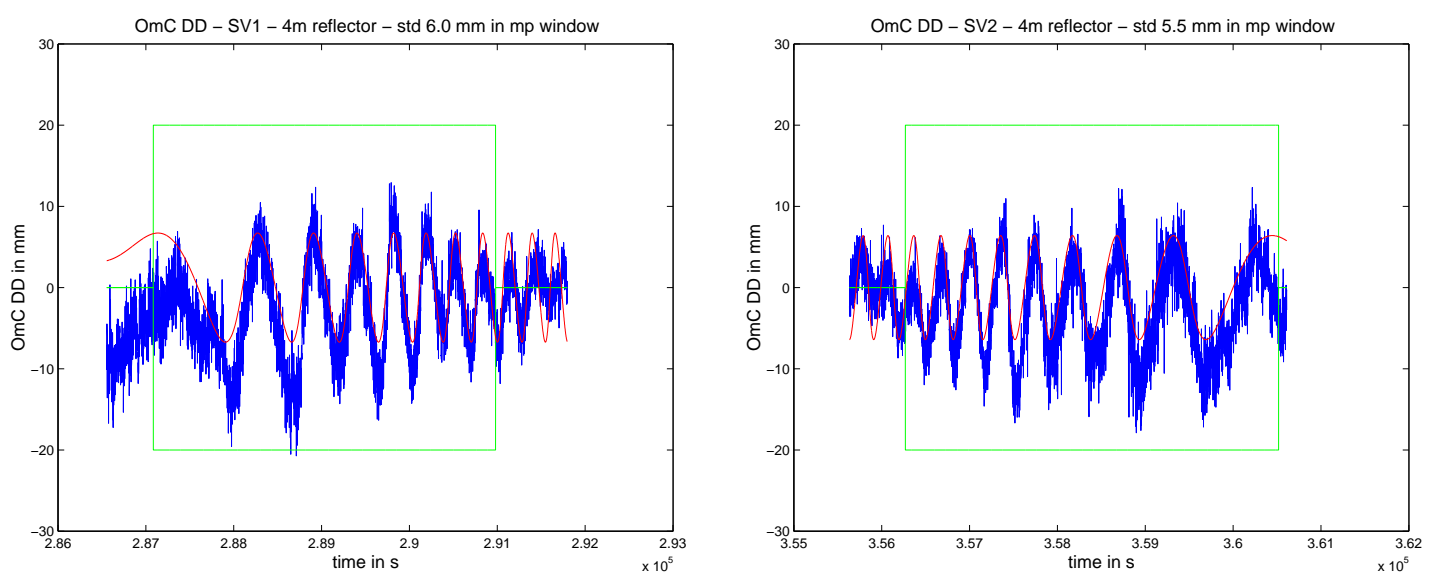

Figure 6.14c: modelled multipath phase error superimposed to the O-C DD. 

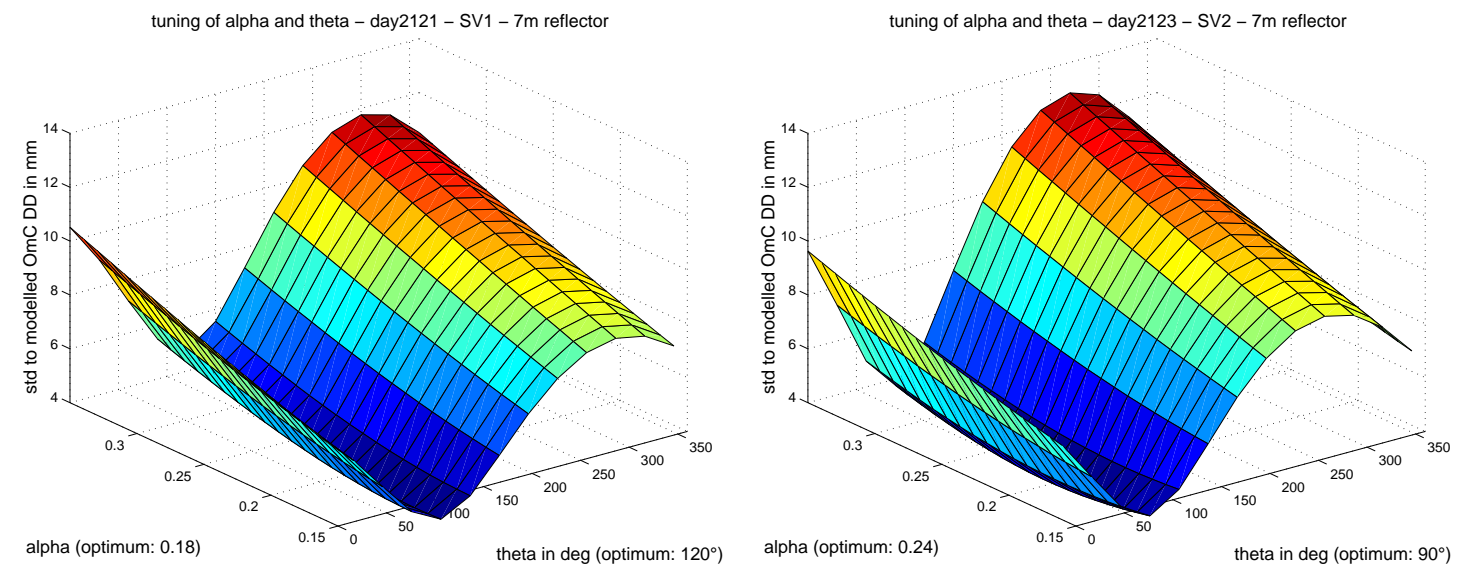

Figure 6.15a: day212 - standard deviation between the O-C DD and the model, versus $\alpha$ and $\Theta$.
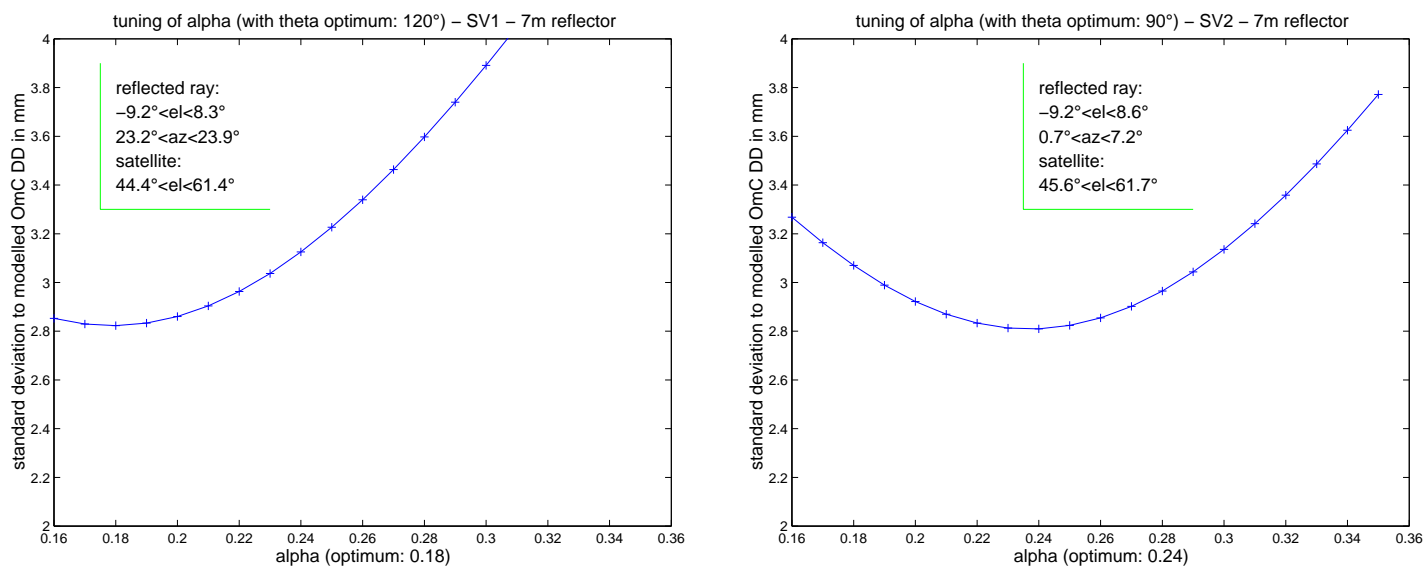

Figure 6.15b: standard deviation between the O-C DD and the model, versus $\alpha, \Theta$ fixed.
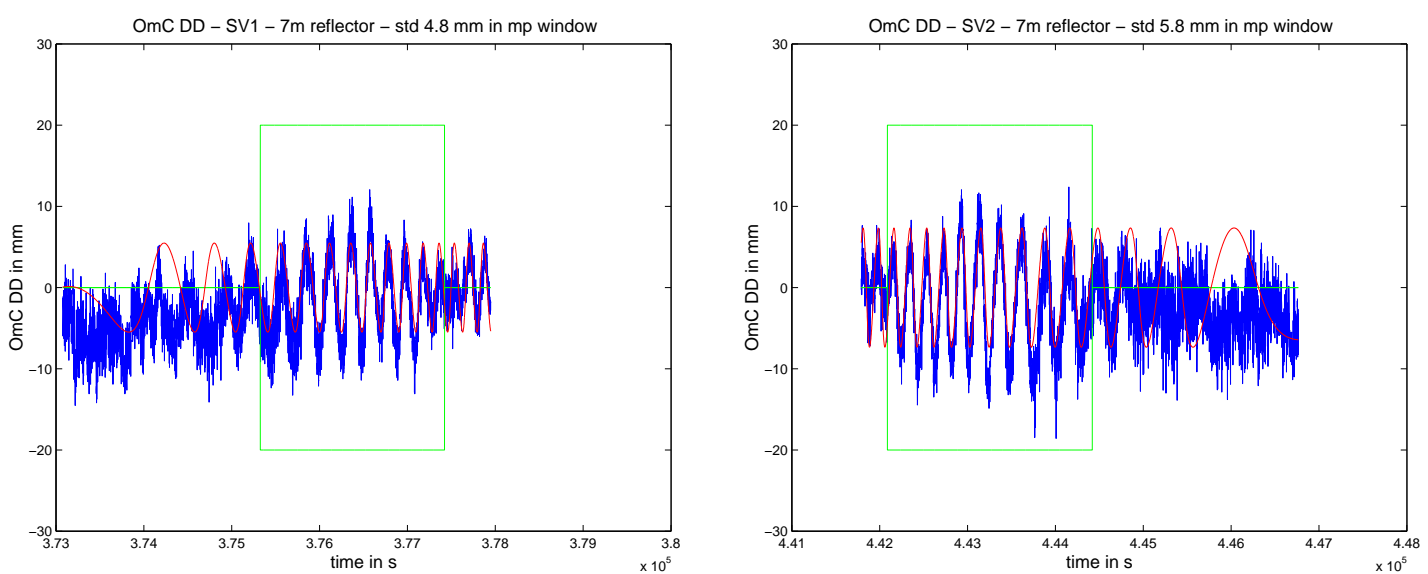

Figure 6.15c: modelled multipath phase error superimposed to the O-C DD. 
Table 6.2 summarises the optimum parameters $\alpha$ and $\Theta$ obtained, as well as the elevation and azimuth of the reflected ray (i.e. the LHCP reflected wave) and the elevation of the incident ray (i.e. the RHCP direct wave). Note that, for the incident ray, the azimuth is not shown in the table, since the antenna is considered isotropic horizontally.

\begin{tabular}{|c|c|c|c|c|c|}
\hline & $\alpha$ & $\Theta$ & $\begin{array}{c}\text { elevation of the } \\
\text { reflected ray }\end{array}$ & $\begin{array}{l}\text { azimuth of the } \\
\text { reflected ray }\end{array}$ & $\begin{array}{c}\text { elevation of the } \\
\text { incident ray }\end{array}$ \\
\hline day148 & 0.07 (SV1) & $30^{\circ}$ & $-12.5^{\circ}<\mathrm{el}<6.1^{\circ}$ & $23.9^{\circ}<\mathrm{az}<24.8^{\circ}$ & $43.3^{\circ}<\mathrm{el}<61.4^{\circ}$ \\
\hline (2002) $5 \mathrm{~m}$ & 0.12 (SV2) & $0^{\circ}$ & $-12.2^{\circ}<\mathrm{el}<12.4^{\circ}$ & $-2.6^{\circ}<\mathrm{az}<7.3^{\circ}$ & $44.9^{\circ}<\mathrm{el}<65.5^{\circ}$ \\
\hline day210 & 0.30 (SV1) & $120^{\circ}$ & $8.8^{\circ}<\mathrm{e}<43.9^{\circ}$ & $23.8^{\circ}<\mathrm{az}<27.2^{\circ}$ & $20.5^{\circ}<\mathrm{el}<55.2^{\circ}$ \\
\hline (2003) $1 \mathrm{~m}$ & 0.33 (SV2) & $90^{\circ}$ & $15.9^{\circ}<\mathrm{el}<44.5^{\circ}$ & $-0.5^{\circ}<\mathrm{az}<11.8^{\circ}$ & $27.8^{\circ}<\mathrm{el}<56.1^{\circ}$ \\
\hline day211 & 0.22 (SV1) & $120^{\circ}$ & $-18.4^{\circ}<\mathrm{el}<13.5^{\circ}$ & $23.7^{\circ}<\mathrm{az}<25.4^{\circ}$ & $27.7^{\circ}<\mathrm{el}<59.1^{\circ}$ \\
\hline (2003) $4 \mathrm{~m}$ & 0.21 (SV2) & $90^{\circ}$ & $-18.8^{\circ}<\mathrm{el}<13.7^{\circ}$ & $1.8^{\circ}<\mathrm{az}<12.6^{\circ}$ & $28.5^{\circ}<\mathrm{el}<59.7^{\circ}$ \\
\hline day212 & 0.18 (SV1) & $120^{\circ}$ & $-9.2^{\circ}<\mathrm{el}<8.3^{\circ}$ & $23.2^{\circ}<\mathrm{az}<23.9^{\circ}$ & $44.4^{\circ}<\mathrm{el}<61.4^{\circ}$ \\
\hline (2003) $7 \mathrm{~m}$ & 0.24 (SV2) & $90^{\circ}$ & $-9.2^{\circ}<\mathrm{el}<8.6^{\circ}$ & $0.7^{\circ}<\mathrm{az}<7.2^{\circ}$ & $45.6^{\circ}<\mathrm{el}<61.7^{\circ}$ \\
\hline
\end{tabular}

Table 6.2: optimum parameters $\boldsymbol{\alpha}$ and $\Theta$

These results give rise to the following comments.

The tuning of $\alpha$ and $\Theta$ is very different between the preliminary test in 2002 and the tests in 2003. This is not only due to different antennas used (same model but different serial numbers), but also (and mainly) because they are not isotropic horizontally. This is why, for orientations that were different between the two campaigns of tests, the modelling parameters (and particularly the antenna phase shift $\Theta$ ) are not the same. Actually, the antennas at base and rover where oriented roughly parallel, but not with a specific direction. 
However, within a given campaign, $\alpha$ and $\Theta$ repeat pretty well, particularly $\Theta$ because it is linked to the azimuth of the reflected ray, that varies little with the change of the conditions of tests.

The elevations of both the incident and the reflected rays vary much more from a test to another, which makes $\alpha$ change, for instance between $1 \mathrm{~m}$ test and 4 or $7 \mathrm{~m}$ tests.

For a given test and satellite, the parameters (particularly $\alpha$ ) are not optimum for the entire multipath window time period, because, within such a period, the elevations vary noticeably (the sharper the parabolic curve, the better modelling).

In the time series, multipath is clearly visible and the frequency (proportional to the additional path length), phase and amplitude of the resulting phase error correspond closely to those predicted by multipath modelling. The shorter period of phase error is observed for the reflector the farer (i.e. $7 \mathrm{~m}$ ) and it is approximately 4 minutes (see Fig. 6.15c).

It is interesting to compare these results to those that can be obtained by means of the antenna gain patterns. Fig. 6.16 shows the AT502 gain patterns, for L1 phase. The anisotropy for the LHCP reflected wave is obvious.

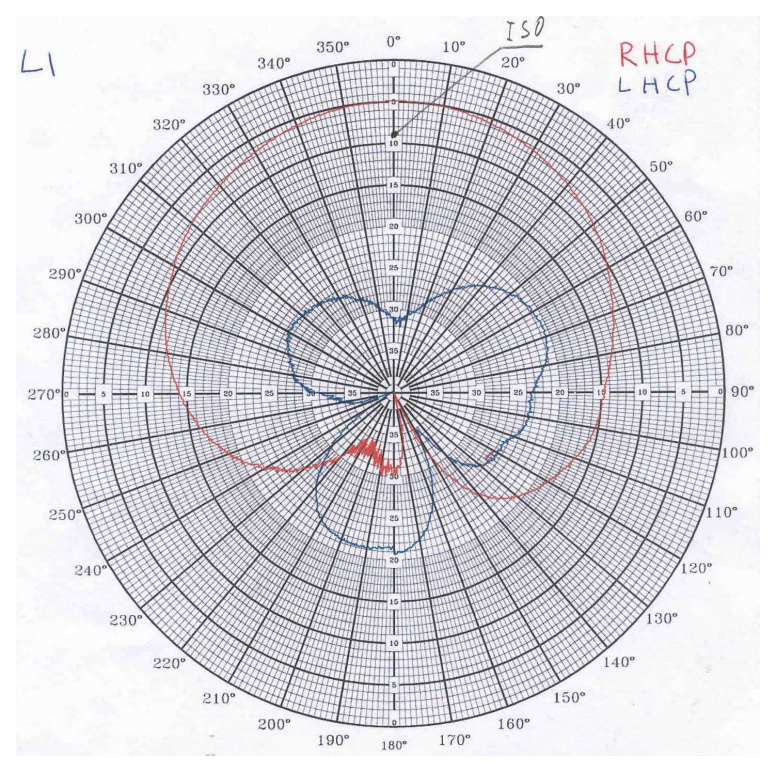

Figure 6.16: AT502 gain patterns for L1 phase (AeroAntenna) 
On the preliminary test for instance, within a multipath window, the elevation of the incident ray is $\sim 55^{\circ} \pm 10^{\circ}$, which gives $\sim-7.5 \mathrm{~dB}$ in the RHCP gain pattern. Symmetrically the elevation of the reflected ray is $\sim 0^{\circ} \pm 10^{\circ}$, which respectively gives $\sim-21.5 \mathrm{~dB}$ and $\sim-30.5 \mathrm{~dB}$ gains whether the positive or negative lobes of the LHCP gain pattern are considered. So, the corresponding values of $\alpha$ are respectively: $10^{\wedge(7.5-21.5) / 20)}=0.20$ and: $10^{\wedge(7.5-30.5 / 20)}=0.07$.

The preliminary test was with the panel at $5 \mathrm{~m}$. It can be compared to the test at $4 \mathrm{~m}$ (that gives about the same range of additional path length). For this one, the elevation of the incident ray is $\sim 45^{\circ} \pm 15^{\circ}$, which gives $\sim-8.5 \mathrm{~dB}$ in the RHCP gain pattern. Symmetrically the elevation of the reflected ray is $\sim-5 \pm 15^{\circ}$, which respectively gives $\sim-22 \mathrm{~dB}$ and $\sim-31.5 \mathrm{~dB}$ gains whether the positive or negative lobes of the LHCP gain pattern are considered. So, the corresponding values of $\alpha$ are respectively. $10^{\wedge(8.5-22) / 20)}=0.21$ and: $10^{\wedge(8.5-3-31.5 / 20)}=0.07$.

Note that these values of $\alpha$ are limited to a certain angular domain within the antenna RHCP and LHCP gain patterns, and cannot be generalised to all possible relative geometries of the antenna and the reflector.

The values of $\alpha$ for the two tests considered here (i.e. the preliminary test at $5 \mathrm{~m}$ and the test at $4 \mathrm{~m}$ ) are totally coherent with those obtained in the tuning process described before in this section. They illustrate that the orientation of the AT502 antenna during the experiment is one of the most important conditions of tests. It actually generates multipath phase error with an amplitude that may change by up to a factor of 3 , since the LHCP gain pattern is particularly not isotropic in azimuth. Since gain and phase patterns are linked, this also explains why the antenna phase shift is singularly different between the different tests. 


\section{3 - Results of static tests}

Note: only the second campaign of tests is considered to assess the PMMW technique.

A summary of the static test programme has been given in table 6.1. The static tests duration was 23 hours. The log files have been split into 3 parts: 8.00-16.00 h, 16.00-0.00 h next day, and 0.00-8.00 h, for easier computer processing (see e.g. Fig. 6.18 for day211, 8.00-16.00 h), in GPS time.

In the analysis, every satellite is selected in turn, and its position relative to the reflector and the rover antenna is checked to identify multipath, by means of the method presented in §6.2. Time series of Observed-Computed (O-C) Double Differences (DD) of L1 phase "standard" measurements are displayed in Fig. 6.18, as well as the "green windows" delimitating the multipath time zones and the "red windows" delimitating the diffraction time zones. Fig. 6.17 recalls schematically the meaning of these zones. As DD are represented, the gaps that appear in the time series correspond to when the considered satellite is the differencing one. The elevation of the satellites are also superimposed.

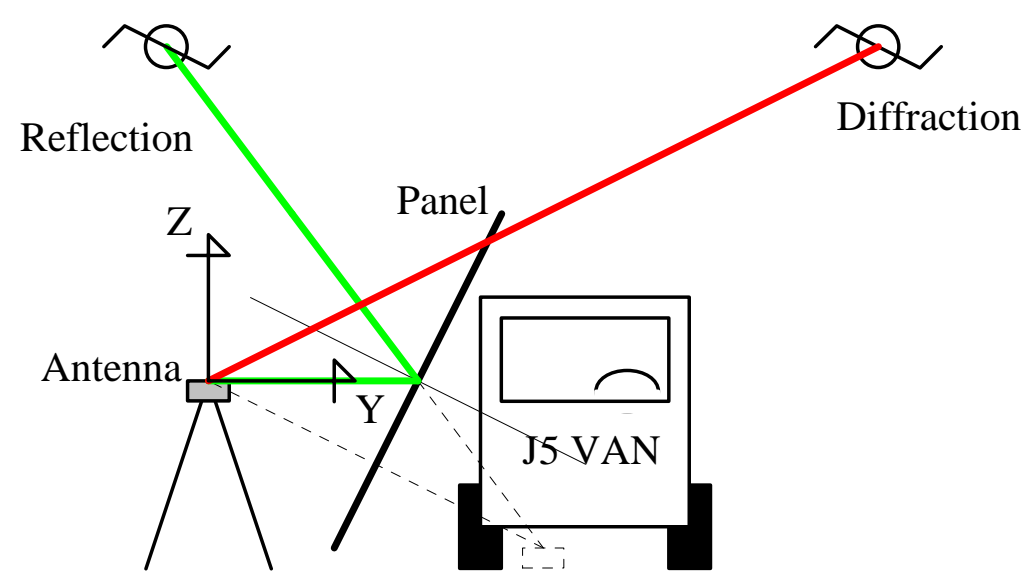

Figure 6.17: reflection and diffraction geometrical computation

The O-C DD rely on the known position of the rover given by a GPS static survey of the tripod from one of the SESSYL monuments (B2). It was done just after the campaign, during day 213 . 

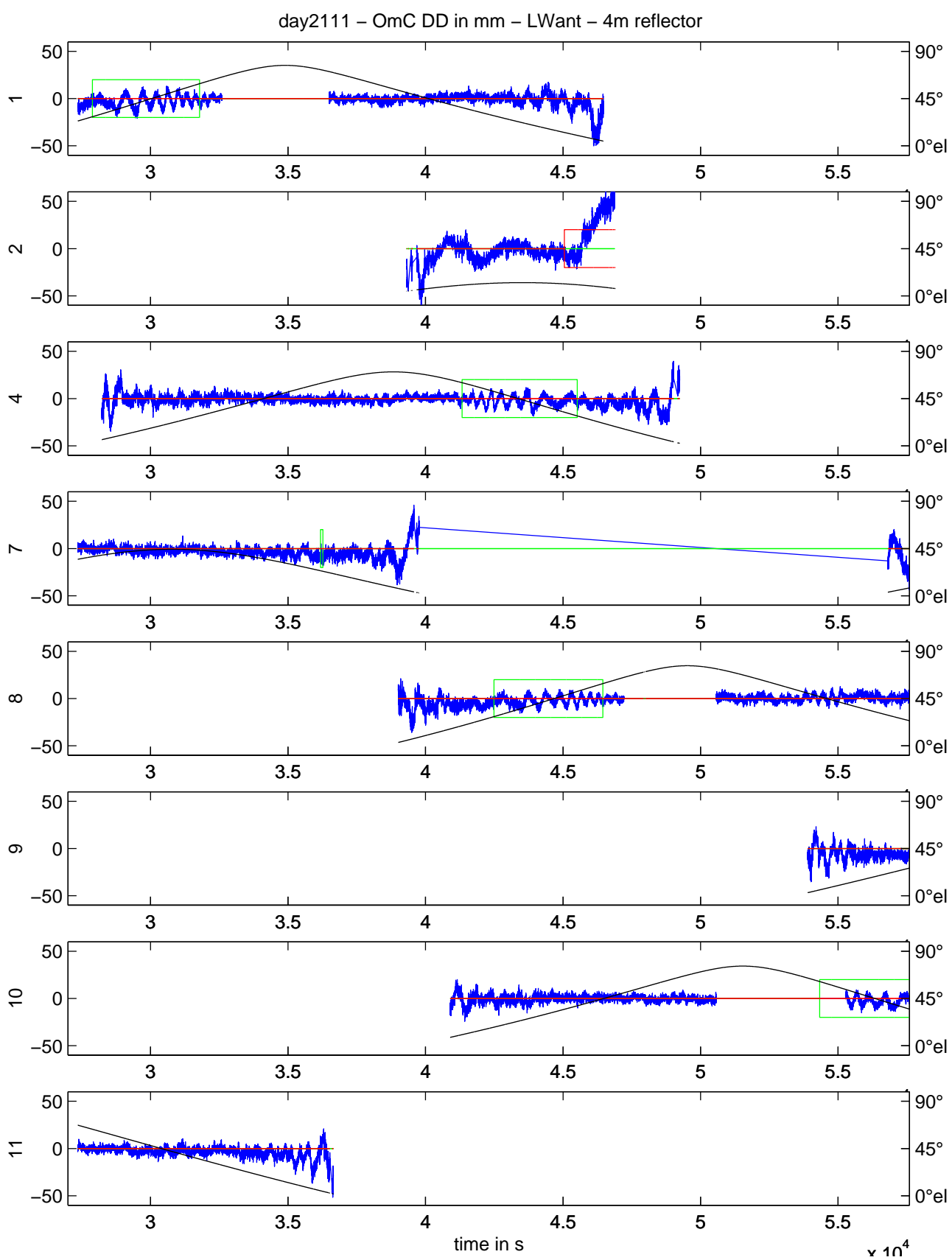

Figure 6.18: static test with the panel at $4 \mathrm{~m}$ (8 SVs displayed only) - PMMW corrections disabled

Fig. 6.18 shows O-C DD of L1 phase "standard" measurements, i.e. measurements that are not corrected by the PMMW outputs. The analysis was duplicated (see Fig. 6.19) applying PMMW corrections at both base and rover as specified in Eq. 5.1. 

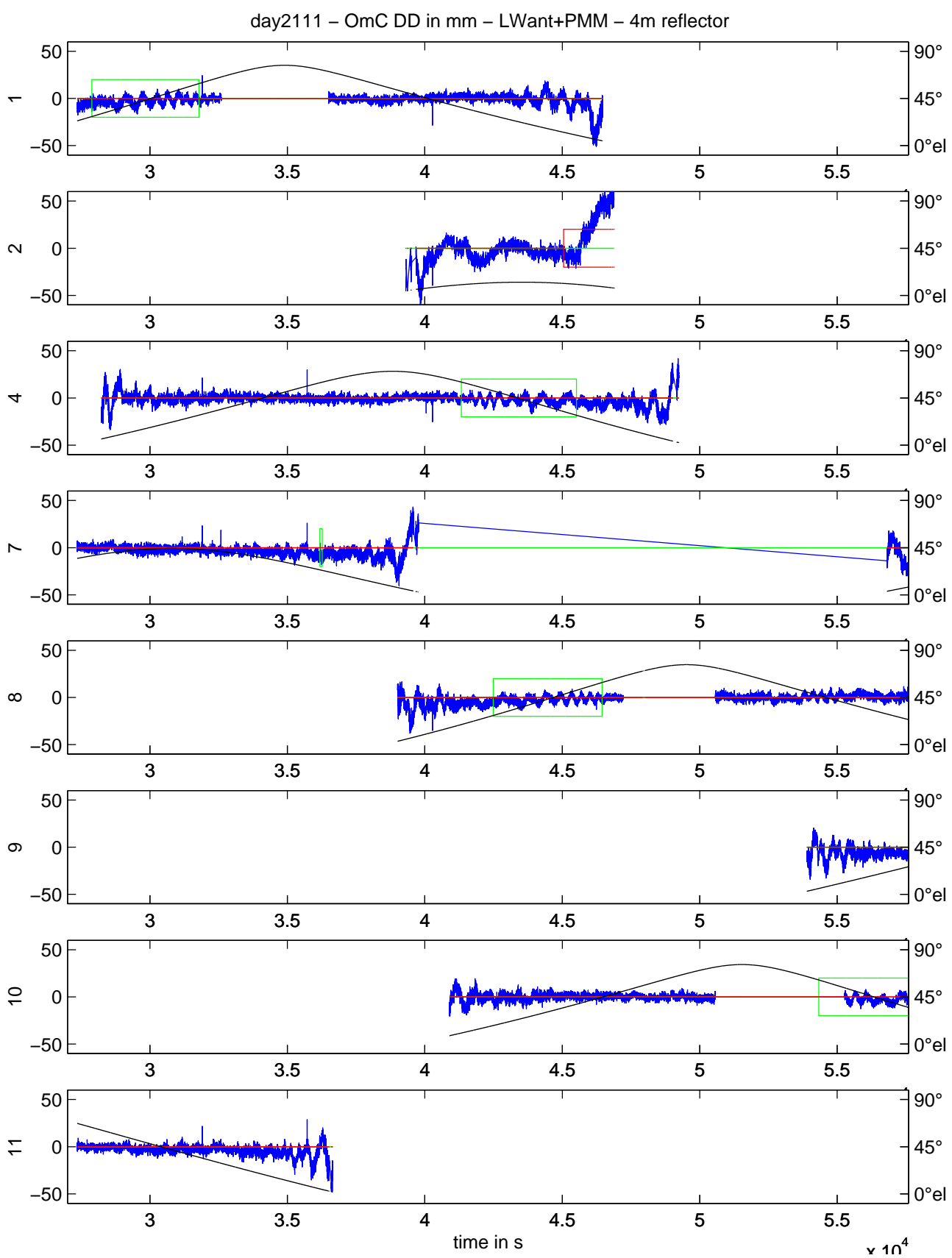

Figure 6.19: static test with the reflector at $4 \mathrm{~m}$ (8 SVs displayed only) - PMMW corrections enabled

Fig. 6.20a to 6.20c focus on SV1 and SV2 "green windows" and display together (and for each day) O-C DD of L1 phase without and with PMMW corrections. A few aberrant PMMW corrections are visible (peaks in O-C DD of L1 phase). 

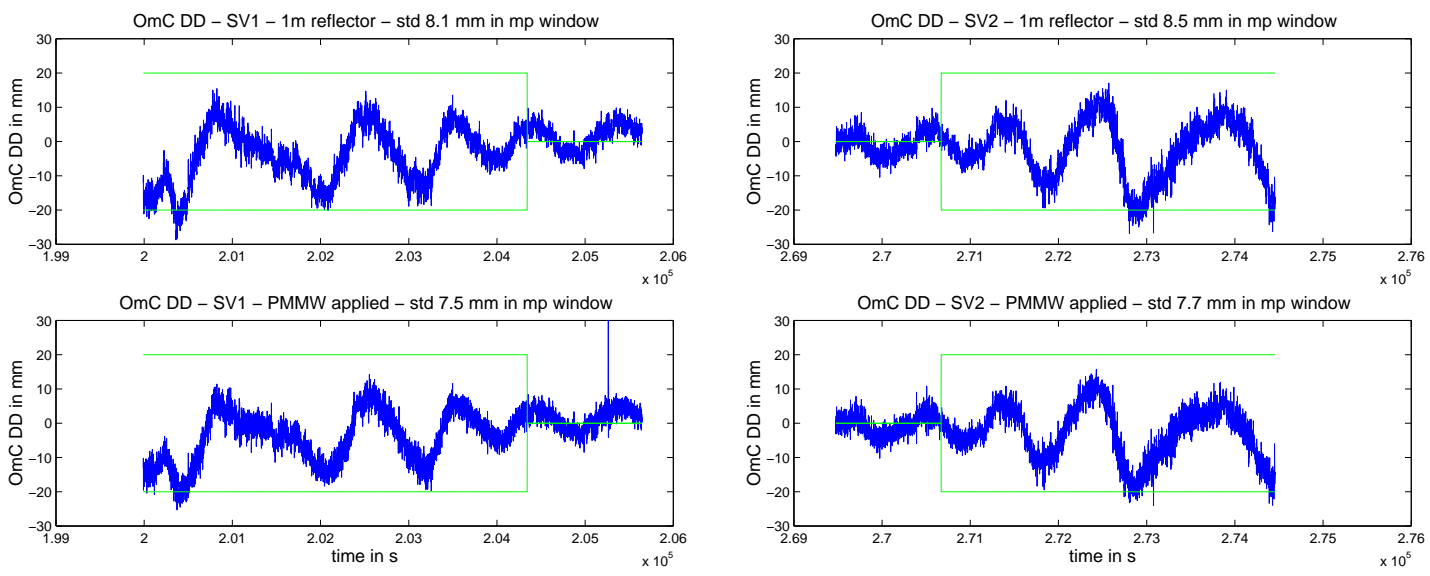

Figure 6.20a: effect of PMMW on phase DD in static mode (day210 - panel at $1 \mathrm{~m}$ )
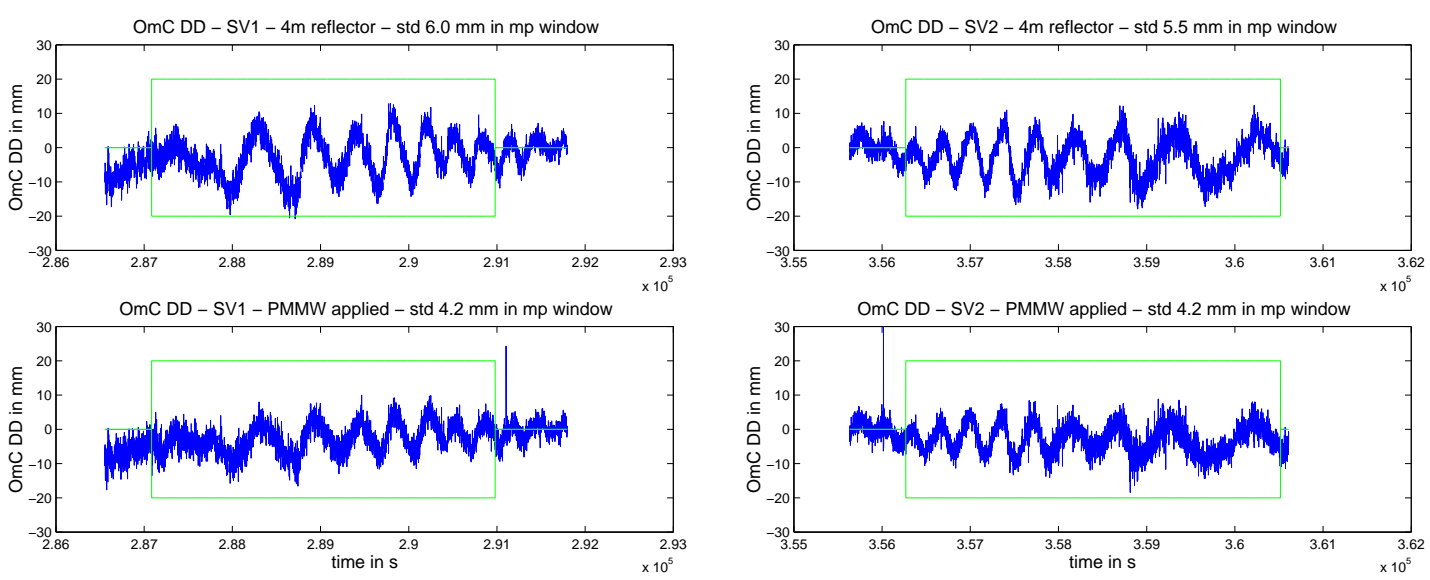

Figure 6.20b: effect of PMMW on phase DD in static mode (day211 - panel at $4 \mathrm{~m}$ )
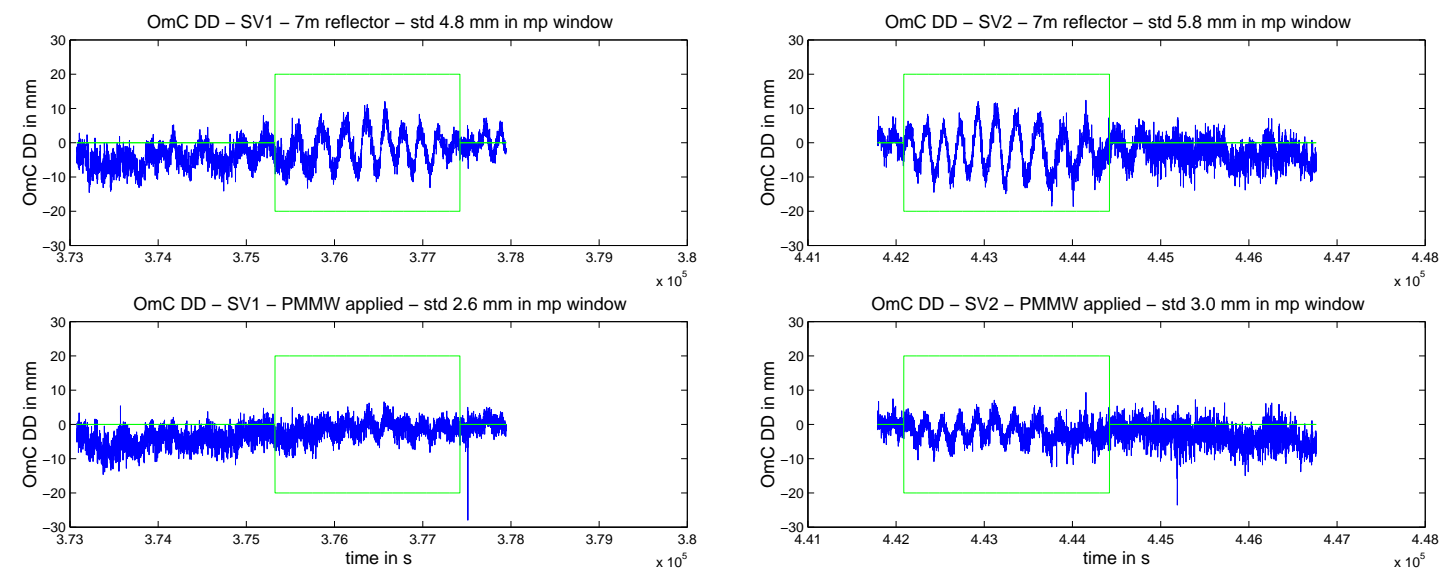

Figure 6.20c: effect of PMMW on phase DD in static mode (day212 - panel at $7 \mathrm{~m}$ ) 
Note that Fig. 6.20b is a zoom on the first upper time series in Fig. 6.18 and 6.19.

The analysis consists in computing for each satellite the corresponding standard deviation of the Observed-Computed DD of L1 phase within its multipath window time zone, and repeat this twice (i.e. without the PMMW corrections, and again with).

In the meantime, the same computation is done within the equivalent time zone during day209 (taking care of shifting by 3'56" per day to compensate the duration of the sidereal day). That day, no panel perturbed the measurement process. It was checked that data logged that day can be considered as clean in terms of multipath and local ionospheric effects. Day209 statistics set up a reference and give an idea of the influence of the reflector. Moreover, they enable the assessment of the PMMW process on clean data.

Tables 6.3a, 6.3b and 6.3c correspond respectively to day 210 , day211 and day 212 . Two gains (the one for the data logged during the day that is considered, and the other for the reference day, i.e. day209) are computed as follows:

gain $($ in $\%)=100 *\left(\sigma-\sigma_{\mathrm{PMMW}}\right) / \sigma$

where:

$\sigma$ is the standard deviation of the O-C DD of L1 phase, that means here "standard" L1 phase;

$\sigma_{\mathrm{PMMN}}$ is the standard deviation of the O-C DD of PMMW "corrected" L1 phase.

Note that if ever a satellite becomes the reference satellite for double differencing for part of its multipath window time zone, the corresponding standard deviations and gains do not include the epochs when this is the reference satellite (during this time the O-C DD are null, and they would bias the statistics). This occurs for SV10 as it is a relatively low elevation reference satellite. 
Legend: sv satellite number

dura duration of the multipath window time zone in $\mathrm{s}$

mind minimum additional path length in $\mathrm{m}$

maxd maximum additional path length in $\mathrm{m}$

avgd average additional path length in the multipath window time zone in $\mathrm{m}$

For a given day of test:

sd_ standard deviation of the O-C DD of "standard" L1 phase in mm

sdx standard deviation of the O-C DD of PMMW "corrected" L1 phase in mm corresponding gain in $\%$

For the reference day of test (day209):

rf_ standard deviation of the O-C DD of "standard" L1 phase in mm

rfx standard deviation of the O-C DD of PMMW "corrected" L1 phase in mm corresponding gain in \%

non* part in s of the multipath window time zone when SV10 is not differencing

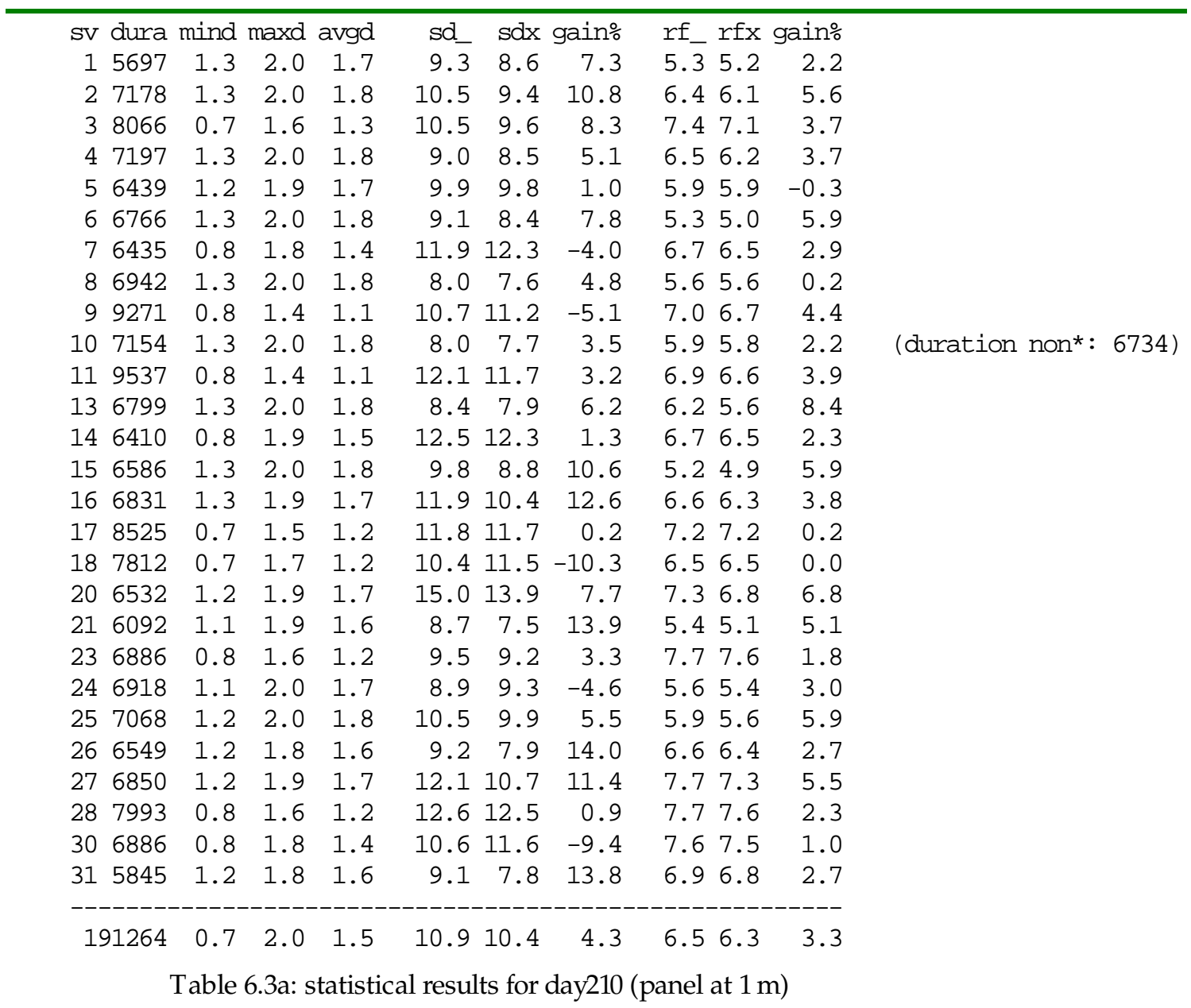




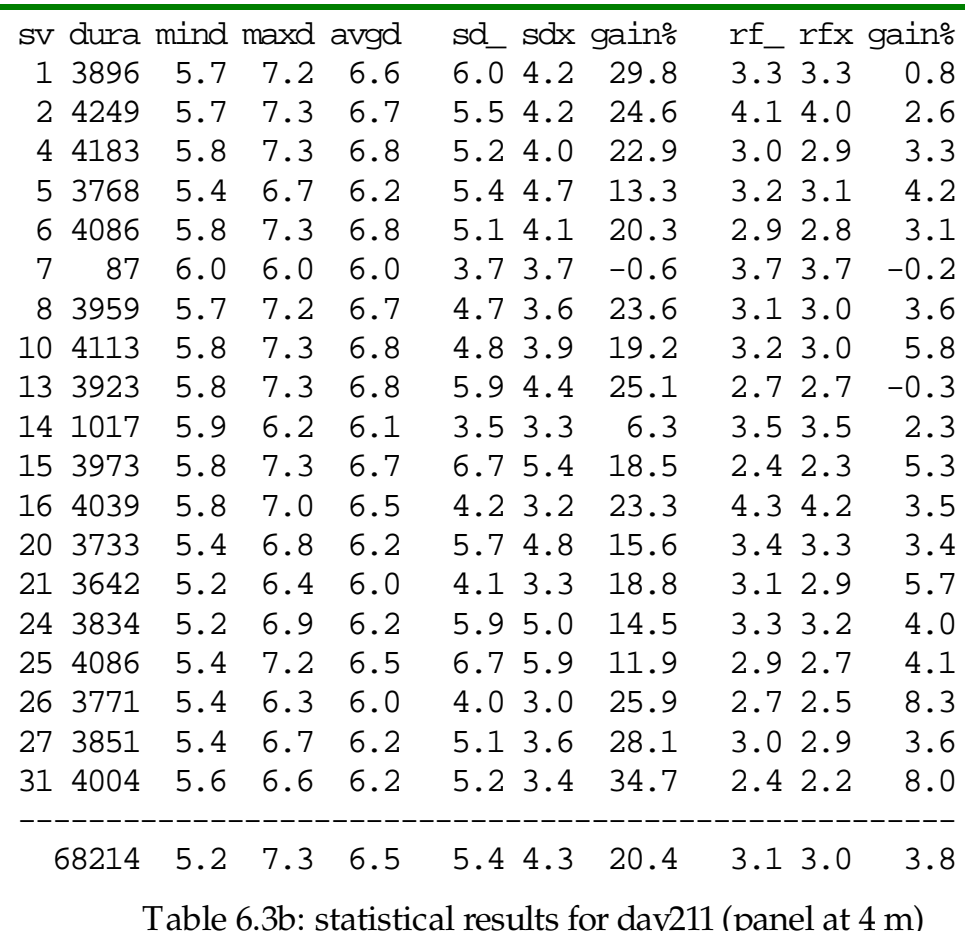

(duration non*: 3172)

Table 6.3b: statistical results for day211 (panel at $4 \mathrm{~m}$ )

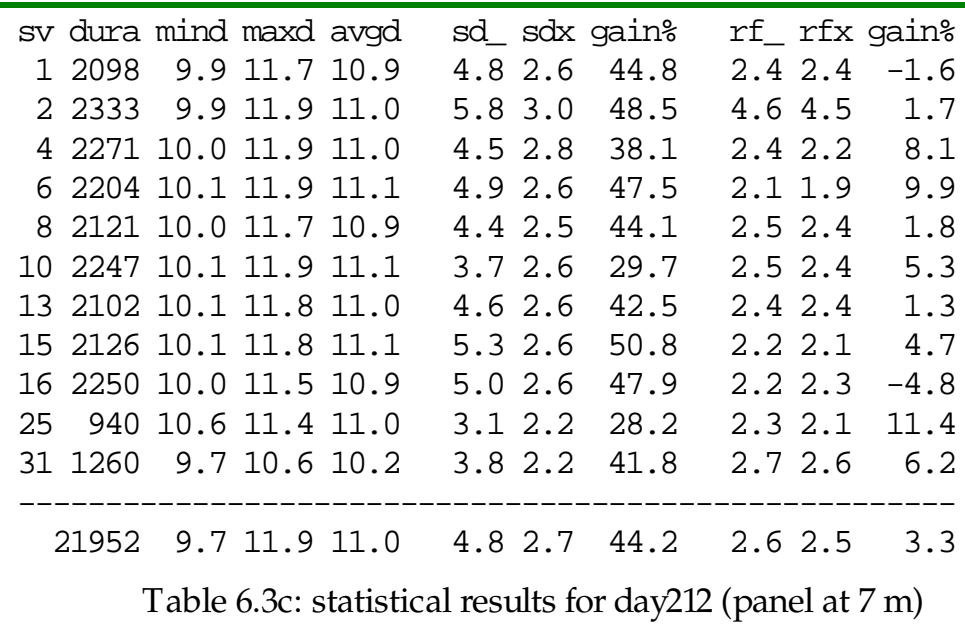

The results in static mode are summarised in table 6.4 (as well as in the lower line of tables $6.3 \mathrm{a}$ to $6.3 \mathrm{c}$ ). Table 6.4 gives statistics generalised to all satellites that enter the multipath zones and similarly in the equivalent time zones for the test with no reflector (this is displayed in italic). 


\begin{tabular}{|c|c|c|c|}
\hline Static tests & $\operatorname{Std} \sigma$ & Std $\sigma_{\mathrm{PMMW}}$ & Gain \\
\hline $\begin{array}{c}\text { reflector } 1 \mathrm{~m} \\
0.7 \mathrm{~m}<\text { addpath }<2.0 \mathrm{~m}\end{array}$ & $10.9 \mathrm{~mm}$ & $10.4 \mathrm{~mm}$ & $4 \%$ \\
\hline Noreflector & $6.5 \mathrm{~mm}$ & $6.3 \mathrm{~mm}$ & $3 \%$ \\
\hline $\begin{array}{c}\text { reflector } 4 \mathrm{~m} \\
5.2 \mathrm{~m}<\text { addpath }<7.3 \mathrm{~m}\end{array}$ & $5.4 \mathrm{~mm}$ & $4.3 \mathrm{~mm}$ & $20 \%$ \\
\hline Noreflector & $3.1 \mathrm{~mm}$ & $3.0 \mathrm{~mm}$ & $4 \%$ \\
\hline $\begin{array}{c}\text { reflector } 7 \mathrm{~m} \\
9.7 \mathrm{~m}<\text { addpath }<11.9 \mathrm{~m}\end{array}$ & $4.8 \mathrm{~mm}$ & $2.7 \mathrm{~mm}$ & $44 \%$ \\
\hline Noreflector & $2.6 \mathrm{~mm}$ & $2.5 \mathrm{~mm}$ & $3 \%$ \\
\hline
\end{tabular}

Table 6.4: L1 phase DD statistics in static mode

The level of the phase error $(6.5 \mathrm{~mm})$, without the panel, when the panel would have been at $1 \mathrm{~m}$ is twice that when it would have been at 4 or $7 \mathrm{~m}(3.1$ and $2.6 \mathrm{~mm})$. This is due to the fact that the time zones used include satellites with much lower elevation for this test than for the others (see Fig. 6.10): so measurements are noisier. Besides this noise, the level of the multipath phase error $(10.9 \mathrm{~mm})$ is also much greater with the panel placed very close to the antenna. This was already underlined when tuning the modelled parameter $\alpha$.

It also appears that for the test with the panel at $1 \mathrm{~m}$ several satellites have "negative" gains, which means that the PMMW damages the measurements instead of correcting them. Generally, these satellites correspond to the smallest additional path lengths and they have also relatively low elevation.

The main conclusion of the static tests is that the PMMW correlator improved the multipath affected phase measurements by up to $44 \%$. This result was obtained with a reflector sufficiently far away (additional path length greater than $7.5 \mathrm{~m}$ ) and with a lightweight antenna.

Note: during the preliminary campaign of tests in 2002, it was shown for a choke-ring antenna that the improvement was rather half that for a lightweight [BÉTAILLE et al., 
2003-1]. Results are given in Appendix 4. The level of improvement for the PMMW technique is much greater on a signal measured by a lightweight antenna than by a chokering, since a choke-ring mitigates much of the multipath via its antenna design. Moreover, a reason why it is particularly interesting to focus on lightweight antennas is because these are much more common on worksites and in the applications envisaged in this research.

Table 6.4 also confirms the expected main result: the PMMW efficiency is theoretically and effectively dependent on the additional path length travelled by the reflected signals. The threshold around $7.5 \mathrm{~m}$ is confirmed (i.e. 1/40th of a chip length for the $40 \mathrm{MHz}$ clocked implementation in the Leica System 500 receivers).

Another conclusion comes from the statistics (given as a reference) when there is no reflector. It is especially noticeable that there is an improvement of a few $\%$ when there is no reflector. This can be explained by the ability of the phase window correlator to mitigate even weak multipath that exists in the general environment.

Lastly, it is also interesting to notice that the PMMW correlator does not address the diffraction mitigation at all (see Fig. 6.21).

Note that Fig. 6.21 is a zoom on the second upper time series in Fig. 6.18 and 6.19.
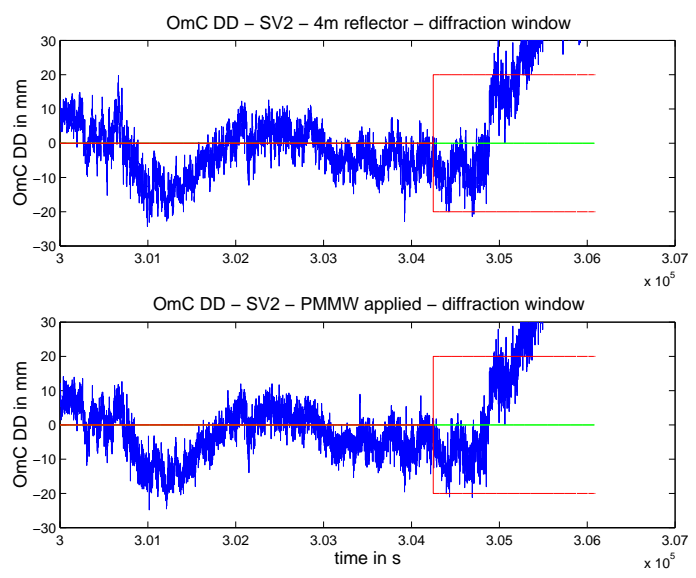

Figure 6.21: unability of PMMW to mitigate diffraction 


\section{4 - Results of kinematic tests}

Note: only the second campaign of tests is considered to assess the PMMW technique.

The preliminary campaign included a few kinematic tests that mainly showed that the SESSYL metallic plate was causing multipath, additionally to the panel (see Appendix 5). This has been efficiently mitigated by protecting the antenna with a foam that covers the SESSYL plate.

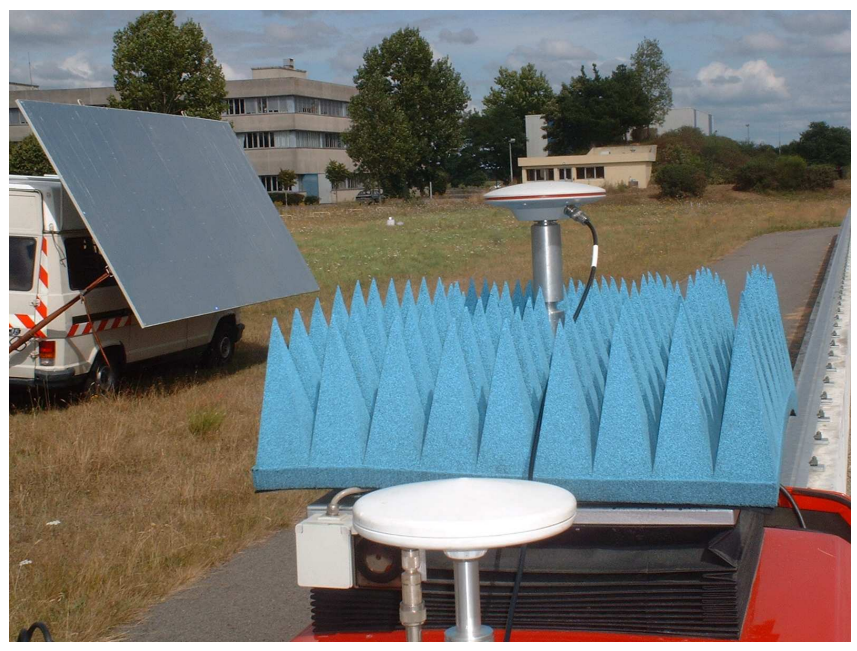

Figure 6.22: the SESSYL platform, covered by the foam, and the panel at $8 \mathrm{~m}$

Note: the second antenna visible on Fig. 6.22 is connected to another GPS receiver connected to SESSYL and dedicated to the delivery of a Pulse Per Second in order to date the SESSYL reference trajectory in GPS time (i.e. UTC time by means of the PPS + $13 \mathrm{~s}$ ).

Table 6.5 shows the planning of the kinematic tests.

\begin{tabular}{lcccc}
\hline Campaign in 2003 & day203 & day204 & day205 & day206 \\
Reflector & at $1 \mathrm{~m}, 6^{\circ}$ tilt & at $4 \mathrm{~m}, 24^{\circ}$ tilt & at $7 \mathrm{~m}, 28^{\circ}$ tilt & no \\
\hline
\end{tabular}

Table 6.5: kinematic tests planning and conditions of tests 
The SR530 receivers and AT502 antennas used were the same as for the static tests. The kinematic tests are series of the same and repeated SESSYL cycle, whose duration was 20 minutes, and that consisted in performing the first straight line (where the $5 \mathrm{~m} \times 2.5 \mathrm{~m}$ panel was set-up) at a speed of $0.05 \mathrm{~m} / \mathrm{s}$ and returning automatically at high speed $(1 \mathrm{~km} / \mathrm{h})$. The SESSYL reference trajectory (that enable the O-C DD of L1 phase to be computed) is available only during the front pass, not during the return pass. Hence, only the first pass could be used effectively in the testing methodology. The time spent in front of the panel was around $100 \mathrm{~s}$ at $0.05 \mathrm{~m} / \mathrm{s}$. 27 of these tests were carried out each day (but they were stopped at night). Data logging was stopped every two or three hours, as follows: 5.40-7.40 h, 1 hour pause, 8.40-10.40 h (see e.g. Fig. 6.23 for day203) immediately followed by 10.40-13.40 h, 1 hour pause, and 14.40-16.40 h, in GPS time.

Note that the kinematic tests with SESSYL started at the same sidereal time each day, in order to keep the same constellation at the antenna locations and so maintain identical geometry between the tests.

The SESSYL reference trajectory, i.e. the co-ordinates of the rover antenna, are known in 3 dimensions with an accuracy of $1 \mathrm{~mm}(1 \sigma)$ when SESSYL is moving at a speed of $0.05 \mathrm{~m} / \mathrm{s}$ [BÉTAILLE et al., 2000].

The same analysis as that presented for the static data sets can be done for the kinematic data sets: computation of O-C DD of L1 phase, determination of the multipath and diffraction windows, graphical and statistical presentations of the results. Note that the given standard deviation of the O-C DD of L1 phase may not represent the exact size of the multipath phase error, because this standard deviation is computed on relatively short multipath window time zones, within which these DD may be locally biased (i.e. not centred around zero). 

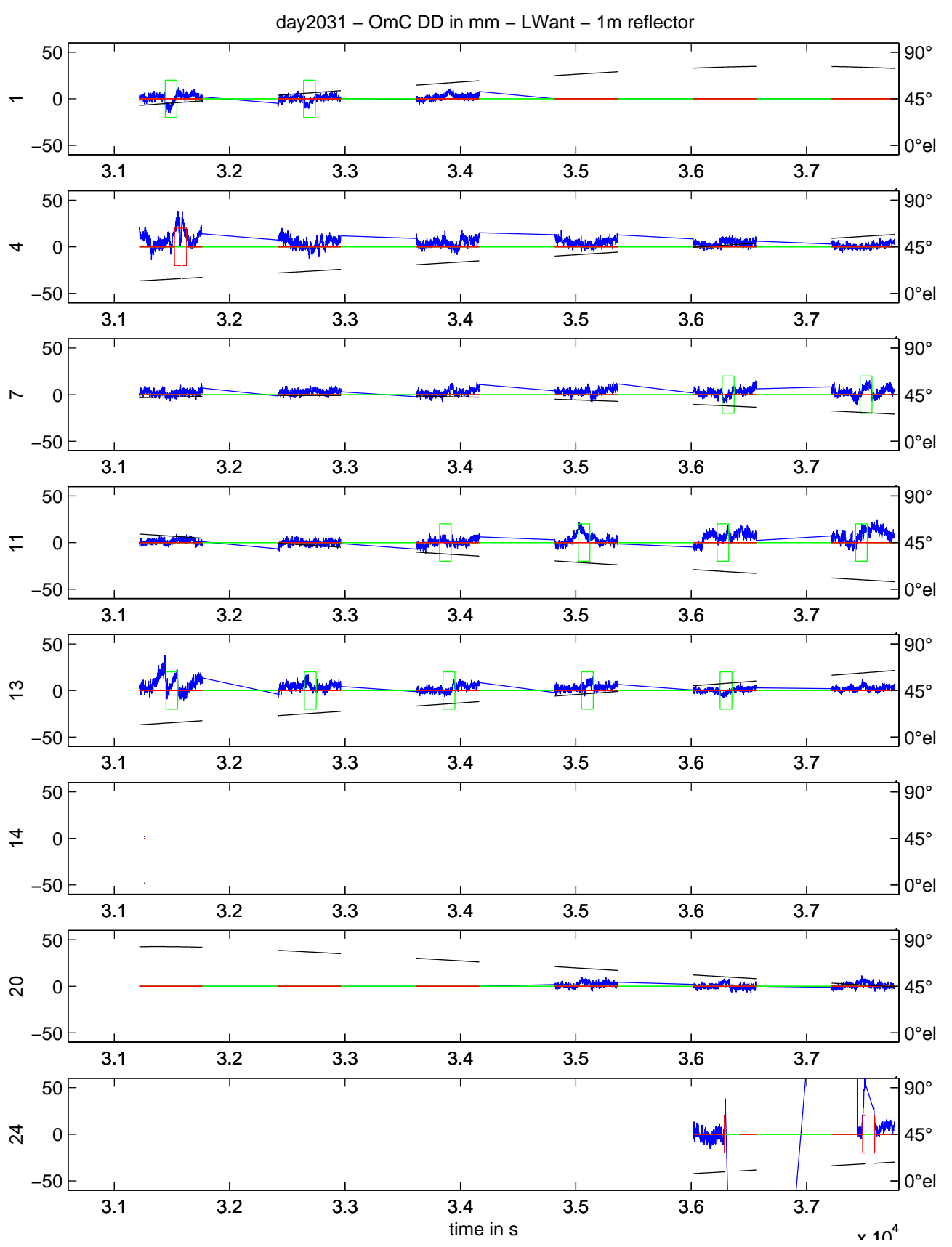

Figure 6.23: kinematic test with the panel at $1 \mathrm{~m}$ (8 SVs displayed only) - PMMW disabled

Fig. 6.23 shows O-C DD of L1 phase "standard" measurements, i.e. measurements that are not corrected by the PMMW outputs. The analysis was duplicated (see Fig. 6.24) applying PMMW corrections at both base and rover as specified in Eq. 5.1. 

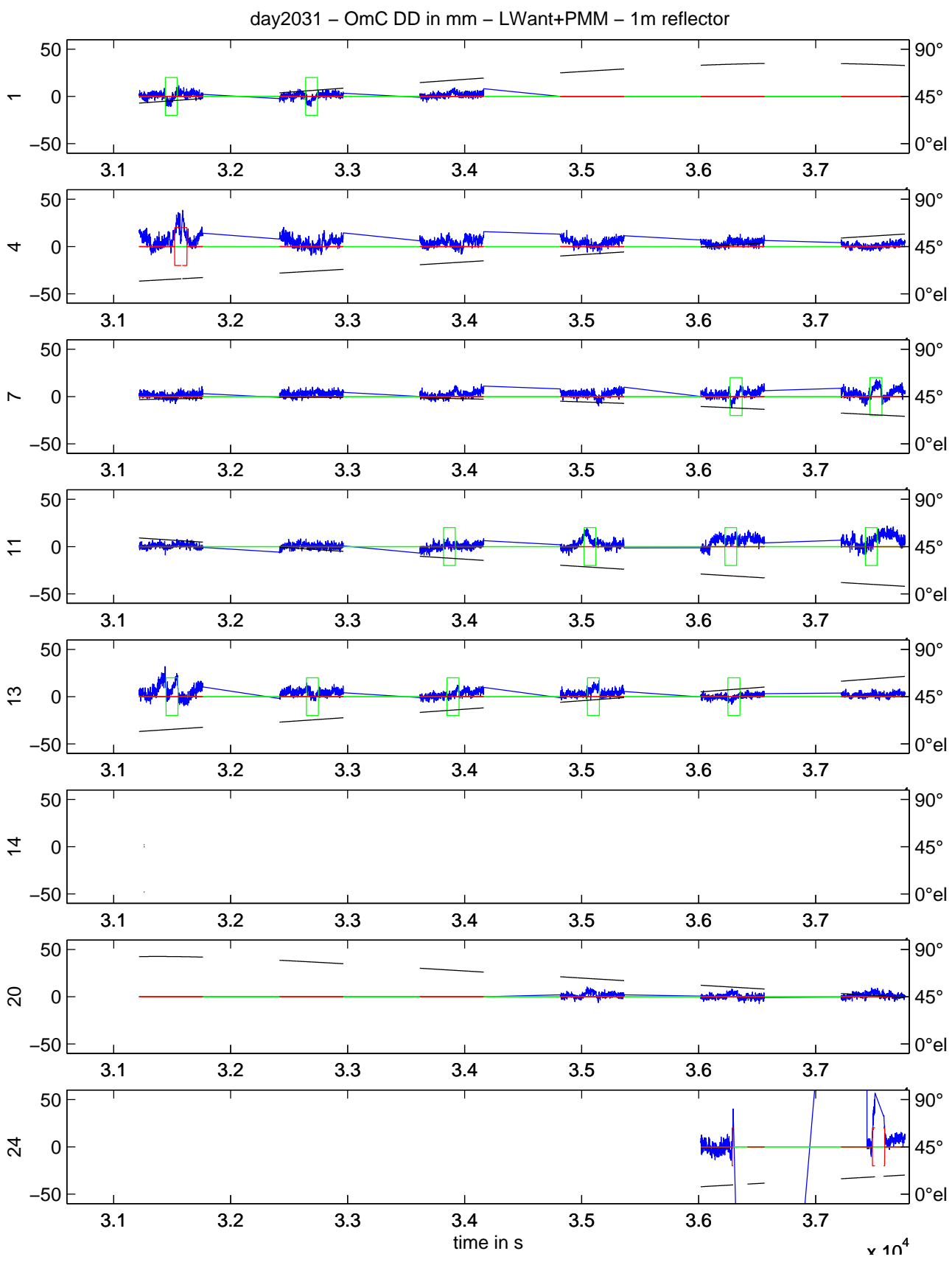

Figure 6.24: kinematic test with the reflector at $1 \mathrm{~m}$ (8 SVs displayed only) - PMMW enabled 
Table 6.6a, 6.6b and 6.6c correspond respectively to day203, day204 and day205. The reference day for the kinematic tests is day206. The statistics given are the same as in table 6.3. The duration of the multipath window time zones results of the concatenation of several tests, so it is often near a multiple of $100 \mathrm{~s}$, except when a satellite reflects near the edge of the panel and may get out this window while SESSYL passes in front of the panel.

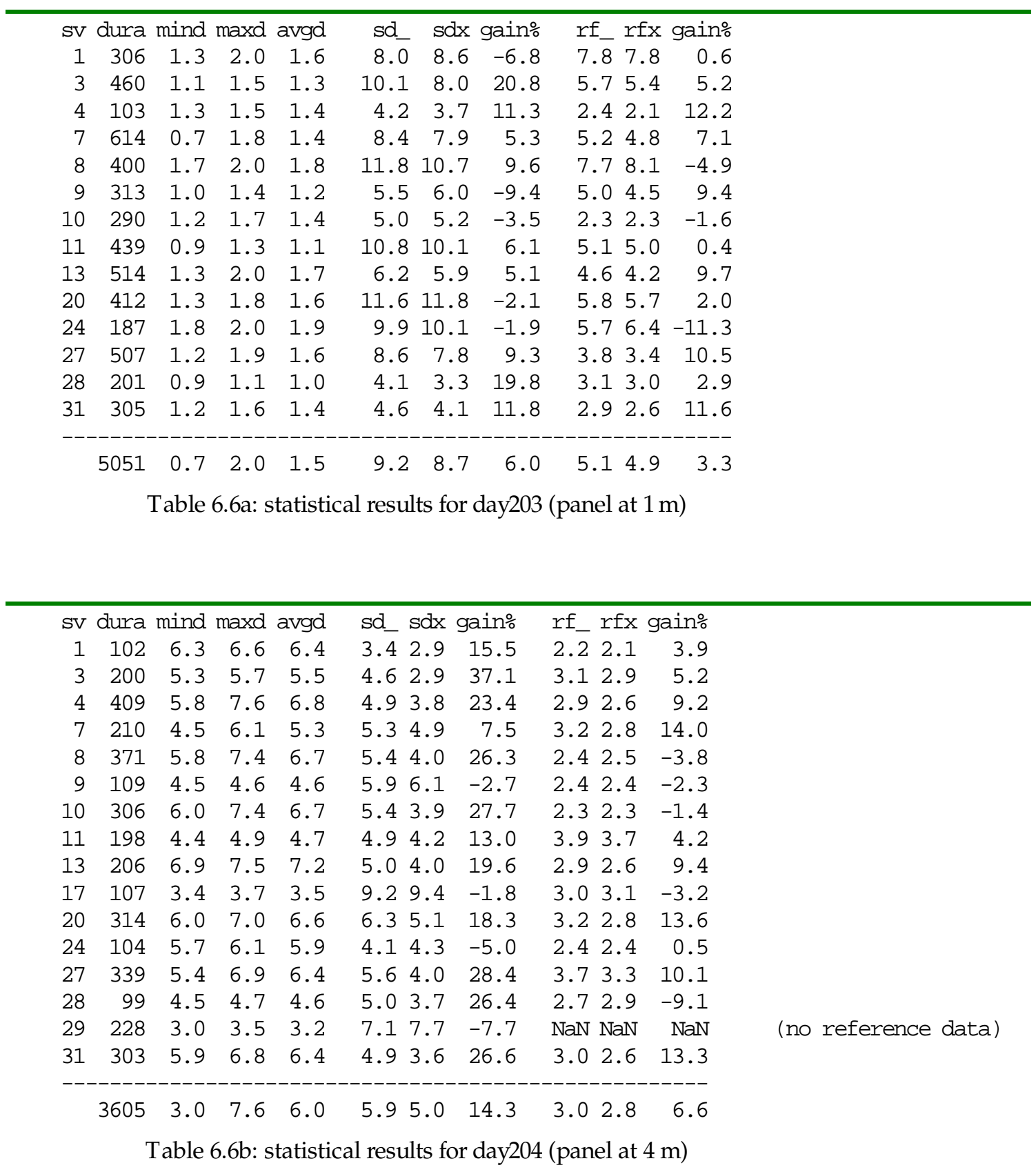




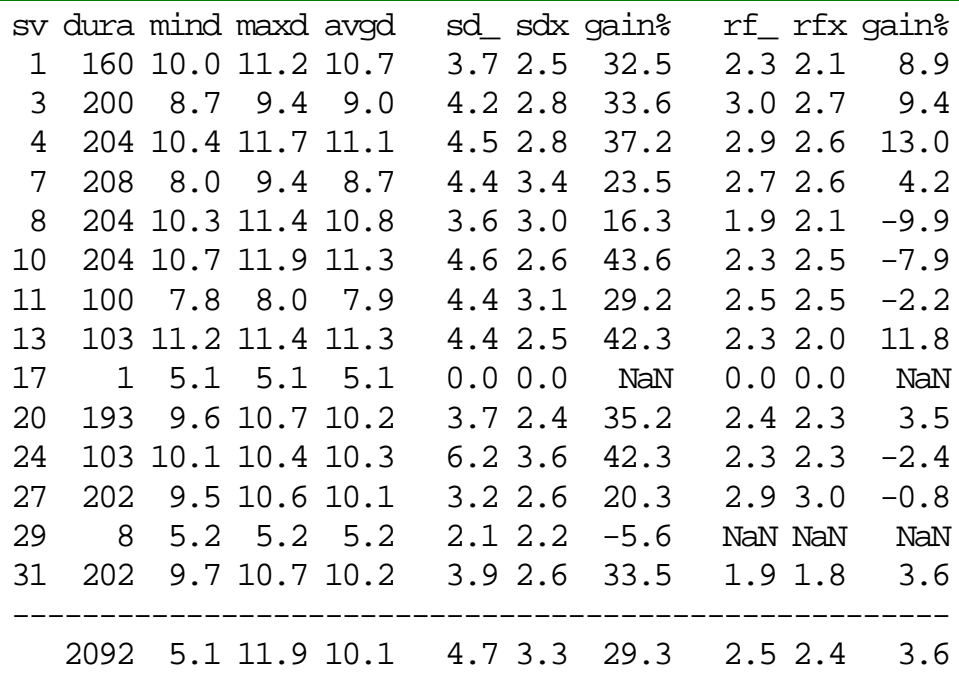

Table 6.6c: statistical results for day205 (panel at $7 \mathrm{~m}$ )

The kinematic results are summarised in table 6.7 (as well as in the lower line of tables 6.6a to $6.6 \mathrm{c}$ ). Table 6.7 gives statistics generalised to all satellites that enter the multipath zones and similarly in the equivalent time zones for the test with no reflector (this is displayed in italic). They show the same trend as in the static case, although in this case the phase window correlator is slightly less efficient.

\begin{tabular}{|c|c|c|c|}
\hline Kinematic tests & Std $\sigma$ & Std $\sigma_{\mathrm{PMMW}}$ & Gain \\
\hline $\begin{array}{c}\text { reflector } 1 \mathrm{~m} \\
0.7 \mathrm{~m}<\text { addpath }<2.0 \mathrm{~m}\end{array}$ & $9.2 \mathrm{~mm}$ & $8.7 \mathrm{~mm}$ & $6 \%$ \\
\hline Noreflector & $5.1 \mathrm{~mm}$ & $4.9 \mathrm{~mm}$ & $3 \%$ \\
\hline $\begin{array}{c}\text { reflector } 4 \mathrm{~m} \\
3.0 \mathrm{~m}<\text { addpath }<7.6 \mathrm{~m}\end{array}$ & $5.9 \mathrm{~mm}$ & $5.0 \mathrm{~mm}$ & $14 \%$ \\
\hline Noreflector & $3.0 \mathrm{~mm}$ & $2.8 \mathrm{~mm}$ & $7 \%$ \\
\hline $\begin{array}{c}\text { reflector } 7 \mathrm{~m} \\
5.1 \mathrm{~m}<\text { addpath }<11.9 \mathrm{~m}\end{array}$ & $4.7 \mathrm{~mm}$ & $3.3 \mathrm{~mm}$ & $29 \%$ \\
\hline Noreflector & $2.5 \mathrm{~mm}$ & $2.4 \mathrm{~mm}$ & $4 \%$ \\
\hline
\end{tabular}

Table 6.7: L1 phase DD statistics in kinematic mode 


\section{5 - Conclusions}

The campaign of tests carried out at the LCPC showed that the patented phase MMW technique, as implemented in the Leica System 500 receiver, always improved GPS phase measurements. It did this by:

- reducing the noise of the phase measurements (by a few \%) - so leading to an improvement irrespective of the presence of the reflector (there is never a multipath free environment); and

- significantly reducing the impact of multipath as long as the additional path length of the reflected signal was at least $7.5 \mathrm{~m}$.

In the static tests, the phase MMW correlator improved the multipath affected phase measurements by nearly $50 \%$ for lightweight antennas with a reflector sufficiently far away (additional path length greater than $7.5 \mathrm{~m}$ ), and about half this for choke-ring antennas.

The results of the kinematic tests are harder to interpret as the time periods of multipath occurrence are of a rather short duration. The results do, however, indicate that the application of the phase MMW correlator leads to significant improvements in the measurements. The incidence of the reflecting panel on the measurements was clearly visible when the lightweight antenna was used and the correlator improved the phase measurements by of the order of $30 \%$ (with a reflector sufficiently far away).

The fact that the mitigation was not effective for close reflectors was confirmed by the results of applying the phase MMW correlator when the panel was at a distance of only $1 \mathrm{~m}$ (and also $4 \mathrm{~m}$ but to a lesser extent) in both the static and kinematic tests. It appears that for an additional distance of $7.5 \mathrm{~m}$ or less, the method actually does not result in such high gains. In other words the test campaign has confirmed the theory that the phase window correlator is unable to mitigate significantly multipath that is due to reflectors very close to the antenna. 


\section{Chapter 7}

\section{IMPROVING THE PHASE MMW \\ BY USING MULTIPATH FUNCTIONAL MODELING}

The objective of the investigations reported in this chapter is to overcome a key limitation of the PMMW technique. This is that it appears that for additional distances of $7.5 \mathrm{~m}$ or less, which corresponds to the $40 \mathrm{MHz}$ clock rate of Leica receivers, the improvement obtained by this technique progressively decreases until being not that significant (note that this limitation is supported by theory of the method). Chapter 7 suggests an algorithm that might deliver a significant improvement, irrespective of the distance between the rover antenna and the reflector.

\section{1 - Approximation of the multipath modelling theory}

\subsection{1 - Phase error}

The carrier phase tracking loop that is implemented in Leica receivers is still based on a standard correlator ("standard" and "corrected" L1 phase are output in the standard and PMMW correlators respectively, both running in parallel). Thus, standard phase measurements are output. The new PMMW correlator has been implemented in parallel to output its own measurements, the purpose of which is to provide a correction of the standard L1 phase measurements. The PMMW output can be considered as an additional observable.

In the presence of multipath, the phase error is given by Eq. 7.1:

$\varphi=\arctan \left(\left[\Sigma_{\mathrm{i}} \alpha_{\mathrm{i}}^{\prime *} \sin \left(\Theta_{\mathrm{i}}\right)\right] /\left[1+\Sigma_{\mathrm{i}} \alpha_{\mathrm{i}}^{\prime *} \cos \left(\Theta_{\mathrm{i}}\right)\right]\right)$

for a number $n$ of reflected signals (from $i=1$ to $i=n$ ), where:

$\alpha_{i}^{\prime}=\alpha_{i}^{*} R\left(d_{i}+\tau\right) / R(\tau)$ 
$\mathrm{R}$ is the code autocorrelation function

$d_{i}$ is the code delay of the $i^{\text {th }}$ reflected signal

$\Theta_{i}$ is the phase shift of the $i^{\text {th }}$ reflected signal

$\tau$ is the multipath code error

$\alpha_{\mathrm{i}}$ is the ratio of amplitude between $\mathrm{i}^{\text {th }}$ reflected signal and the direct signal.

This relation, where the waveform of the reflected signals is preserved, makes the assumption that the reflections follow the law of the geometric optics, which is classically accepted [GEORGIADOU and KLEUSBERG, 1987], [COMP and AXELRAD, 1996] or [RAY et al., 1999].

In the Leica code tracking loop, the multipath code error $\tau$ is bounded, as well as the code delay d of the reflected signals that cause this code error. The bounds are displayed in Fig. 7.1, for the MMW correlator effectively implemented in the receivers used in the campaign of tests (see Chapter $5, \S 5.1 .3$ ). Several multipath code error envelopes are represented, for different values of the ratio of amplitude $\alpha$.

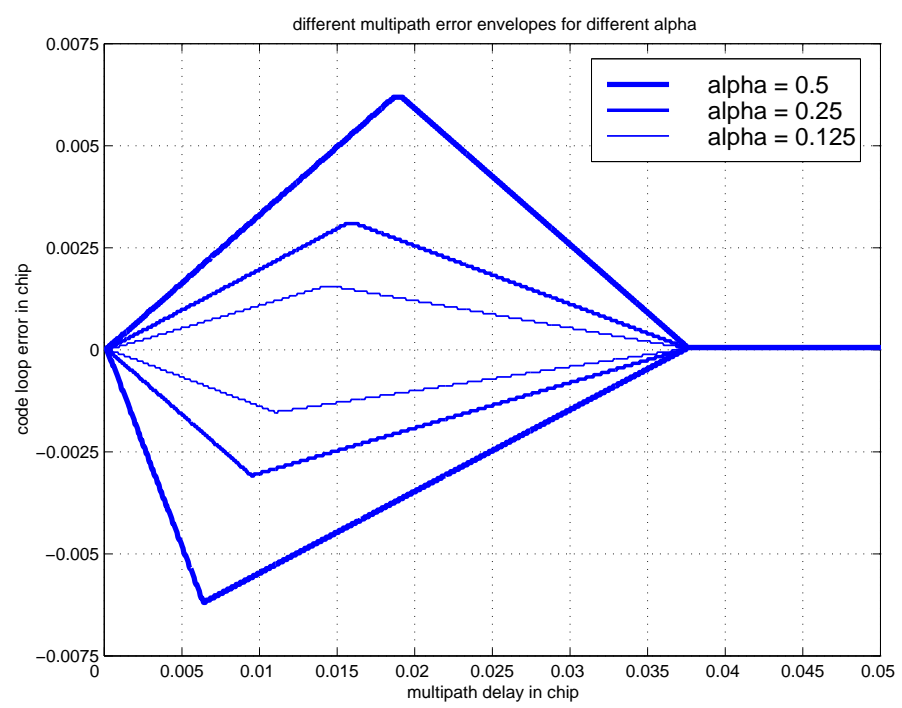

Figure 7.1: code MMW error envelopes for different ratio of amplitude 
The multipath code error $\tau$ does not exceed a few metres. Typically, for the ratio of amplitude identified in Chapter 6 ( $\alpha \sim 0.1$ to $\alpha \sim 0.3$ for the lightweight AT502 antenna used in the experiment), the maximum multipath code error was in the order of 0.0025 chip, or $0.75 \mathrm{~m}$. Moreover, the additional path length $\mathrm{L}$ during the experiment was of the order of a few metres. So $\mathrm{d}$ and $\tau$ are small with respect to the duration of the chip. Consequently, $\alpha^{\prime} \sim \alpha$, and:

$\varphi=\arctan \left(\left[\Sigma_{\mathrm{i}} \alpha_{\mathrm{i}}{ }^{*} \sin \left(\Theta_{\mathrm{i}}\right)\right] /\left[1+\Sigma_{\mathrm{i}} \alpha_{\mathrm{i}}{ }^{*} \cos \left(\Theta_{\mathrm{i}}\right)\right]\right)$

Lastly, the hypothesis that the ratio of amplitude is small permits further simplification. Finally.

$\varphi \sim \sum_{\mathrm{i}} \alpha_{\mathrm{i}} \sin \Theta_{\mathrm{i}}$

\subsection{2 - Code error}

As far as the code measurements are concerned, the theory is based on the code tracking fundamental equation: $\mathrm{DF}=0$, where $\mathrm{DF}$ is the discrimination function implemented in the code correlator. Chapter 4 underlined that the code error envelope of the code MMW correlator is superimposed onto those of the early-late wide and narrow correlators for small code delay $\mathrm{d}$, and that it gets back to zero as soon as d exceeds half the MMW size (i.e. 0.0375 chip for the current implementation, or $11 \mathrm{~m}$ ).

The equation $\mathrm{DF}=0$ (null discrimination function) of the early-late wide and narrow correlators in the presence of multipath is given by Eq. 7.4:

$\cos (\varphi)[R(\tau+\mathrm{p} / 2)-\mathrm{R}(\tau-\mathrm{p} / 2)]+\Sigma_{\mathrm{i}} \alpha_{\mathrm{i}} \cos \left(\varphi-\Theta_{\mathrm{i}}\right) *\left[\mathrm{R}\left(\mathrm{d}_{\mathrm{i}}+\tau+\mathrm{p} / 2\right)-\mathrm{R}\left(\mathrm{d}_{\mathrm{i}}+\tau-\mathrm{p} / 2\right)\right]=0$

for a number $n$ of reflected signals (from $i=1$ to $i=n$ ), where:

$\mathrm{R}$ is the code autocorrelation function

$\mathrm{d}_{\mathrm{i}}$ is the code delay of the $\mathrm{i}^{\text {th }}$ reflected signal 
$\Theta_{i}$ is the phase shift of the $i^{\text {th }}$ reflected signal

$\varphi$ is the multipath phase error

$\tau$ is the multipath code error

$\alpha_{\mathrm{i}}$ is the ratio of amplitude between $\mathrm{i}^{\text {th }}$ reflected signal and the direct signal

$p$ is the chip spacing of the correlator $(p=1$ chip for a wide correlator, $p=0.1$ chip for the

$10 \%$ narrow correlator, and $\mathrm{p}=0.025$ chip for the code MMW equivalent $2.5 \%$ narrow correlator).

No further simplified expression of the equation $\mathrm{DF}=0$ can be derived, because the autocorrelation function, displayed in Fig. 7.2, is only defined at intervals:

- for $-\mathrm{T}<\tau<\mathrm{T}, \mathrm{R}(\tau)=1-|\tau| / \mathrm{T}$;

- for $\tau<-\mathrm{T}$ or $\tau>\mathrm{T}, \mathrm{R}(\tau)=0$;

where $\mathrm{T}$ is the duration of the chip.

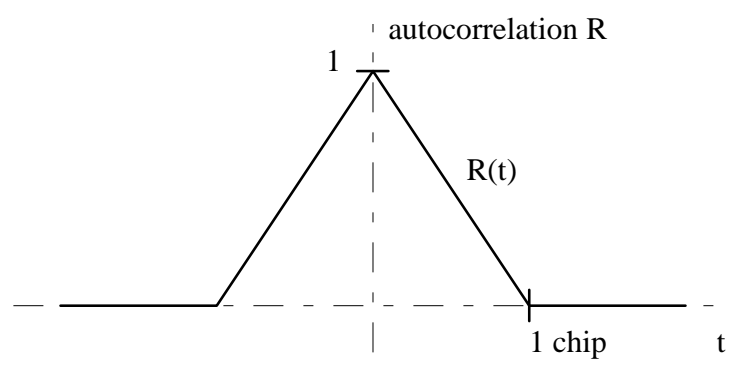

Figure 7.2: the PRN code autocorrelation function in the interval [ \pm 1 chip]

Similarly, the difference $[R(\tau+p / 2)-R(\tau-p / 2)]$ that appears in early-late DFs is also defined at intervals. It is interesting to notice that every DF used in receivers (see Chapter 4, Fig. 4.6), including that used in the code MMW correlator (see Chapter 5, Fig. 5.4, 5.7 and 5.10), has the same linear part around zero, as displayed in Fig. 7.3, whatever the chip spacing $\mathrm{p}$ or the MMW size are. Only the size of this central linear part changes along with the correlator, not its slope. Note that this is why the different code error envelopes are superimposed near zero. 


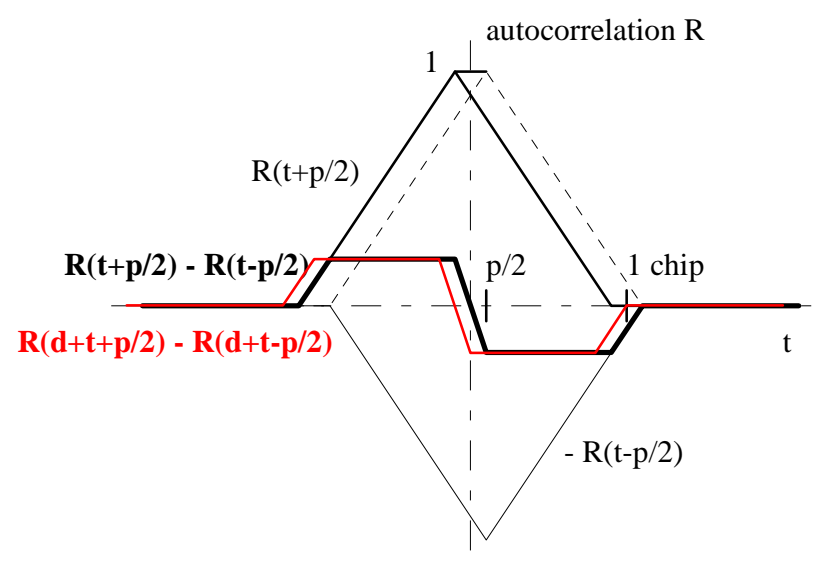

Figure 7.3: the discrimination function of the narrow correlator in the interval [ \pm 1 chip]

A new analysis and simplification of the problem in order to model the multipath code error is given below. The differences $[R(\tau+p / 2)-R(\tau-p / 2)]$ and $\left[R\left(d_{i}+\tau+p / 2\right)-R\left(d_{i}+\tau-p / 2\right)\right]$ that compose the DFs in the presence of multipath have central linear parts. For small code delay d, the central linear parts of the DFs of the direct and reflected signals intersect, which enables these differences to be computed as follows:

$\mathrm{R}(\tau+\mathrm{p} / 2)-\mathrm{R}(\tau-\mathrm{p} / 2)=-2 \tau / \mathrm{T}$

$\mathrm{R}(\mathrm{d}+\tau+\mathrm{p} / 2)-\mathrm{R}(\mathrm{d}+\tau-\mathrm{p} / 2)=-2(\tau+\mathrm{d}) / \mathrm{T}$

and combined in order to derive an expression of the code multipath error:

$\cos (\varphi)[\tau]+\sum_{\mathrm{i}} \alpha_{\mathrm{i}} \cos \left(\varphi-\Theta_{\mathrm{i}}\right) *\left[\tau+\mathrm{d}_{\mathrm{i}}\right]=0$

$\tau=\left(\sum_{\mathrm{i}} \alpha_{\mathrm{i}} \cos \left(\varphi-\Theta_{\mathrm{i}}\right) * \mathrm{~d}_{\mathrm{i}}\right) /\left(\cos (\varphi)+\sum_{\mathrm{i}} \alpha_{\mathrm{i}} \cos \left(\varphi-\Theta_{\mathrm{i}}\right)\right)$

The assumption of a small code delay needs to be precised here. Eq. 7.5a and 7.5b suppose that the multipath code error $\tau$ is included in the intersection of the intervals $[-p / 2$ $\mathrm{p} / 2]$ and [d-p/2 d+p/2]. The code delay $d$ is conventionally positive (see Chapter 4 , $\S 4.1$ ). This intersection exists if $\mathrm{p} / 2>\mathrm{d}-\mathrm{p} / 2$, i.e. if $\mathrm{d}<\mathrm{p}$ (hypothesis $\mathrm{n}^{\circ} 1$ ). 
This intersection is $[\max (-\mathrm{p} / 2, \mathrm{~d}-\mathrm{p} / 2) \min (\mathrm{p} / 2, \mathrm{~d}+\mathrm{p} / 2)]$, i.e. $[\mathrm{d}-\mathrm{p} / 2 \mathrm{p} / 2]$. So it is supposed that $\tau$ is included in the intervals [d-p/2 p/2] (hypotheses $n^{\circ} 2$ and $n^{\circ} 3$ ).

It is convenient to understand graphically the meaning of the hypotheses made above. Fig. 7.4 is based on Fig. 7.1. The early-late narrow correlator equivalent to the implemented code MMW correlator is the $2.5 \%$ narrow correlator, for which $\mathrm{p}=0.025$ chip. Its envelope is superimposed in red on Fig. 7.4, for $\alpha=0.5$. The red dashdot line corresponds to the limit case when $\alpha=1$.

1st hypothesis: $d<$ p, i.e. $d<0.025$ chip, is represented by a first straight $(\mathrm{H1})$; 2nd hypothesis: $d-p / 2<\tau$, i.e. $\tau>d-0.0125$ chip, is represented by a second straight $(\mathrm{H} 2)$; 3rd hypothesis: $\tau<$ p/2, i.e. $\tau<0.0125$ chip, is represented by a third straight $(\mathrm{H} 3)$.

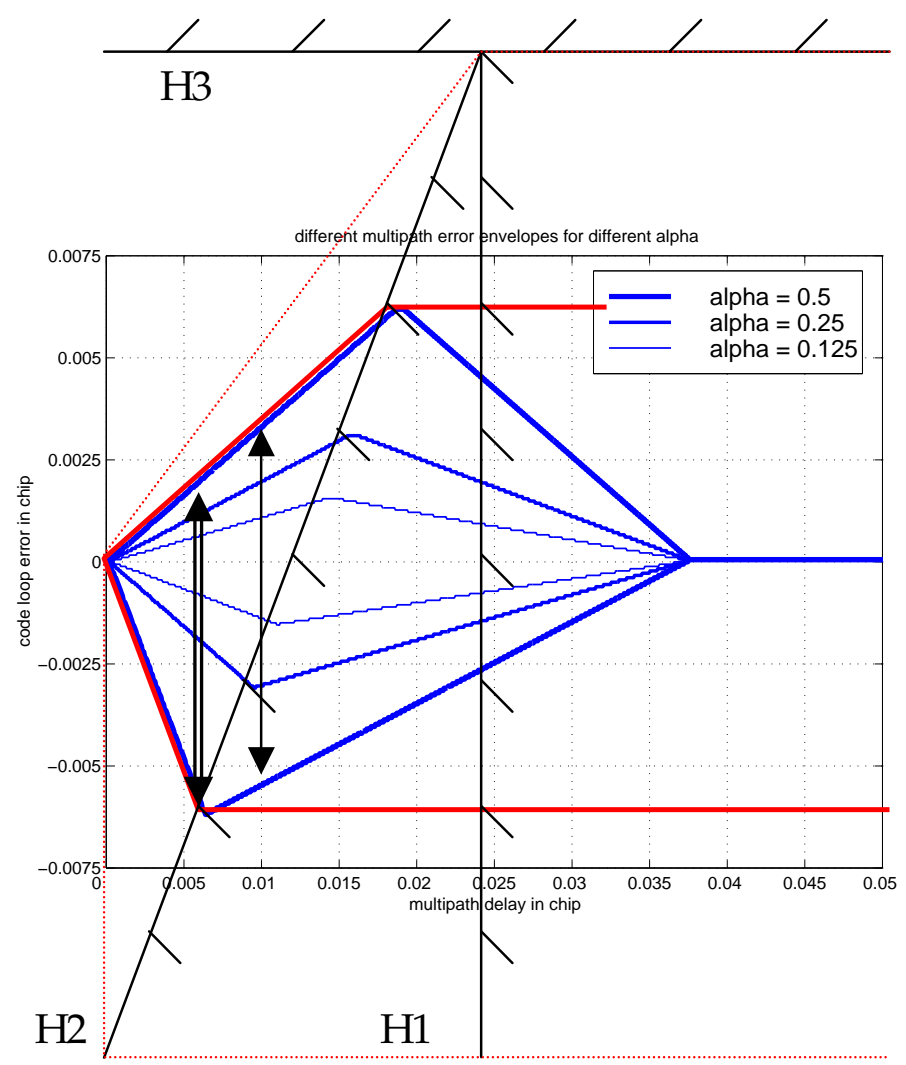

Figure 7.4: multipath code error envelopes for different ratios of amplitude corresponding to the implemented code MMW correlator 
On the example of a delay of 0.01 chip, one can obviously see that part of the possible multipath code error for $\alpha=0.5$ (shown by the simple arrow) is under the straight $\mathrm{H} 2$, in which the equations $7.5 \mathrm{a}$ and $7.5 \mathrm{~b}$ are not valid.

For $\alpha=0.5$ again, but for a code delay $\mathrm{d}$ of only 0.00625 chip, the entire possible multipath code error (shown by the double arrow) is above the straight $\mathrm{H} 2$. Then the aforementioned equations are effectively valid.

So, for a given ratio of amplitude $\alpha$, there is a threshold of the code delay $\mathrm{d}$ under which any multipath code error will be given by Eq. 7.7. Note that this threshold is 0.00625 chip, for $\alpha=0.5$. It depends on the ratio of amplitude $\alpha$.

In the presence of multipath with a ratio of amplitude like that estimated during the experiment ( $\alpha \sim 0.1$ to $\alpha \sim 0.3$ ), the domain of validity of Eq. 7.7 corresponds to a threshold of approximately 0.01 chip, or $3 \mathrm{~m}$.

Under this threshold of code delay (or additional path length), another approximation of Eq. 7.7 consists in neglecting the multipath phase error $\varphi$ :

$\tau \sim\left(\sum_{\mathrm{i}} \alpha_{\mathrm{i}} \cos \left(\Theta_{\mathrm{i}}\right) * \mathrm{~d}_{\mathrm{i}}\right) /\left(1+\sum_{\mathrm{i}} \alpha_{\mathrm{i}} \cos \left(\Theta_{\mathrm{i}}\right)\right)$

Lastly, with the additional hypothesis of a small ratio of amplitude, the code error is:

$\tau \sim \sum_{\mathrm{i}} \alpha_{\mathrm{i}} \cos \Theta_{\mathrm{i}}^{*} \mathrm{~d}_{\mathrm{i}}$

which means that the upper and lower envelopes of the multipath code error are considered approximately symmetric, whereas their actual slopes are respectively $\alpha_{\mathrm{i}} /(1+$ $\left.\alpha_{i}\right)$ and $-\alpha_{i} /\left(1-\alpha_{i}\right)$.

\subsection{3 - SNR (or C/N0) variation}

Multipath has also effects on the signal-to-noise ratio or C/N0 (see Chapter 4, § 4.3). 
Eq. 7.10 describes the power of the received signal, made of one direct component and one or several reflected components:

$\mathrm{P}=\mathrm{P}_{\text {direct }}{ }^{*} \mathrm{R}^{2}(\tau)^{*}\left(1+2 \Sigma_{\mathrm{i}} \alpha_{\mathrm{i}}^{\prime} \cos \left(\Theta_{\mathrm{i}}\right)\right)$

for a number $n$ of reflected signals (from $i=1$ to $i=n$ ), where $P_{\text {direct }}$ is the power of the direct signal, which is usually given in $\mathrm{dB}: \mathrm{P}^{\mathrm{dB}}=10 \log _{10}(\mathrm{P})$.

Consider again that $\alpha^{\prime} \sim \alpha$ (which supposes that the code delay and the multipath code error are small) and that $2 \Sigma_{i} \alpha_{i} \cos \left(\Theta_{i}\right)<<1$, i.e. that the ratio of amplitude is small. The logarithm can be approximated by its first order Taylor's expansion.

Practically the receiver outputs an estimation of the signal-to-noise ratio, denoted SNR (or $\mathrm{C} / \mathrm{N} 0$, after normalising by the bandwidth of the tracking loop). Hence, the power of the received signal is not available directly. However, the above equation remains valid for the signal-to-noise ratio, since $\mathrm{P}^{\mathrm{dB}}$ and the signal-to-noise ratio differ only by an additive term corresponding to the power of the noise.

The variation of the power of the received signal (as well as that of SNR or C/N0) around its nominal (direct) value in $\mathrm{dB}$ is given by.

$\mathrm{SNR}_{\text {multipath }} \sim \mathrm{K} \sum_{\mathrm{i}} \alpha_{\mathrm{i}} \cos \Theta_{\mathrm{i}}$

where $K$ is a constant ( $K=20 / \ln (10))$, independent of the index $i$ of the direct and reflected signals; $\mathrm{K}$ differs from a GPS receiver to another, because the manufacturers do not necessarily implement the same formula to output SNR (or C/N0).

For the GPS user, this means practically that a calibration of the signal-to-noise ratio is necessary first, in a multipath free environment, from which a template function (signal-tonoise ratio versus satellite elevation) can be derived. 


\section{2 - The multipath observables}

The multipath observables are:

- the variation of the signal-to-noise ratio, with respect to a satellite elevation template function;

- the code error (only for a baseline up to a few kilometres), obtained by computing the variation around the average value of the ionospheric L1 and L2 combination (see Chapter 2, § 2.3.1.1): C1 - (1+2/((f1/f2 $\left.\left.)^{2}-1\right)\right) \Phi 1+\left(2 /\left((\mathrm{f} 1 / \mathrm{f} 2)^{2}-1\right)\right) \Phi 2$;

- the PMMW correction, that (contrary to the signal-to-noise ratio) deteriorates when the panel gets close.

Fig. 7.5 illustrates the observables listed here. The three consecutive days of the second campaign of tests for SV2 are displayed: they correspond to 1, 4 and $7 \mathrm{~m}$ distance.

Signal-to-noise ratio, code error, PMMW and additionally, the Observed - Computed Double Difference of L1 phase are superimposed. The quadrature between the phase multipath error on the one hand, and the code and signal-to-noise ratio on the other hand are clearly visible, except for the test corresponding to the reflector at only $1 \mathrm{~m}$ (Fig. ${ }^{\circ} .5 \mathrm{a}$ ).

Fig. 7.6 gives a few geometrical characteristics of the multipath. The five frames in each of the three parts contain the following information:

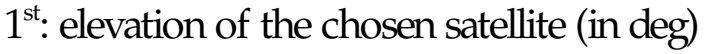

$2^{\text {nd }}$ : azimuth of the chosen satellite (in deg)

$3^{\text {rd }}$ : additional path length of the reflected signal (in $\mathrm{m}$ )

$4^{\text {th }}$ : elevation of the reflected ray (in deg) with respect to the plane of the antenna

$5^{\text {th: }}$ "track" of the point of reflection in the plane of the panel (underlined in green).

Except for the fifth diagram, all diagrams are time scaled so that they refer exactly to the period in which the panel causes multipath on the signal from SV2. 


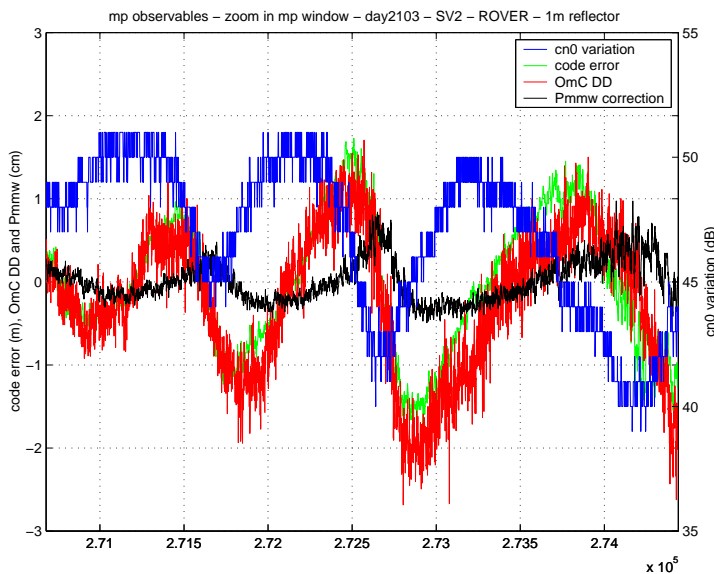

Figure 7.5a: observables and O-C L1 DD (1 m)

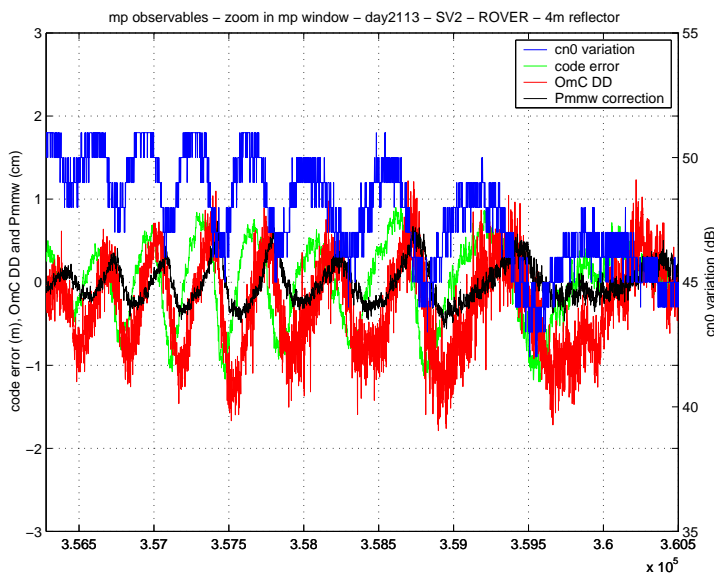

Figure 7.5b: observables and O-C L1 DD (4 m)

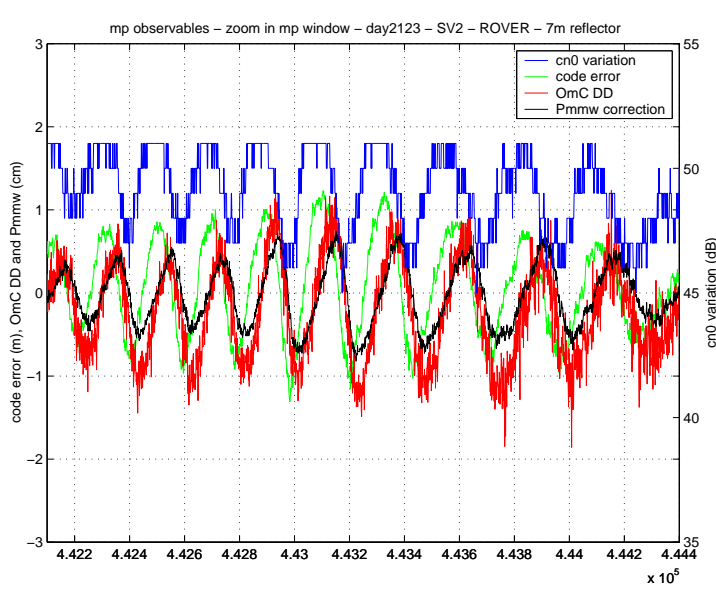

Figure 7.5c: observables and O-C L1 DD (7 m)

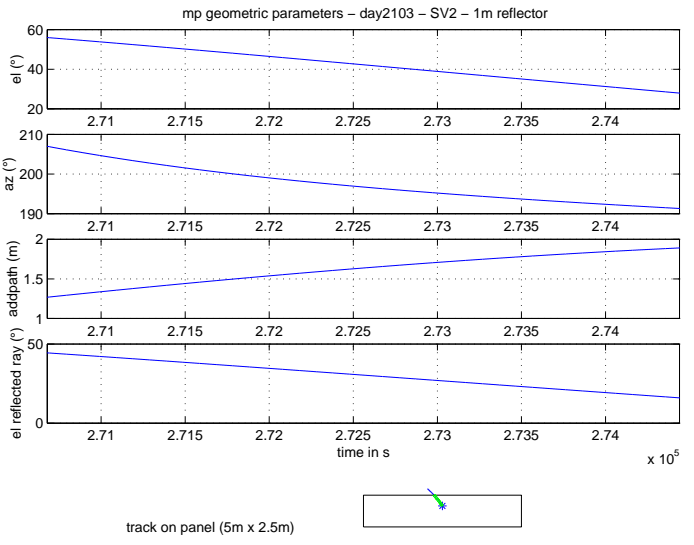

Figure 7.6a: geometrical characteristics $(1 \mathrm{~m})$

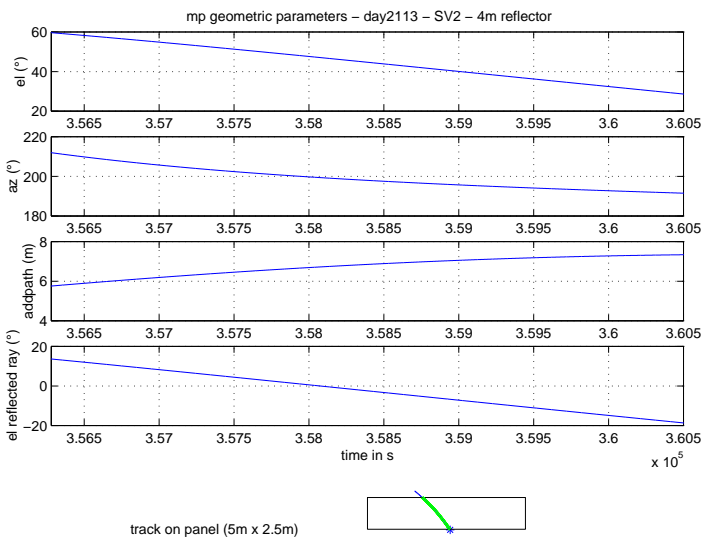

Figure 7.6b: geometrical characteristics $(4 \mathrm{~m})$

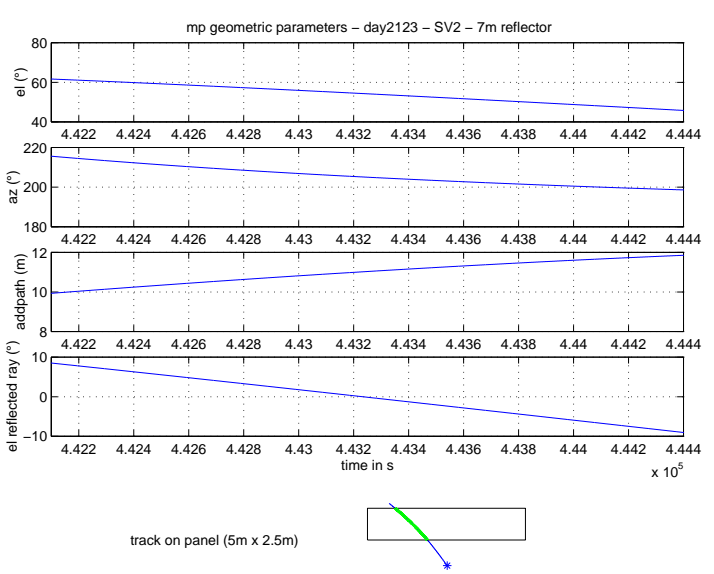

Figure 7.6c: geometrical characteristics $(7 \mathrm{~m})$

These observables are here displayed for a specific satellite, but the patterns are the same for any other, as well as the relationship between. 


\section{3 - The multipath phase error reconstruction}

7.3.1 - SNR (or C/N0) based multipath phase error reconstruction

\subsubsection{1 - introduction}

The SNR-based multipath phase error correction was first introduced by [COMP and AXELRAD, 1996]. This method is based on the fact that the signal-to-noise ratio varies harmonically around its nominal value in the presence of multipath (see Eq. 7.11).

The nominal value of SNR can be determined from a preliminary set of data, by fitting a polynomial to SNR in function of the elevation. Of course, such data must be collected in a clean environment, and the obtained template function depends on the antenna, the receiver and the cable between them. This polynomial fitting was processed on the data collected with no panel during day209 (see Chapter 6, table 6.1).

It appears in Eq.7.11 that the multipath variation of the signal-to-noise ratio $\left(\mathrm{SNR}_{\text {multipath }}\right.$ or $\left.\mathrm{C} / \mathrm{NO}_{\text {multipath }}\right)$ is a sum of sinusoidal components.

An identification of the amplitudes (denoted $\hat{\mathrm{A}}_{\mathrm{i}}$ ) and the arguments (denoted $\hat{\mathrm{h}}_{\mathrm{i}}$ ) of these components is possible by combining different classical algorithms of signal processing (firstly an "Adaptive Notch Filter" for frequency and amplitude identification, and secondly an "Adaptive Least Squares" for amplitude and argument identification). A comprehensive description of these algorithms can be found in [NEHORAI, 1985] and [HANDEL and TICHAVSKY, 1994].

The combination of ANF and ALS has already been investigated and applied on GPS signal-to-noise ratio by [COMP and AXELRAD, 1996] and [BARNES, 2000]. It can be applied to any signal that shows one or several sine waves (i.e. one or several peaks in the Fourier transform). [COMP, 1996] shows the benefit of combining the two different signal processes (ANF and ALS) in order to identify, in the signal-to-noise ratio, the time-varying sinusoidal components due to multipath. The two steps contained in the combined ANF\&ALS process are summarised in § 7.3.1.2 and § 7.3.1.3. 


\subsubsection{2 - Adaptive Notch Filter}

The first step, ANF, was originally designed [NEHORAI, 1985] in order to identify and remove multiple non-stationary sine waves embedded in a signal (non-stationary means that the frequency and the amplitude of the sine waves vary with time). To do so, the algorithm requires a priori the number of sine waves to be identified and removed, and two coefficients: a parameter of convergence, and a pole-zero contraction factor. These are respectively related to the quality (accuracy) of the estimated frequencies, and the ability of the algorithm to track these frequencies in a dynamic process.

The input signal $S$ must be sampled at a frequency in accordance with the sine waves that are to be filtered. The Nyquist frequency applies, but it is recommended that the sampling frequency be around ten times the maximum expected frequency.

The outputs are the time varying frequencies $\left(\hat{\mathrm{w}}_{\mathrm{i}}\right)$ and secondarily the amplitudes and the phases, that are unused in case of processing ALS just after.

\subsubsection{3 - Adaptive Least Square}

The second step of the combined ANF\&ALS process, ALS, takes the advantage of the first step. ALS actually determines, for each embedded sine wave with a priori known frequency $\left(\hat{\mathrm{w}}_{\mathrm{i}}\right)$ given by ANF, the corresponding amplitudes and phases. The same signal $\mathrm{S}$ is input, as well as the a priori identified frequencies. Once the frequencies are known, it can be shown that the problem is linear with respect to the amplitudes and the phases [HANDEL and TICHAVSKY, 1994]. The parameters that govern the process are again a parameter of convergence, related to the quality (accuracy) of the estimated amplitudes and phases, and also a forgetting factor for dynamic ability.

The outputs are the time varying amplitudes $\left(\hat{\mathrm{A}}_{\mathrm{i}}\right)$ and phases (or arguments $\hat{\mathrm{h}}_{\mathrm{i}}$, which are the phases modulo $\left.2 \pi \hat{\mathrm{w}}_{\mathrm{i}} \mathrm{t}\right)$, such that the input signal $\mathrm{S}$ equals:

$$
S=\sum_{\mathrm{i}} \hat{A}_{\mathrm{i}} \sin \hat{\mathrm{h}}_{\mathrm{i}}
$$




\subsubsection{4 - Application of the combined ANF\&ALS process to SNR}

The combined ANF\&ALS process enables the identification of varying amplitudes and varying arguments from the multipath variation of the signal-to-noise ratio for an a priori unlimited number of reflections. The outputs $\left(\hat{\mathrm{A}}_{\mathrm{iSNN}}\right.$ and $\left.\hat{\mathrm{h}}_{\mathrm{iSNR}}\right)$ are such that:

$\mathrm{SNR}_{\text {multipath }}=\sum_{\mathrm{i}} \hat{\mathrm{A}}_{\mathrm{iSNR}} \sinh \hat{\mathrm{h}}_{\mathrm{iSNR}}$

For static multipath, the sampling frequency is fixed at $0.05 \mathrm{~Hz}$ (1 data, out of 20 , is input in the identification process), which is enough since the observed multipath have standard periods of a more than 4 minutes (see Chapter 6, Fig. 6.13c to 6.15c). The same process was applied to data logged at both base and rover, and for 1 main reflection $(i=1)$.

This identification provides a way to build a phase correction. Actually, the multipath phase error given in Eq. 7.3 shows exactly the same amplitude $\left(\alpha_{i}\right)$ and argument $\left(\Theta_{i}\right)$ as the multipath variation of the signal-to-noise ratio.

Hence, $\hat{\mathrm{A}}_{\mathrm{i} S \mathrm{NR}}$ and $\hat{\mathrm{h}}_{\mathrm{i} S \mathrm{NNR}}$ identified before from the SNR variation can be introduced in Eq. 7.3 (with $\alpha_{\mathrm{i}}=\hat{\mathrm{A}}_{\mathrm{iSNR}} / \mathrm{K}$ and $\Theta_{\mathrm{i}}=\pi / 2-\hat{\mathrm{h}}_{\mathrm{iSNR}}$ ), to carry out the phase correction:

$$
\varphi=1 / \mathrm{K} \sum_{\mathrm{i}} \pm \hat{\mathrm{A}}_{\mathrm{iSNR}} \sin \left(\pi / 2-\hat{\mathrm{h}}_{\mathrm{i} \backslash \mathrm{SNR}}\right)
$$

Nevertheless, the identification from the variation of SNR or C/N0 does not give the sign of the argument, since SNR or C/N0 only enables the recovery of the sine of this argument, and not the cosine, therefore the sign remains undetermined. Consequently, the sign of the phase correction cannot be identified by this way.

The sign ambiguity has a physical interpretation. The reflected signal travels an additional path length, to which the delay $\mathrm{d}$ is proportional. While this additional path length varies, the phase shift $\Theta$ of the reflected signal (relative to the direct) rotates. The sense of the rotation depends on the additional path length increases or decreases. 
Before the additional path length reaches its minimum, the SNR variation (as well as the multipath code error $\tau)$ is, let say, $+90^{\circ}$ offset with respect to the multipath phase error $\varphi$.

After the additional path length reaches its minimum, the SNR variation (as well as the multipath code error $\tau$ ) is, in accordance to before, $-90^{\circ}$ offset with respect to the multipath phase error $\varphi$.

With no a priori idea of the relative position of the rover, the satellite and the reflector in the environment, the sign of the phase shift is unknown. The variation of the additional path length (i.e. whether it increases or decreases) is indeed not known a priori. And neither the information of the SNR variation nor the code error can solve this ambiguity.

In the particular case of the tests performed in the frame of this research, the unknown sign can be determined by fitting the reconstructed phase error and the Observed-Computed DD of L1 phase, computed on known points.

In the general case of unknown points, the DD residuals should be used. The choice of the sign must be done to fit these residuals as well as possible.

In real-time processing, such determination requires the analysis of residuals in a moving window, with a size that needs to be ascertained by tuning. This process induces a time shift, or latency, that will impact on the ability of the method to be truly real-time. Also, if the environment changes rapidly, incorrect signs may result due to the fact that residuals also absorb part of the multipath error, especially if there are only a few satellites (and hence limited redundancy), which is often the case in typical multipath environments.

One of the objectives of this thesis is to address this drawback of the phase error reconstruction based on the signal-to-noise ratio, and suggest an original way to determine the sign of the phase correction by using the phase MMW estimate of the phase error. 


\subsection{2 - SNR (or C/N0) and phase MMW based reconstruction}

The investigation reported in this section is based on the fact that the phase MMW provides an estimate of the multipath phase error $\varphi$, including its sign.

Moreover, a key observation is that the phase MMW measurement is not completely null when the reflector is close, but actually attenuated only, as shown in Fig 7.5. In this figure, the phase MMW measurement becomes weak compared to the expected multipath phase error when the distance to the panel decreases. On the contrary, when the reflector is sufficiently far, then the phase MMW measurement fits the effective phase error well and enables an effective correction of the standard phase.

The idea is to take advantage of the information carried out by the phase MMW process, even if the reflector gets close by the antenna.

This section investigates the interest of using the phase MMW for the direct identification of the frequencies and phases (arguments) in the multipath error.

Note that the identified amplitudes in the variation of the signal-to-noise ratio remain necessary to fix properly the amplitudes in the reconstructed phase correction. Those (contrary to the phase MMW measurement) actually do not deteriorate when the reflector gets close.

Different possibilities are tested, depending on the ANF step of the frequencies identification process:

-1st: as in §7.3.1, the first possible use of the phase MMW consists of the identification of the frequencies in this signal at the ANF step. Then, the phase MMW-based identified frequencies (denoted wi $\backslash$ PMMW) are fed into the ALS step of the process, in which the variation of the signal-to-noise ratio is input. The amplitudes and the phases are thus estimated. The last step is unchanged: it is the reconstruction of the multipath phase error $\varphi$ given by Eq. 7.14. Note that the problem of sign ambiguity remains in this scenario: consequently, it will not be tested further. 
- $2^{\text {nd: }}$ using not only the frequencies, but also the phases of the MMW measurement, the use of this observable is extended, in order to limit that of SNR to the estimation of amplitudes only. Like in the $1^{\text {st }}$ scenario, the frequencies $\hat{\mathrm{w}}_{\mathrm{i} \backslash \mathrm{PMMW}}$ are identified from the phase MMW measurement. Afterward, two ALS parallel steps are executed. On the one hand, the phase MMW-based ALS provides with the phases (amplitudes are unused). On the other hand, the SNR-based similar identification outputs the amplitudes (as well as phases, unused). ALS outputs are crossed in the reconstruction of the phase error: it is built with the amplitudes output from the SNR-based process and the arguments output from the MMW-based process.

- $3^{\text {rd: }}$ the same as the $2^{\text {nd }}$ scenario, but the frequencies are identified from the SNR measurement, which gives $\hat{\mathrm{w}}_{\mathrm{i} \backslash \mathrm{SNR}}$ similarly as in $\S 7.3 .1 .4$, and fed to both ALS steps.

- $4^{\text {th }}$ : each ALS step uses the frequencies ( $\hat{\mathrm{w}}_{\mathrm{i} \backslash \mathrm{SNR}}$ and $\left.\hat{\mathrm{w}}_{\mathrm{i} \backslash \mathrm{PMMN}}\right)$ output from its own ANF step.

The basic ideas of each algorithm are summarised in Fig. 7.7.

$\underline{\text { SNR only based process }}$

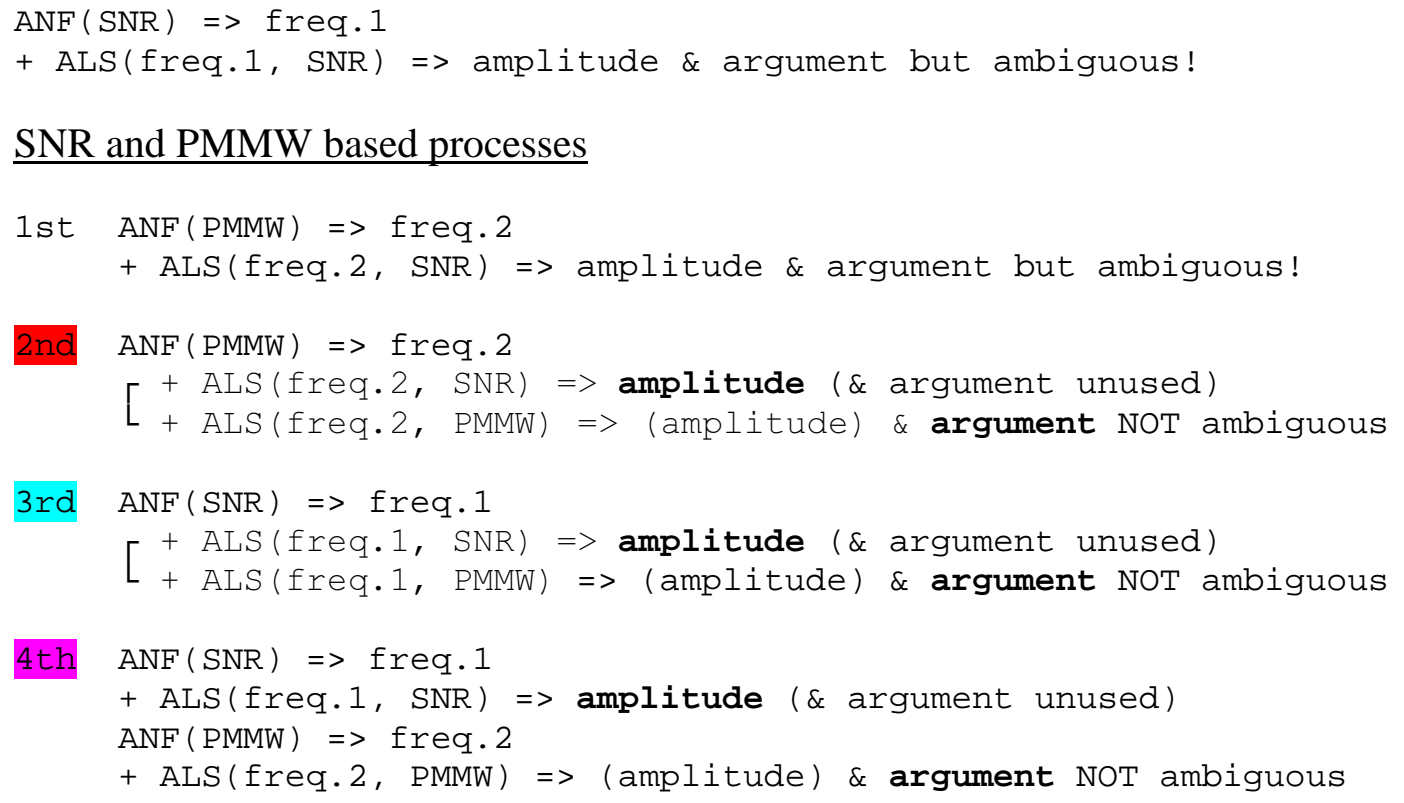

Figure 7.7: different SNR and PMMW mixed scenarios 
In the last 3 scenarios, the ALS outputs (phases $\hat{\mathrm{h}}_{\mathrm{i} \text { PMMN }}$ from PMMW and amplitudes $\hat{\mathrm{A}}_{\mathrm{iSNR}}$ from SNR) are mixed in the following equation, with no sign ambiguity since the sine waves embedded in the phase MMW measurement are the same as those in the multipath phase error that is reconstructed.

$\varphi=1 / \mathrm{K} \sum_{\mathrm{i}} \hat{\mathrm{A}}_{\mathrm{i} \backslash \mathrm{SNR}} \sin \left(\hat{\mathrm{h}}_{\mathrm{i} \backslash \mathrm{PMMW}}\right)$

Contrary to the case of identification of the phases from the variation of the signal-tonoise ratio, this method identifies them directly from the phase MMW measurements and so suppresses the issue of the ambiguity of sign. When reconstructing the phase correction, the required sign is produced directly, which enables the running of the process more automatically than before, when fitting the L1 DD residuals was necessary.

Fig. 7.8 and 7.9 display the standard deviation of the corrected O-C DD of L1 phase versus a variable gain $\mathrm{k} / \mathrm{K}$, where $\mathrm{k}=0.1,0.2,0.3 \ldots 2.0$ and again $\mathrm{K}=20 / \ln (10)$, applied in the phase error reconstruction processes. In these $\mathrm{DD}$, only the phase data for the selected satellite (SV1 or SV2) are corrected, not the differencing satellite. The "blue $(+)$ " line corresponds to the use of signal-to-noise ratio only in the reconstruction process (see §7.3.1). The required sign has been determined with respect to the O-C DD of L1 phase. The "red (o)", "cyan $(x)^{\prime \prime}$ and "magenta $\left(^{*}\right)$ " lines refer to respectively the $2^{\text {nd }}, 3^{\text {rd }}$ and $4^{\text {th }}$ scenarios listed Fig. 7.7. Again, these mixed reconstruction scenarios need no sign determination, since the reconstruction is based on the argument output from PMMW (and also on the amplitude output from signal-to-noise ratio) and is unambiguous.

Note that these three mixed reconstruction scenarios only differ through the frequencies identification in ANF step and use in ALS step, whether it is obtained from the phase MMW measurements, or the SNR, or both.

The two horizontal lines in Fig. 7.8 and 7.9 represent the standard deviation of the Observed - Computed DD of L1 phase, computed with the initial measurements, and with the PMMW corrected ones. These standard deviations are similar as those included in Chapter 6 , tables 6.3 or 6.4 . 


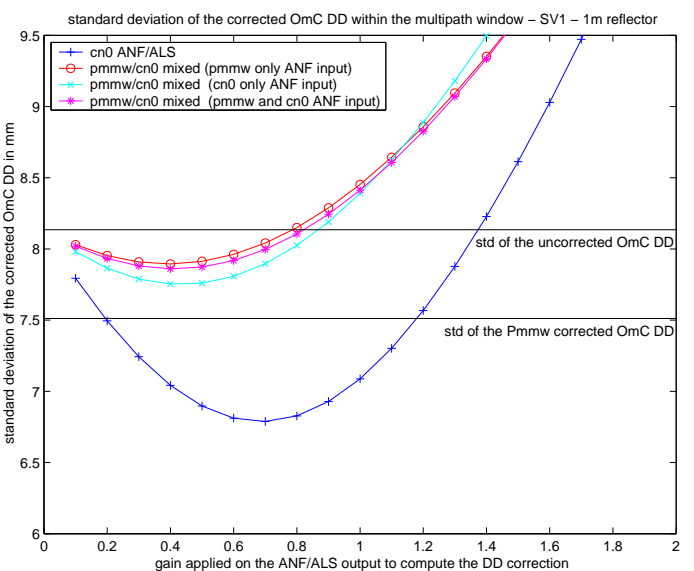

Figure 7.8a: gain tuning for SV1 (1 m test)

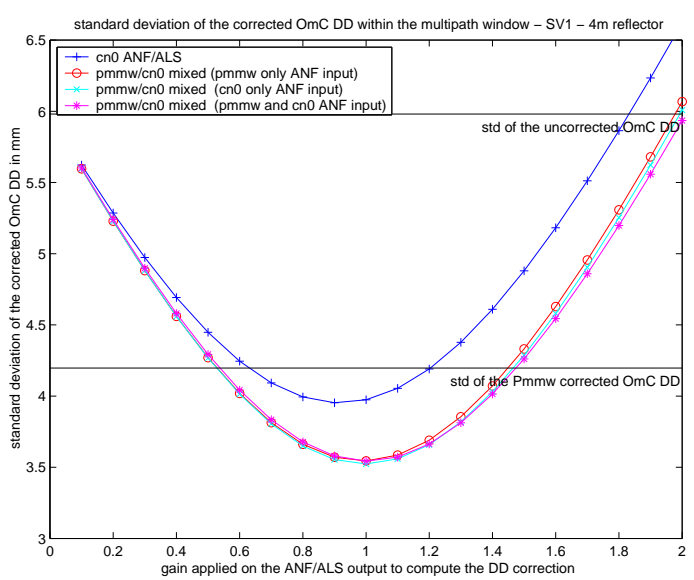

Figure 7.8b: gain tuning for SV1 (4 m test)

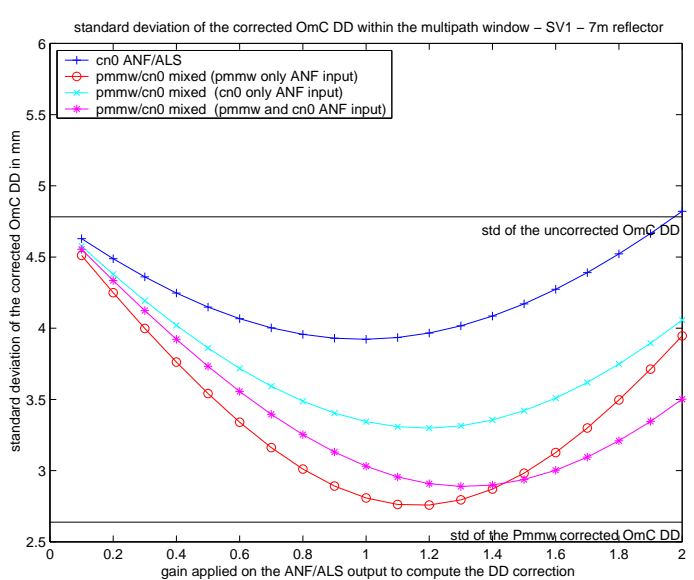

Figure 7.8c: gain tuning for SV1 (7 m test)

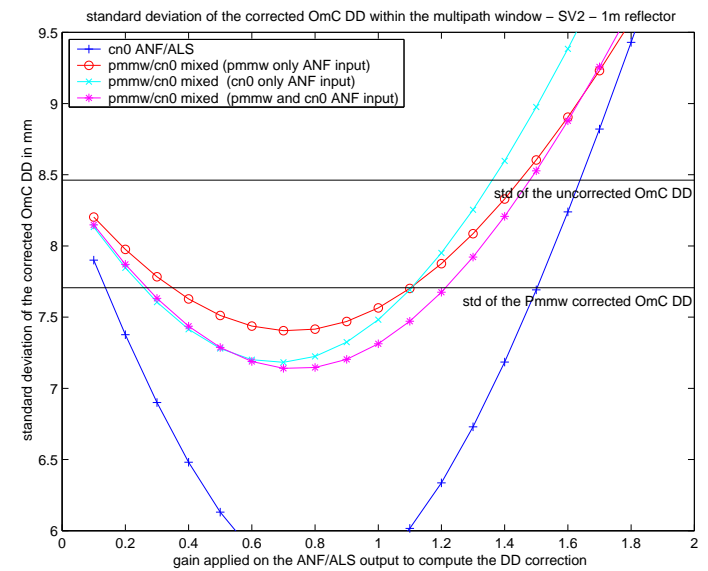

Figure 7.9a: gain tuning for SV2 (1 $\mathrm{m}$ test)

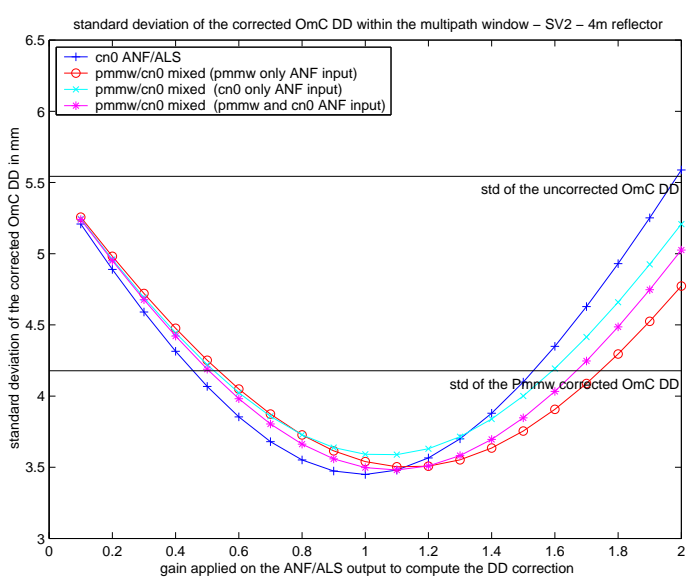

Figure 7.9b: gain tuning for SV2 (4 m test)

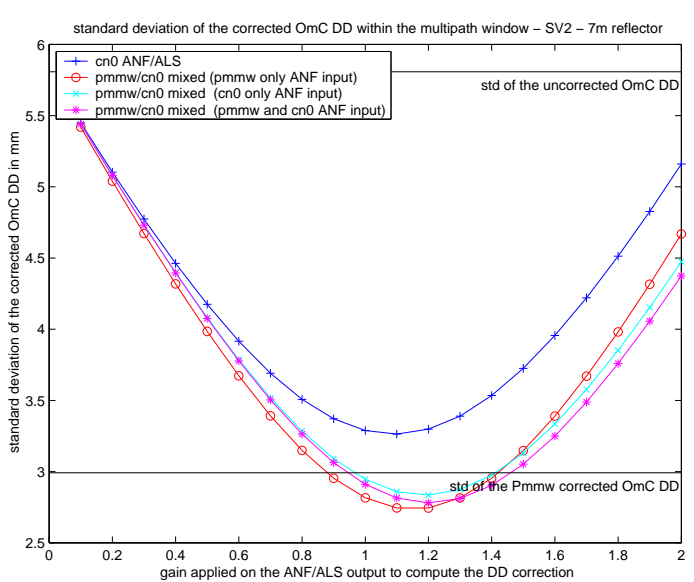

Figure 7.9c: gain tuning for SV2 (7 $\mathrm{m}$ test) 
Table 7.1 contains the minimum standard deviations and the corresponding gain $\mathrm{k}$, for all reconstruction processes and for the selected satellites SV1 and SV2. The stability of this gain (around unity if the theoretical modelling was effective) and the sharpness of the parabolic curve guarantee that the reconstruction is not dependent on the distance to the reflector, nor does it depend on the satellite being considered.

The results (minimum standard deviations of observed-computed L1 phase DD and the corresponding reconstruction gain), for satellites SV1 and SV2, are summarised below. The order is: SNR based only, scenarios 2, 3 and 4 mixed reconstructions.

\begin{tabular}{|c|c|c|c|c|}
\hline $\begin{array}{l}\text { Distance } \\
\text { (to antenna) }\end{array}$ & $\begin{array}{c}\text { SV1 } \\
\text { Std and gain k }\end{array}$ & $\begin{array}{l}\text { Std } \sigma \\
/ \\
\operatorname{Std} \sigma_{\text {PMMW }}\end{array}$ & $\begin{array}{c}\text { SV2 } \\
\text { Std and gain } \mathrm{k}\end{array}$ & $\begin{array}{l}\operatorname{Std} \sigma \\
/ \\
\operatorname{Std} \sigma_{\mathrm{PMMW}}\end{array}$ \\
\hline \multirow[t]{4}{*}{ reflector $1 \mathrm{~m}$} & $6.8 \mathrm{~mm} / 0.7$ & $8.1 \mathrm{~mm}$ & $5.6 \mathrm{~mm} / 0.8$ & $8.5 \mathrm{~mm}$ \\
\hline & $7.9 \mathrm{~mm} / 0.4$ & / & $7.4 \mathrm{~mm} / 0.7$ & / \\
\hline & $7.8 \mathrm{~mm} / 0.4$ & $7.5 \mathrm{~mm}$ & $7.2 \mathrm{~mm} / 0.7$ & $7.7 \mathrm{~mm}$ \\
\hline & $7.9 \mathrm{~mm} / 0.4$ & & $7.1 \mathrm{~mm} / 0.7$ & \\
\hline \multirow[t]{4}{*}{ reflector $4 \mathrm{~m}$} & $4.0 \mathrm{~mm} / 0.9$ & $6.0 \mathrm{~mm}$ & $3.4 \mathrm{~mm} / 1.0$ & $5.5 \mathrm{~mm}$ \\
\hline & $3.5 \mathrm{~mm} / 1.0$ & / & $3.5 \mathrm{~mm} / 1.1$ & / \\
\hline & $3.5 \mathrm{~mm} / 1.0$ & $4.2 \mathrm{~mm}$ & $3.6 \mathrm{~mm} / 1.1$ & $4.2 \mathrm{~mm}$ \\
\hline & $3.5 \mathrm{~mm} / 1.0$ & & $3.5 \mathrm{~mm} / 1.1$ & \\
\hline \multirow[t]{4}{*}{ reflector $7 \mathrm{~m}$} & $3.9 \mathrm{~mm} / 1.0$ & $4.8 \mathrm{~mm}$ & $3.3 \mathrm{~mm} / 1.1$ & $5.8 \mathrm{~mm}$ \\
\hline & $2.8 \mathrm{~mm} / 1.2$ & / & $2.7 \mathrm{~mm} / 1.2$ & / \\
\hline & $3.3 \mathrm{~mm} / 1.2$ & $2.6 \mathrm{~mm}$ & $2.8 \mathrm{~mm} / 1.2$ & $3.0 \mathrm{~mm}$ \\
\hline & $2.9 \mathrm{~mm} / 1.3$ & & $2.8 \mathrm{~mm} / 1.2$ & \\
\hline
\end{tabular}

Table 7.1: optimal standard deviation and corresponding gain for different reconstruction scenarios (for SV1 and SV2) 
The following comments are offered on the results in table 7.1:

- the gain $\mathrm{k}$ is fairly constant (around 1.1) except at $1 \mathrm{~m}$ : this is due to the deterioration of the Phase MMW measurements (they no longer fit the real phase error);

- the three mixed reconstruction scenarios are quasi equivalent, with a slightly less efficient correction for that sharing the SNR based frequencies $\left(3^{\text {rd }}\right.$ scenario, "cyan $\left.(x)^{\prime \prime}\right) \ldots$ but no significant differences between the scenarios can be seen;

- these scenarios, besides the fact that they are unambiguous, provide globally a more efficient or at least equal correction than the classical SNR based reconstruction, except in the case of the very close reflector. With the panel at $7 \mathrm{~m}$, the best results are obtained by applying the PMMW correction directly. At $4 \mathrm{~m}$, the reconstruction processes are equivalent as the PMMW direct correction, even slightly better since the effectiveness of the PMMW starts to deteriorate;

- lastly, at $1 \mathrm{~m}$, the PMMW estimation has deteriorated: so, it seems there is no improvement resulting from a SNR/PMMW mixed reconstruction, especially if the gain is supposed to be fixed to the value obtained before, i.e. 1.1. On the contrary, SNR based process remains potentially the most efficient (provided the problem of sign ambiguity is solved).

The phase MMW reconstruction remains efficient in the case of the reflector at 4 and $7 \mathrm{~m}$, and that it is always better and easier (because it directly gives the correct sign) than when using only the signal-to-noise ratio. Nevertheless, the reconstruction based on the phase MMW fails in the case of the reflector at $1 \mathrm{~m}$.

Although these tests are based on limited data (and more should be carried out before firm conclusions are drawn) they do indicate that the phase MMW measurement probably does not carry sufficient relevant information to compute a significantly improved phase correction in the case of very close reflectors. 


\subsection{3 - Use of code error}

This section concentrates on the multipath code error observable and its potential to indicate phase multipath error. It has been shown that the reconstruction of the multipath phase error from the code error only is theoretically impossible since the amplitude identified from the code error depends not only on $\alpha$ but also on the additional path length (i.e. the code delay d), whereas that of the phase error depends on $\alpha$ only (see Eq. 7.3 and 7.9). There is a trivial problem of observability and the code error is not sufficient to solve the inverse problem of separating the multipath ratio of amplitude $\alpha$ and the code delay $\mathrm{d}$.

However, it seems that the argument of the code error progressively shifts as the additional path diminishes. This is particularly true for the test at $1 \mathrm{~m}$ and it is visible on Fig. 7.5a. The code error, that was in phase with the SNR variation and in quadrature with the phase error for the tests at 4 and $7 \mathrm{~m}$, shifts to become in quadrature with the SNR variation and in phase with the phase error at around $1.5 \mathrm{~m}$ additional path. This is observed for all satellites. Further tests with different receivers might indicate whether or not this is due to details of Leica's implementation (e.g. sampling strategy or pre-correlation bandwidth), and if it is applicable to a certain class of multipath mitigation techniques.

There appears to be great potential in the use of this observation to compute phase corrections. Because code and phase errors are in phase, the drawback of the PMMW based identification at very short additional distances might be able to be solved using the observed code error directly.

The amplitude $\left(\hat{\mathrm{A}}_{\mathrm{i} \backslash \text { code error }}\right)$ and argument $\left(\hat{\mathrm{h}}_{\mathrm{i} \text { \ode error }}\right)$ of this observable can be identified by the combined ANF\&ALS process, similarly as Eq. 7.12 with the signal-to-noise ratio:

$\tau=\sum_{\mathrm{i}} \hat{\mathrm{A}}_{\mathrm{i} \backslash \text { code error }} \sin \hat{\mathrm{h}}_{\mathrm{i} \backslash \text { code error }}$

A filter can be designed on the basis on this consideration. It has already been confirmed that the multipath code error can only be used to identify the argument 
(frequency and phase) of the phase error, but not its amplitude $\left(\hat{\mathrm{A}}_{\mathrm{i} \text { ।code error }}=\alpha_{\mathrm{i}}{ }^{*} \mathrm{~d}_{\mathrm{i}}\right)$ due to the unknown code delay. However, the amplitude can be set up based on the signal-tonoise ratio. In this scenario (see Fig. 7.10), the equation of reconstruction is the same as Eq. 7.15 with $\hat{\mathrm{h}}_{\mathrm{i} \mathrm{PMM} w}$ since code and phase errors are in phase:

$\varphi=1 / \mathrm{K} \sum_{\mathrm{i}} \hat{\mathrm{A}}_{\mathrm{i} \backslash \mathrm{SNR}} \sin \left(\hat{\mathrm{h}}_{\mathrm{i} \backslash \mathrm{code} \text { error }}\right)$

$\underline{\text { SNR and code error based processes }}$

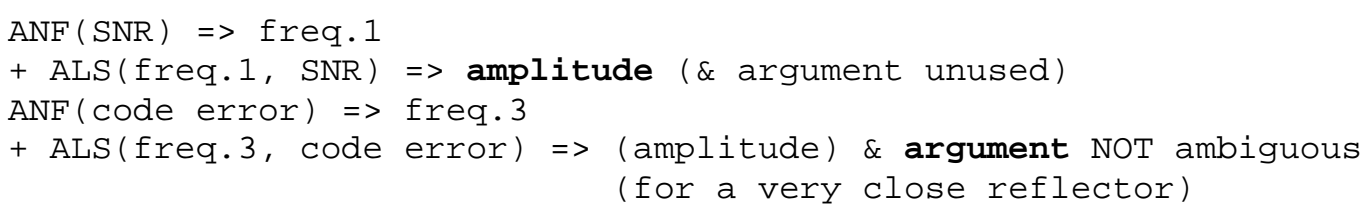

Figure 7.10: the investigated SNR and code mixed scenario

This algorithm has been tested again on SV1 and SV2 data sets collected in static mode. Fig. 7.11 displays the standard deviation of the corrected O-C DD of L1 phase versus the same variable gain $\mathrm{k} / \mathrm{K}$ applied in the phase error reconstruction processes. It is important to recall that a scenario is relevant if the gain that corresponds to the minimum standard deviation does not depend on the distance to the reflector. The "black $(+)$ ", "magenta $\left(^{*}\right)$ " and "blue (\#)" lines refer to respectively the scenarios with SNR only, with the PMMW estimate + SNR $\left(4^{\text {th }}\right.$ scenario in Fig. 7.7$)$ and lastly with the code error + SNR.

The code error + SNR mixed reconstruction algorithm is suggested here in order to take into account the specific behaviour of the code error in the case of a very close reflector. And the test at $1 \mathrm{~m}$ (see Fig. 7.11a) actually shows the potential of the code error to estimate a phase correction. The obtained results enable a standard deviation of $5 \mathrm{~mm}$, instead of 7 to $8 \mathrm{~mm}$, to be reached. So it appears that the shape of the reconstructed phase error based on the code error is closer to the O-C DD of L1 phase than that reconstructed with the PMMW, or even with the SNR. But Fig. 7.11b and 7.11c confirm that it is only relevant for very close multipath. Finally, an algorithm that is adaptive with the distance to the reflector is needed. 

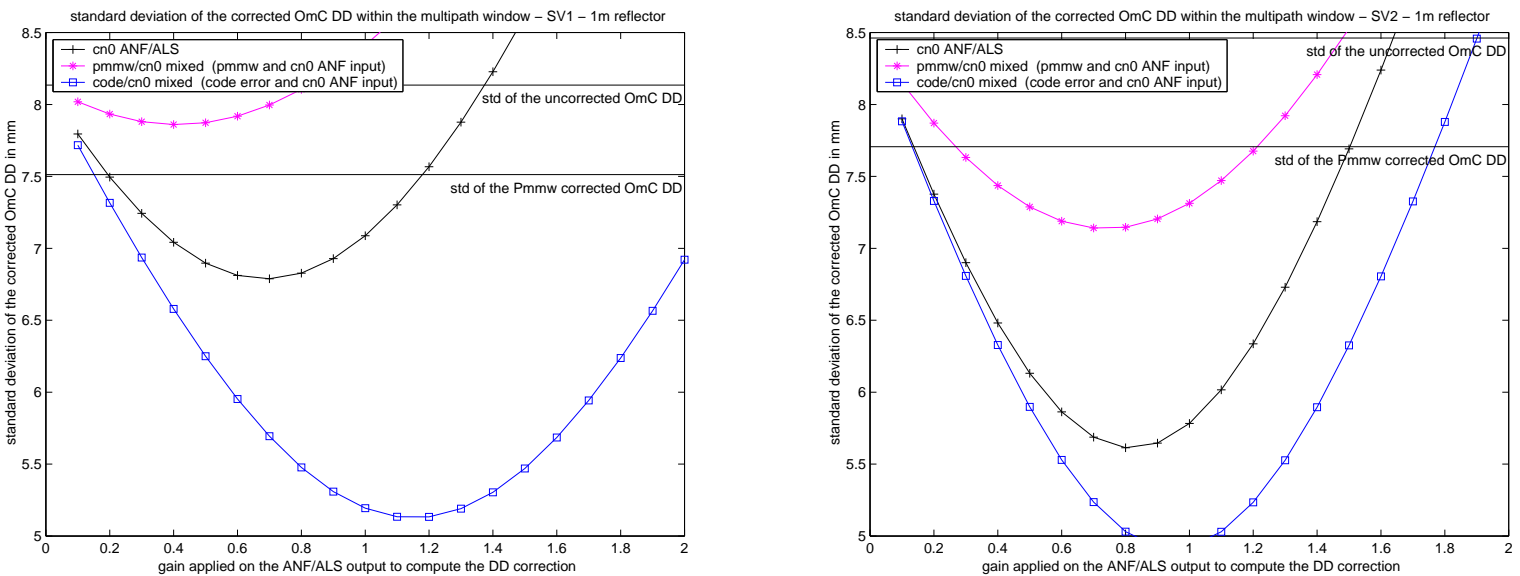

Figure 7.11a: SNR and code mixed scenario compared to SNR and PMMW/SNR mixed scenarios in the case of a very close reflector, i.e. $1 \mathrm{~m}$ distant (SV1 and SV2)
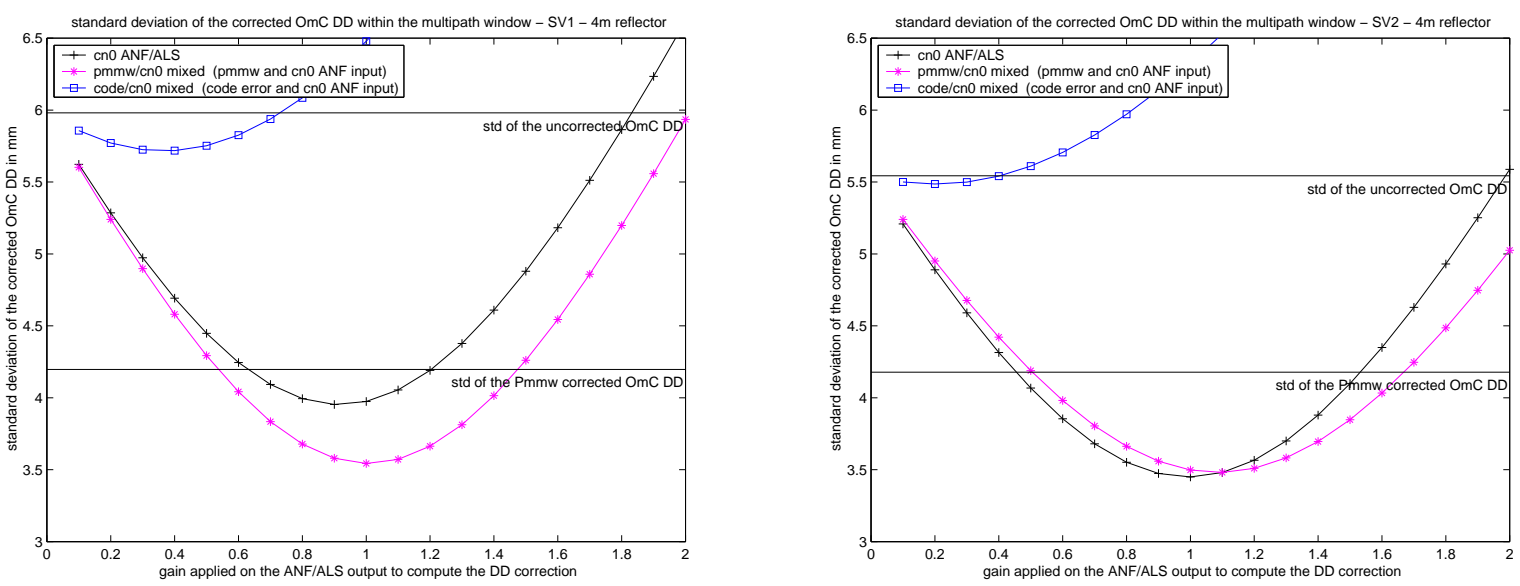

Figure 7.11b: SNR and code mixed scenario compared to SNR and PMMW/SNR mixed scenarios in the case of the $4 \mathrm{~m}$ distant reflector (SV1 and SV2)
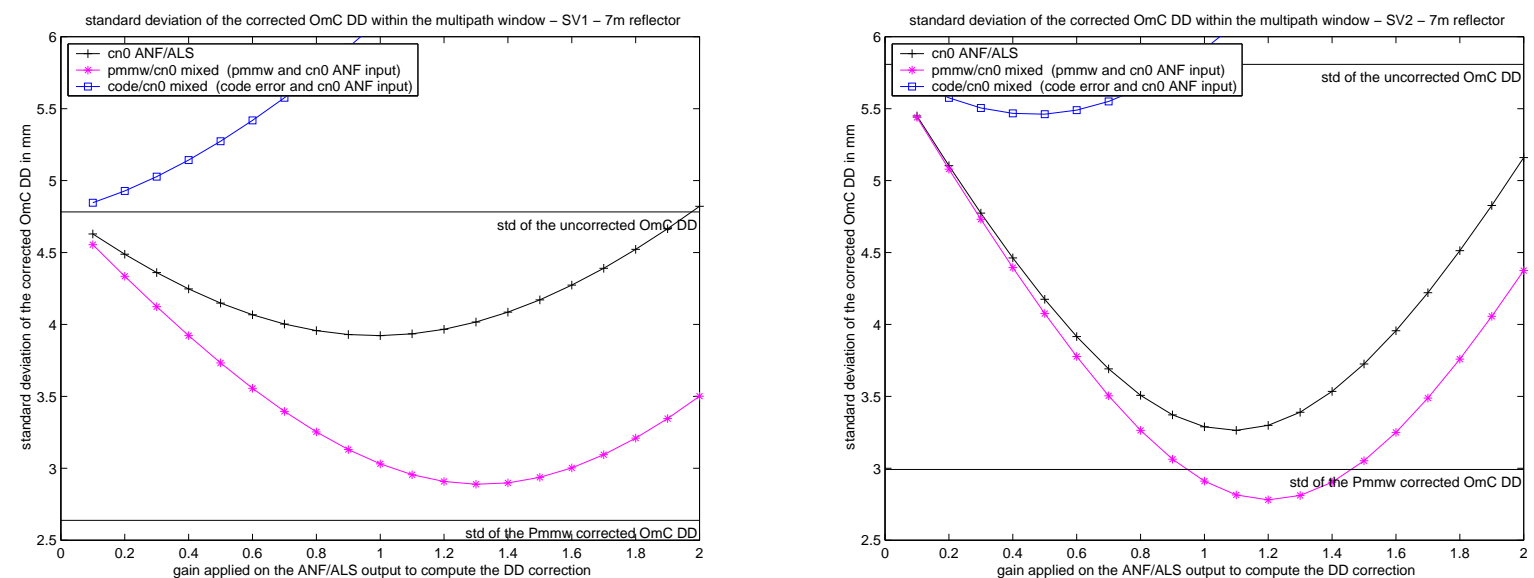

Figure 7.11c: SNR and code mixed scenario compared to SNR and PMMW/SNR mixed scenarios in the case of the $7 \mathrm{~m}$ distant reflector (SV1 and SV2) 


\subsection{4 - Fusion of the reconstruction processes}

Initial efforts at combining output from the PMMW correlator with SNR and code data suggest that SNR is always useful to determine the amplitude of the correction. However, the argument of the correction needs to be computed from either the PMMW estimation or the code error, depending on the distance to the reflector. So, it appears that the two strategies are both potentially rather efficient, but for two different situations in terms of distance to the reflector. The next step that is developed in this section is the fusion of these reconstruction processes.

An intuitive and basic idea of a possible fusion is presented here. It gives a first step in designing an adaptive algorithm. The reconstructed phase errors could be mixed with respect to the phase difference between the multipath code error and the SNR variation (and PMMW, eventually deteriorated, is unused here):

- if they are in phase, use only the phase correction computed from the SNR variation; - if they are in quadrature, use only the phase correction computed from the code error; - between these two opposite situations, balance with a weighting of the two reconstructed phase errors in the final mixing fixed linearly with respect to the phase difference between code error and SNR variation. The following ratio is introduced:

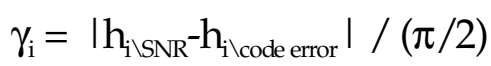

and the final reconstructed phase error results from the following combination:

$\varphi=\left(1-\gamma_{\mathrm{i}}\right) / \mathrm{K} \sum_{\mathrm{i}} \hat{\mathrm{A}}_{\mathrm{i} \backslash \mathrm{SNR}} \sin \left(\hat{\mathrm{h}}_{\mathrm{i} \backslash \mathrm{PMMN}}\right)+\left(\gamma_{\mathrm{i}}\right) / \mathrm{K} \sum_{\mathrm{i}} \hat{\mathrm{A}}_{\mathrm{i} \backslash \mathrm{SNR}} \sin \left(\hat{\mathrm{h}}_{\mathrm{i} \backslash \text { code error }}\right)$

Note that the phase MMW, although it is unused in ratio $\gamma_{i}$ is still necessary to get the sign of the correction unambiguously when the reflector is far enough. This algorithm is tested on the data sets for SV1 and SV2 already used in § 7.3.2 and § 7.3.3 (see Fig. 7.12).

Finally, Fig. 7.13 and 7.14 display the reconstructed corrections obtained from the observables already displayed in Fig. 7.5, through the algorithm given in Eq. 7.19. 

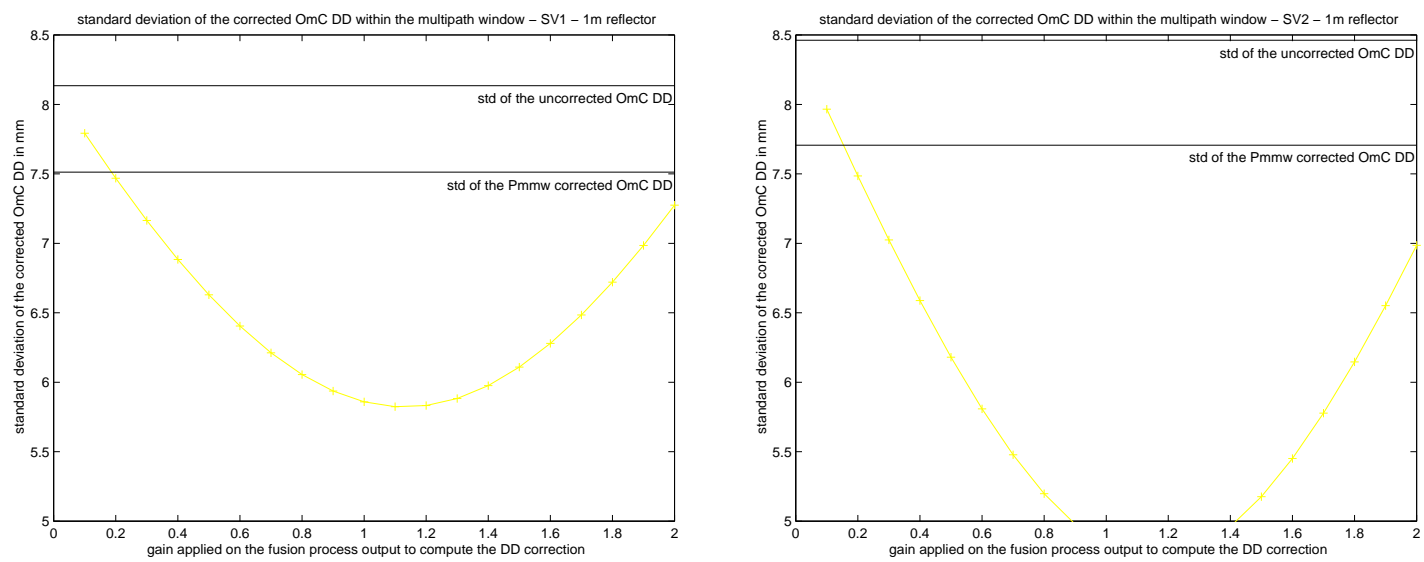

Figure 7.12a: SNR, code error and PMMW mixed scenario in the case of a very close reflector, i.e. $1 \mathrm{~m}$ distant (SV1 and SV2)
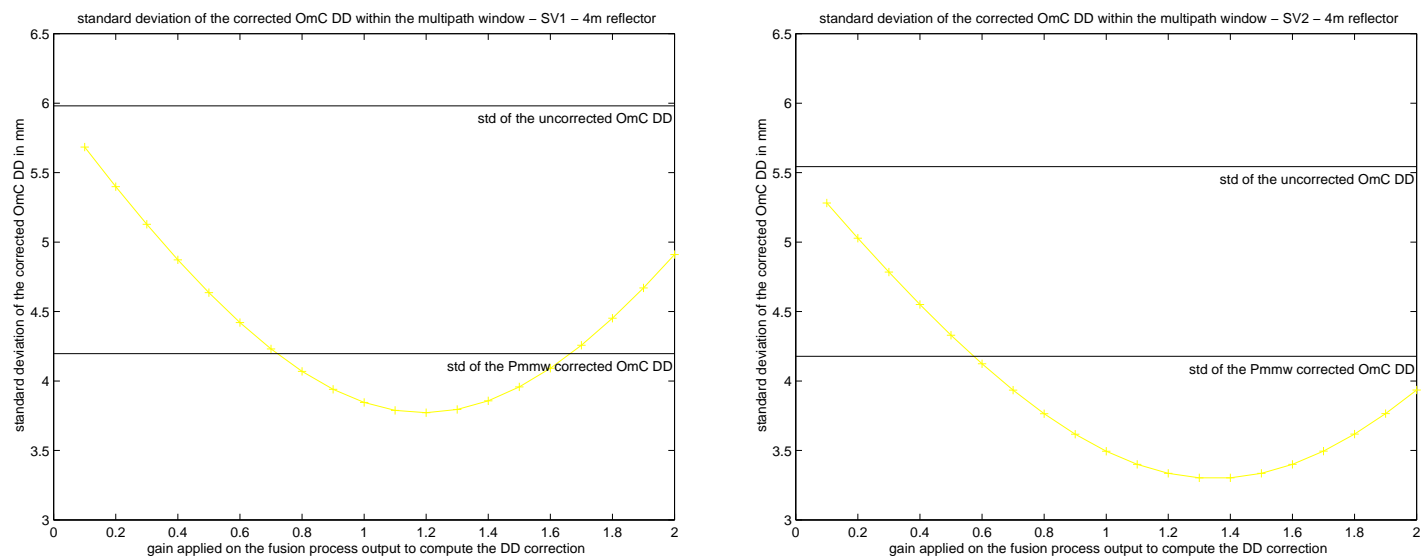

Figure 7.12b: SNR, code error and PMMW mixed scenario in the case of the $4 \mathrm{~m}$ distant reflector (SV1 and SV2)
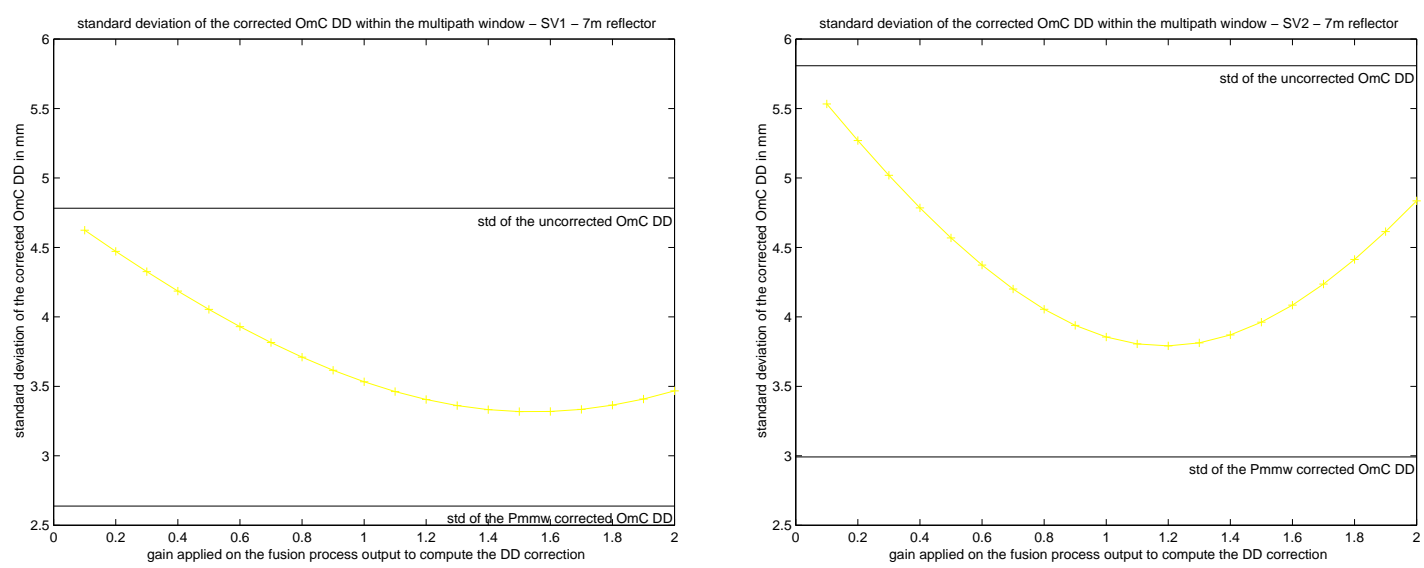

Figure 7.12c: SNR, code error and PMMW mixed scenario in the case of the $7 \mathrm{~m}$ distant reflector (SV1 and SV2) 


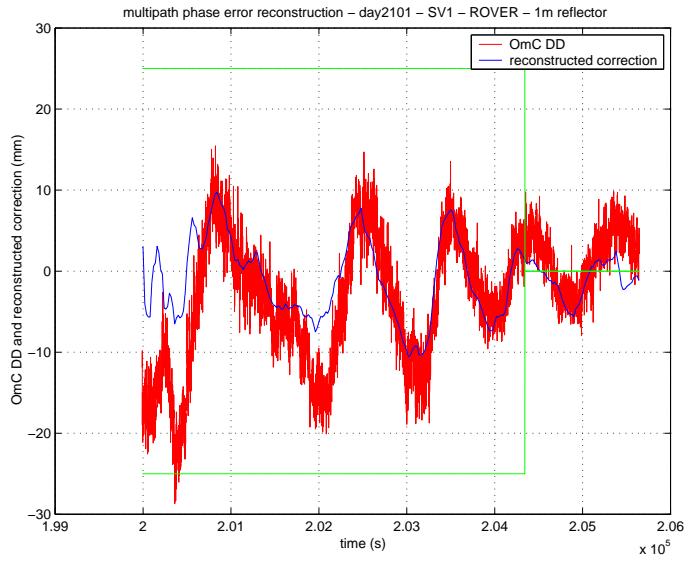

Figure 7.13a: reconstructed error (SV1 $1 \mathrm{~m}$ test)

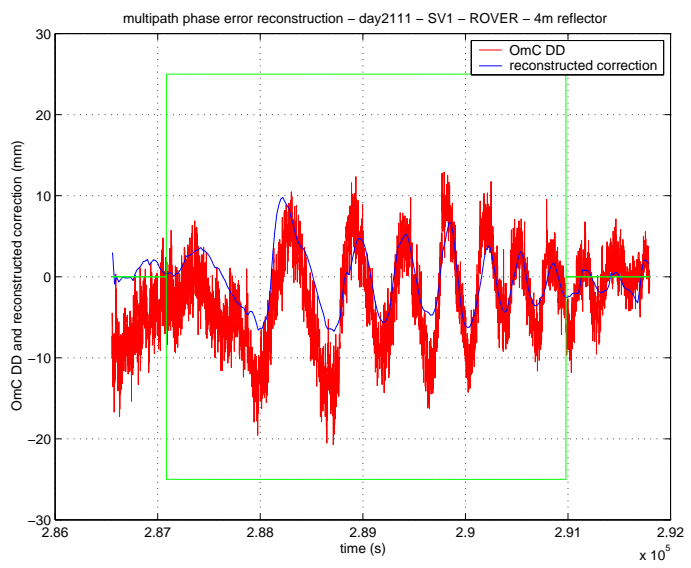

Figure 7.13b: reconstructed error (SV1 $4 \mathrm{~m}$ test)

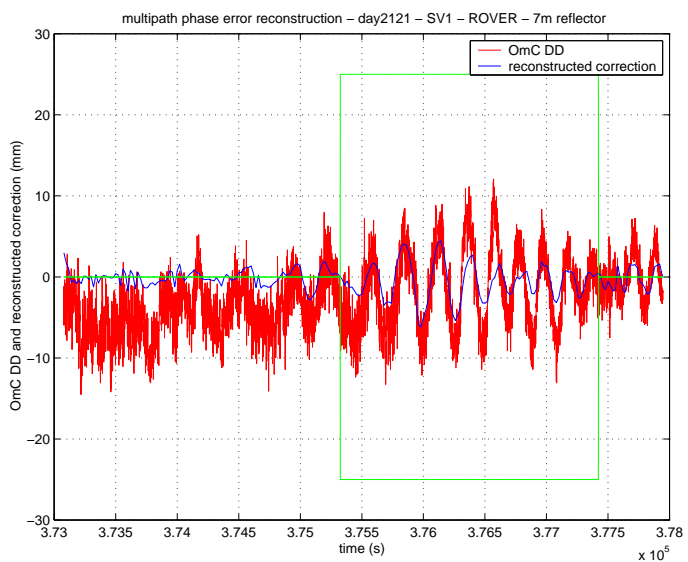

Figure 7.13c: reconstructed error (SV1 $7 \mathrm{~m}$ test))

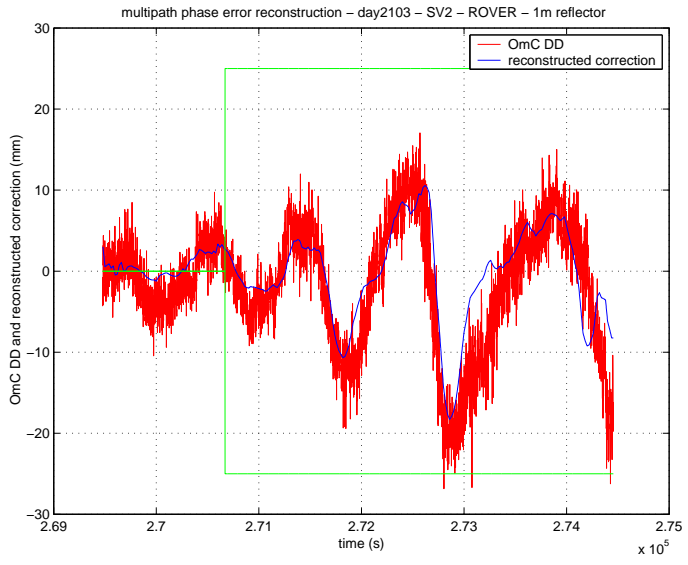

Figure 7.14a: reconstructed error (SV2 $1 \mathrm{~m}$ test)

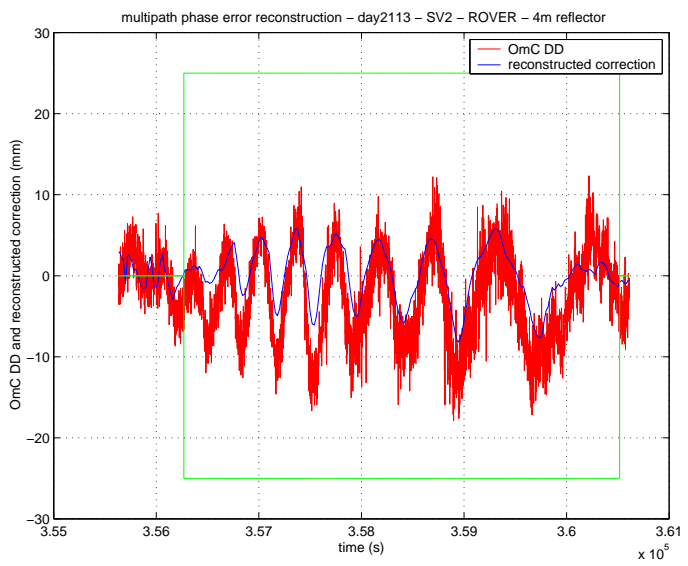

Figure 7.14b: reconstructed error (SV2 $4 \mathrm{~m}$ test)

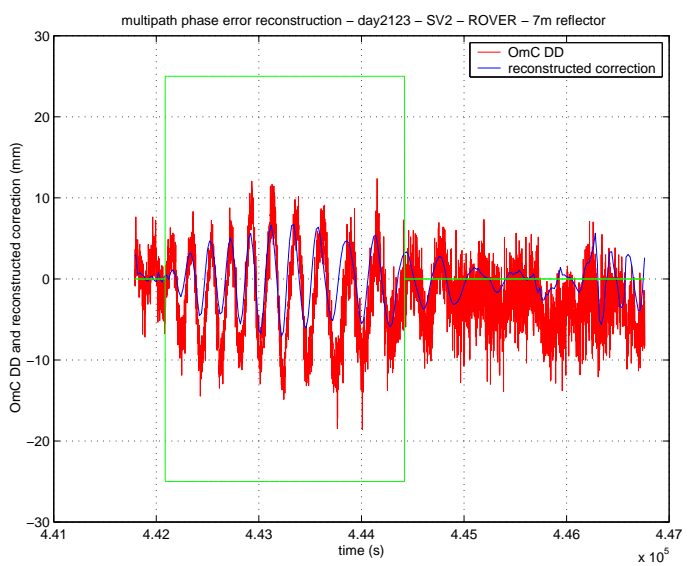

Figure 7.14c: reconstructed error (SV2 $7 \mathrm{~m}$ test) 
From visual inspection on Fig. 7.13, it actually seems that this algorithm, at least on the two selected satellites, gives significantly improved results when the reflector is at $1 \mathrm{~m}$ compared to those obtained by a PMMW direct correction. This algorithm has the advantage, contrary to the preceding ones, to adapt to the close reflector situation, while remaining pretty efficient when the reflector gets further away (4 and $7 \mathrm{~m}$ during the present tests). Note that at $4 \mathrm{~m}$, the results are still the best running this algorithm, and at $7 \mathrm{~m}$, the PMMW direct correction gets the most efficient.

On these two satellites, the optimal gain tuning is again around unity, i.e. a reconstruction using $K=20 / \ln (10)$, which means that the modelling fits pretty well the observations. However, the sharpness of the parabolic curve reduces as the distance to the reflector increases, which is symptomatic of the limitation of the modelling chosen.

It is now interesting to generalise the application of this algorithm to the entire data sets collected during the second campaign of tests. This is done in next section.

\section{4- Generalisation of the application of the algorithm}

\subsection{1 - Processing issues}

There is a priori no way to select geometrically the satellites that are affected by multipath in an unknown environment. If the environment is well known, like in the case of the SESSYL platform discussed in Appendix 5, then geometrical modelling is possible.

Here, the only multipath detection relies on the code error observable, the SNR and the PMMW outputs, which are precisely the inputs of the algorithm that this thesis suggests. Hence, this algorithm will be run continuously, for every satellite, for every epoch. The problem in real-time of the averaging of the code error observable is only treatable by using a moving window, which of course entails a certain latency. The results given further can only obtained in post-processing. They were actually obtained for every satellite by averaging the code error observable over series of epochs showing no loss of lock of this satellite. 
Besides the real-time averaging issue of the multipath code error, another issue comes from the double-differencing process that outputs the O-C DD of L1 phase and that needs to be computed again. It is essential that one should keep in mind that DD will mix the reconstructed phase corrections of all satellites, including the differencing ones with the opposite sign. Actually, there is again no a priori reason why the correction should not be applied to the differencing satellite, except if it is certain that no reflector will cause multipath at high elevations. This modifies noticeably the reconstructed errors through the incorporation of additional noise (see Fig. 7.15 and 7.16).

\subsection{2 - Static data}

Tables 7.2a, 7.2b and 7.2c are similar as tables $6.5 a, 6.5 b$ and $6.5 c$ for respectively day210, day211 and day212, with the panel placed at 1, 4 and $7 \mathrm{~m}$. The statistics given are again standard deviations of O-C DD of L1 phase in multipath window time zones.

Note:

sdn standard deviation of the O-C DD of L1 "reconstructed" phase in $\mathrm{mm}$, i.e. once applied the correction algorithm. The corresponding gain in \% is computed with respect to the standard deviation of the O-C DD of L1 "standard" phase.

\begin{tabular}{rrrrrrr} 
sv & dura & sd & \multicolumn{2}{c}{ sdx } & gain\% & \multicolumn{2}{c}{ sdn } & gain\% \\
1 & 5697 & 9.3 & 8.6 & 7.3 & 8.0 & 14.1 \\
2 & 7178 & 10.5 & 9.4 & 10.8 & 7.6 & 28.1 \\
3 & 8066 & 10.5 & 9.6 & 8.3 & 9.5 & 9.3 \\
4 & 7197 & 9.0 & 8.5 & 5.1 & 7.4 & 17.6 \\
5 & 6439 & 9.9 & 9.8 & 1.0 & 10.6 & -7.5 \\
6 & 6766 & 9.1 & 8.4 & 7.8 & 8.3 & 9.5 \\
7 & 6435 & 11.9 & 12.3 & -4.0 & 10.1 & 14.7 \\
8 & 6942 & 8.0 & 7.6 & 4.8 & 7.3 & 8.8 \\
9 & 9271 & 10.7 & 11.2 & -5.1 & 12.6 & -18.2 \\
10 & 6734 & 8.0 & 7.7 & 3.5 & 6.5 & 18.2 \\
11 & 9537 & 12.1 & 11.7 & 3.2 & 12.1 & -0.2 \\
13 & 6799 & 8.4 & 7.9 & 6.2 & 6.7 & 20.9 \\
14 & 6410 & 12.5 & 12.3 & 1.3 & 11.9 & 4.5 \\
15 & 6586 & 9.8 & 8.8 & 10.6 & 7.2 & 26.3 \\
16 & 6831 & 11.9 & 10.4 & 12.6 & 10.0 & 15.6 \\
17 & 8525 & 11.8 & 11.7 & 0.2 & 13.1 & -11.8 \\
18 & 7812 & 10.4 & 11.5 & -10.3 & 12.7 & -22.5 \\
20 & 6532 & 15.0 & 13.9 & 7.7 & 15.6 & -4.2 \\
21 & 6092 & 8.7 & 7.5 & 13.9 & 6.6 & 24.1
\end{tabular}




\begin{tabular}{|c|c|c|c|c|c|}
\hline 236886 & 9.5 & 9.2 & 3.3 & 9.5 & 0.3 \\
\hline 46918 & 8.9 & 9.3 & -4.6 & 8.8 & 1.4 \\
\hline 7068 & 10.5 & 9.9 & 5.5 & 8.9 & 14.6 \\
\hline 6549 & 9.2 & 7.9 & 14.0 & 8.5 & 7.6 \\
\hline 685 & 12.1 & 10.7 & 11.4 & 10.3 & 14.9 \\
\hline 799 & 12.6 & 12.5 & 0.9 & 12.8 & -1.7 \\
\hline 6886 & 10.6 & 11.6 & -9.4 & 12.9 & -21.0 \\
\hline 5845 & 9.1 & 7.8 & 13.8 & 6.6 & 27.6 \\
\hline & 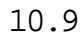 & & 4. & 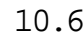 & \\
\hline
\end{tabular}

Table 7.2a: statistical results of the reconstruction for day 210 (panel at $1 \mathrm{~m}$ )

Note: the same table (table 7.2a*) has been computed again after removing the data corresponding to low elevated satellites $\left(15^{\circ}\right.$ threshold) that are included in the multipath window time zones. Such data do not exist for the tests at 4 or $7 \mathrm{~m}$.

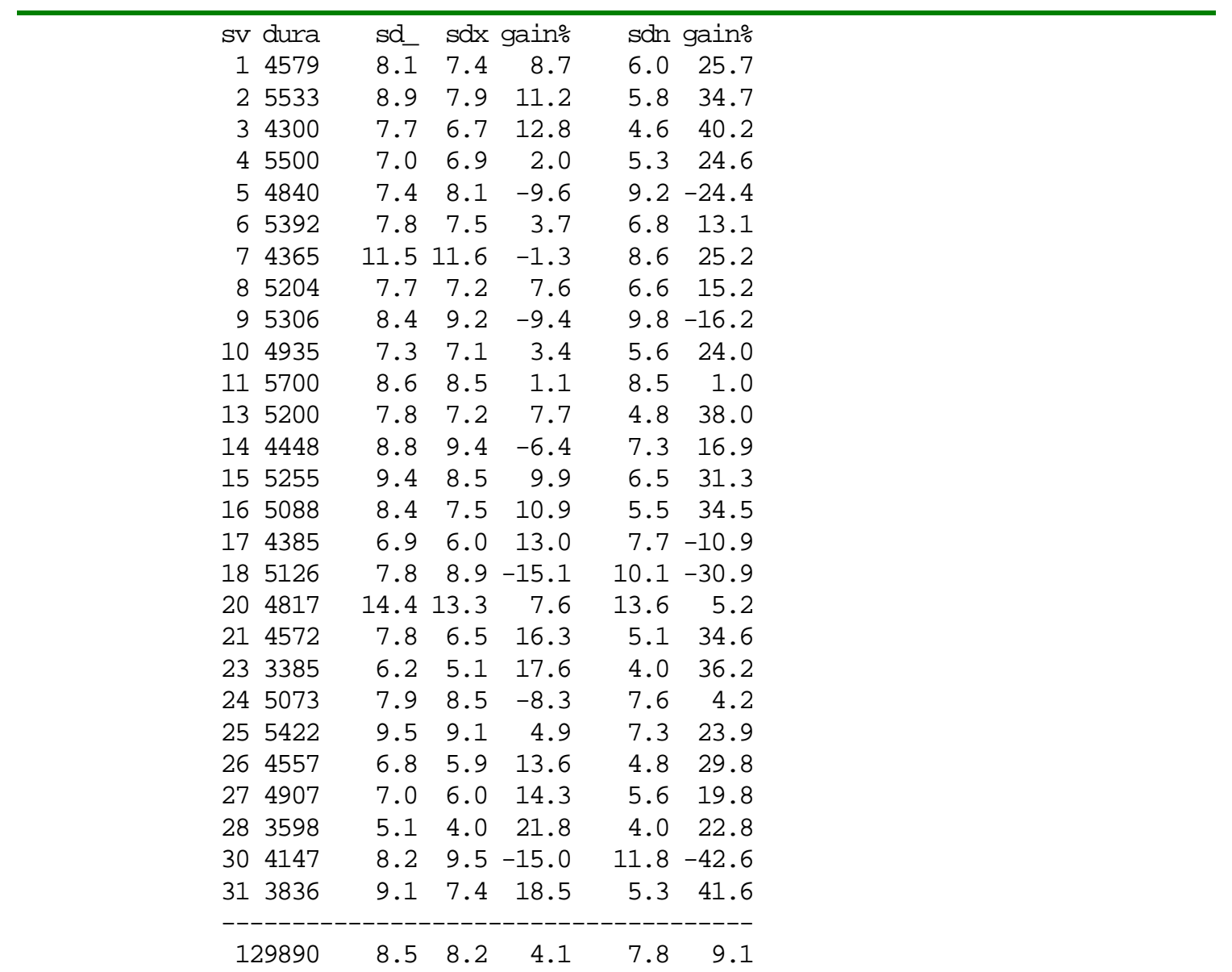

Table 7.2a* : same as table 7.2a, but SVS with elevation $>15^{\circ}$ only are included 
Both tables 7.2a and 7.2a* show "negative" gains that often match those in table 6.3a. This is not unexpected since the reconstruction process uses PMMW amongst other observables. As already mentioned in Chapter $6, \S 6.3$, this also generally corresponds to multipath with the smallest additional path lengths.

\begin{tabular}{|c|c|c|c|c|c|}
\hline SV & dura & sd_ sdx & gaino & sdn & gain $\%$ \\
\hline & 3896 & $6 . \overline{0} 4.2$ & 29.8 & 3.9 & 34.3 \\
\hline & 4249 & 5.54 .2 & 24.6 & 3.6 & 35.9 \\
\hline & 4183 & 5.24 .0 & 22.9 & 3.8 & 26.5 \\
\hline & 3768 & $5.4 \quad 4.7$ & 13.3 & 4.8 & 11.8 \\
\hline 6 & 4086 & 5.14 .1 & 20.3 & 3.8 & 25.1 \\
\hline 7 & 87 & 3.73 .7 & -0.6 & 3.5 & 2.8 \\
\hline & 3959 & 4.73 .6 & 23.6 & 3.3 & 30.5 \\
\hline & 3172 & 4.83 .9 & 19.2 & 3.6 & 24.9 \\
\hline 13 & 3923 & 5.94 .4 & 25.1 & 4.2 & 28.0 \\
\hline 14 & 1017 & 3.53 .3 & 6.3 & 3.9 & -12.1 \\
\hline & 3973 & 6.75 .4 & 18.5 & 5.2 & 22.4 \\
\hline & 4039 & 4.23 .2 & 23.3 & 3.1 & 27.0 \\
\hline & 3733 & 5.74 .8 & 15.6 & 5.1 & 9.7 \\
\hline & 3642 & 4.13 .3 & 18.8 & 3.6 & 12.4 \\
\hline 24 & 3834 & 5.95 .0 & 14.5 & 4.4 & 25.0 \\
\hline & 4086 & 6.75 .9 & 11.9 & 5.2 & 23.1 \\
\hline & 3771 & 4.03 .0 & 25.9 & 3.3 & 16.8 \\
\hline & 3851 & 5.13 .6 & 28.1 & 3.5 & 30.8 \\
\hline 31 & 4004 & $5.2 \quad 3.4$ & 34.7 & 3.7 & 28.1 \\
\hline & 68214 & $5.4 \quad 4.3$ & 20.4 & 4.3 & 20.6 \\
\hline
\end{tabular}

Table 7.2b: statistical results of the reconstruction for day211 (panel at $4 \mathrm{~m}$ )

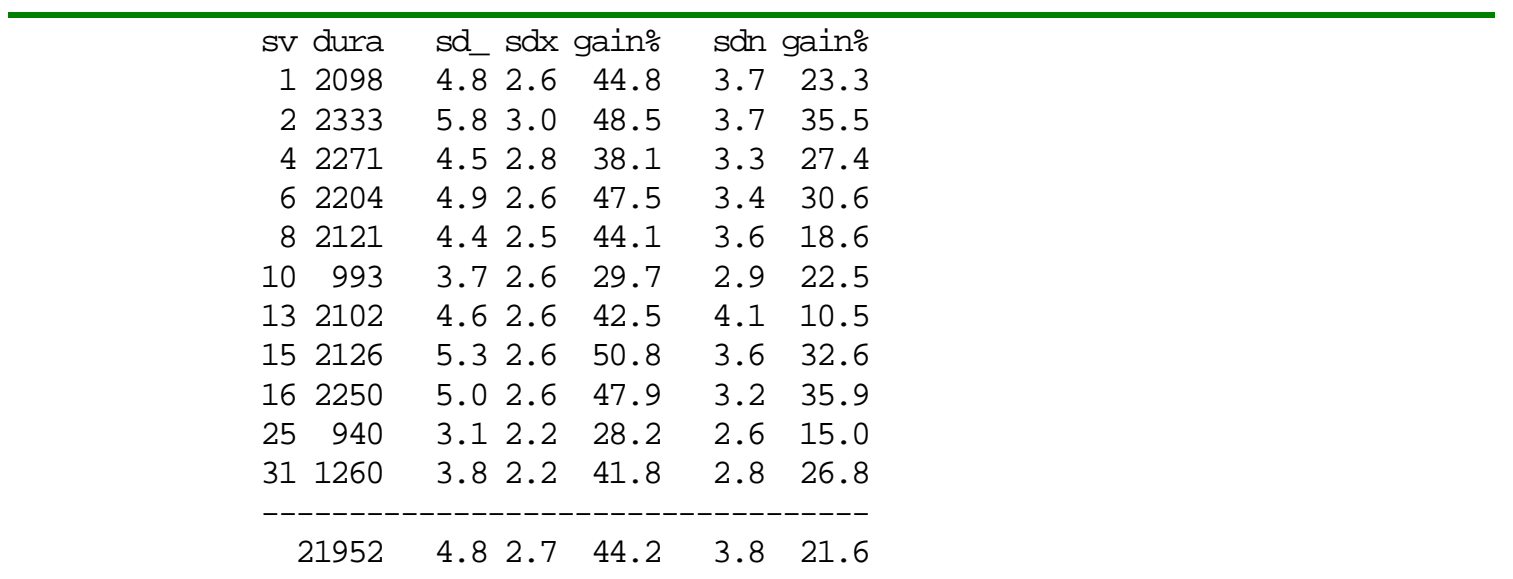

Table 7.2c: statistical results of the reconstruction for day212 (panel at $7 \mathrm{~m}$ ) 

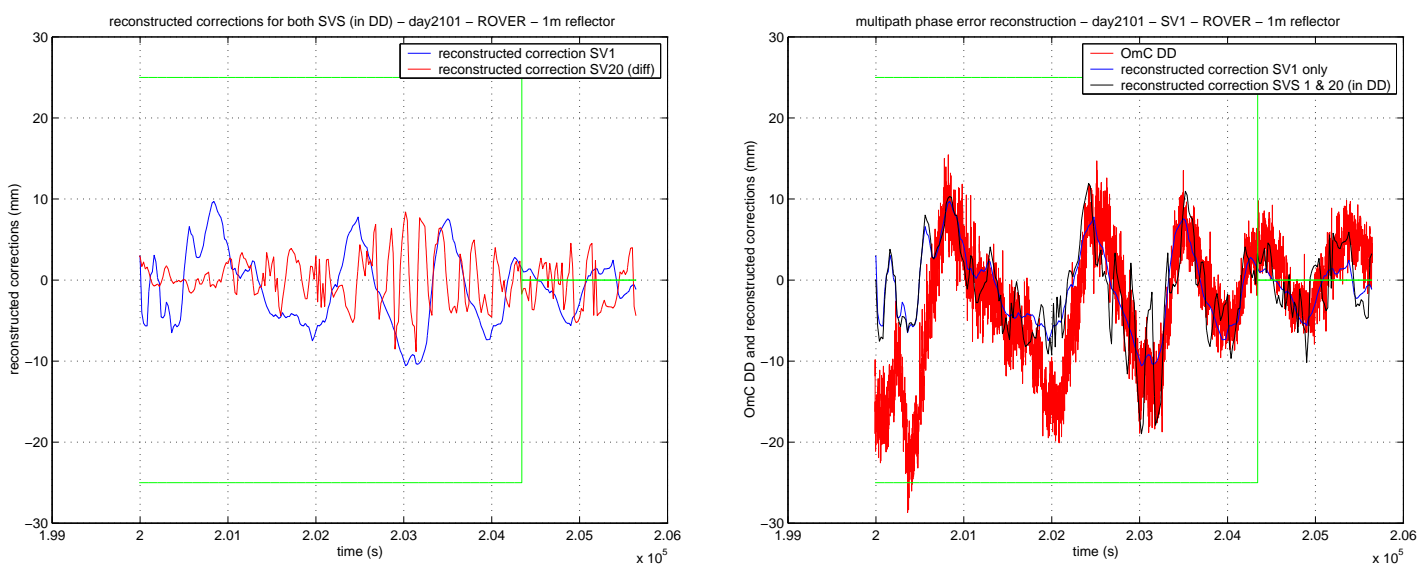

Figure 7.15a: reconstructed multipath phase errors for SV1 and differencing SV20 (left figure) and combination of both in a global correction of the O-C DD of phase ( $1 \mathrm{~m}$ test)
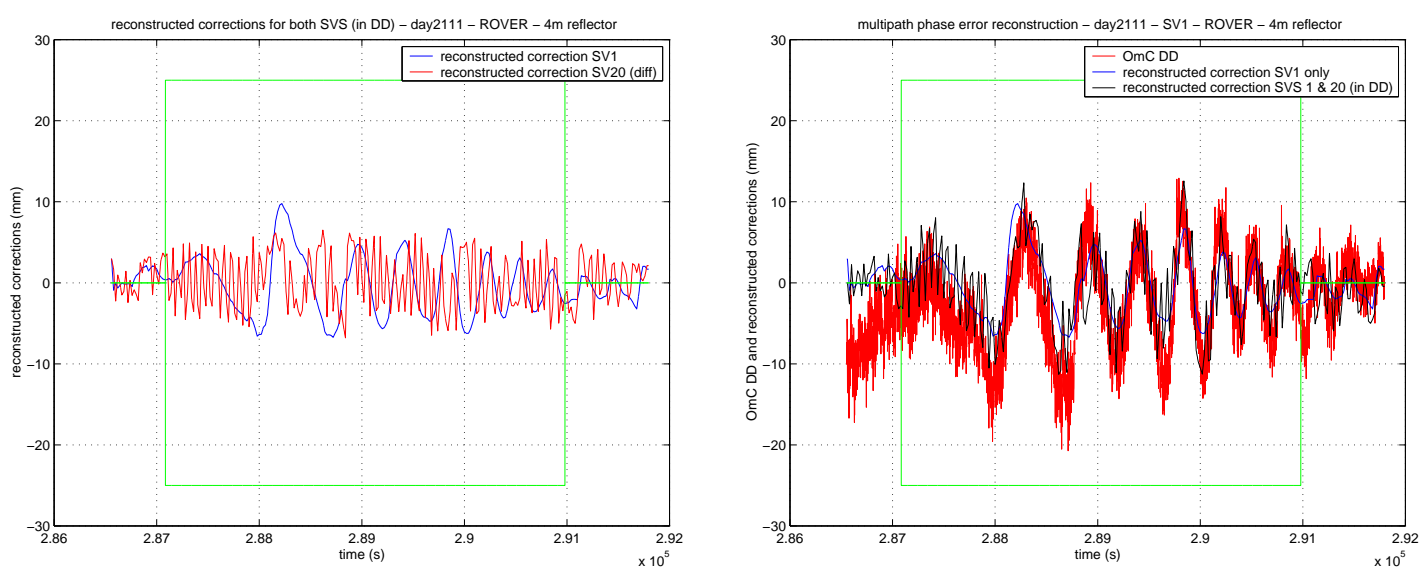

Figure 7.15b: reconstructed multipath phase errors for SV1 and differencing SV20 (left figure) and combination of both in a global correction of the O-C DD of phase (4 $\mathrm{m}$ test)
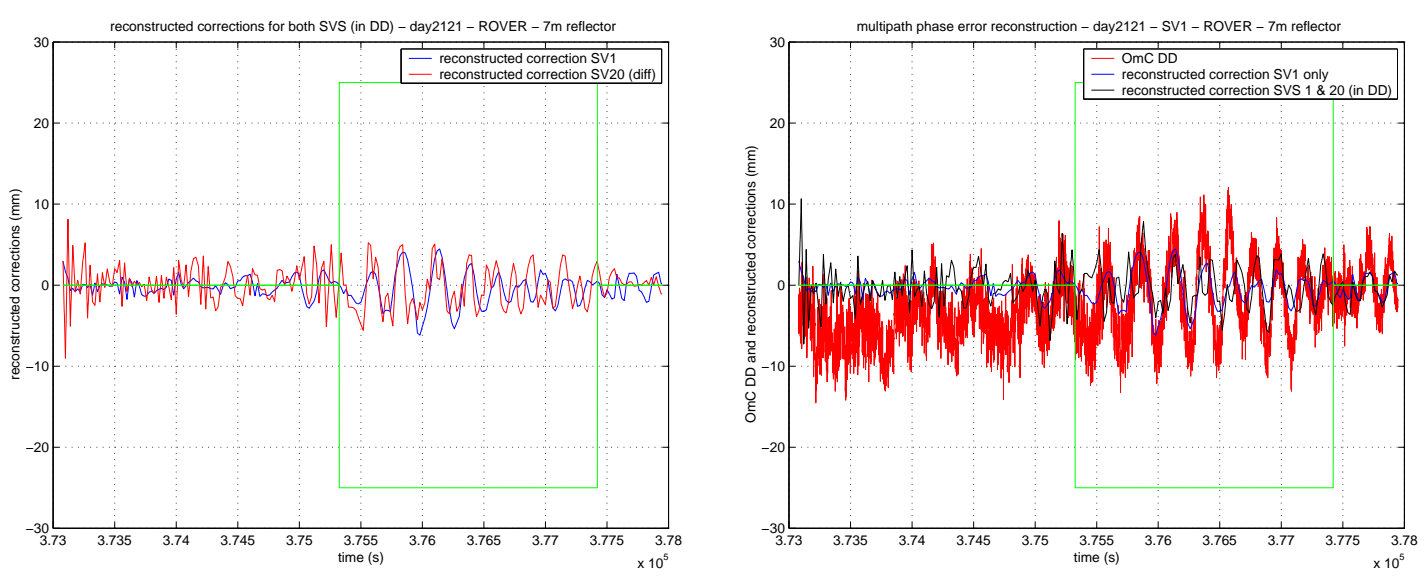

Figure 7.15c: reconstructed multipath phase errors for SV1 and differencing SV20 (left figure) and combination of both in a global correction of the O-C DD of phase (7 m test) 

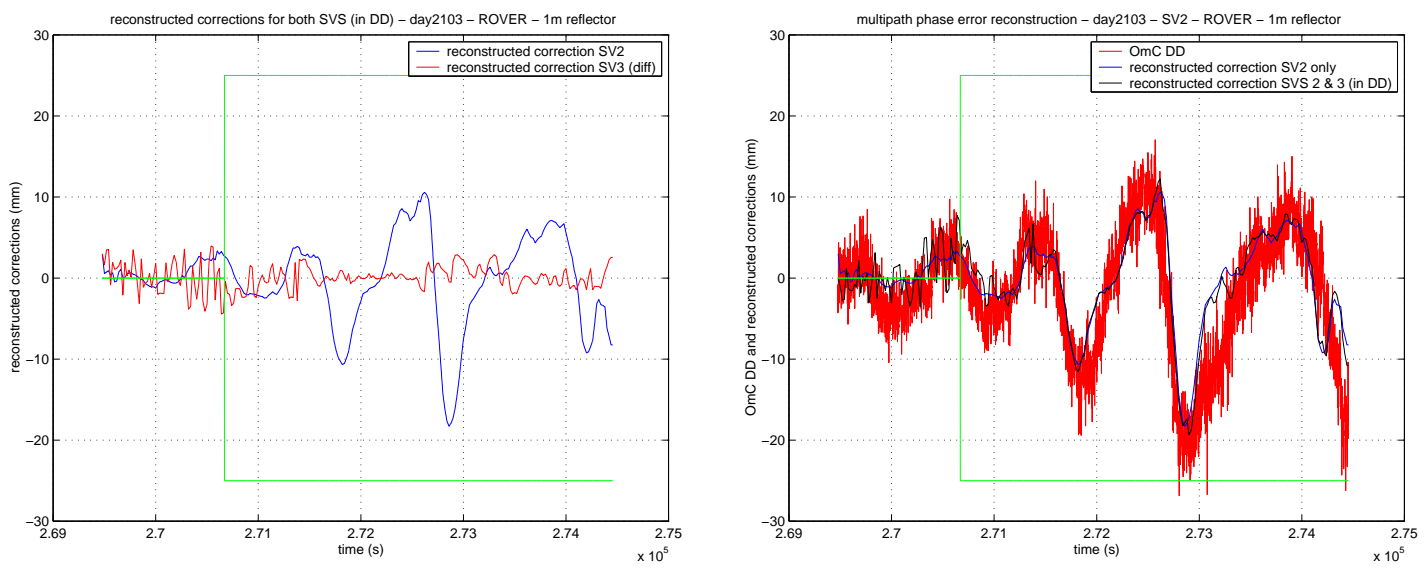

Figure 7.16a: reconstructed multipath phase errors for SV2 and differencing SV3 (left figure) and combination of both in a global correction of the O-C DD of phase ( $1 \mathrm{~m}$ test)
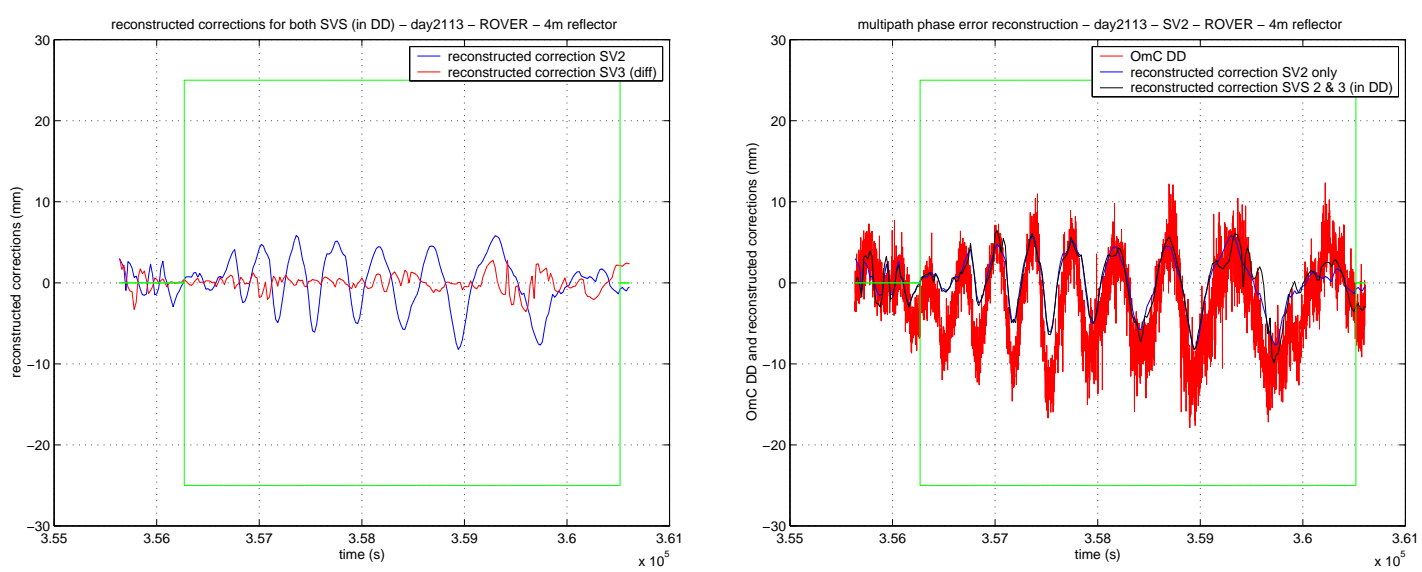

Figure 7.16b: reconstructed multipath phase errors for SV2 and differencing SV3 (left figure) and combination of both in a global correction of the O-C DD of phase (4 $\mathrm{m}$ test)
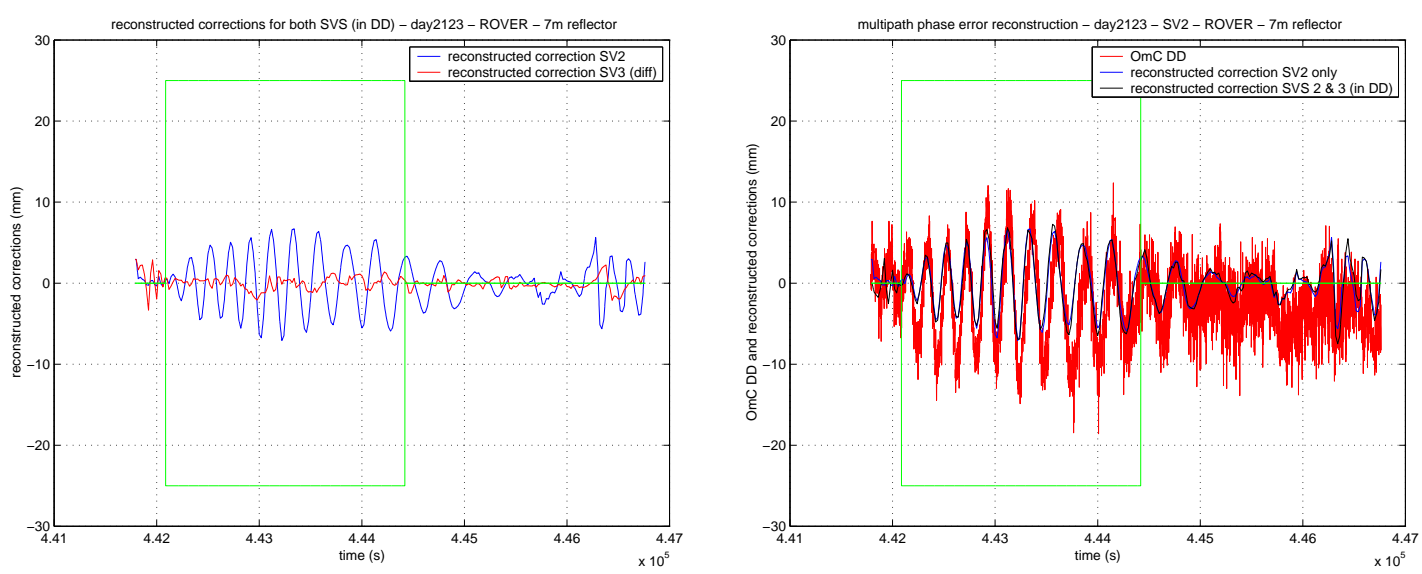

Figure 7.16c: reconstructed multipath phase errors for SV2 and differencing SV3 (left figure) and combination of both in a global correction of the O-C DD of phase (7 m test) 
Fig. 7.15 and 7.16 illustrate that the contribution of the differencing data in the multipath correction process is sensitive, and not necessarily relevant (like for differencing SV20 that shows a rather large correction although no significant multipath exist on this satellite, but simply more noise in data).

The results of the reconstruction process in static mode are summarised in table 7.3 and displayed in bold, for all satellites and all multipath zones. The preceding results (see Chapter 6, table 6.4) corresponding to the standard measurements and those modified by the PMMW corrections are given for memory.

\begin{tabular}{cccc}
\hline Static tests & $\sigma$ & $\sigma_{\text {PMMW }}$ & Gain \\
& & $\sigma_{\text {reconstruction }}$ & \\
\hline $\begin{array}{c}\text { reflector } 1 \mathrm{~m} \\
0.7 \mathrm{~m}<\text { addpath }<2.0 \mathrm{~m}\end{array}$ & $10.9 \mathrm{~mm}$ & $10.4 \mathrm{~mm}$ & $4 \%$ \\
$\begin{array}{c}\text { reflector } 1 \mathrm{~m} \\
\text { elevation of satellites }>15^{\circ}\end{array}$ & $8.5 \mathrm{~mm}$ & $10.6 \mathrm{~mm}$ & $3 \%$ \\
& & $8.2 \mathrm{~mm}$ & $4 \%$ \\
$\begin{array}{c}\text { reflector } 4 \mathrm{~m} \\
5.2 \text { m }<\text { addpath }<7.3 \mathrm{~m}\end{array}$ & $5.4 \mathrm{~mm}$ & $4.3 \mathrm{~mm}$ & $9 \%$ \\
\hline $\begin{array}{c}\text { reflector } 7 \mathrm{~m} \\
\mathrm{~m}<\text { addpath }<11.9 \mathrm{~m}\end{array}$ & $4.8 \mathrm{~mm}$ & $4.3 \mathrm{~mm}$ & $20 \%$ \\
\hline
\end{tabular}

Table 7.3: L1 phase DD statistics in static mode (all data in multipath zones)

(for "standard", "PWWM corrected" and "reconstructed" phase data)

The main conclusion that table 7.3 offers is that, even if the reconstruction of the multipath phase error is particularly consistent for several satellites (e.g. SV2 and SV1 in a lesser extent), it is not for a number of others, which entails a global result for very close 
reflectors that is only slightly better with the reconstruction than with the direct PMMW correction (9\% against $4 \%$, when low elevation satellites are removed). A main drawback of the reconstruction (with respect to the direct PMMW correction) is also shown when the reflector is far.

Note: the reconstruction process has also been run with the full definition of the signal-to-noise ratio (and not with a $1 \mathrm{~dB}$ binned SNR), but the results are about the same. In [BARNES, 2000], the hypothesis that a better resolution of the SRN could improve the results was suggested, but it unfortunately seems that the method is not that sensitive to the resolution: at least $1 \mathrm{~dB}$ is sufficient.

Lastly, table 7.4 gives globally the standard deviation of the O-C DD of L1 phase, for all satellites and all epochs, irrespective of the multipath zones.

\begin{tabular}{llll}
\hline Static tests & $\sigma$ & $\sigma_{\mathrm{PMMW}}$ & $\sigma_{\text {reconstruction }}$ \\
\hline reflector $1 \mathrm{~m}$ & $13.1 \mathrm{~mm}$ & $13.1 \mathrm{~mm}$ & $13.5 \mathrm{~mm}$ \\
elevation of satellites $>15^{\circ}$ & $11.8 \mathrm{~mm}$ & $11.9 \mathrm{~mm}$ & $12.2 \mathrm{~mm}$ \\
reflector $4 \mathrm{~m}$ & $8.0 \mathrm{~mm}$ & $7.8 \mathrm{~mm}$ & $8.2 \mathrm{~mm}$ \\
elevation of satellites $>15^{\circ}$ & $4.4 \mathrm{~mm}$ & $4.0 \mathrm{~mm}$ & $4.5 \mathrm{~mm}$ \\
reflector $7 \mathrm{~m}$ & $7.3 \mathrm{~mm}$ & $7.1 \mathrm{~mm}$ & $7.6 \mathrm{~mm}$ \\
elevation of satellites $>15^{\circ}$ & $4.0 \mathrm{~mm}$ & $3.7 \mathrm{~mm}$ & $4.3 \mathrm{~mm}$ \\
\hline
\end{tabular}

Table 7.4: L1 phase DD statistics in static mode (all data, all epochs) (for "standard", "PWWM corrected" and "reconstructed" phase data)

In table 7.4, the three data sets are compared (in DD): standard data, PMMW corrected data, and data where multipath phase errors have been reconstructed. It is clear that the reconstruction process brings about noise, whereas the PMMW correction reduces it (see Chapter 6, § 6.5). 


\subsection{3 - Kinematic data}

The application of the reconstruction process in the case of kinematic tests raises the issue of choosing the sampling frequency at which the ANF/ALS will be run. The time spent in front of the panel is less than 2 minutes, and almost all the multipath window time zones are concentrated within this short period of time. Obviously, a $20 \mathrm{~s}$ sampling as was used in the static tests is not adequate here, but applying the reconstruction process at every 1 s epoch seems consistent.

Except this difference in sampling, kinematic and static algorithms process data in exactly the same way.

Fig. 7.17 displays the multipath observables. From visual inspection, it is confirmed that their behaviour is similar as in the case of static tests: SNR and multipath code error are in phase and both in quadrature with the multipath phase error if the reflector is far enough. But multipath code and phase errors becomes progressively in phase for very close reflector.

However, one can notice that the magnitude of the various observables in kinematic mode is reduced compared to those in static mode (see Fig. 7.5). Note that this is also true as concerns the multipath phase error to correct. Of course, a reduction in magnitude of the useful signals (for a level of noise that keeps globally the same) is critical in the reconstruction process.

Fig. 7.18 illustrates (with SV31 and SV11 differencing) a data set for which the application of the algorithm is rather relevant.

Table 7.5a, 7.5b and 7.5c generalise the process satellite per satellite and statistics are computed on several concatenated multipath zones. 


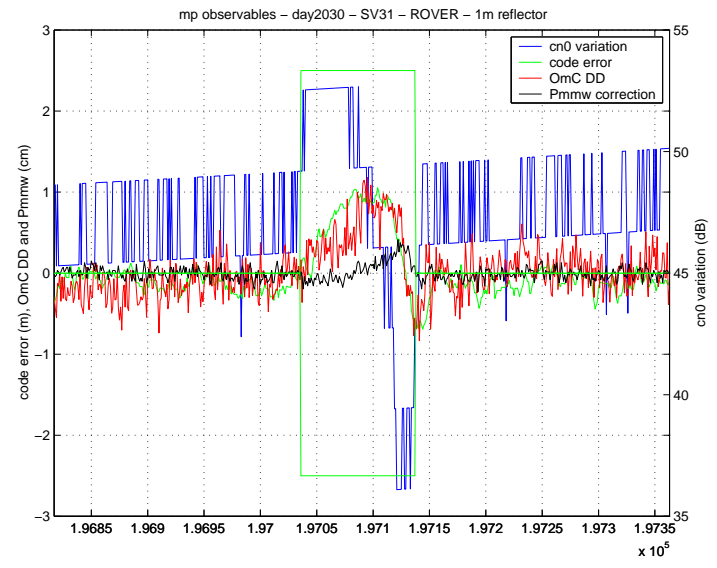

Figure 7.17a: observables and O-C L1 DD (1 m)

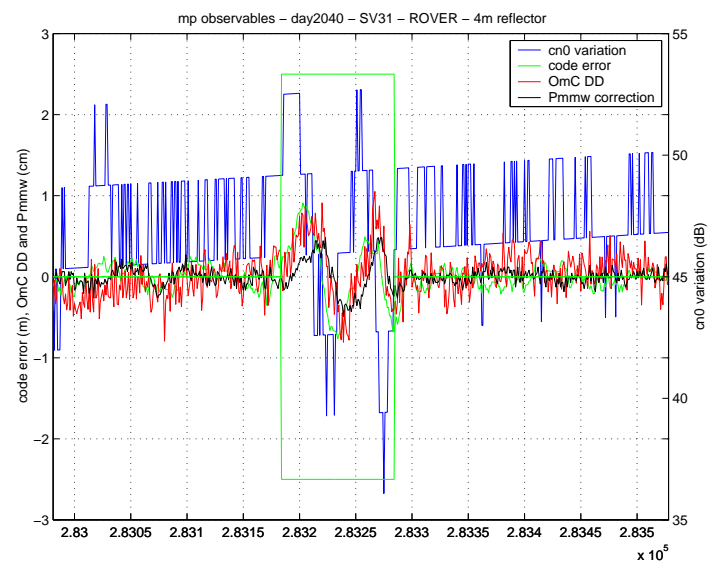

Figure 7.17b: observables and O-C L1 DD (4 m)

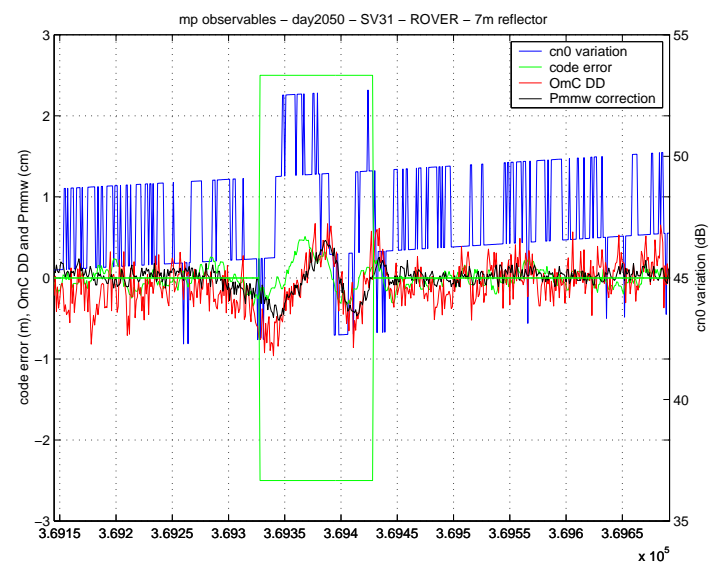

Figure 7.17c: observables and O-C L1 DD (7 m)

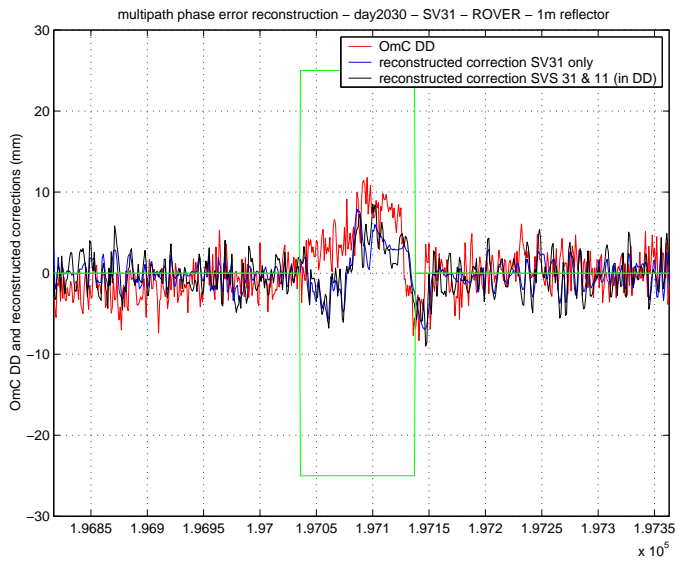

Figure 7.18a: reconstructed multipath error $(1 \mathrm{~m})$

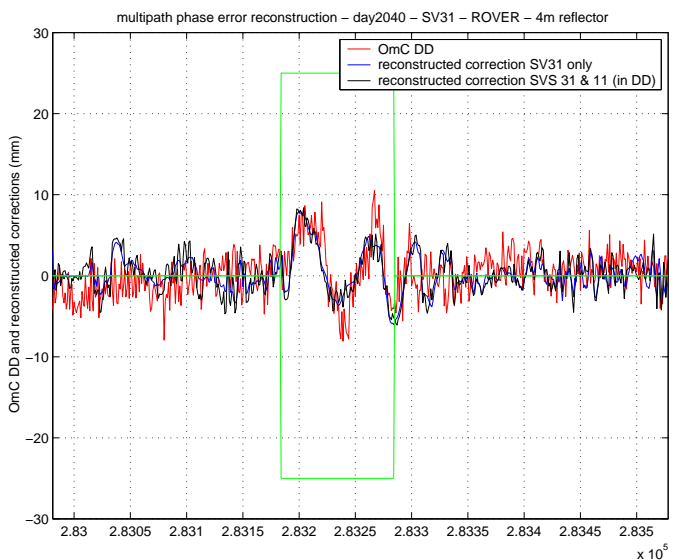

Figure 7.18b: reconstructed multipath error (4 m)

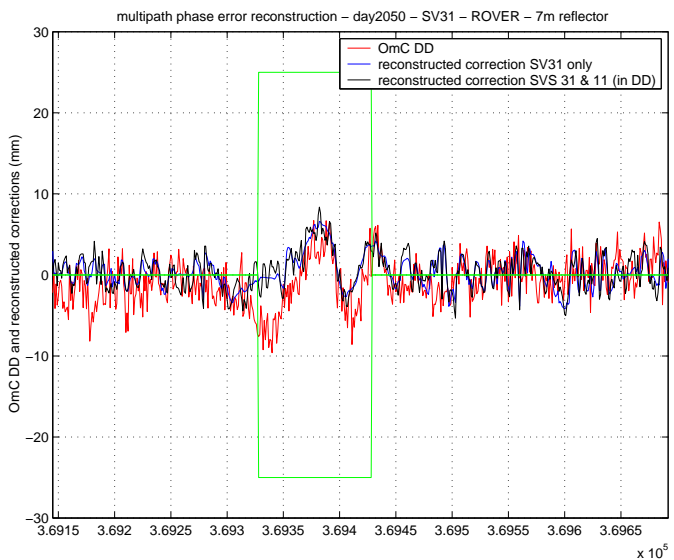

Figure 7.18c: reconstructed multipath error $(7 \mathrm{~m})$ 


\begin{tabular}{|c|c|c|c|c|c|c|}
\hline \multicolumn{2}{|c|}{ sv dura } & \multirow{2}{*}{$\begin{array}{l}\mathrm{sd} \\
8.0\end{array}$} & \multicolumn{2}{|c|}{ sdx gain\% } & \multicolumn{2}{|c|}{ sdn gaino } \\
\hline 1 & 306 & & 8.6 & -6.8 & 8.2 & -1.9 \\
\hline 3 & 460 & 10.1 & 8.0 & 20.8 & 9.3 & 8.4 \\
\hline 4 & 103 & 4.2 & 3.7 & 11.3 & 3.2 & 23.4 \\
\hline 7 & 614 & 8.4 & 7.9 & 5.3 & 8.8 & -5.0 \\
\hline 8 & 400 & 11.8 & 10.7 & 9.6 & 11.2 & 5.4 \\
\hline 9 & 313 & 5.5 & 6.0 & -9.4 & 7.0 & -27.4 \\
\hline 10 & 290 & 5.0 & 5.2 & -3.5 & 4.8 & 5.5 \\
\hline 11 & 439 & 10.8 & 10.1 & 6.1 & 10.6 & 2.2 \\
\hline 13 & 514 & 6.2 & 5.9 & 5.1 & 5.6 & 10.1 \\
\hline 20 & 412 & 11.6 & 11.8 & -2.1 & 11.2 & 3.5 \\
\hline 24 & 187 & 9.9 & 10.1 & -1.9 & 10.2 & -2.4 \\
\hline 27 & 507 & 8.6 & 7.8 & 9.3 & 8.2 & 5.4 \\
\hline 28 & 201 & 4.1 & 3.3 & 19.8 & 4.0 & 1.1 \\
\hline 31 & 305 & 4.6 & 4.1 & 11.8 & 3.8 & 17.4 \\
\hline & $0 ל 1$ & 9.2 & 8.7 & 6.0 & 9.1 & 1.3 \\
\hline
\end{tabular}

Table 7.5a: statistical results of the reconstruction for day203 (panel at $1 \mathrm{~m}$ )

Note: the same table (table 7.5a*) has been computed again after removing the data corresponding to low elevated satellites ( $15^{\circ}$ threshold) that are included in the multipath window time zones. Such data also exist for the test at $4 \mathrm{~m}$ (SV28 only, see table 7.5b*) but do not exist for the test at $7 \mathrm{~m}$.

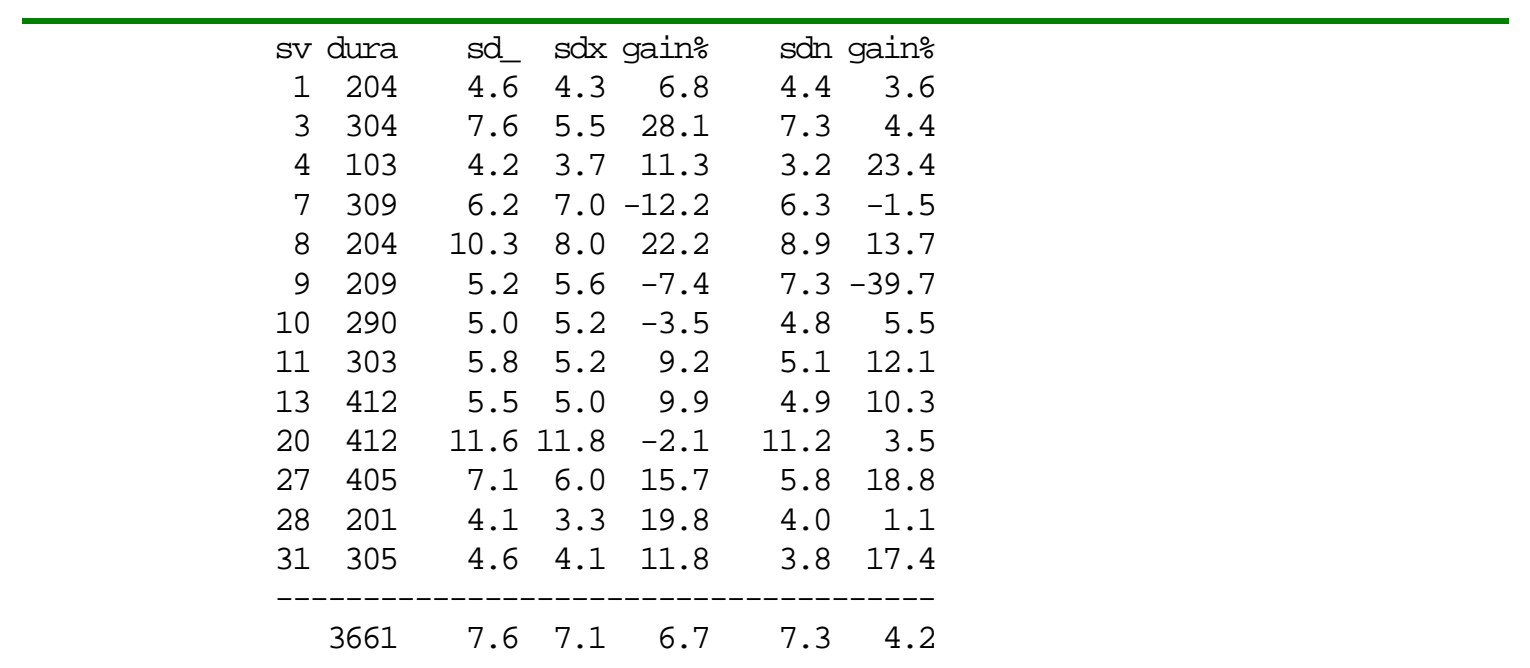

Table $7.5 a^{*}$ : same as table $7.5 \mathrm{a}$, but SVS with elevation $>15^{\circ}$ only are included 


\begin{tabular}{|c|c|c|c|c|c|}
\hline \multicolumn{2}{|c|}{ sv dura } & \multirow{2}{*}{$\begin{array}{l}\mathrm{sd} \\
3.4\end{array}$} & \multicolumn{2}{|c|}{ sdx gain\% } & sdn gain\% \\
\hline 1 & 102 & & 2.9 & 15.5 & $2.8 \quad 15.6$ \\
\hline 3 & 200 & 4.6 & 2.9 & 37.1 & $3.5 \quad 22.9$ \\
\hline 4 & 409 & 4.9 & 3.8 & 23.4 & $4.1 \quad 15.9$ \\
\hline 7 & 210 & 5.3 & 4.9 & 7.5 & 5.2 \\
\hline 8 & 371 & 5.4 & 4.0 & 26.3 & $4.3 \quad 21.3$ \\
\hline 9 & 109 & 5.9 & 6.1 & -2.7 & $8.7-46.8$ \\
\hline 10 & 306 & 5.4 & 3.9 & 27.7 & 4.124 .3 \\
\hline 11 & 198 & 4.9 & 4.2 & 13.0 & $5.0-1.6$ \\
\hline 13 & 206 & 5.0 & 4.0 & 19.6 & $4.3 \quad 12.5$ \\
\hline 17 & 107 & 9.2 & 9.4 & -1.8 & $14.0-51.7$ \\
\hline 20 & 314 & 6.3 & 5.1 & 18.3 & $5.3 \quad 15.0$ \\
\hline 24 & 104 & 4.1 & 4.3 & -5.0 & $3.2 \quad 22.6$ \\
\hline 27 & 339 & 5.6 & 4.0 & 28.4 & $4.5 \quad 18.3$ \\
\hline 28 & 99 & 5.0 & 3.7 & 26.4 & $3.7 \quad 24.7$ \\
\hline 29 & 228 & 7.1 & 7.7 & -7.7 & $8.9-24.4$ \\
\hline 31 & 303 & 4.9 & 3.6 & 26.6 & 4.5 \\
\hline & 3605 & 5.9 & 5.0 & 14.3 & 5.8 \\
\hline
\end{tabular}

Table 7.5b: statistical results of the reconstruction for day204 (panel at $4 \mathrm{~m}$ )

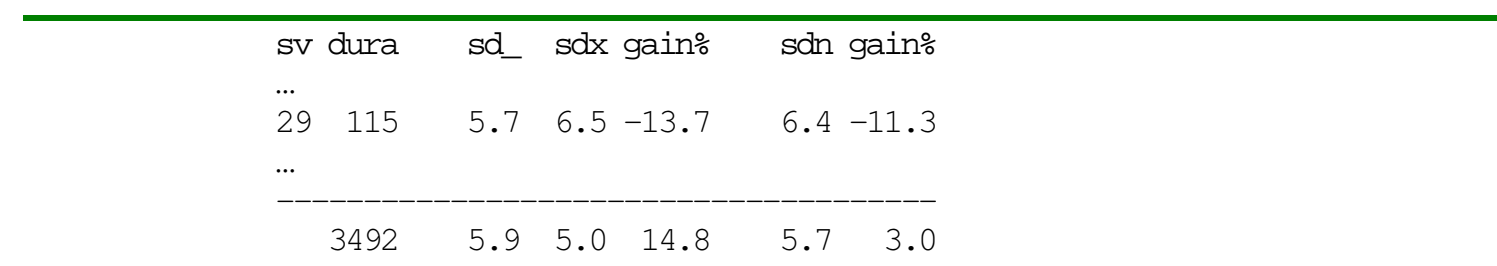

Table $7.5 b^{*}$ : same as table $7.5 b$, but svs with elevation $>15^{\circ}$ only are included

\begin{tabular}{|c|c|c|c|c|c|c|}
\hline SV & dura & sd_ & $s d x$ & gain。 & sdn & gaino \\
\hline 1 & 160 & 3.7 & 2.5 & 32.5 & 2.6 & 30.9 \\
\hline 3 & 200 & 4.2 & 2.8 & 33.6 & 3.7 & 13.0 \\
\hline 4 & 204 & 4.5 & 2.8 & 37.2 & 3.1 & 30.6 \\
\hline 7 & 208 & 4.4 & 3.4 & 23.5 & 3.9 & 10.6 \\
\hline 8 & 204 & 3.6 & 3.0 & 16.3 & 3.2 & 9.1 \\
\hline 10 & 204 & 4.6 & 2.6 & 43.6 & 2.9 & 35.3 \\
\hline 11 & 100 & 4.4 & 3.1 & 29.2 & 3.0 & 32.7 \\
\hline 13 & 103 & 4.4 & 2.5 & 42.3 & 4.1 & 7.2 \\
\hline 17 & 1 & 0.0 & 0.0 & $\mathrm{NaN}$ & 0.0 & $\mathrm{NaN}$ \\
\hline 20 & 193 & 3.7 & 2.4 & 35.2 & 3.5 & 5.2 \\
\hline 24 & 103 & 6.2 & 3.6 & 42.3 & 4.8 & 23.5 \\
\hline 27 & 202 & 3.2 & 2.6 & 20.3 & 3.6 & -11.4 \\
\hline 29 & 8 & 2.1 & 2.2 & -5.6 & 2.0 & 5.8 \\
\hline 31 & 202 & 3.9 & 2.6 & 33.5 & 3.0 & 22.2 \\
\hline & 92 & 4.7 & 3.3 & 29.3 & 4.4 & 6.9 \\
\hline
\end{tabular}

Table 7.5c: statistical results of the reconstruction for day205 (panel at $7 \mathrm{~m}$ ) 
The results of the reconstruction process in kinematic mode are summarised in table 7.6 and displayed in bold, for all satellites and all multipath zones. The preceding results (see Chapter 6, table 6.7) corresponding to the standard measurements and those modified by the PMMW corrections are given for memory.

Similarly as in static mode, one can also compare the data output from the reconstruction process to the standard and PMMW corrected data, for all satellites and all epochs, irrespective of the multipath zones. This is given in table 7.7.

\begin{tabular}{clll}
\hline Kinematic tests & $\sigma$ & $\sigma_{\mathrm{PMMN}}$ & Gain \\
\hline $\begin{array}{c}\text { reflector } 1 \mathrm{~m} \\
0.7 \mathrm{~m}<\text { addpath }<2.0 \mathrm{~m}\end{array}$ & $9.2 \mathrm{~mm}$ & $8.7 \mathrm{~mm}$ & $6 \%$ \\
$\begin{array}{c}\text { reconstruction } \\
\text { reflector } 1 \mathrm{~m} \\
\text { elevation of satellites }>15^{\circ}\end{array}$ & $7.6 \mathrm{~mm}$ & $7.1 \mathrm{~mm}$ & $7 \%$ \\
\hline $\begin{array}{c}\text { reflector } 4 \mathrm{~m} \\
3.0 \mathrm{~m}<\text { addpath }<7.6 \mathrm{~m}\end{array}$ & $5.9 \mathrm{~mm}$ & $5.0 \mathrm{~mm}$ & $14 \%$ \\
$\begin{array}{c}\text { reflector } 4 \mathrm{~m} \\
\text { elevation of satellites }>15^{\circ}\end{array}$ & $5.9 \mathrm{~mm}$ & $5.8 \mathrm{~mm}$ & $1 \%$ \\
\hline $\begin{array}{c}\text { reflector } 7 \mathrm{~m} \\
5.1 \mathrm{~m}<\text { addpath }<11.9 \mathrm{~m}\end{array}$ & & $5.7 \mathrm{~mm}$ & $15 \%$ \\
\hline
\end{tabular}

Table 7.6: L1 phase DD statistics in kinematic mode (for "standard", "PWWM corrected" and "reconstructed" phase data) 


\begin{tabular}{llll}
\hline Kinematic tests & $\sigma$ & $\sigma_{\mathrm{PMMN}}$ & $\sigma_{\text {reconstruction }}$ \\
\hline reflector $1 \mathrm{~m}$ & $8.1 \mathrm{~mm}$ & $8.1 \mathrm{~mm}$ & $8.7 \mathrm{~mm}$ \\
elevation of satellites $>15^{\circ}$ & $6.5 \mathrm{~mm}$ & $6.5 \mathrm{~mm}$ & $7.1 \mathrm{~mm}$ \\
reflector $4 \mathrm{~m}$ & $8.2 \mathrm{~mm}$ & $8.1 \mathrm{~mm}$ & $8.7 \mathrm{~mm}$ \\
elevation of satellites $>15^{\circ}$ & $6.6 \mathrm{~mm}$ & $6.5 \mathrm{~mm}$ & $6.9 \mathrm{~mm}$ \\
reflector $7 \mathrm{~m}$ & $6.7 \mathrm{~mm}$ & $6.6 \mathrm{~mm}$ & $7.2 \mathrm{~mm}$ \\
elevation of satellites $>15^{\circ}$ & $4.0 \mathrm{~mm}$ & $3.8 \mathrm{~mm}$ & $4.5 \mathrm{~mm}$ \\
\hline
\end{tabular}

Table 7.7: L1 phase DD statistics in kinematic mode (all data, all epochs) (for "standard", "PWWM corrected" and "reconstructed" phase data)

In the case of kinematic tests, the algorithm is capable of doing the worst as well as the best (see table 7.5a, 7.5b and 7.5c where large differences of results between satellites are shown).

It is clear that the multipath phenomena is weaker in kinematic mode than in static mode, at least with the conditions of these tests. Data contain information that tends to be buried in noise.

It might also be possible that there exists a certain coincidence of the drawback of the PMMW measurement and that of the reconstruction process, since this uses PMMW amongst other observables. Or in other words, it sometimes happens that the reconstruction process fails when the PMMW estimate of the multipath phase error is degraded (because the additional path length of the reflected signal L gets significantly under the $7.5 \mathrm{~m}$ threshold). This is illustrated in the example given in Fig. 7.19 and Fig. 7.20 (with SV17, and SV29 as the differencing satellite). Table 6.6b in Chapter 6 gives that $\mathrm{L}$ is comprised between 3.4 and $3.7 \mathrm{~m}$ for SV17, which is small. 


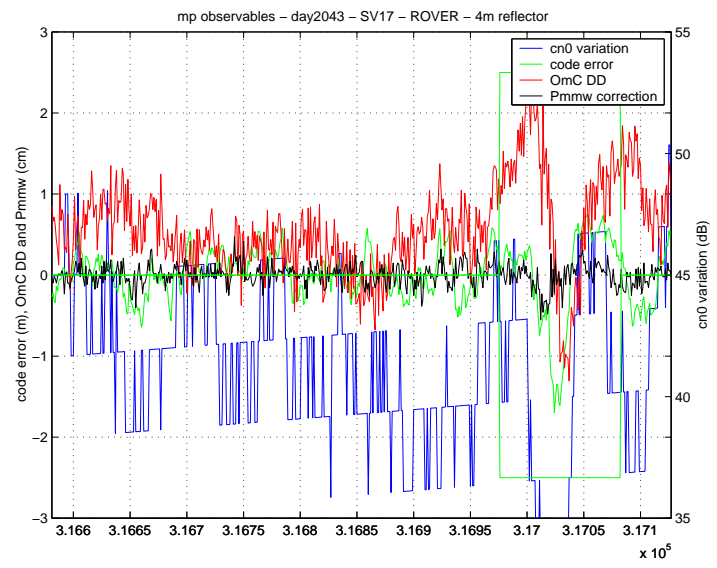

Figure 7.19: observables used in the reconstruction process for SV17 (4 $\mathrm{m}$ test)
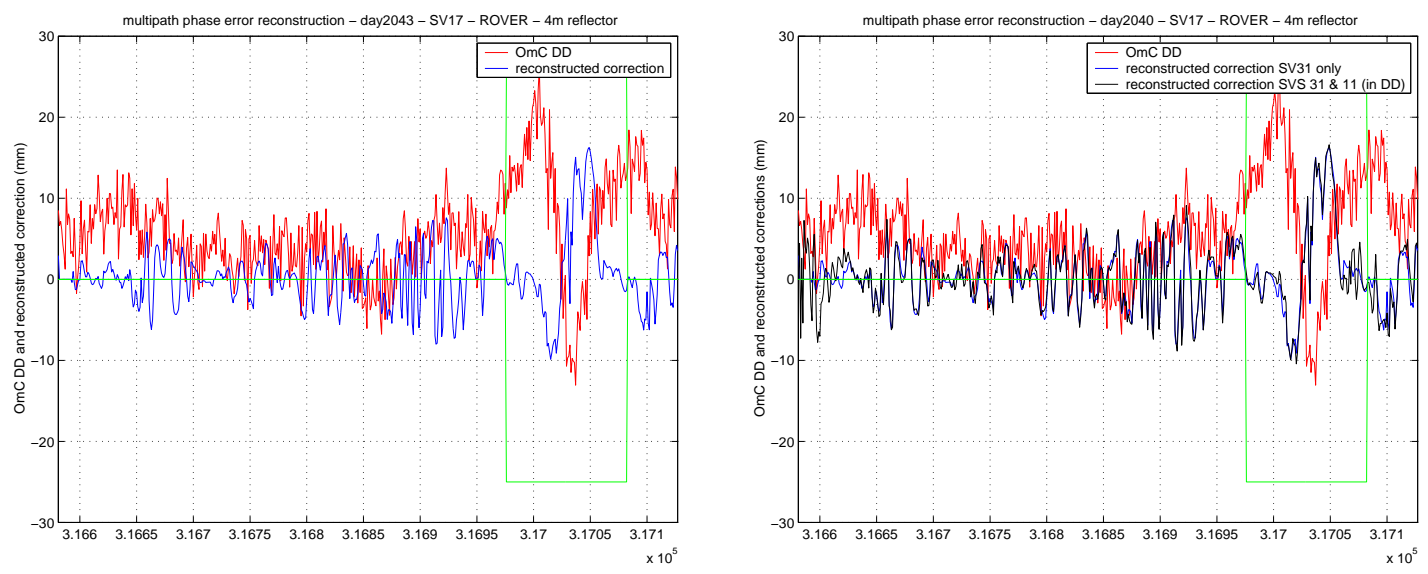

Figure 7.20: reconstructed multipath phase error for SV17 (left figure) and combination with that for SV29 in a global correction of the O-C DD of phase (4 m test)

In this example, the phase of the reconstructed error is driven by the phase of the PMMW correction that is abnormally shifted from the phase of the actual multipath phase error (i.e. the phase of the O-C DD of phase L1 in Fig. 7.19).

\section{5 - Conclusions about the reconstruction processes}

Several investigations have been carried out in order to overcome the key limitation of the PMMW technique, i.e. the degradation of its efficiency as the additional path length decreases. 
The new contribution of this chapter is a consideration of the combination of the PMMW measurements with two other phase multipath observables: signal-to-noise ratio and code multipath error. It has been demonstrated that:

- the combination of PMMW and SNR is an efficient method for modelling phase multipath error resulting from reflectors more than a few metres away from the antenna. Essentially, the PMMW indicates the phase of the multipath error (without sign ambiguity) and the SNR its amplitude.

- For close reflectors the multipath code error can be used instead of the PMMW to indicate the phase of the correction, because the multipath code error shifts in phase for additional distance under a few metres. This property of the code might result from the implementation of the code correlator and might be true only for System 500 receivers. Further investigations are needed.

An intelligent adaptive algorithm that can combine all three multipath observables (PMMW, SNR and code multipath error) in order to estimate phase multipath errors, whatever the distance is to the reflector, has been designed.

In static mode, a real improvement in the Observed-Computed double difference of L1 phase is obtained in the case of a very close reflector (around $10 \%$ improvement of the O-C DD of L1 phase with respect to the standard phase data, versus only a few percent carried out by the PMMW phase correction). But this algorithm seems to make a $20 \%$ improvement at the most, whereas the PMMW phase correction reaches $50 \%$ when the reflector gets further away (over a few metres).

In kinematic mode, experimental conditions and data that has been collected so far do not validate the efficacy of the process in kinematic mode. It actually seems capable of worsening as well as improving kinematic data sets. A key factor to explain this drawback is the quality of the observables: their magnitude is reduced and they are more or less buried in noise, and their fidelity to the modelling does not seem to be sufficient. 
Chapter 8

\section{CONCLUSIONS AND SUGGESTIONS FOR FURTHER WORK}

\section{$8.1-$ Conclusions}

- After a simplified description of the physics of the multipath phenomenon (Chapter 1) and a review of various multipath mitigation techniques collected in the literature (Chapter 2), this research has focused on multipath functional modelling, with a detailed presentation of standard GPS receiver loops (Chapter 3) and how these loops deliver measurements with certain multipath typical errors (Chapter 4). For this modelling, the hypotheses necessary for considering multipath as specular reflections were made, i.e. a sufficiently large and smooth reflecting surface. Whereas most papers in the literature present the multipath phase error through a phasor representation only, this thesis has tried to start from the discrimination function of the phase loop, and similarly for the multipath code error, which rigorously shows the dependency between these errors.

- In Chapter 5, this thesis gives a thorough analysis of the functioning of the code and phase multipath mitigation window correlators, patented by Leica. These window correlators are basically original sampling techniques that, for code tracking, result in a strictly and remarkably reduced multipath error envelope ( $2 \mathrm{~m}$ maximum and null above a code delay of $10 \mathrm{~m}$ ) and, for phase tracking, provide an estimation of the multipath phase error due to the standard phase loop. Both code and phase MMW have been implemented in the ASIC of System 500 receivers for a few years (with C/A-code and L1 carrier), but only the code MMW was used in the customer release. Until now, phase data was still output from a standard correlator, and not corrected by the phase MMW estimation. 
- Part of the work programmed in this research was to assess the performance of the Phase Multipath Mitigation Window technique (PMMW). This is reported in Chapter 6.

The static and kinematic tests that were carried out in this programme of research proved that the measurement provided by the PMMW technique improves the precision of the L1 phase data by up to $50 \%$ (with lightweight antennas and in Double Differences on a baseline of $100 \mathrm{~m}$ ), without any significant undesirable effect. Hence, it appears as the main conclusion of these tests, that the PMMW technique should definitely be used to correct the standard phase data in a future version of receivers.

It is important that the use of the SESSYL facility and the design of an original tests methodology be underlined in this conclusion. No previous work concerned by the accuracy of kinematic GPS was done in which a rover was effectively moved on a reference trajectory known with an accuracy of the order of the millimetre. This was provided by SESSYL that enabled Observed - Computed DD of phase to be computed in an effective kinematic environment. Multipath on phase data, as well as improvement due to the application of mitigation techniques on these data, were clearly shown by this way, even when the rover was moving.

The expected limitation of window correlators when multipath is due to a very close reflector (i.e. making an additional travelled path length of only a few metres or less) has been observed during the tests performed. The efficacy of the phase error estimation by the PMMW correlator (and the consecutive phase correction) progressively reduces, from $50 \%$ down to only a few percent, when the code delay of the reflected signal gets under the $25 \mathrm{~ns}$ receiver sampling period (i.e. approximately $7.5 \mathrm{~m}$ in terms of distance).

The comparison between the observed multipath phase error and that predicted by modelling has been studied. The necessity of using the antenna gain patterns in both right and left polarisations has been shown, and it has also been emphasized that the azimuth of the satellite with respect to the antenna is a key parameter in predicting the level of the phase multipath error. 
- Chapter 7, with investigations based on the GPS observables, typically illustrates multipath mitigation by functional modelling applied to the phase measurements.

An algorithm has been designed with the objective of overcoming the limitation of the PMMW technique for very close multipath. It is based on the use of the code and PMMW measurements, as well as the signal-to-noise ratio, in a multipath phase error reconstruction process. It can work in real-time, with a certain delay due to the necessity of computing the multipath code error through a dual frequency combination in a moving window.

An innovation is carried out by the fact that this algorithm takes advantage of observables other than the signal-to-noise ratio only. Particularly, the direct estimation of the multipath phase error (provided for each tracked satellite by the corresponding PMMW correlator) is used. This estimation gives the correction to be applied to phase data with directly the right sign, whereas this used to be ambiguous when reconstructed by SNR only. The algorithm also takes advantage of the multipath code error, for which it has been found an unexpected (and very useful) behaviour in the case of a close reflector. For this case, the theoretical modelling of the multipath code error has been modified, in order to fit with the experiment results.

In a multipath environment with additional path lengths of up to about 2 metres, and in static mode, the global improvement of L1 phase data (in DD) due to the new algorithm is of the order of $10 \%$ in average. In the same conditions, but in kinematic mode, the results are still rather uneven. Moreover, the new algorithm is still unable to reach the level of mitigation of the PMMW correlator in the case of a more distant reflector. These results are due to both the actual tuning of the process (that does not sufficiently filter the ambient noise) and also possible drawback in modelling the observables.

Lastly, it should be noted that the reconstruction process was run twice: first with a $1 \mathrm{~dB}$ binned signal-to-noise ratio and second with its full resolution. Both give very similar results. A suggestion in [BARNES, 2000] was to use a better defined SNR data in the ANF/ALS filtering algorithm: this seems in fact not to improve results, and let us conclude that a $1 \mathrm{~dB}$ resolution is sufficient in such a process. 


\section{2 - Suggestions for further work}

As far as the PMMW technique is concerned, no specific test with multiple reflectors was done in the frame of this thesis. By theory, multiple reflections are expected to be mitigated simultaneously. The tests performed by Leica on its rooftop in static mode probably give a first answer to this question, but it would be interesting to confirm this ability of the technique also in kinematic mode, and in a controlled multipath environment.

About the functional modelling and the multipath phase error reconstruction process, a better tuning of the ANF/ALS is worth to be investigated, in order to attenuate its sensitivity to ambient noise on phase data. Of course, a balance should be found with its ability to detect multipath. Other adaptive spectral analysers could be tested on the data sets, and implemented in new versions of the reconstruction algorithm, for comparison purpose.

But it is known that the improvement is theoretically limited. Actually, if one uses two signals in quadrature (like PMMW correction and SNR variation in the context of GPS multipath), any linear process that combines these signals, with the objective of either reducing the error level or improving the signal-to-noise ratio, brings about a reduction/improvement of $50 \%$ maximum. Moreover, this maximum $50 \%$ gain could be reached only if modelling and observations input in the reconstruction process were perfect!

Hence, it seems that further investigations should also concentrate on the quality of the modelling of the observables. In this work for instance, it has been noted (and effectively used) an unexpected and initially non-modelled behaviour of the multipath code error, for a reason that remains uncertain, but that might relate to the receiver implementation design. Simulation of the receiver functioning has not been very advanced in this research, and maybe this could bring about interesting ideas, particularly in the case of multipath with a code delay that is below the sampling period of the ASIC. 
Moreover, new tests with other receivers of the same class (receivers that share the same reference waveform correlation technology) might lead to interesting observations and possible explanations of the behaviour of the multipath code error for very close reflectors.

The adaptive algorithm that this thesis suggests is a first step to be further improved. It seems that the process might be made more intelligent by optimising the manner in which it is applied with that of the direct PMMW corrections.

More generally.

- The use of the PMMW corrections in the design of a new stochastic modelling of the phase measurements would appear to be a fruitful avenue for future research.

- The variety of the observables is also worth to be deepen (like using additional carrier frequencies or P-code). New techniques have been presented recently (like [WEILL, 2003]) that go in this direction.

- It should be underlined that, whereas most references in the literature dealing with multipath error reconstruction algorithm were studied on limited data sets, this work extends the process to a quite large GPS data collection. The tests methodology as well as the data already collected are also of great interest to assess the performances of mitigation techniques that are still under development.

- And lastly, this work tries to meet real-time specification requirements, typically in filtering design. If the constraint of real-time was suppressed, then a wide variety of smoothing techniques would be allowed as well as reverse processing of the data. For a number of applications, like precise road 3-dimensional levelling, post-processing of data is clearly possible, and real-time not mandatory. Future work connected with the applications of precise GPS positioning might actually extend the process and probably obtain better results. 


\section{REFERENCES}

[BARNES, 2000] - Barnes, J.B., Real Time Kinematic GPS and multipath: characterisation and improved least squares modelling, $\mathrm{PhD}$ thesis, University of Newcastle, 2000

[BÉTAILLE et al., 2000] - Bétaille, D., C. Lemaire, F. Peyret, J.M. Prual, Notice SESSYL, Laboratoire Central des Ponts et Chaussées, Nantes, 2000

[BÉTAILLE, 2003] - Bétaille, D., A testing methodology for GPS phase multipath mitigation techniques, Proceedings of ION GPS-03, The Institute of Navigation, Portland, Oregon, 2003

[BÉTAILLE et al., 2003-1] - Bétaille, D., J. Maenpa, H.J. Euler, P. Cross, A new approach to GPS phase multipath mitigation, Proceedings of ION NTM-03, The Institute of Navigation, Anaheim, California, 2003

[BÉTAILLE et al., 2003-2] - Bétaille, D., J. Maenpa, H.J. Euler, P. Cross, Overcoming the limitations of the phase multipath mitigation window, Proceedings of ION GPS-03, The Institute of Navigation, Portland, Oregon, 2003

[BISNATH et al., 1997] - Bisnath, S.B., J.P. Collins, R.B. Langley, GPS multipath assessment of the Hilbernia oil platform, a study commissioned by Cougar Helicopters Ltd., Geodetic Research Laboratory, University of New Brunswick, 1997

[BRAASCH, 1996] - Braasch, MS., Multipath effects, chapter 14 in Global Positioning System: theory and applications, edited by B.W. Parkinson and J.J. Spilker, Progress in Astronautics and Aeronautics Series, AIAA, 1996. 
[BRODIN, 2001] - Brodin, G., Review and selection of most suitable carrier multipath mitigation techniques for GPS attitude determination for small spacecraft, a study prepared for DERA, Space Department, University of Leeds, March 2001

[BRUNNER et al., 1999] - Brunner, F.K., H. Hartinger, L. Troyer, GPS signal diffraction modelling: the stochastic SIGMA- $\Delta$ model, Journal of Geodesy, Vol. 73, pp. 259-267, 1999

[CHAGGARA et al., 2002] - Chagarra, R., C. Macabiau, E. Chatre, Using GPS multicorrelator receivers for multipath parameters estimation, Proceedings of ION GPS02, The Institute of Navigation, Portland, Oregon, 2002

[COMP and AXELRAD, 1996] - Comp, C.J., P. Axelrad, An adaptive SNR-based carrier phase multipath mitigation technique, Proceedings of ION GPS-96, pp. 683-697, The Institute of Navigation, Kansas City, Missouri, 1996

[DURAND, 2003] - Durand, S., Amélioration de la précision de la localisation différentielle temps réel par mesure de phase des systèmes GNSS: étude détaillée des équations d'observation et du problème de résolution des ambiguittés entières, thèse de Doctorat, Observatoire de Paris, 2003

[EL-RABBANY, 1995] - El-Rabbany, A., Temporal characteristics of multipath errors, Proceedings of ION GPS-95, pp. 1493-1497, The Institute of Navigation, Palm Springs, California, 1995

[FARRET and SANTOS, 2001] - Farret, J.C., M.C. Santos, An alternative method for detection and mitigation of static multipath in L1 carrier phase measurements, Proceedings of ION NTM-01, The Institute of Navigation, Long Beach, California, 2001

[FILIPPOV et al., 1999] - Filippov, V., D. Tatarnicov, J. Ashjaee, A. Astakhov, I. Sutiagin, The first dual-depth dual-frequency choke ring, Proceedings of ION GPS-99, pp. 1035-1039, The Institute of Navigation, Salt Lake City, Utah, 1999 
[GARIN and ROUSSEAU, 1996] - Garin, L., J. Rousseau, Enhanced strobe correlator multipath rejection for code and carrier, Proceedings of ION GPS-97, pp. 559-568, The Institute of Navigation, Kansas City, 1999

[GEORGIADOU and KLEUSBERG, 1987] - Georgiadou, Y., A. Kleusberg, On carrier signal multipath effects in relative GPS positioning, Manuscripta Geodaetica, Vol. 13, pp. 172-179, 1988

[GREWAL et al., 2001] - Grewel, M.S., L.R. Weill, A.P. Andrews, Global positioning systems, inertial navigation and integration, published by Wiley \& Sons Inc., 2001

[HANDEL and TICHAVSKY, 1994] - Handel, P., P. Tichavsky, Adaptive estimation for periodic signal enhancement and tracking, International Journal of Adaptive Control and Signal Processing, 9, pp. 447-456, 1994

[HANNAH, 2001] - Hannah, B.M. Modelling and simulation of GPS multipath propagation, $\mathrm{PhD}$ thesis, Queensland University of Technology, 2001

[HATCH et al., 1997] - Hatch, R.R., R.G. Keegan, T.A. Stansell, Leica's code and phase multipath mitigation techniques, a Leica's letter, Torrance, California, 1997

[HATCH, 2000] - Hatch, R.R., Method and apparatus for code synchronisation in a GPS receiver, US patent 6,163,567, 2000

[KAPLAN et al., 1996] - Kaplan, E.D., J.L. Leva, M.S. Pavloff, Fundamentals of satellite navigation, in Understanding GPS principles and applications, edited by E.D. Kaplan and published by Artech House Inc., 1996

[KEE and PARKINSON, 1994] - Kee, C., B. Parkinson, Calibration of multipath errors on GPS pseudorange measurements, Proceedings of ION GPS-94, pp. 353-362, The Institute of Navigation, Salt Lake City, Utah, 1994

[LANGLEY, 1997] - Langley, R.B., GPS receiver system noise, Innovation in GPS World, Vol. 8, No. 6, June 1997, pp. 40-45 
[MADER, 1994] - Mader, G.L., GPS antenna calibration at the National Geodetic Survey, http://www.ngs.noaa.gov/ANTCAL/Files/summary.html, Silver Spring, MD, USA, 1994.

[MALICORNE, 2001] - Malicorne, M., Analyse des performances de systèmes de navigation par satellites pour les applications en environnement urbain, thèse de Doctorat, Ecole Nationale Supérieure de l’Aéronautique et de l'Espace, 2001

[McGRAW and BRAASCH, 1999] - McGraw, G., M. Braasch, GNSS multipath mitigation using gated correlator technique, Proceedings of ION NTM-99, The Institute of Navigation, San Diego, California, 1999

[NEHORAI, 1985] - Nehorai, A., A minimal parameter adative notch filter constrained poles and zeros, IEEE Transactions on Acoustics, Speech and Signal Processing, 33 (4), pp. 983-996, 1985

[RAY et al., 1999] - Ray, J.K., M.E. Cannon, P. Fenton, Code range and carrier phase multipath mitigation using SNR, range and phase measurements in a multi-antenna system, Proceedings of ION GPS-99, pp. 713-725, The Institute of Navigation, Nashville, Tennessee, 1999

[RAY, 1999] - Ray, J.K., Use of multiple antennas to mitigate carrier phase in reference stations, Proceedings of ION GPS-99, pp. 269-279, The Institute of Navigation, Nashville, Tennessee, 1999

[RAY, 2000] - Ray, J.K., Mitigation of GPS code and carrier phase multipath effects using a multi-antenna system, $\mathrm{PhD}$ thesis, University of Calgary, 2000

[RICHTER and EULER, 2001] - Richter, B., H.J. Euler, Study of improved observation modeling for surveying type applications in multipath environment, Proceedings of ION GPS-01, The Institute of Navigation, Salt Lake City, Utah, 2001 
[ROTHACHER et al, 1995] - Rothacher, M. S. Schaer, L. Mervart, G. Beutler, Determination of antenna phase center variation using GPS data, IGS Workshop, Potsdam, Germany, May 1995.

[SCHUPLER, 2001] - Schupler, B.R., T.A. Clark, Characterizing the behaviour of geodetic GPS antennas, Innovation in GPS World, Vol. 12, No. 2, February 2001, pp. 4855

[STANSELL et al., 1996] - Stansell, T.A., J.E. Knight, R.G. Keegan, R.R. Hatch, C.R. Cahn, Mitigation of multipath effects in GPS receivers, world patent WO 96/37789, 1996

[STANSELL et al., 2000] - Stansell, T.A., J.E. Knight, R.G. Keegan, C.R. Cahn, Mitigation of multipath effects in GPS receivers, US patent 6,160,841, 2000

[STANSELL and MAENPA, 1999] - Stansell, T.A., J. Maenpa, ClearTrak GPS receiver technology, a Leica's letter, Torrance, California, 1999

[TOWNSEND and FENTON, 1994] - Townsend, B., P. Fenton, A practical approach of pseudorange multipath errors in a L1 GPS receiver, Proceedings of ION GPS-94, The Institute of Navigation, Salt Lake City, Utah, 1994

[TOWNSEND et al, 1995] - Tounsend, B., P. Fenton, K. Van Dierendonck, R.D.J. Van Nee, L1 carrier phase multipath error reduction using MEDLL technology, Proceedings of ION GPS-95, The Institute of Navigation, Palm Springs, California, 1995

[VAN NEE, 1992] - Van Nee, R.D.J., The Multipath Estimating Delay Lock Loop (MEDLL), IEEE Second International Symposium on Spread Spectrum Techniques and Applications, Yokohama, Japan, 1992

[VAN NEE, 1995] - Van Nee, R.D.J., Multipath and multi-transmitter interference in spread-spectrum communication and navigation systems, $\mathrm{PhD}$ thesis, University of Delft, 1995 
[WARD, 1996] - Ward, P., GPS satellite signal characteristics, acquisition and tracking, in Understanding GPS principles and applications, edited by E.D. Kaplan and published by Artech House Inc., 1996

[WEILL, 1997] - Weill, L.R., Conquering multipath: the GPS accuracy battle, Innovation in GPS World, Vol. 8, No. 4, April 1997, pp. 59-66

[WEILL, 2003] - Weill, L.R., High-performance multipath mitigation using the synergy of composite GPS signals, Proceedings of ION GPS-03, The Institute of Navigation, Portland, Oregon, 2003

[WIESER and BRUNNER, 1999] - Wieser, A., F.K. Brunner, An extended weight model for GPS phase observations, Earth Planets Space, Vol. 52, pp. 777-782, 2000

[YOUNG et al., 1985] - Young, L.E., R.E. Neilan, and F.R. Bletzacker, GPS satellite multipath: an experimental investigation, Proceedings of GPS Positioning Symposium, pp. 423-432, Rockville, MD, 1985. 
APPENDIX 1: OPTIMISATION OF THE SHAPE OF THE PHASE MMW

Here are displayed, for a 15 chips long PRN code, different MMWs and their correlation with punctual and delayed codes (until a delay of 1 chip). See also Fig. 5.22.

Symmetric MMW at code transitions

$\mathrm{C}(\mathrm{t})$

phase MMW

$\mathrm{C}(\mathrm{t}) *$ phase MMW

$\mathrm{C}(\mathrm{t}-\mathrm{d})$

$\mathrm{C}(\mathrm{t}-\mathrm{d}) *$ phase MMW

$\mathrm{C}(\mathrm{t}-1$ chip $)$

$\mathrm{C}(\mathrm{t}-1$ chip)*phase MMW

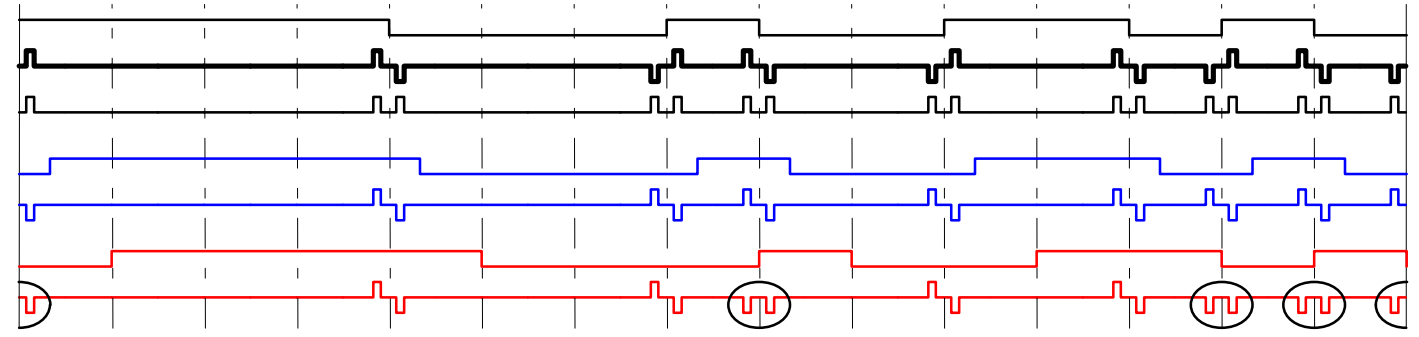

Symmetric MMW at every code clock

$\mathrm{C}(\mathrm{t})$

phase MMW

$\mathrm{C}(\mathrm{t}) *$ phase MMW

$\mathrm{C}(\mathrm{t}-\mathrm{d})$

$\mathrm{C}(\mathrm{t}-\mathrm{d}) *$ phase MMW

$\mathrm{C}(\mathrm{t}-1$ chip $)$

$\mathrm{C}(\mathrm{t}-1 \text { chip })^{*}$ phase MMW

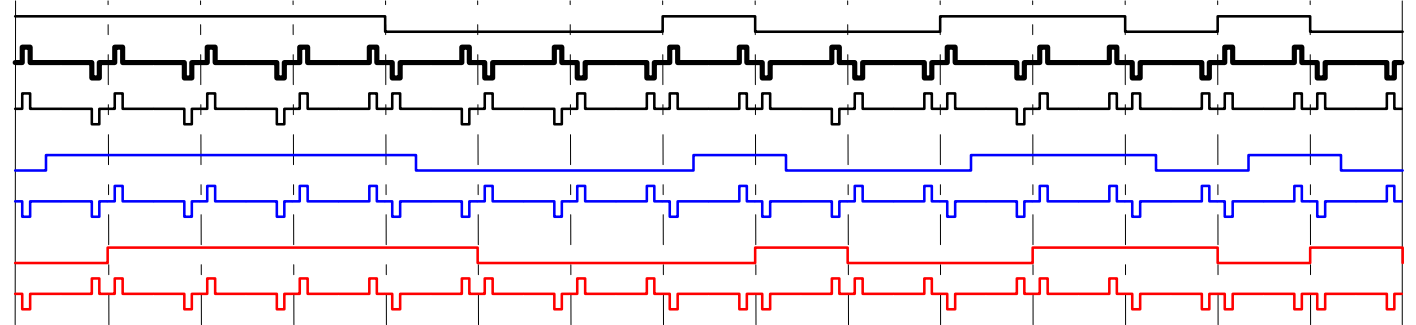

Asymmetric MMW at code transitions

$\mathrm{C}(\mathrm{t})$

phase MMW

$\mathrm{C}(\mathrm{t})$ *phase MMW

$\mathrm{C}(\mathrm{t}-\mathrm{d})$

$\mathrm{C}(\mathrm{t}-\mathrm{d}){ }^{*}$ phase MMW

$\mathrm{C}(\mathrm{t}-1$ chip $)$

$\mathrm{C}(\mathrm{t}-1$ chip)*phase MMW

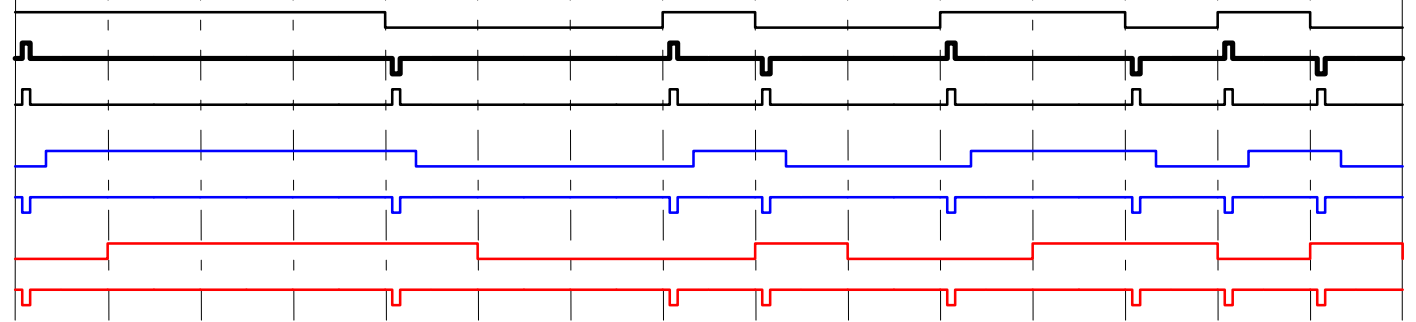

Asymmetric MMW at every code clock

$\mathrm{C}(\mathrm{t})$

phase MMW

$\mathrm{C}(\mathrm{t}) *$ phase MMW

$\mathrm{C}(\mathrm{t}-\mathrm{d})$

$\mathrm{C}(\mathrm{t}-\mathrm{d}) *$ phase MMW

$\mathrm{C}(\mathrm{t}-1$ chip $)$

$\mathrm{C}(\mathrm{t}-1 \text { chip })^{*}$ phase MMW

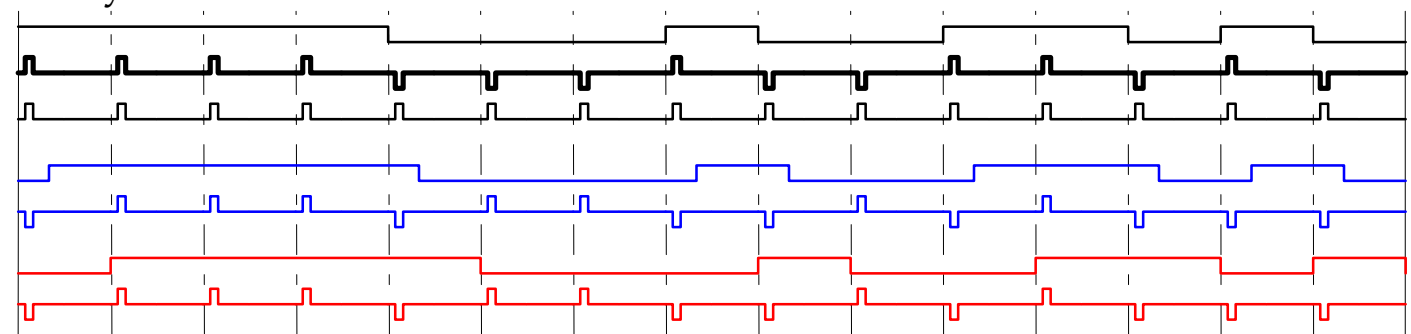



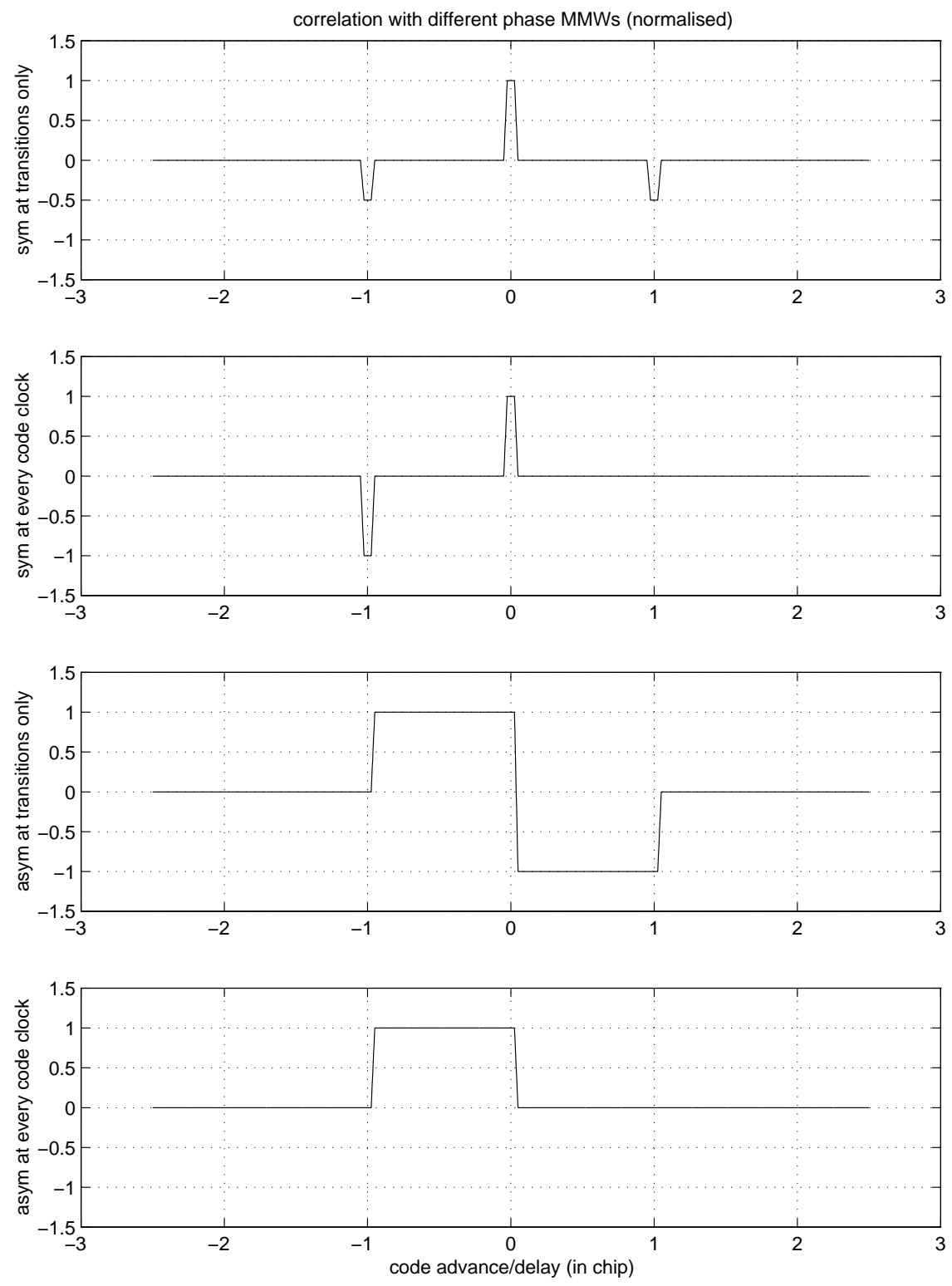

Contrary to code discrimination functions, MMWs correlation outputs should not be zero for punctual code. However, if the code is advanced/delayed, the MMWs correlation should output zero, as soon as the advance/delay exceeds a certain critical value. The main ideas to bear in mind are: $1^{\text {st }}$, MMWs repeated at every code clock solve the problem of correlation around 1 chip delay; $2^{\text {nd }}$, the signal-to-noise ratio is better with asymmetric than symmetric MMWs (useless components - in terms of signal content - are suppressed). 


\section{APPENDIX 2: ELECTROMAGNETIC MODELLING}

This appendix gives a computation of the coefficient of reflection, assuming that the reflection is fully described by the geometric optics (Snell-Descartes law of propagation). Transverse electromagnetic (TEM) waves are considered here, i.e. waves whose electric field $(\mathrm{E})$ is perpendicular to the direction of propagation (unit vector $\mathrm{X}$ ). The magnetic field $(\mathrm{H})$ is such that $\mathrm{XEH}$ is a direct frame. The electric field $\mathrm{E}$ of elliptically polarised waves have the following general form:

$\mathrm{E}=\mathrm{Ey}+\mathrm{Ez}$

where

$\mathrm{Ey}=\mathrm{E} \mathrm{y}_{\max } \sin (\mathrm{wt}-\mathrm{kx}) \mathrm{Y}$

$\mathrm{Ez}=\mathrm{Ez} \mathrm{max}_{\max } \sin (\mathrm{wt}-\mathrm{kx}-\delta) \mathrm{Z}$

and $Y$ and $Z$ are unit vectors that, together with $X$, generate a direct frame $X Y Z$.

These equations means that, in a given location (e.g. at the centre of an antenna), the electric field is rotating in time, and the extremity of vector $\mathrm{E}$ describes an ellipse.

Several particular cases are noticeable:

if $\delta=0$ or $180^{\circ}$, the polarisation is linear (the electric field is not rotating);

if $\delta=-90$ or $90^{\circ}$, the main axes of the ellipse are $x$ and $y$,

if $\mathrm{Ey}_{\max }=\mathrm{Ez}_{\max }$ and $\delta=-90^{\circ}$ or $90^{\circ}$, the polarisation is circular (the extremity of vector $\mathrm{E}$ describes a circle);

when: $-180<\delta<0$, the polarisation is right-hand (RHEP, or RHCP if $\delta=-90^{\circ}$ );

when: $0<\delta<180$, the polarisation is left-hand (LHEP, or LHCP if $\delta=90^{\circ}$ ). 
The following figures illustrate different elliptical polarisations, including the particular circular (RHCP and LHCP) and linear cases.
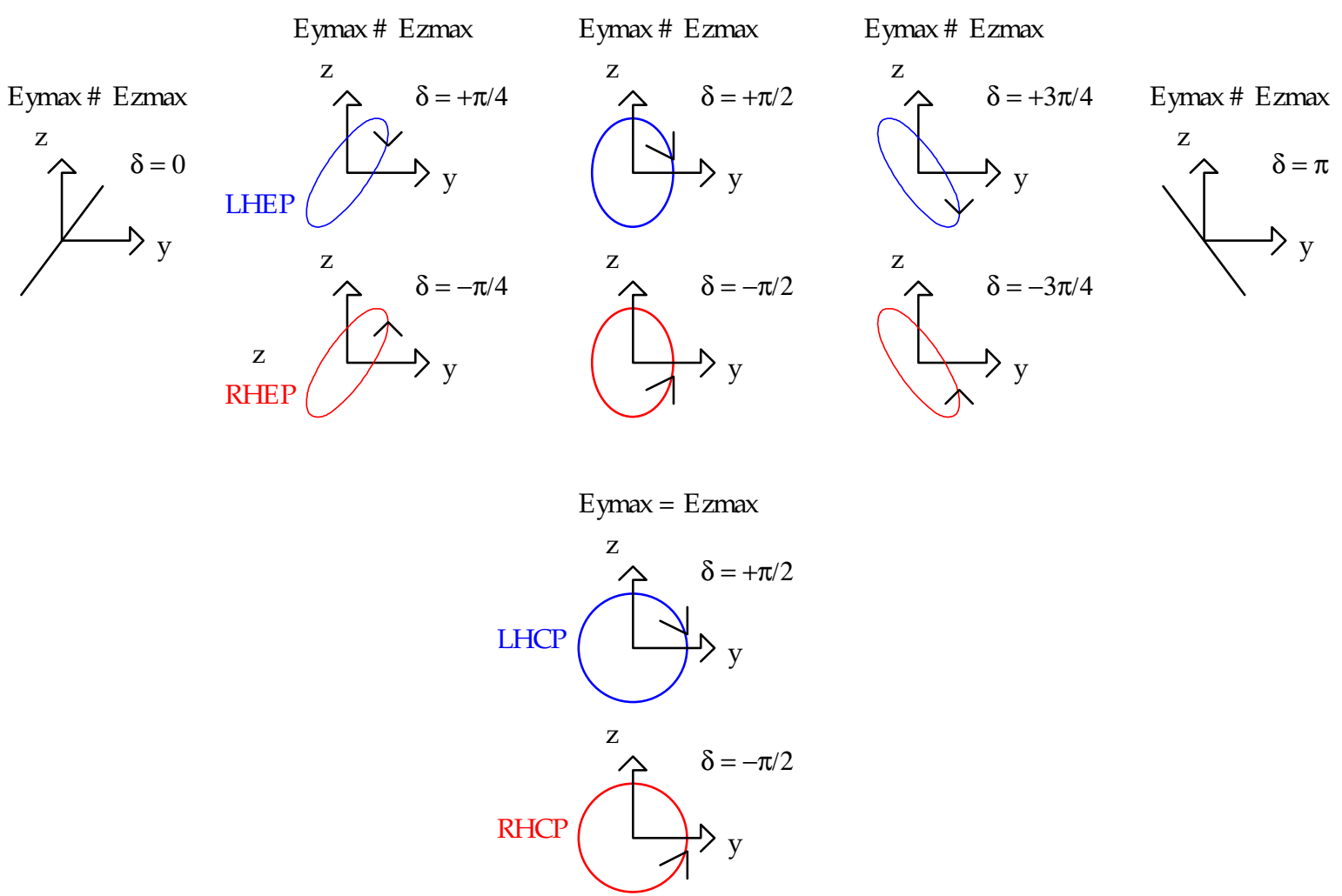

Les us suggest a decomposition of $\mathrm{E}$ into vectors $\mathrm{Y}$ and $\mathrm{Z}$ so that vector $\mathrm{Y}$ belongs to the plane of incidence and vector $\mathrm{Z}$ belongs to the plane of the separating surface between the two media. Note that $\theta 1$ is called the grazing angle (i.e. $90^{\circ}$ - the angle of incidence).

$X=$ direction of incidence

$\mathrm{Y}=$ parallel (or vertical) component

$\mathrm{Z}=$ perpendicular (or horizontal) component

Eymax is denoted $\mathrm{E}_{/ /}$

Ezmax is denoted $\mathrm{E}_{\perp}$

$\mathrm{N}=$ normal of the separating surface

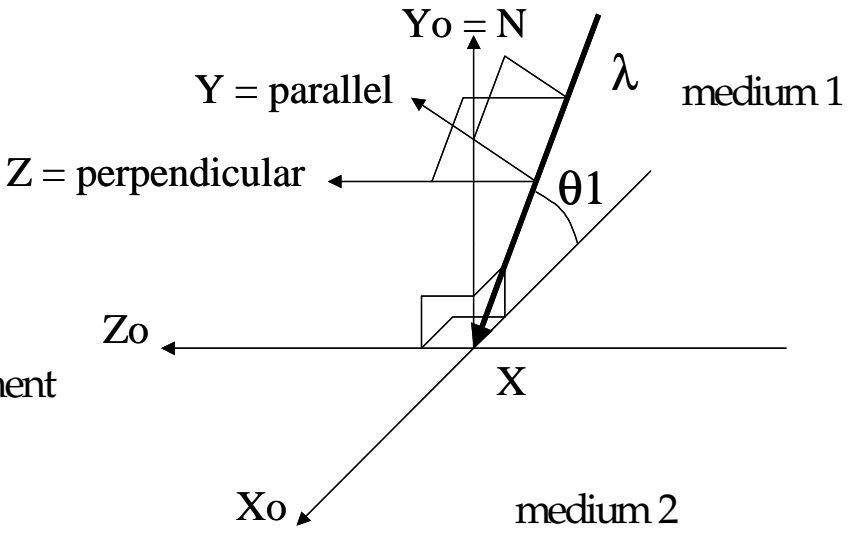


The Snell-Descartes law of propagation enables the directions of incidence, reflection and transmission to be projected in the reference frame $\mathrm{X}_{0} \mathrm{Y}_{0} \mathrm{Z}_{0}$ attached to the separating surface between medium 1 and medium 2 as follows:

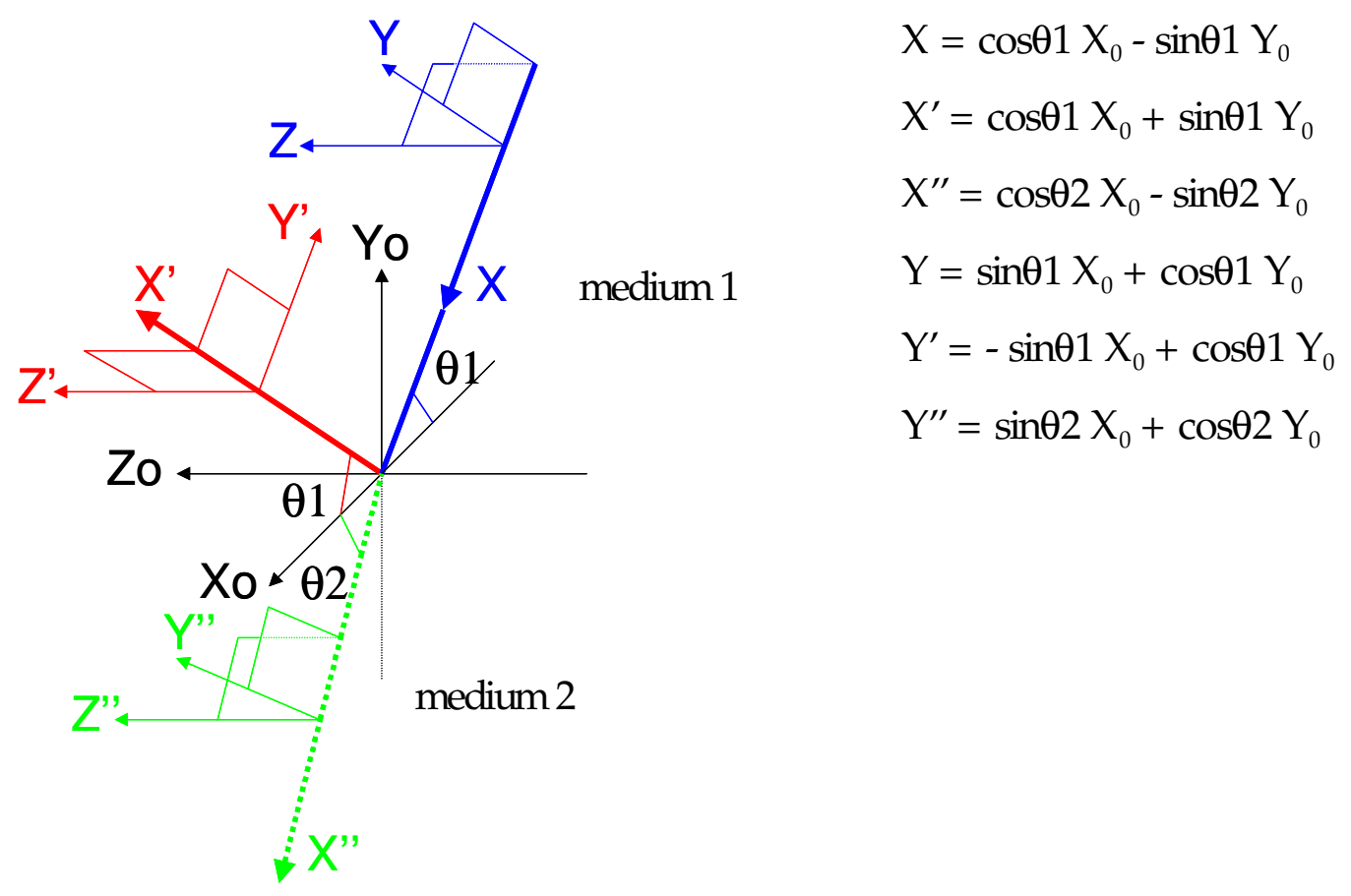

Similarly, the reflected electric field Er have the following decomposition:

$\mathrm{Er}=\mathrm{Ery}+\mathrm{Erz}^{\prime}$

where

$\operatorname{Ery}=R_{/ /} E_{/ /} \sin \left(w t-k x^{\prime}\right) Y^{\prime}$

$E r z^{\prime}=R_{\perp} E_{\perp} \sin \left(w t-k x^{\prime}-\delta\right) Z^{\prime}$

$Y^{\prime}$ and $Z^{\prime}$ are unit vectors that, together with $X^{\prime}$, generate a direct frame $X^{\prime} Y^{\prime} Z^{\prime}$

and $R_{/ /}$and $R_{\perp}$ are respectively the parallel and perpendicular components of the coefficient of reflection, i.e. complex quantities that link both amplitude and phase of the parallel and perpendicular components of the incident and reflected electric fields. 
Lastly, the transmitted electric field Et have the following decomposition:

$\mathrm{Et}=\mathrm{Ety}{ }^{\prime \prime}+\mathrm{Etz}{ }^{\prime \prime}$

where

$E t y "=T_{/ /} E_{/ /} \sin \left(w t-k x^{\prime \prime}\right) Y^{\prime \prime}$

$E t z^{\prime \prime}=T_{\perp} E_{\perp} \sin \left(w t-k x^{\prime \prime}-\delta\right) Z^{\prime \prime}$

$Y^{\prime \prime}$ and $Z^{\prime \prime}$ are unit vectors that, together with $X^{\prime \prime}$, generate a direct frame $X^{\prime \prime} Y^{\prime \prime} Z^{\prime \prime}$

and $T_{/ /}$and $T_{\perp}$ are respectively the parallel and perpendicular components of the coefficient of refraction, i.e. complex quantities that link both amplitude and phase of the parallel and perpendicular components of the incident and transmitted electric fields.

The magnetic field $\mathrm{H}$ can also be decomposed into orthogonal components in exactly the same manner, as well as the reflected and transmitted magnetic fields $\mathrm{Hr}$ and $\mathrm{Ht}$.

Note that the complex impedance links the amplitude and phase of the electric and magnetic fields. Both media have their own complex impedance Z1 and Z2.

The derivation of the coefficient of reflection is based on the expression of the limit conditions on the separating surface between the two media, for both orthogonal components of the electric and magnetic fields. This surface is characterised by $\mathrm{y}_{0}=0$.

For the parallel component of the electric fields, the boundary condition on the separating surface (where $\mathrm{y}_{0}=0$ ) gives:

$$
\begin{aligned}
& \left(\text { Ey }+ \text { Ery }=E^{\prime} y^{\prime \prime}\right)_{\mathrm{y}=0} \\
& \text { Ey }=E_{/ /} \sin \left(w t-k\left(\cos \theta 1 x_{0}-\sin \theta 1 \mathrm{y}_{0}\right)\right)\left(\sin \theta 1 \mathrm{X}_{0}+\cos \theta 1 \mathrm{Y}_{0}\right) \\
& \text { Ery }=\mathrm{R}_{/ /} \mathrm{E}_{/ /} \sin \left(\mathrm{wt}-\mathrm{k}\left(\cos \theta 1 \mathrm{x}_{0}+\sin \theta 1 \mathrm{y}_{0}\right)\right)\left(-\sin \theta 1 \mathrm{X}_{0}+\cos \theta 1 \mathrm{Y}_{0}\right) \\
& \mathrm{Ety}^{\prime \prime}=\mathrm{T}_{/ /} \mathrm{E}_{/ /} \sin \left(\mathrm{wt}-\mathrm{k}\left(\cos \theta 2 \mathrm{x}_{0}-\sin \theta 2 \mathrm{y}_{0}\right)\right)\left(\sin \theta 2 \mathrm{X}_{0}+\cos \theta 2 \mathrm{Y}_{0}\right) \\
& =>\left(1-\mathrm{R}_{/ /}\right) \sin \left(\mathrm{wt}-\mathrm{k}\left(\cos \theta 1 \mathrm{x}_{0}\right)\right)(\sin \theta 1)=\left(\mathrm{T}_{/ /}\right) \sin \left(\mathrm{wt}-\mathrm{k}\left(\cos \theta 2 \mathrm{x}_{0}\right)\right)(\sin \theta 2)
\end{aligned}
$$


The corresponding magnetic fields are in the perpendicular plane:

$\left(\mathrm{Hz}+\mathrm{Hrz}^{\prime}=\mathrm{Htz}^{\prime \prime}\right)_{\mathrm{yo}=0}$

$\mathrm{Hz}=\mathrm{E}_{/ /} / \mathrm{Z} 1 \sin \left(\mathrm{wt}-\mathrm{k}\left(\cos \theta 1 \mathrm{x}_{0}-\sin \theta 1 \mathrm{y}_{0}\right)\right)\left(\mathrm{Z}_{0}\right)$

$\mathrm{Hrz}^{\prime}=\mathrm{R}_{/ /} \mathrm{E}_{/ /} / \mathrm{Z} 1 \sin \left(\mathrm{wt}-\mathrm{k}\left(\cos \theta 1 \mathrm{x}_{0}+\sin \theta 1 \mathrm{y}_{0}\right)\right)\left(\mathrm{Z}_{0}\right)$

$\mathrm{Htz}^{\prime \prime}=\mathrm{T}_{/ /} \mathrm{E}_{/ /} / \mathrm{Z} 2 \sin \left(\mathrm{wt}-\mathrm{k}\left(\cos \theta 2 \mathrm{x}_{0}-\sin \theta 2 \mathrm{y}_{0}\right)\right)\left(\mathrm{Z}_{0}\right)$

$=>\left(\left(1+\mathrm{R}_{/ /}\right) / \mathrm{Z} 1\right) \sin \left(\mathrm{wt}-\mathrm{k}\left(\cos \theta 1 \mathrm{x}_{0}\right)\right)=\left(\mathrm{T}_{/ /} / \mathrm{Z} 2\right) \sin \left(\mathrm{wt}-\mathrm{k}\left(\cos \theta 2 \mathrm{x}_{0}\right)\right)$

So: $\left(1+\mathrm{R}_{/ /}\right) /\left(1-\mathrm{R}_{/ /}\right)=(\mathrm{Z} 1 \sin \theta 1) /(\mathrm{Z} 2 \sin \theta 2)$

For the perpendicular component of the electric fields, the boundary condition on the separating surface (where $\left.y_{0}=0\right)$ gives:

$\left(\mathrm{Ez}+\mathrm{Erz}^{\prime}=\mathrm{Etz}\right)_{\mathrm{yo}=0}$

$\mathrm{Ez}=\mathrm{E}_{\perp} \sin \left(\mathrm{wt}-\mathrm{k}\left(\cos \theta 1 \mathrm{x}_{0}-\sin \theta 1 \mathrm{y}_{0}\right)-\delta\right)\left(\mathrm{Z}_{0}\right)$

$E r z^{\prime}=R_{\perp} E_{\perp} \sin \left(w t-k\left(\cos \theta 1 x_{0}+\sin \theta 1 y_{0}\right)-\delta\right)\left(Z_{0}\right)$

$\mathrm{Etz}^{\prime \prime}=\mathrm{T}_{\perp} \mathrm{E}_{\perp} \sin \left(\mathrm{wt}-\mathrm{k}\left(\cos \theta 2 \mathrm{x}_{0}-\sin \theta 2 \mathrm{y}_{0}\right)-\delta\right)\left(\mathrm{Z}_{0}\right)$

$=>\left(1+R_{\perp}\right) \sin \left(u t-k\left(\cos \theta 1 x_{0}\right)\right)=\left(T_{\perp}\right) \sin \left(w t-k\left(\cos \theta 2 x_{0}\right)\right)$

The corresponding magnetic fields are in the parallel plane:

$\left(\mathrm{Hy}+\text { Hry }^{\prime}=\text { Hty }^{\prime \prime}\right)_{\mathrm{yo}=0}$

$\mathrm{Hy}=\mathrm{E}_{\perp} / \mathrm{Z1} \sin \left(\mathrm{wt}-\mathrm{k}\left(\cos \theta 1 \mathrm{x}_{0}-\sin \theta 1 \mathrm{y}_{0}\right)\right)\left(-\sin \theta 1 \mathrm{X}_{0}-\cos \theta 1 \mathrm{Y}_{0}\right)$

Hry $=R_{\perp} E_{\perp} / Z 1 \sin \left(w t-k\left(\cos \theta 1 x_{0}+\sin \theta 1 y_{0}\right)\right)\left(\sin \theta 1 X_{0}-\cos \theta 1 Y_{0}\right)$

$\mathrm{Hty}^{\prime \prime}=\mathrm{T}_{\perp} \mathrm{E}_{\perp} / \mathrm{Z} 2 \sin \left(\right.$ ut $\left.-\mathrm{k}\left(\cos \theta 2 \mathrm{x}_{0}-\sin \theta 2 \mathrm{y}_{0}\right)\right)\left(-\sin \theta 2 \mathrm{X}_{0}-\cos \theta 2 \mathrm{Y}_{0}\right)$

$=>\left(\left(1-\mathrm{R}_{\perp}\right) / \mathrm{Z} 1\right) \sin \left(\mathrm{ht}-\mathrm{k}\left(\cos \theta 1 \mathrm{x}_{0}\right)\right)(-\sin \theta 1)=\left(\mathrm{T}_{\perp} / \mathrm{Z} 2\right) \sin \left(\mathrm{ht}-\mathrm{k}\left(\cos \theta 2 \mathrm{x}_{0}\right)\right)(-\sin \theta 2)$

So: $\left(1+R_{\perp}\right) /\left(1-R_{\perp}\right)=(Z 2 \sin \theta 1) /(Z 1 \sin \theta 2)$ 
Replacing Z1 for air (considered as free space) and Z2 for a given material by their values:

$\mathrm{Z} 1=\sqrt{\frac{\mu_{0}}{\varepsilon_{0}}}$ and $\mathrm{Z} 2=\sqrt{\frac{\mu_{0} \mu_{r}}{\varepsilon_{0} \varepsilon}}$

$\mu_{0}=4 \pi 10^{-7} \mathrm{H} \mathrm{m}^{-1}$ (vacuum permeability)

$\varepsilon_{0}=(1 / 36 \pi) 10^{-9} \mathrm{~F} \mathrm{~m}^{-1}$ (vacuum permittivity)

where $\varepsilon$ is the complex dielectric constant of the material:

$\varepsilon=\varepsilon_{\mathrm{r}}-\mathrm{j} \sigma / \omega \varepsilon_{0}$

and where

$\omega$ is the pulsation of the carrier wave $\left(2 \pi * 1.5754210^{9} \mathrm{rad} \mathrm{s}^{-1}\right.$ for L1 GPS frequency) and $\sigma$ is the conductivity of the material;

and using the law of the refraction (with the permeability of the material $\mu_{\mathrm{r}}$ supposed $\sim 1$ ):

$\sqrt{\mu_{0} \varepsilon_{0}} \cos \theta 1=\sqrt{\mu_{0} \mu_{\mathrm{r}} \varepsilon_{0} \varepsilon} \cos \theta 2$

the parallel coefficient of reflection is found to be:

$$
\mathrm{R}_{/ /}=\frac{\varepsilon \sin \theta 1-\sqrt{\varepsilon-\cos ^{2} \theta} 1}{\varepsilon \sin \theta 1+\sqrt{\varepsilon-\cos ^{2} \theta 1}}
$$

and the perpendicular coefficient of reflection:

$$
\mathrm{R}_{\perp}=\frac{\sin \theta 1-\sqrt{\varepsilon-\cos ^{2} \theta 1}}{\sin \theta 1+\sqrt{\varepsilon-\cos ^{2} \theta 1}}
$$


The magnitude and argument of the complex coefficients of reflection $R_{/ /}$and $R_{\perp}$ give respectively the attenuation and the phase shift of the parallel and perpendicular components of the electric field when this is reflected.

Let us examine these two components on two examples: water (running water) and metal (steel). The order of magnitude of their conductivity and permittivity are:

$\sigma \sim 0.2 \mathrm{~S} \mathrm{~m}^{-1}$ and $\varepsilon_{\mathrm{r}} \sim 80$ (running water)

$\sigma \sim 7.710^{6} \mathrm{~S} \mathrm{~m}^{-1}$ and $\varepsilon_{\mathrm{r}}<<\sigma / \omega \varepsilon_{0}$ (steel)

Note: for a conductive material, the complex dielectric constant tends to be a pure imaginary.

The following figures give the magnitude and argument of both components of the coefficient of reflection. The parallel component equals zero at certain grazing angle, named the Brewster angle $\theta b$.

If the incident electric field is RHCP (like with GPS), the reflected electric field is:

- RHEP for $\theta 1<\theta b$ (there is no inversion of the sign of the polarisation, because both parallel and perpendicular components are $180^{\circ}$ shifted);

- the polarisation is linear at $\theta b$;

- LHEP for $\theta 1>\theta b$ (there is an inversion of the sign of the polarisation, because the parallel component is not shifted whereas that perpendicular is $180^{\circ}$ shifted).

The polarisation is circular when both components of the coefficient of reflection have the same magnitude, which is always the case for a reflection on metal. 

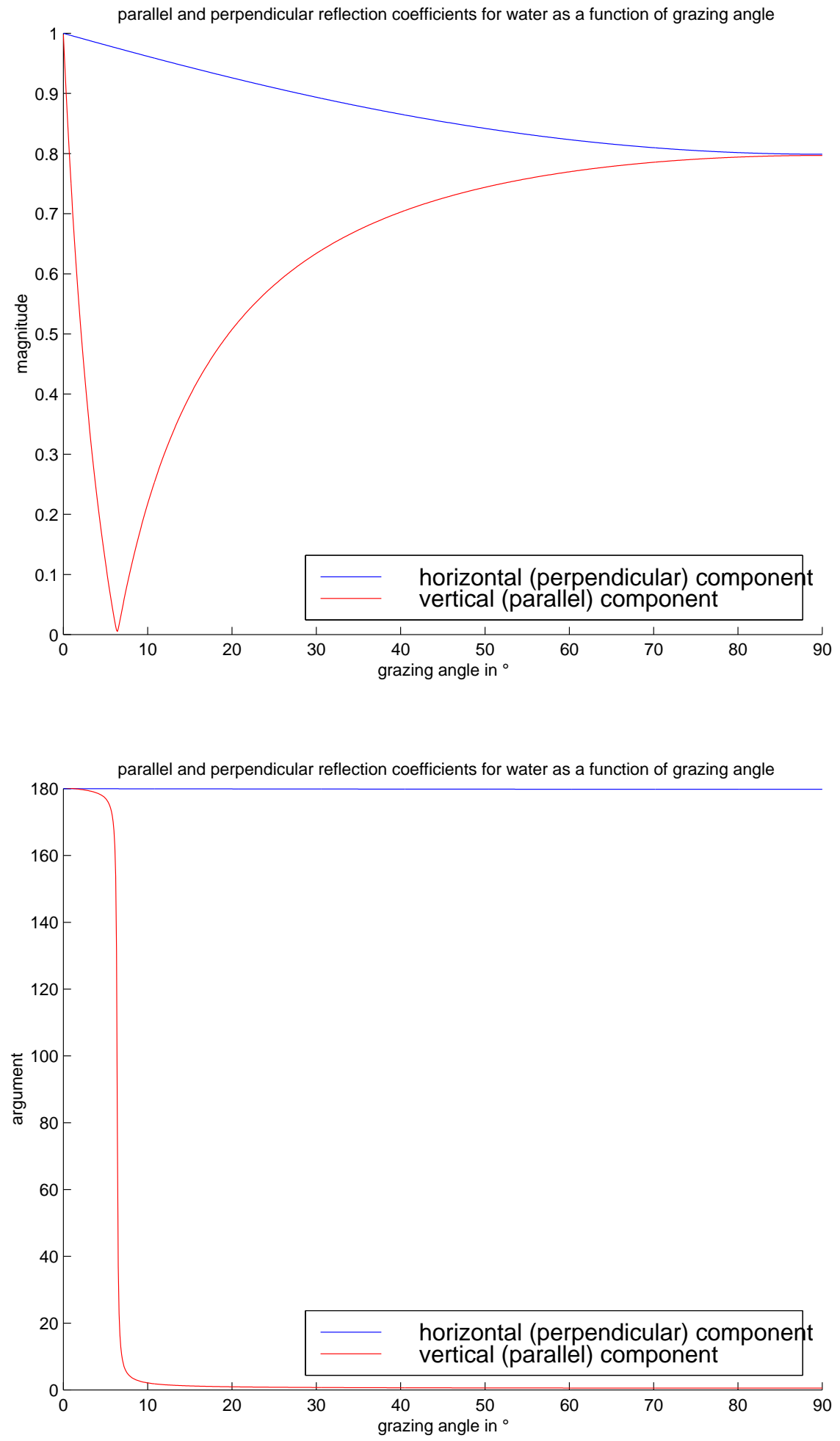

Figure A2.1: parallel and perpendicular reflection coefficients typical of water at hyperfrequencies. 

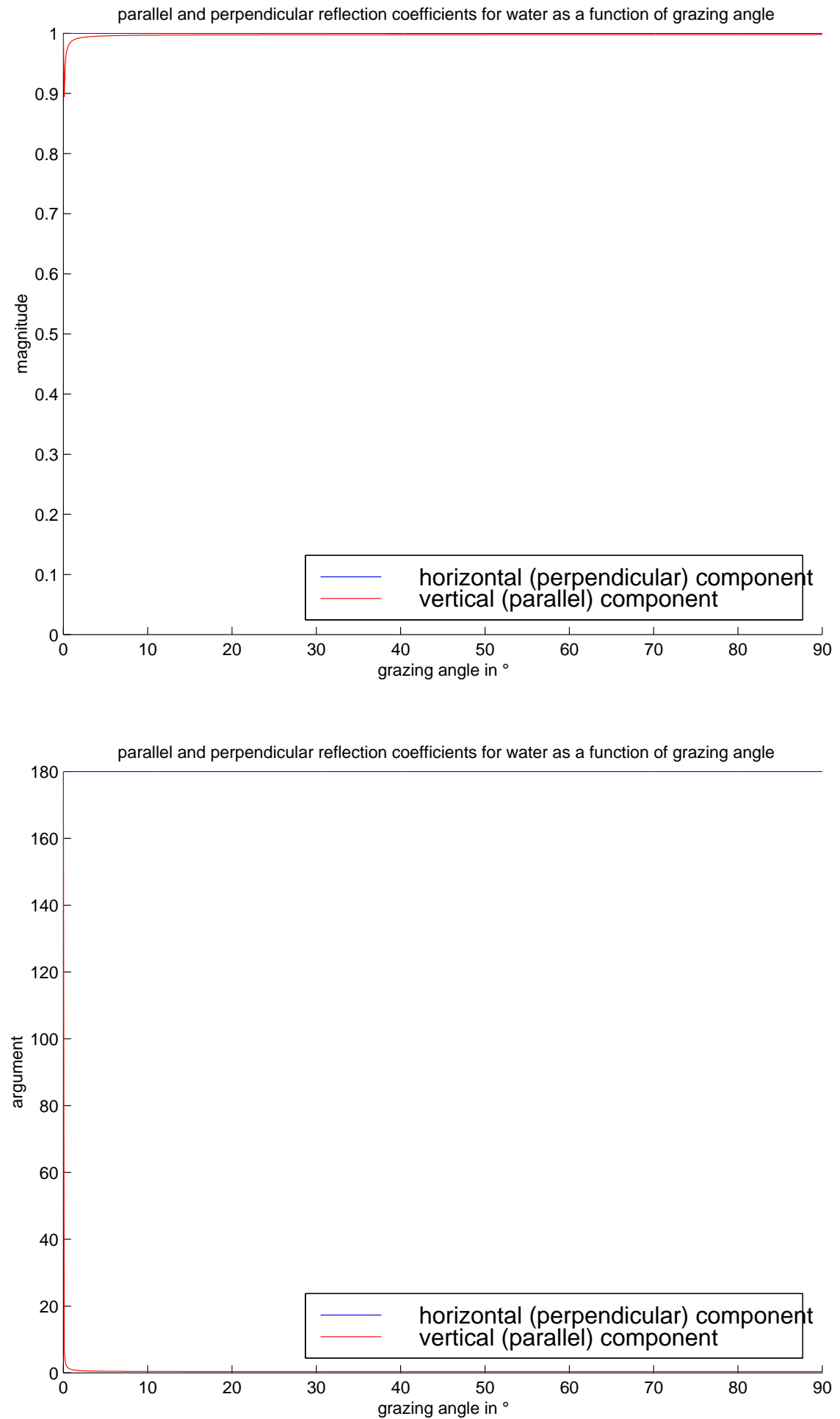

Figure A2.2: parallel and perpendicular reflection coefficients typical of metal at hyperfrequencies. 
APPENDIX 3: AERIAL PHOTO OF THE SESSYL SITE

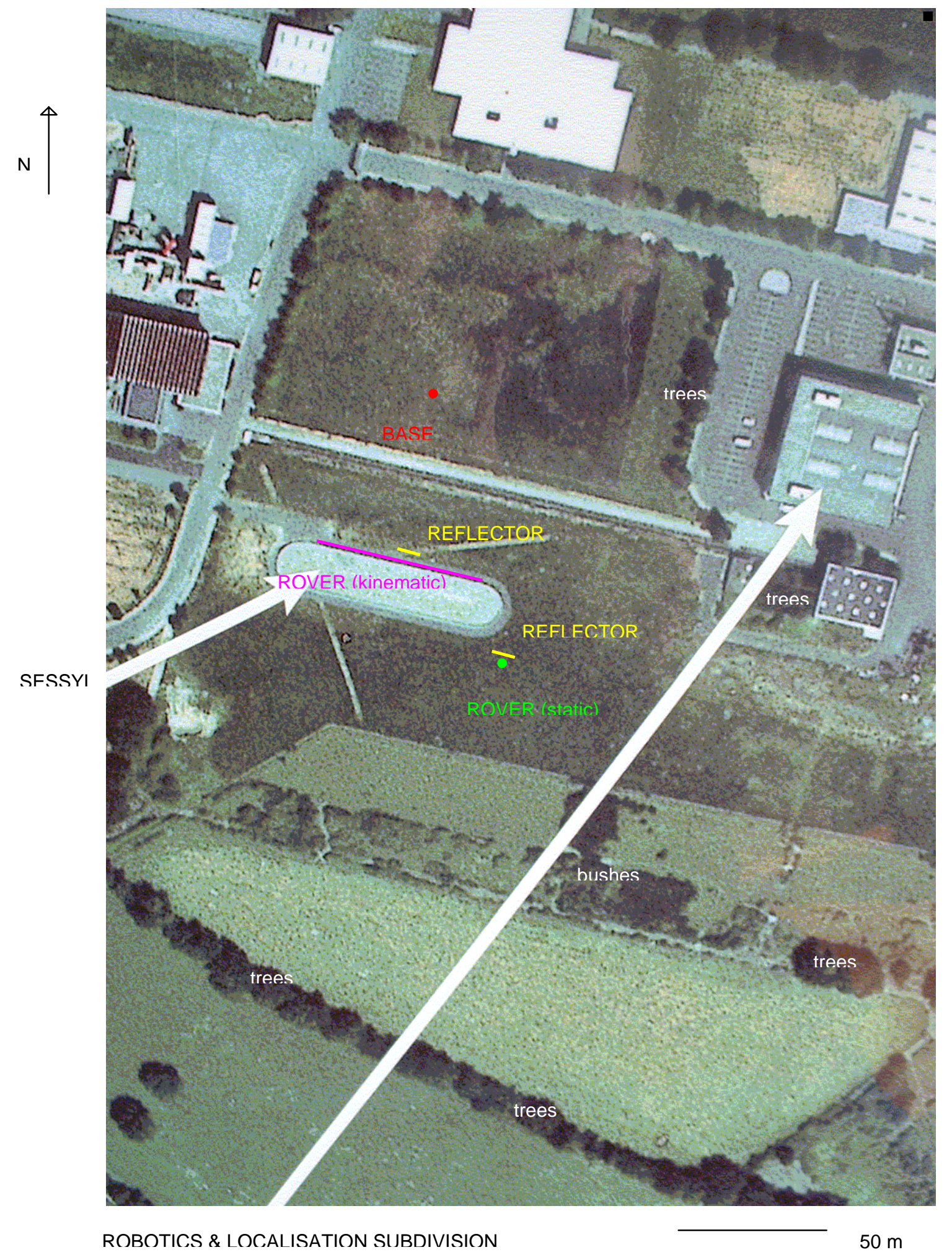




\section{APPENDIX 4: RESULTS OF THE STATIC TESTS WITH CHOKE-RING}

\section{ANTENNAS}

During the preliminary campaign of tests in 2002, both lightweight and choke-ring antennas were tested. Appendix 4 summarises the results of the static tests performed with choke-ring, those with lightweight being presented in Chapter 6 .

The O-C DD of L1 phase resulting from the tests with the choke-ring antennas are given in Fig. A4.1a. The satellite SV2 has been selected. Multipath is visible and the frequency, phase and amplitude of the resulting phase error correspond closely to those predicted by multipath modelling. Note that the amplitude is significantly reduced compared with that for the lightweight antennas.

The periods of multipath occurrence (determined geometrically with the help of the precise reference positioning) are identified by a "green window" superimposed onto the time series.

It can be seen from Fig. A4.1b that when a choke-ring antenna is used and corrections to the L1 phase measurements made using the phase MMW, it appears that the standard deviation of the phase error, despite multipath, equals that when no multipath exists.

The same test was repeated with the reflector placed at a distance of about $2 \mathrm{~m}$ from the antenna. The resulting time series are shown in Fig. A4.2a. At this distance, the additional path length corresponding to the satellite observed here is between $2 \mathrm{~m}$ and $4 \mathrm{~m}$ (whereas it was between $6.5 \mathrm{~m}$ and $8.5 \mathrm{~m}$ with the reflector at $5 \mathrm{~m}$ ).

As expected for this case, the phase MMW correlator is much less effective in mitigating the multipath error, due to the fact the additional path length is well under the $7.5 \mathrm{~m}$ threshold. 
The results with the panel at $5 \mathrm{~m}$ are summarised below (for SV2 only).

\begin{tabular}{cccc}
\hline Choke-ring antenna - SV2 & Std dev (PMMW off) & Std dev (PMMW on) & Gain \\
\hline reflector $5 \mathrm{~m}$ & $2.5 \mathrm{~mm}$ & $2.0 \mathrm{~mm}$ & $20 \%$ \\
no reflector & $2.3 \mathrm{~mm}$ & $2.3 \mathrm{~mm}$ & 0 \\
\hline
\end{tabular}
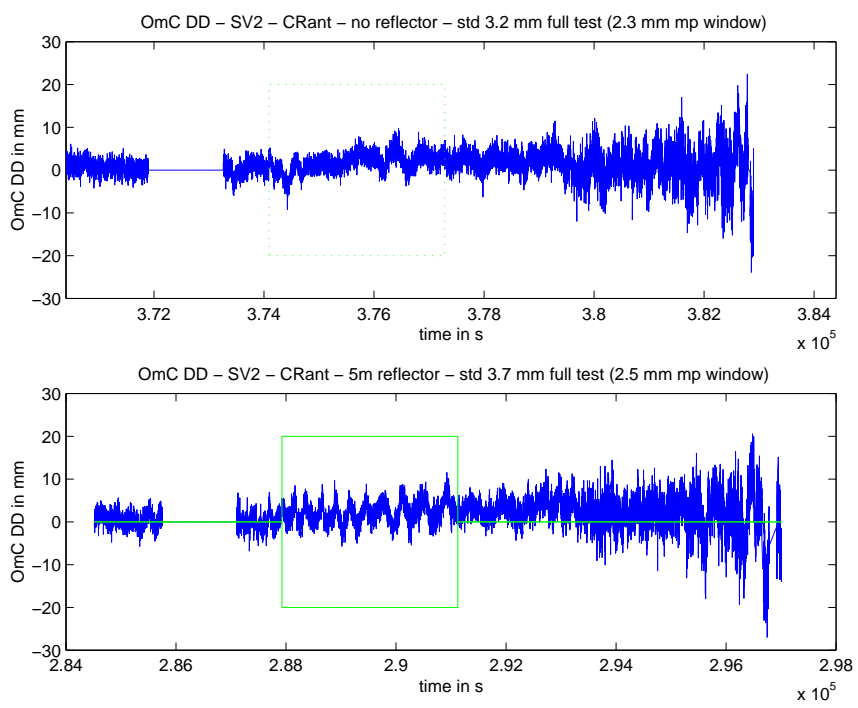

Figure A4.1a: static choke-ring antenna (at $5 \mathrm{~m}$ ), no correction
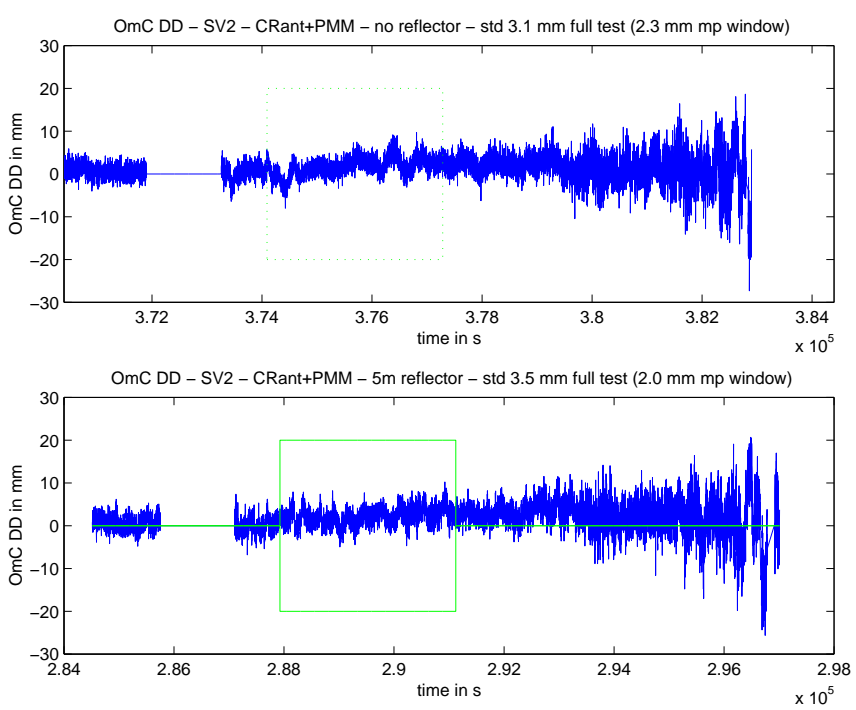

Figure A4.1b: static choke-ring antenna (at $5 \mathrm{~m}$ ), phase MMW correction applied 
The results with the panel at $2 \mathrm{~m}$ are summarised below (for SV2 only).

\begin{tabular}{cccc}
\hline Choke-ring antenna - SV2 & Std dev (PMMW off) & Std dev (PMMW on) & Gain \\
\hline reflector $2 \mathrm{~m}$ & $2.7 \mathrm{~mm}$ & $2.5 \mathrm{~mm}$ & $7 \%$ \\
no reflector & $2.3 \mathrm{~mm}$ & $2.3 \mathrm{~mm}$ & 0 \\
\hline
\end{tabular}
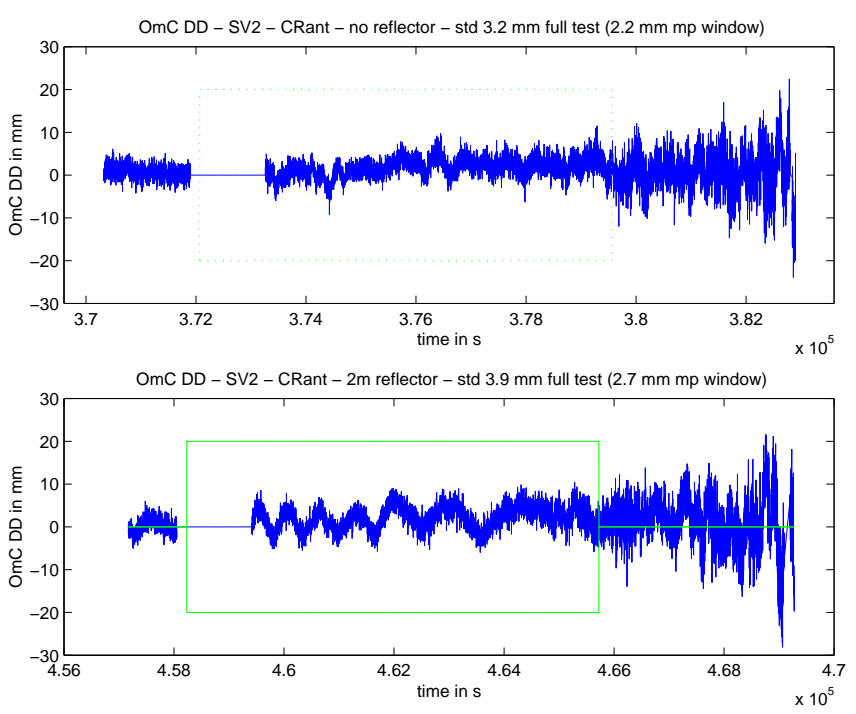

Figure A4.2a: static choke-ring antenna (at $2 \mathrm{~m}$ ), no correction
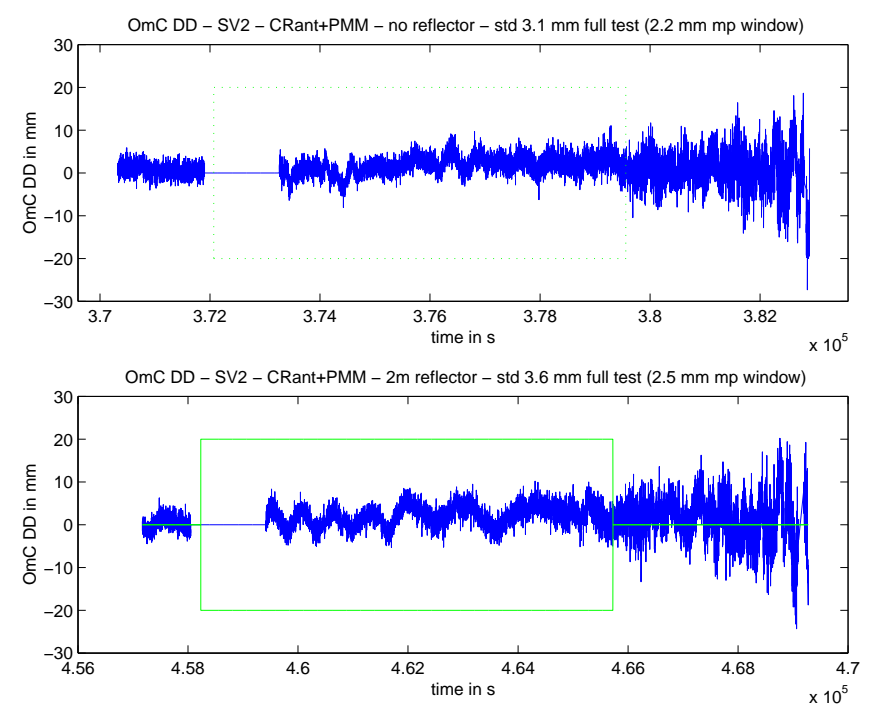

Figure A4.2b: static choke-ring antenna (at $2 \mathrm{~m}$ ), phase MMW correction applied 
For memory, the same time series and statistical results are given for the same satellite in the case of using lightweight antennas, and for the reflector placed at a distance of $5 \mathrm{~m}$. See and compare Fig. A4.3a and A4.3b to respectively Fig. A4.1a and A4.1b: it is obvious that the interest of the phase MMW is greater with lightweight than with choke-ring.

\begin{tabular}{cccc}
\hline Lightweight antenna - SV2 & Std dev (PMMW off) & Std dev (PMMW on) & Gain \\
\hline reflector $5 \mathrm{~m}$ & $3.8 \mathrm{~mm}$ & $2.5 \mathrm{~mm}$ & $34 \%$ \\
no reflector & $2.2 \mathrm{~mm}$ & $1.9 \mathrm{~mm}$ & $14 \%$ \\
\hline
\end{tabular}
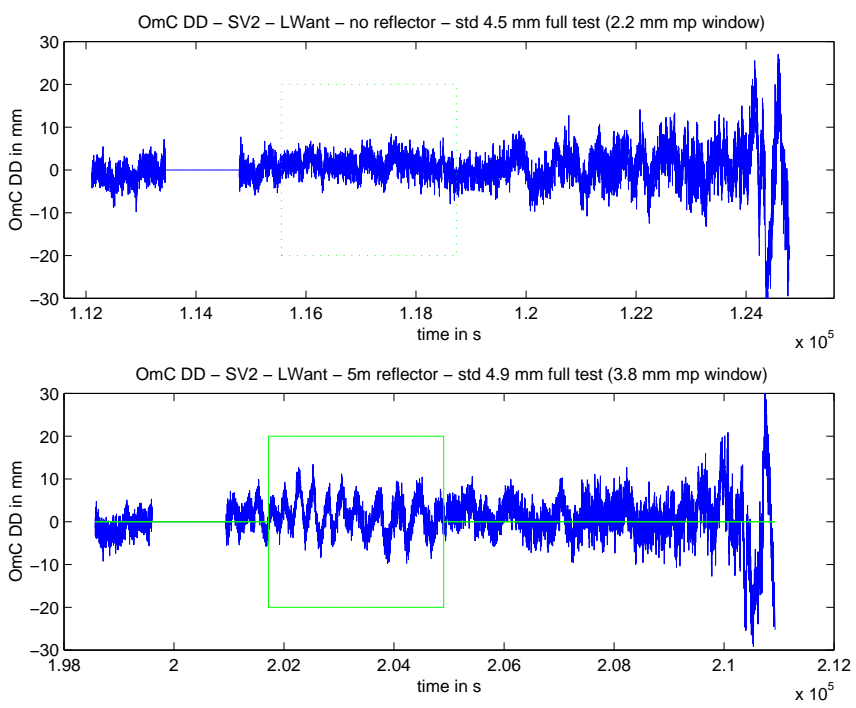

Figure A4.3a: static lightweight antenna (at $5 \mathrm{~m}$ ), no correction
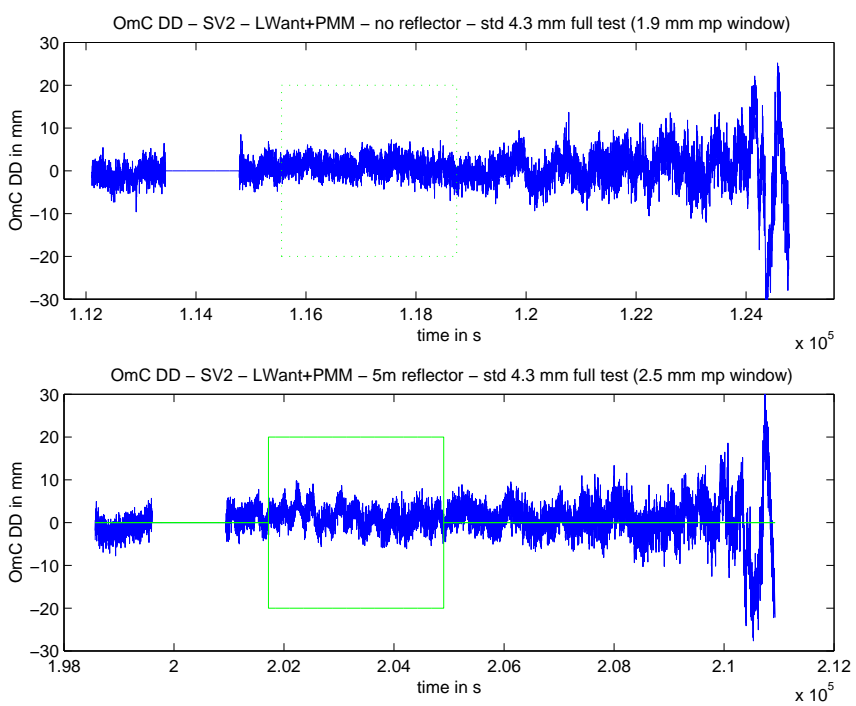

Figure A4.3b: static lightweight antenna (at $5 \mathrm{~m}$ ), phase MMW correction applied 


\section{APPENDIX 5: STUDY OF THE BACKGROUND MULTIPATH FROMSESSYL}

The preliminary campaign of tests that was done in 2002 showed SESSYL originating multipath. This appendix demonstrates how it was identified and modelled.

During these tests, the base station was set in the grass field surrounding the SESSYL test bed. The rover antenna was mounted on the SESSYL platform as shown on the Fig. A5.1. Its co-ordinates are known in 3 dimensions with an accuracy of $1 \mathrm{~mm}(1 \sigma)$ when it is moving at a speed of $0.05 \mathrm{~m} / \mathrm{s}$. The baseline length never exceeded $100 \mathrm{~m}$. The SESSYL reference data were time tagged by means of a PPS acquisition, for synchronous comparison with the GPS data logged by the unit being tested.

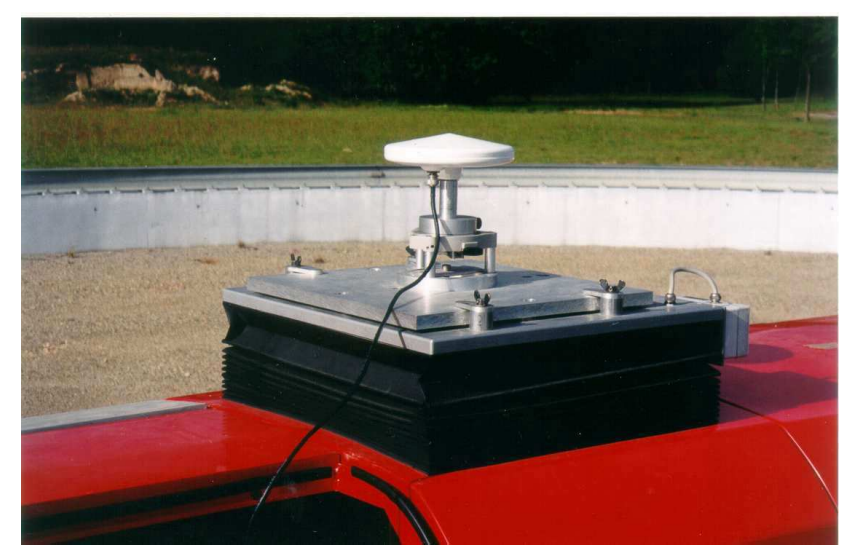

Figure A5.1: the lightweight antenna on the SESSYL platform

Kinematic tests have typically been carried out with SESSYL repeatedly passing in front of the panel at low speed $(0.05 \mathrm{~m} / \mathrm{s})$ on the first of the straight sections of the track, and then returning at high speed back to the starting point. During the preliminary tests, the complete cycle that SESSYL performed repeats once every 40 minutes, and the carriage is in front of the panel for around $100 \mathrm{~s}$ (the $5 \mathrm{~m}$ long panel was placed along the track, near the middle of the $50 \mathrm{~m}$ first straight). 
GPS data are collected continuously whereas the SESSYL data are limited to the low speed first section of the cycle. On these sections, where both sets of data are registered, the observed double differences of phase can be compared to that computed theoretically with the known positions of SESSYL in time.

Initial analysis of the GPS data collected during the preliminary tests indicated long period multipath effects, and this appendix contains details of the analysis of these. Note that this effect is important because it leads to systematic offsets in the observed multipath for the short time periods during which reflections are received from the panel.

Since the antenna does not rotate during the test, the reference frame $\mathrm{RO}$ (obtained by translating the SESSYL local reference frame RL onto the L1 phase centre of the rover antenna, see Chapter $6, \S 6.2 .1)$ can still be used as the antenna body-fixed frame. Its origin changes in time, but not its orientation. The SESSYL platform is fixed in the reference frame $\mathrm{RO}$. The exact co-ordinates of the edges of the platform in $\mathrm{RO}$ permit a theoretical computation of the multipath window time zone and phase error (see again Chapter 6).

The same geometrical equations that were used in the computation of the reflection on the panel were used to compute a multipath window corresponding to the SESSYL platform. Note that this window is considerably longer in time, because the distance to the reflector is much closer, and also the multipath concerns all the satellites irrespective of their azimuth (see Fig. A5.2). The panel creates multipath only for satellites that are in front of it. 


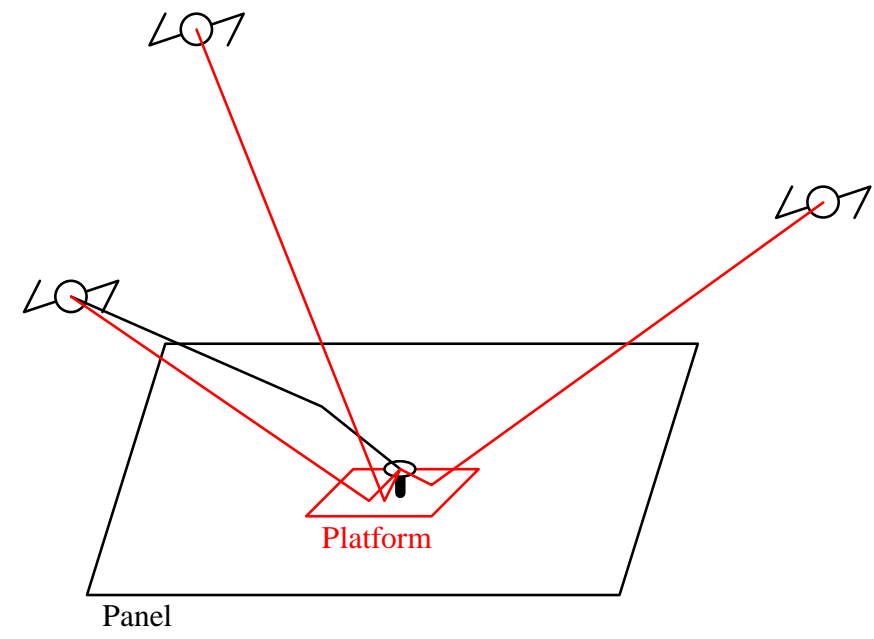

Figure A5.2: 3D scheme of the environment of the antenna

Contrary to the situation of the panel, the SESSYL platform is prone to create multipath for the higher satellite, that chosen for differencing. Hence, in computing the model of multipath error for DD, the contributions from both satellites must be computed and combined.

Hence, Fig. A5.3 shows the multipath error for the successive differencing satellites. Note that the multipath phase error shows no discontinuity when changing the differencing satellite, because when changing, two consecutive differencing satellites have the same elevation.

This figure displays successively:

- $\quad$ the multipath phase error (in "red") due to the SESSYL platform on SV2 phase data. The "green window" delimits the time zone when the SESSYL platform effectively causes multipath for this satellite. The "red line" extends beyond the "green window" since it is computed assuming an infinite sized reflector.

- $\quad$ the multipath phase error (in "black") due to the SESSYL platform on the differencing satellites (SV22, SV2, SV3 and SV31) phase data. There is no delimiting window since 
the differencing satellites are always affected, due to their high elevation. The vertical "black lines" show the epochs when the differencing satellite was changed.

- the difference of the two preceding multipath phase error time series (in "magenta"), which corresponds to the modelled bias observed on the double differences. Outside the "green window", the only remaining multipath phase error is that related to the differencing satellites Note that no multipath phase error is computed for the base station, which is set up in a clean environment.
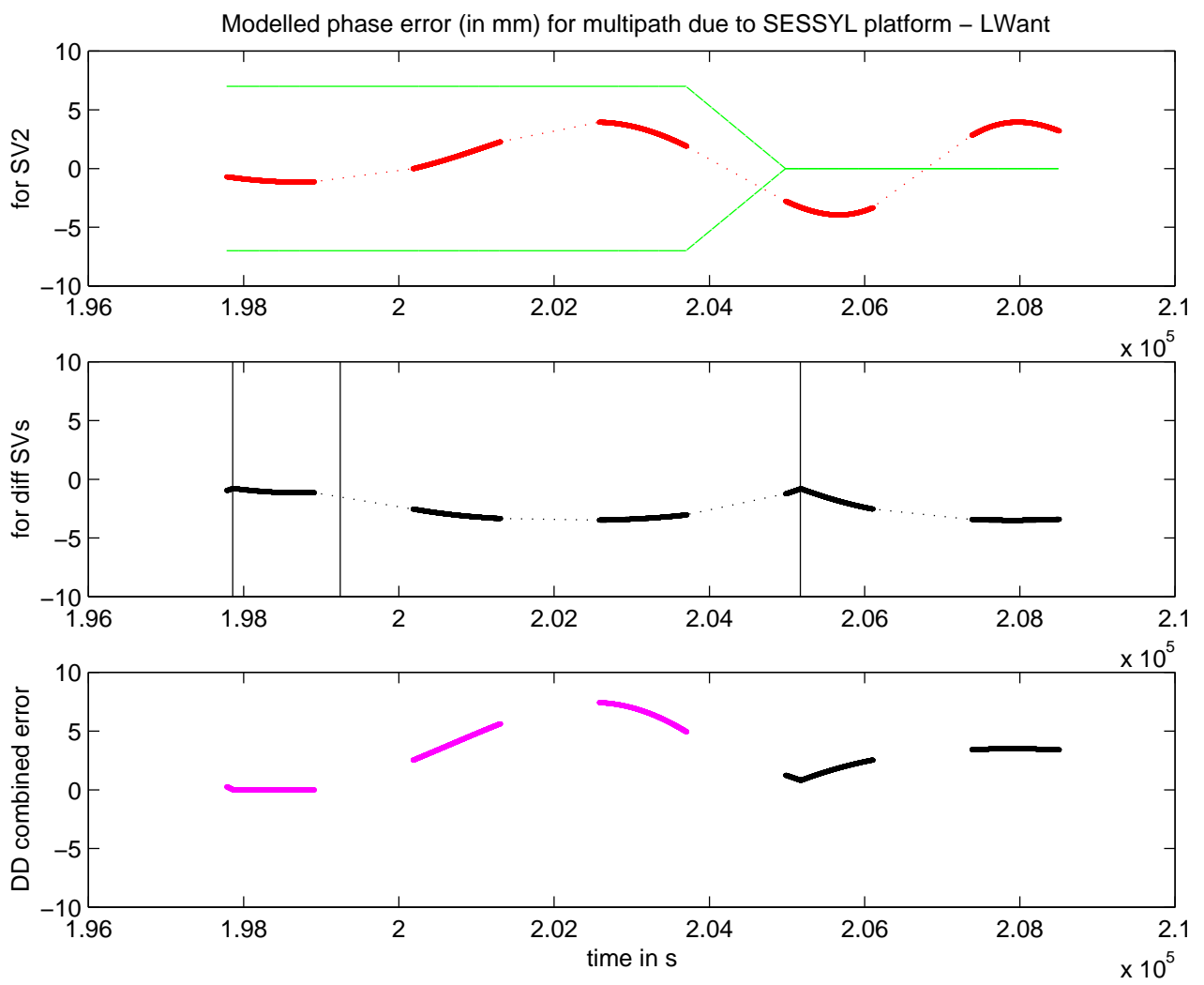

Figure A5.3: modelled multipath errors combined in DD 
The O-C DD of L1 phase are given in Fig. A5.4 to A5.6 for respectively the lightweight (LW) antenna at $5 \mathrm{~m}$, the choke-ring $(\mathrm{CR})$ antenna at $5 \mathrm{~m}$, and the $\mathrm{LW}$ antenna at $2 \mathrm{~m}$. The satellite SV2 was selected.

By visual inspection in Fig. A5.4 and A5.6, it can be seen that the long period multipath phase error is partially modelled. Here, the value of the ratio of amplitude of the direct and reflected signals $\alpha$ has not been modified in function of the elevation and azimuth. So $\alpha$ is fixed in these figures, and it comes from the static preliminary test with the panel (see Chapter 6, table 6.2), where the angles of the signals (neither direct nor reflected) were not the same as in the SESSYL platform situation. Consequently the amplitude of the predicted error may not agree exactly with that observed.

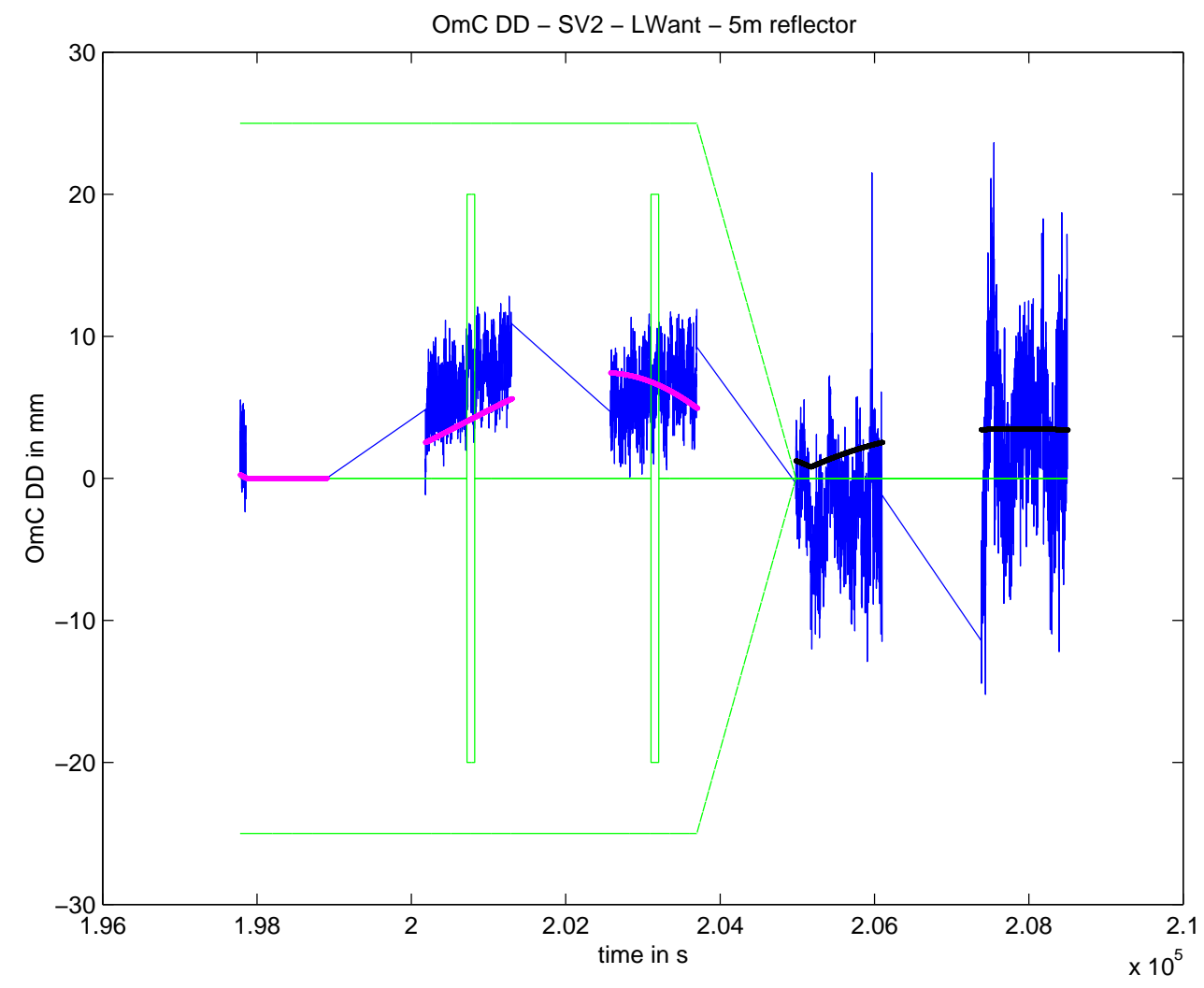

Figure A5.4: O-C DD of L1 phase for the LW test at $5 \mathrm{~m}$ 
This long period multipath error is almost suppressed when using a choke-ring antenna, as shown in Fig. A5.5.

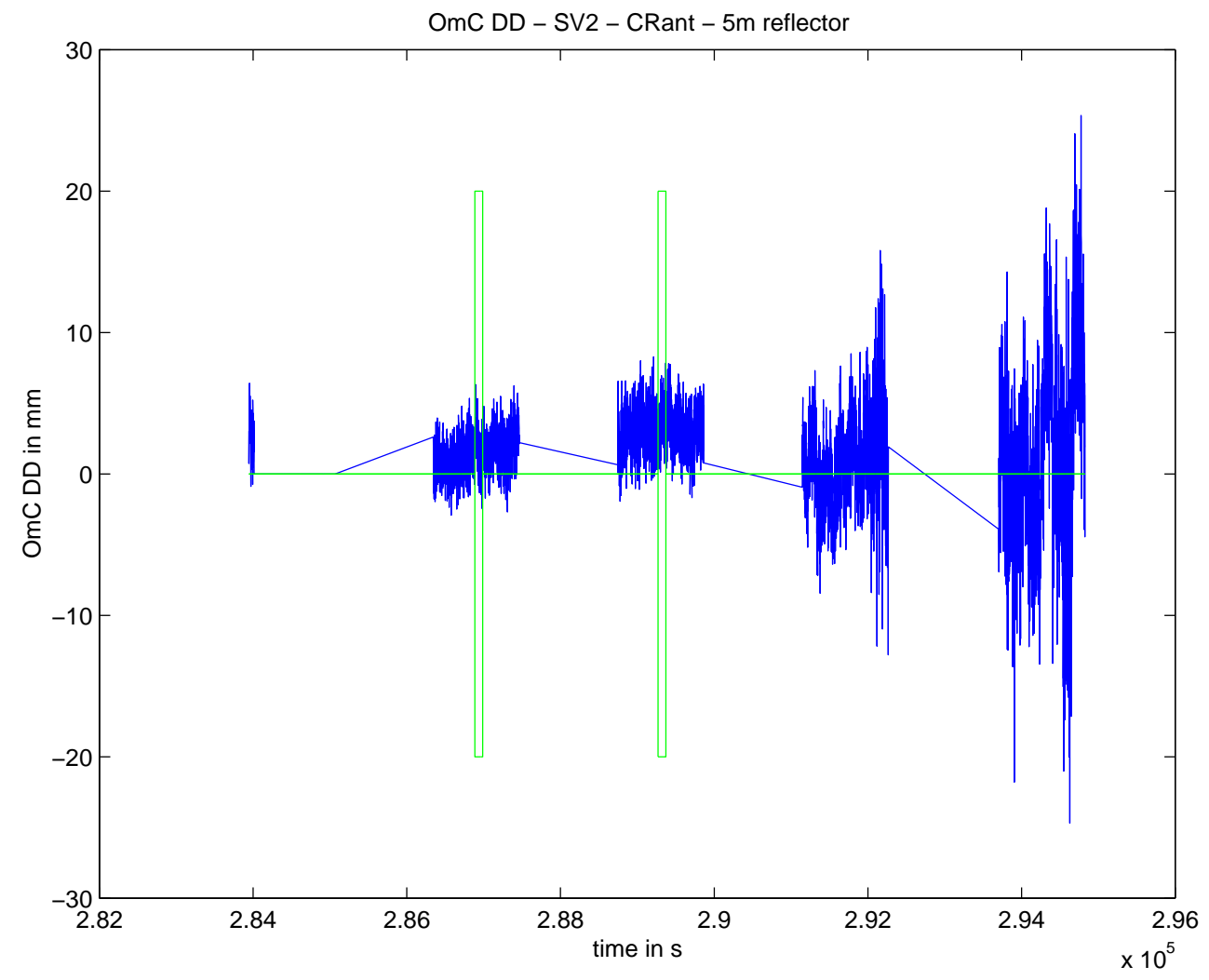

Figure A5.5: O-C DD of L1 phase for the CR test at $5 \mathrm{~m}$

Lastly, as expected, this long period multipath error does not depend on the location of the panel (confirming it is only due to SESSYL). The same trend remains either at $5 \mathrm{~m}$ or at $2 \mathrm{~m}$ tests (see Fig. A5.4 and A5.6). At $2 \mathrm{~m}$, compared to $5 \mathrm{~m}$, note that there is an additional "green window" corresponding to multipath due to the panel (multipath occurs more often with a closer reflector). 


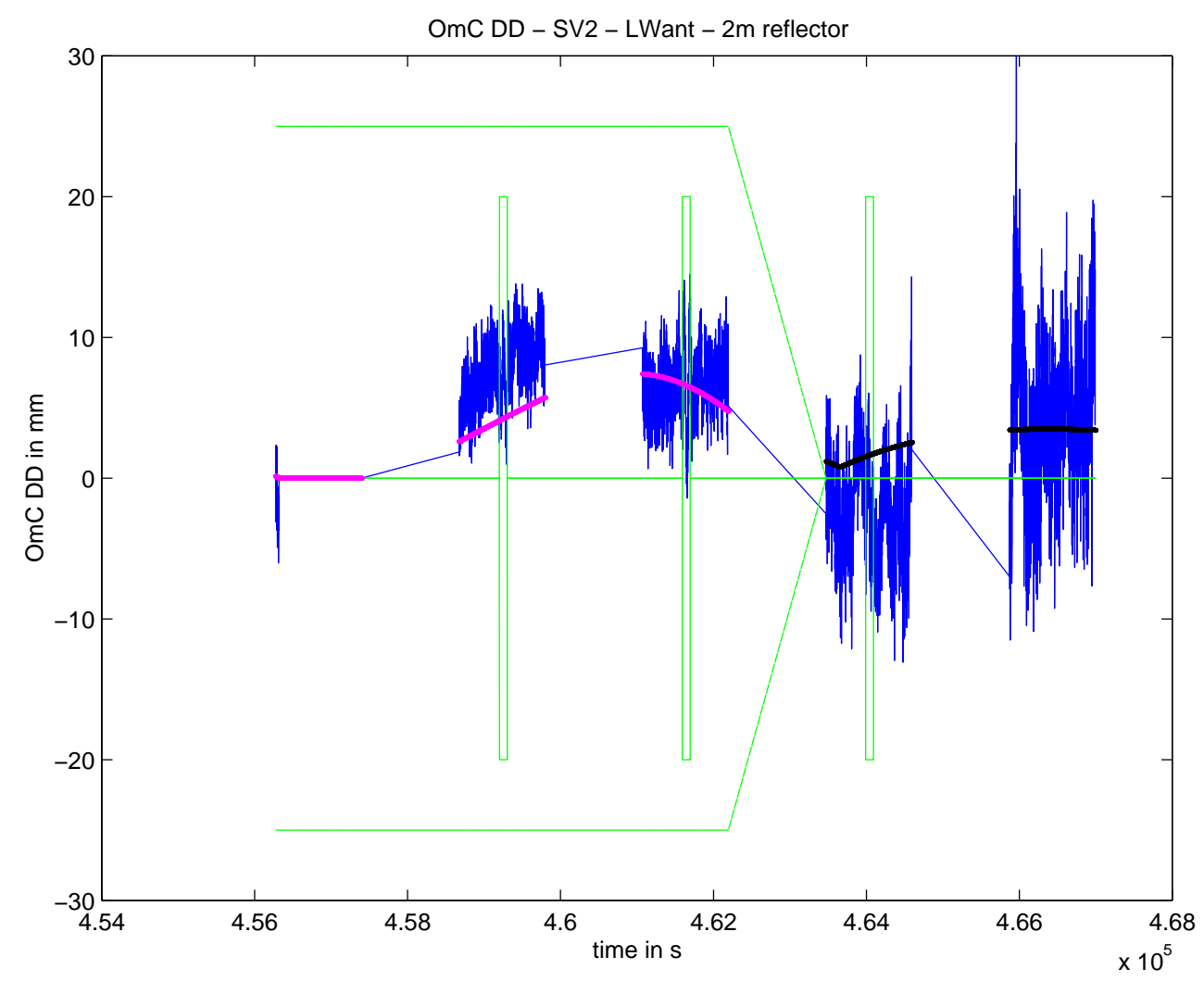

Figure A5.6: O-C DD of L1 phase for the LW test at $2 \mathrm{~m}$

In conclusion, one can state that the measurements are biased due to the multipath caused by the metallic plate (i.e. the SESSYL platform) on top of which the antenna is placed. This bias needs to be taken into account if it is required to analyse the multipath due to the panel only. This SESSYL originating multipath could be considered globally as a drawback of the SESSYL test bed. But in the frame of this research, the SESSYL platform can be considered as a secondary source of multipath (in addition to the panel), and the effectiveness of any mitigation technique being tested will be seen for both sources. So, it here appears that the phase MMW technique actually fails in mitigating multipath due to such a close reflector. 